\title{
MEASUREMENT OF THE CHARGED KAON MASS WITH THE MIPP $\mathrm{RICH}$
}

Nicholas J. Graf

Submitted to the faculty of the University Graduate School

in partial fulfillment of the requirements

for the degree

Doctor of Philosophy

in the Department of Physics,

Indiana University

August 2008 
Accepted by the Graduate Faculty, Indiana University, in partial fulfillment of the requirements for the degree of Doctor of Philosophy.

Doctoral Committee

Mark Messier, Ph.D.

Brian Serot

Jon Urheim

Rick Van Kooten

Date of Oral Examination

July 25, 2008 


\section{Acknowledgments}

Without the help and support of many people, I would not have been able to complete my doctoral work. I am especially grateful to my family. My parents provided a great deal of support during my educational career and always encouraged me to pursue my interests. Special thanks to my sister for accompanying me on the long, otherwise lonely trip to Trieste where I was presenting at a conference.

Thanks to my advisor, Mark Messier, for introducing me to the MIPP experiment and allowing me the freedom to explore my interests. I have learned a great deal from Mark, about physics, the tools used by physicists, working within a group and effectively presenting your work. Without his guidance I would never have made it through what seemed to be an overwhelming task. Many thanks also to my doctoral committee, Brian Serot, Jon Urheim, and Rick Van Kooten for all of their time spent reviewing my dissertation and suggesting revisions.

I am also grateful to all of the MIPP collaborators and the staff at FNAL. Special thanks to Jon Paley for all of his help and constructive criticism, Holger Meyer for coordinating the data taking, and Dan Kaplan and Nick Solomey for introducing this technique to myself and the collaboration as a whole. Without my fellow graduate students, Andrey Lebedev and Sharon Seun, this anaylsis would not have been possible. They each put a great deal of time and effort getting this detector into working order before I even joined 
the experiment, and a great deal more refurbishing it after the fire. Working with so many outstanding physicists has been a wonderful experience that will greatly benefit me moving forward. 
Nicholas J. Graf

MEASUREMENT OF THE CHARGED KAON MASS WITH THE MIPP $\mathrm{RICH}$

The currently accepted value of the charged kaon mass is $493.677 \pm 0.013$ $\mathrm{MeV}(26 \mathrm{ppm})$. It is a weighted average of six measurements, most of which use kaonic atom X-ray energy techniques. The two most recent and precise results dominate the average but differ by 122 ppm. Inconsistency in the data set needs to be resolved, preferably using independent techniques. One possibility uses the Cherenkov effect. A measurement of the charged kaon mass using this technique is presented. The data was taken with the Main Injector Particle Production experiment at Fermi National Accelerator Laboratory using a tagged beam of protons, kaons, and pions ranging in momentum from $37 \mathrm{GeV} / c$ to $63 \mathrm{GeV} / c$. The measured value is $491.3 \pm 1.7$ $\mathrm{MeV}$. This is within $1.4 \sigma$ of the current value. An improvement in precision by a factor of 35 would make this technique competitive for resolving the ambiguity in the X-ray data. 


\section{Contents}

1 Introduction 1

2 Previous $K^{-}$Mass Measurements 5

2.1 Backenstoss . . . . . . . . . . . . . . . . 5

2.2 Cheng ................................ 7

2.3 Barkov ........................ 8

$2.4 \mathrm{Lum} \ldots \ldots \ldots \ldots \ldots$

2.5 Gall ........................ 10

2.6 Denisov . . . . . . . . . . . . . . . . . . . . . . . . . . . 12

3 The MIPP Experiment $\quad 14$

3.1 Experimental Layout . . . . . . . . . . . . . . 15

3.2 Particle Acceptance . . . . . . . . . . . . . . . . . 17

3.3 Particle Identification . . . . . . . . . . . . . . . . 17

3.4 Active Detectors . . . . . . . . . . . . . . . . 18

3.4.1 Beam Cherenkov . . . . . . . . . . . . 18

3.4.2 Drift Chambers . . . . . . . . . . . . 19

3.4.3 Multiwire Proportional Chambers . . . . . . . . 20 
3.4.4 Hadron Calorimeter . . . . . . . . . . . . 20

3.5 Data Acquisition System . . . . . . . . . . . . 21

3.6 Slow Controls . . . . . . . . . . . . . . . . . . . . . . . . 24

3.6.1 Monitoring Systems . . . . . . . . . . . 26

3.6.2 Database Logging . . . . . . . . . . . 27

3.6.3 High Voltage . . . . . . . . . . . 28

4 MIPP Beam Line and Trigger $\quad 29$

4.1 Beam Line . . . . . . . . . . . . . . . . . . . . . . . 29

4.2 Secondary Beam . . . . . . . . . . . . . . . 30

4.2.1 Beam Quality ............... 32

4.3 Trigger ...................... 34

4.3.1 Beam Particle Identification . . . . . . . . . 35

4.3.2 Beam Cherenkov .............. . . 35

4.3.3 End of Spill and Calibration Triggers . . . . . . . 36

4.3.4 Veto Counter .............. 37

$5 \quad$ RICH Detector $\quad 38$

5.1 The Cherenkov Effect . . . . . . . . . . . . . . 38

5.2 Physical Description ................. 41

5.2.1 Entrance and Exit Windows . . . . . . . . . 43

5.2.2 Mirrors .................. . . 43

5.2.3 Phototubes ................... 45

5.2.4 Holder Plate. . . . . . . . . . . . . 47 
5.2.5 High Voltage ............... . . 49

5.2.6 Readout System . . . . . . . . . . . 50

5.2.7 LED Monitoring System . . . . . . . . . . 51

5.3 The RICH in MIPP . . . . . . . . . . . . . . 52

5.3 .1 RICH Fire . . . . . . . . . . . . 53

5.3.2 RICH Slow Controls . . . . . . . . . . 57

6 RICH Mass Measurement Concept $\quad 59$

6.1 Role of RICH Detector . . . . . . . . . . . . . . . . . 59

6.1.1 Basic Mass Measurement Concept . . . . . . . . . 60

6.1.2 Estimated Uncertainty . . . . . . . . . 62

$\begin{array}{lll}7 & \text { Data Reconstruction } & 68\end{array}$

7.1 Tracking ............................ 68

7.1 .1 Straight Line Fits . . . . . . . . . . . . 70

7.2 Wire Chambers . . . . . . . . . . . . . 72

7.2.1 Wire Clusters .............. 72

7.2.2 Wire Crosses ............... . . 73

7.2 .3 Track Segments . . . . . . . . . . . . 74

7.3 Ring Fitting . . . . . . . . . . . . . 76

$\begin{array}{lll}8 & \text { Data } & 78\end{array}$

8.1 Running Conditions . . . . . . . . . . . . 78

8.2 Data Quality ........................ 80 
8.2.1 Magnet Current .............. 80

8.2.2 $\mathrm{CO}_{2}$ Density.................... 81

8.2.3 Finding Bad Channels . . . . . . . . . . . . . 82

8.2.4 PMT Noise Rate . . . . . . . . . . . . . . . . . . 90

8.3 Data Selection . . . . . . . . . . . . . 93

9 Alignment 106

9.1 Chamber Alignment . . . . . . . . . . . . 106

9.2 RICH Mirror Alignment . . . . . . . . . . . . . . 109

$\begin{array}{ll}10 \text { Data Analysis } & 119\end{array}$

10.1 Light Yield in More Detail . . . . . . . . . . . . . . . . 119

10.1.1 Light Smearing . . . . . . . . . . . . 126

10.1.2 Probability of PMT Hit . . . . . . . . . . . 127

10.2 Data Occupancy . . . . . . . . . . . . . . . . 129

10.3 Predicting Occupancy . . . . . . . . . . . . . . . . . 132

10.4 Fitting Procedure . . . . . . . . . . . . . . . 137

10.5 Fit Results . . . . . . . . . . . . . . . . . 140

10.5.1 Systematic Uncertainites . . . . . . . . . . 163

10.6 Final Result and Conclusions . . . . . . . . . . . . . 168

10.6.1 Suggestions for Improvement . . . . . . . . . . . . 171 


\section{List of Figures}

1.1 Current charged kaon mass data set . . . . . . . . . . 3

3.1 Schematic view of the MIPP spectrometer . . . . . . . 16

3.2 Hadron calorimeter schematic . . . . . . . . . . . 22

3.3 Schematic of the data acquisition system . . . . . . . 25

4.1 Fermilab accelerator ............... . . 31

4.2 MIPP beamline .................... 33

4.3 Upstream beam Cherenkov pressure curve summary . . . . 36

4.4 Beam trigger purity measured with the RICH . . . . . . . . 37

5.1 Cherenkov effect . . . . . . . . . . . . . 40

5.2 Schematic of RICH detector . . . . . . . . . . . . 42

5.3 Drawing of RICH mirror layout . . . . . . . . . . . 44

5.4 Drawing of $\mathrm{RICH}$ holder plate . . . . . . . . . . . . 48

5.5 Schematic of RICH electronics . . . . . . . . . . 52

6.1 Optimal fractions for kaon mass measurement . . . . . . . 65

6.2 Expected statistical uncertainty for kaon mass measurement 66 
7.1 Wire plane geometry diagram . . . . . . . . . .

8.1 Stability of momentum selecting magnet current . . . . . 80

8.2 RICH vessel pressure during low momentum running . . . . 82

8.3 RICH vessel temperature during low momentum running . $\quad 83$

8.4 RICH $\mathrm{CO}_{2}$ density during low momentum running . . . . . 84

8.5 RICH vessel pressure during high momentum running . . . 85

8.6 RICH vessel temperature during high momentum running . 86

8.7 RICH $\mathrm{CO}_{2}$ density during high momentum running $\ldots . \quad 87$

8.8 In-spill PMT occupation . . . . . . . . . . . . . . 88

8.9 PMT occupation for calibration events . . . . . . . 88

8.10 Number of RICH bad channels . . . . . . . . . . . . . 89

8.11 Number of bad channels vs. run during low momentum kaon mass data taking . . . . . . . . . . . . . . . . . 89

8.12 Number of bad channels vs. run during high momentum kaon mass data taking $\ldots \ldots \ldots \ldots$

8.13 RICH event display. . . . . . . . . . . . . . . . . . . 91

8.14 Number of RICH hot columns . . . . . . . . . . . . . 92

8.15 Hot column fraction . . . . . . . . . . . . . . . . . 92

8.16 FEU occupation for calibration events . . . . . . . . 93

8.17 Hamamatsu occupation for calibration events . . . . . . . 94

8.18 Hcal ADC sum for $40 \mathrm{GeV}$ protons . . . . . . . . . . 95

8.19 Hcal ADC sum for $40 \mathrm{GeV}$ kaons . . . . . . . . . . . 96 
8.20 Hcal ADC sum for $40 \mathrm{GeV}$ pions . . . . . . . . . . . . 96

8.21 Hcal ADC sum for $60 \mathrm{GeV}$ protons . . . . . . . . . . . 97

8.22 Hcal ADC sum for $60 \mathrm{GeV}$ kaons . . . . . . . . . . . . . . . 97

8.23 Hcal ADC sum for $60 \mathrm{GeV}$ pions . . . . . . . . . . . . . . . 98

8.24 Track segment $\chi^{2}$ for the $40 \mathrm{GeV}$ data set . . . . . . . . . 100

8.25 Track segment slope for the $40 \mathrm{GeV}$ data set . . . . . . . . 101

$8.26 \chi^{2}$ comparison for $40 \mathrm{GeV}$ tracks . . . . . . . . . . 102

8.27 Track slopes for $40 \mathrm{GeV}$ tracks . . . . . . . . . . . 103

9.1 Chamber residuals . . . . . . . . . . . . . . 108

$9.2 \quad$ DC123 z-alignment . . . . . . . . . . . . . 110

9.3 DC123 z-alignment without DC3 rotation . . . . . . . . 111

9.4 Number of PMT hits in ring fits . . . . . . . . . . . 113

$9.5 \quad$ Fitted ring radius . . . . . . . . . . . . . . . . . 114

$9.6 \quad \chi^{2}$ per degree of freedom for ring fits . . . . . . . . . . . 114

9.7 Mirror 8 horizontal alignment . . . . . . . . . . . 115

9.8 Mirror 8 vertical alignment . . . . . . . . . . . . . 115

9.9 Mirror 9 horizontal alignment . . . . . . . . . . . . 116

9.10 Mirror 9 vertical alignment . . . . . . . . . . . . . 116

9.11 Projected ring center for $40 \mathrm{GeV}$ tracks . . . . . . . . . . 117

9.12 Mirror 8 vs. Mirror 9 ring center for $40 \mathrm{GeV}$ tracks . . . . . 118

10.1 Refractive index of carbon dioxide as a function of wavelength at $\mathrm{STP} \ldots \ldots \ldots \ldots \ldots \ldots$ 
10.2 Absorption coefficient of carbon dioxide . . . . . . . . . . 121

10.3 Reflectance of RICH mirrors . . . . . . . . . . . . . 121

10.4 RICH Hamamatsu photomultiplier tube efficiency . . . . . 122

10.5 RICH quartz window transmission . . . . . . . . . . 122

10.6 Reflectance of RICH PMT cones . . . . . . . . . . . . . 123

10.7 Phototube geometric acceptance. . . . . . . . . . . 124

10.8 Npe vs. wavelength for $60 \mathrm{GeV}$ pion. . . . . . . . . 125

10.9 Npe vs. angle for $60 \mathrm{GeV}$ pion. . . . . . . . . . . 128

10.10 Hit map for $40 \mathrm{GeV}$ protons . . . . . . . . . . . . . . 131

10.11 Proton momentum spectrum from FLUKA simulation of $\mathrm{Cu}$

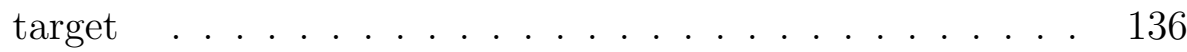

10.12 Kaon momentum spectrum from FLUKA simulation of $\mathrm{Cu}$

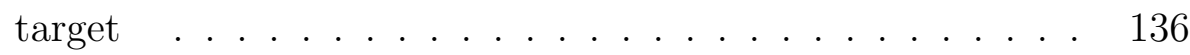

10.13 Pion momentum spectrum from FLUKA simulation of $\mathrm{Cu}$

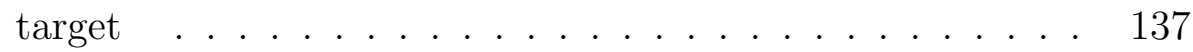

10.14 Relative momentum deviation from FLUKA . . . . . . . 138

$10.15 \chi^{2}$ contour for $\sigma_{0} \ldots \ldots \ldots \ldots$

$10.16 \chi^{2}$ contour for $\sigma_{N} \ldots \ldots \ldots \ldots$. . . . . . . . . . . 149

$10.17 \chi^{2}$ contour for density scaling factor . . . . . . . . . . . 149

$10.18 \chi^{2}$ contour for pion momentum . . . . . . . . . . 150

$10.19 \chi^{2}$ contour for kaon mass . . . . . . . . . . . . . 150

10.20 Occupancy fits for $37 \mathrm{GeV}$ data set, mirror 9 only . . . . . 151

10.21 Occupancy fits for $37 \mathrm{GeV}$ data set, mirror 8 only . . . . . 152 
10.22 Occupancy fits for $40 \mathrm{GeV}$ data set, mirror 9 only . . . . 153

10.23 Occupancy fits for $40 \mathrm{GeV}$ data set, mirror 8 only . . . . . 154

10.24 Occupancy fits for $42 \mathrm{GeV}$ data set, mirror 9 only . . . . . 155

10.25 Occupancy fits for $42 \mathrm{GeV}$ data set, mirror 8 only . . . . 156

10.26 Occupancy fits for $56 \mathrm{GeV}$ data set, mirror 9 only . . . . . 157

10.27 Occupancy fits for $56 \mathrm{GeV}$ data set, mirror 8 only . . . . 158

10.28 Occupancy fits for $60 \mathrm{GeV}$ field off data set, mirror 9 only 159

10.29 Occupancy fits for $60 \mathrm{GeV}$ field off data set, mirror 8 only 160

10.30 Occupancy fits for $60 \mathrm{GeV}$ field on data set, mirror 9 only 161

10.31 Occupancy fits for $60 \mathrm{GeV}$ field on data set, mirror 8 only 162

10.32 Occupancy fits for $63 \mathrm{GeV}$ data set, mirror 9 only . . . . . 164

10.33 Ooccupancy fits for $63 \mathrm{GeV}$ data set, mirror 8 only . . . . 165

10.34 Fit of mass results for low momentum sets . . . . . . . . 169

10.35 Fit of mass results for high momentum data sets . . . . . . 170

10.36 Final mass fit . . . . . . . . . . . . . . 171 


\section{List of Tables}

8.1 Data totals before cuts. . . . . . . . . . . 79

8.2 Summary of cuts for the $37 \mathrm{GeV}$ data set. . . . . . . . . 99

8.3 Summary of cuts for the $40 \mathrm{GeV}$ data set . . . . . . . . . 100

8.4 Summary of cuts for the $42 \mathrm{GeV}$ data set . . . . . . . . . . . 101

8.5 Summary of cuts for the $56 \mathrm{GeV}$ data set . . . . . . . . . 102

8.6 Summary of cuts for the $60 \mathrm{GeV}$ field off data set . . . . . . 103

8.7 Summary of cuts for the $60 \mathrm{GeV}$ field on data set . . . . . . 104

8.8 Summary of cuts for the $63 \mathrm{GeV}$ data set . . . . . . . . . . 104

8.9 Data totals after cuts. . . . . . . . . . . 105

10.1 List of fit parameters _. . . . . . . . . . . . 139

$10.2 \chi^{2}$ and number of degrees of freedom . . . . . . . . . 140

10.3 Average and RMS angles for pion occupation distributions 141

10.4 Average and RMS angles for proton occupation distributions 143

10.5 Average and RMS angles for kaon occupation distributions 144

10.6 Summary of fit results for all parameters . . . . . . . . . 146

10.7 Parameter correlations for $40 \mathrm{GeV}$ mirror 9 fit . . . . . . 147

10.8 Summary of kaon mass fit results . . . . . . . . . . . . . 166 
10.9 Parameter uncertainties and first derivatives . . . . . . . . 167

10.10Breakdown of systematic error estimates . . . . . . . . 167 


\section{Chapter 1}

\section{Introduction}

An improved charged kaon mass value would have implications for the value of the CKM matrix element $\mathrm{V}_{\text {us }}$ as determined from measurements of the branching ratio of

$$
K^{+} \rightarrow \pi^{0} e^{+} \nu
$$

referred to as $K_{e 3}^{+}$. This method has a smaller theoretical uncertainty than determination from hyperon decays due to less need for hadronic physics [37]. The branching ratio is proportional to the mass to the fifth power, making it sensitive to uncertainty in the mass.

The currently accepted value of the charged kaon mass as reported by the PDG is $493.677 \pm 0.013 \mathrm{MeV}, \chi^{2}=22.9$ for 5 degrees of freedom, with a probability of $0.04 \%$ [13]. This value is a weighted average of six different measurements. The uncertainty of 26 ppm has been scaled by a factor of 2.4 due to a large discrepancy between input values, as evidenced by the high 
$\chi^{2}$ and low probability.

The source of this discrepancy is mainly due to the measurements of kaonic atom X-ray energies by Denisov [11] and Gall [20], which differ by $122 \mathrm{ppm}$, or $4.6 \sigma$. These two are the most recent as well as the most precise results available. As such, they dominate the overall average, resulting in a binodal probability distribution function as shown in Figure 1.1.

The Gall measurement itself is a weighted average of four kaonic atom transitions: $K^{-} \mathrm{Pb}(9 \rightarrow 8), K^{-} \mathrm{Pb}(11 \rightarrow 10), K^{-} \mathrm{W}(9 \rightarrow 8), K^{-} \mathrm{W}(11 \rightarrow$ $10)$. The $K^{-} \mathrm{Pb}(9 \rightarrow 8)$ value is lower than the others and is responsible for some internal inconsistency in the Gall data set as well as the inconsistency in the overall average. A potential cause could be contaminant $\gamma$ rays, to which the Denisov measurement is less sensitive. According to [13], removing the Gall $K^{-} \mathrm{Pb}(9 \rightarrow 8)$ data point produces a more consistent data set with a significantly higher probability. However, removing other points such as Denisov result in acceptable probabilities. The conclusion, then, is that there is no clear reason to reject this data.

The internal inconsistency in the data set needs to be resolved with further measurements, ideally using independent techniques. This thesis explores one possibility: a precision measurement of the charged kaon mass using a Ring Imaging Cherenkov (RICH) detector. Use of a RICH detector is novel in precision mass measurements.

The opportunity to explore this technique presented itself at the end of run of the Main Injector Particle Production (MIPP) experiment. MIPP 


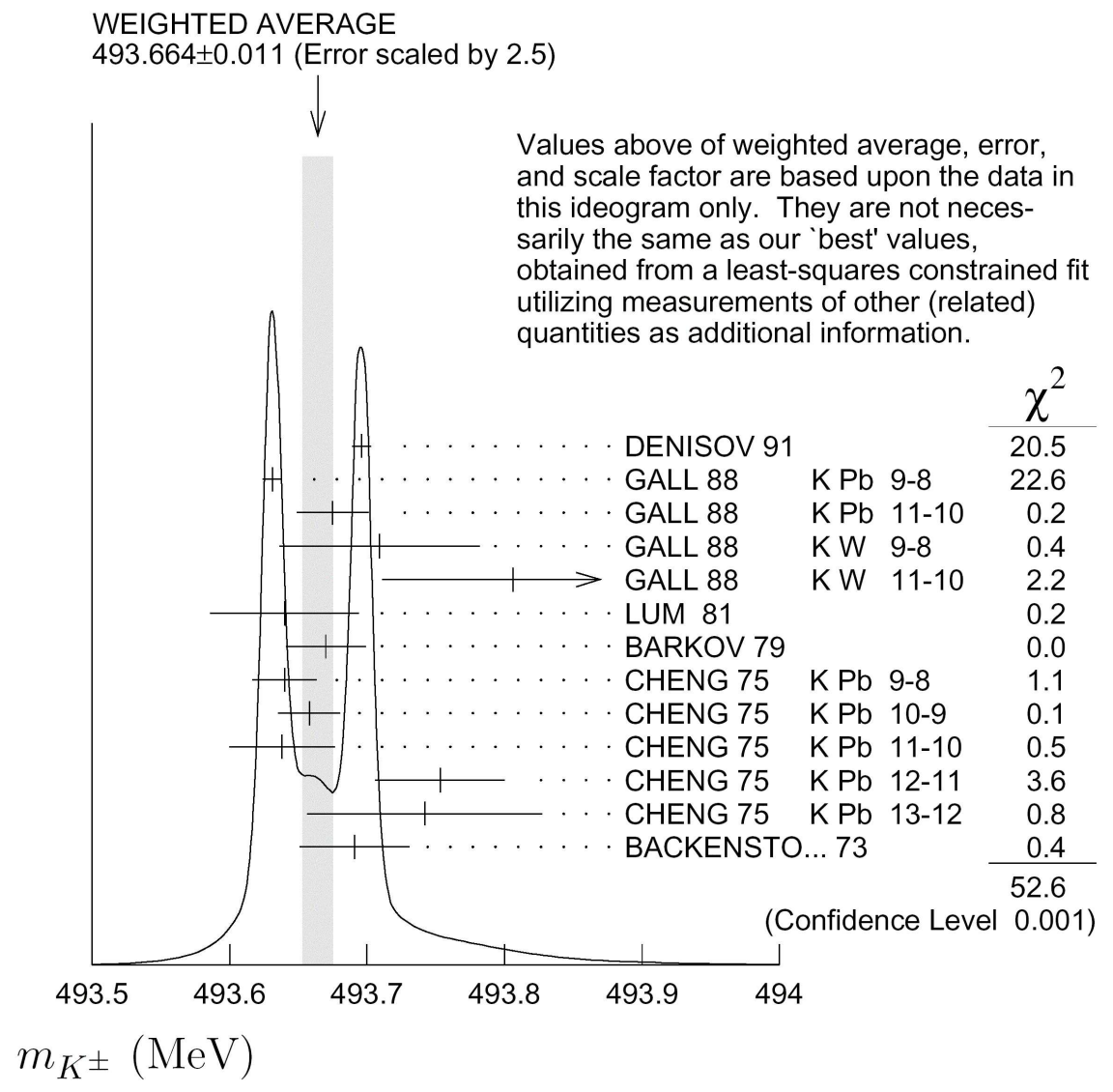

Figure 1.1: Current charged kaon mass data set from [13]. 
used a beam of pions, kaons, and protons with a momentum bite on the order of $5 \%$. This made it uniquely suited to explore the possibility of kaon mass measurements using a RICH detector. 


\section{Chapter 2}

\section{Previous $K^{-}$Mass}

\section{Measurements}

This chapter summarizes the methods and results of previous measurements of the charged kaon mass. Most measure the energy of X-rays given off by transitions in kaonic atoms. Kaonic atoms are created by stopping a beam of negatively charged kaons in a target, creating a hydrogen-like atom with a kaon instead of an electron.

\section{$2.1 \quad$ Backenstoss}

The Backenstoss measurement, described in [3], was made using a $K^{-}$beam at the CERN proton synchrotron. The negatively charged kaons were stopped in $\mathrm{Au}$ and $\mathrm{Ba}$ targets. The energy of X-rays from kaonic atom circular 
$(l=n-1)$ transitions was measured using each of two Ge-Li semiconductor detectors. The X-ray energies can then be related to the charged kaon mass. The transitions used were: $K^{-} \mathrm{Au}(12 \rightarrow 11), K^{-} \mathrm{Au}(11 \rightarrow 10), K^{-} \mathrm{Au}(10 \rightarrow$ 9), $K^{-} \mathrm{Au}(9 \rightarrow 8), K^{-} \mathrm{Ba}(11 \rightarrow 10), K^{-} \mathrm{Ba}(10 \rightarrow 9), K^{-} \mathrm{Ba}(9 \rightarrow 8)$, and $K^{-} \mathrm{Ba}(8 \rightarrow 7)$. They were chosen because the effects of strong interactions was small.

Calibration was performed for each target run. For the Ba target run, $\gamma$ rays from ${ }^{192} \mathrm{Ir}$ and $K_{\alpha 1}$ and $K_{\alpha 2} \mathrm{X}$-rays from Os and Pt were used. Calibration for the Au target running used X-rays from ${ }^{75} \mathrm{Se}$ and ${ }^{198} \mathrm{Au}$.

Contaminant X-rays from non-circular transitions needed to be removed from the energy spectrum. To do this, calculations of the atomic cascade were made to determine the relative intensities of these transitions. Final Xray energies were determined from fits to the spectrum and averaged for both detectors, with uncertainties for statistics, fitting procedure, background subtraction, and calibration.

The kaon mass was then determined by comparing measured energies to theoretical values assuming a mass value. In this case, $493.75 \mathrm{MeV}$ was assumed. This calculation was done by numerically integrating the Klein-Gordon equation while taking into account nuclear charge distribution, strong interaction effects, vacuum polarization to seventh order, polarization of the nucleus, electron screening and dynamic polarization of the electron cloud. The dominant error in this calculation was due to electron screening. To first order, transition energies are proportional to mass. A linear rela- 
tionship is assumed between the calculated and measured energy difference and the calculated energy. A least squares fit was done to determine the slope, which then sets the mass scale. The final value obtained is $493.691 \pm 0.040$ $\mathrm{MeV}$.

\subsection{Cheng}

The Cheng measurement [8] also used energy measurements of X-rays from circular transitions in kaonic atoms. The method is very similar to that of Backenstoss. In this case, the transitions measured were: $K^{-} \mathrm{Pb}(13 \rightarrow 12)$, $K^{-} \mathrm{Pb}(12 \rightarrow 11), K^{-} \mathrm{Pb}(11 \rightarrow 10), K^{-} \mathrm{Pb}(10 \rightarrow 9)$, and $K^{-} \mathrm{Pb}(9 \rightarrow 8)$. The measurement was performed at the Brookhaven AGS using a proton beam with tungsten and iridium targets to produce a $750 \mathrm{MeV} / c K^{-}$beam. A $17.5 \mathrm{~cm}$ copper degrader was used to bring kaons to rest in a lead target.

X-rays energies were measured by means of a $\mathrm{Ge}(\mathrm{Li})$ detector. Calibration used X-rays from ${ }^{75} \mathrm{Se}$ and ${ }^{198} \mathrm{Au} . K_{\alpha 1}$ and $K_{\alpha 2}$ X-rays from lead as well as $e^{+}$annihilation lines were used as a cross check on the calibration.

As in other kaonic X-ray measurements, contaminants from non-circular transitions must be accounted for. Relative intensities of these peaks were calculated from atomic cascade simulations which included effects of strong interactions. Measured higher-order transitions were used to check calculations, which were estimated to within $20 \%$. When fitting the energy spectrum, these peaks were modeled as Gaussian with positions and relative 
intensities fixed.

The kaon mass was determined as in Backenstoss [3] by assuming a linear relationship between the calculated and measured energy difference and the calculated energy, then performing a least squares fit. Energies were calculated from the Klein Gordon equation for an assumed $K^{-}$mass value, a point nucleus, and accounting for vacuum polarization to seventh order, relativistic recoil, screening for two 1S electrons, and nuclear polarization. The final value obtained is $493.657 \pm 0.020 \mathrm{MeV}$.

\subsection{Barkov}

The method employed by Barkov [5] is different from methods previously described. Carried out at the VEPP 2M storage ring, the charged kaon mass was measured from the reaction $e^{+} e^{-} \rightarrow \phi \rightarrow \mathrm{K}^{+} \mathrm{K}^{-}$. Beam energy was determined from measurement of the spin precession frequency by resonance beam depolarization with a high frequency longitudinal magnetic field. Kaon kinetic energy was determined using nuclear emulsion range measurements.

A stainless steel vacuum tube $36 \mathrm{~mm}$ in diameter defined the interaction region. Kaons emerge primarily perpendicular to the beam axis and pass into two symmetrically placed stacks of nuclear emulsion BR-2. Uncertainty on the range measurement was mainly due to the precision of thickness measurements. The range-energy relation was calibrated using $40 \mathrm{MeV}$ protons. Also contributing to uncertainty of the kaon kinetic energy were energy spread of 
particles in the storage ring, fluctuations of kaon energy loss, dispersion in the emulsion chamber, and asymmetry in the kaon energy distribution which was calculated from radiative effects.

The final value of the charged kaon mass, averaged over two runs, was $493.670 \pm 0.029 \mathrm{MeV}$.

\subsection{Lum}

The Lum [32] measurement used the $6 h \rightarrow 5 g$ transition in potassium. Determining the X-ray energy, however, relied on a different technique than the previously described measurements. The energy of this transition lies along the $\mathrm{K}$ absorption edge of erbium. Measuring the X-ray attenuation in erbium was used to find the energy.

The experiment ran at Lawrence Berkeley Laboratory using $5.3 \mathrm{GeV}$ protons and a tungsten target to produce a $450 \mathrm{MeV} K^{-}$beam. X-rays were detected using each of four Ge semiconductor detectors. Three erbium absorbers of varying thickness were mounted on a rotatable wheel between the detectors and the target, so that each could be covered in turn by any one of the three absorbers or left uncovered.

Background X-ray levels were determined using ${ }^{341} \mathrm{Am} \gamma$ rays to generate erbium fluorescent peaks. Relative intensities of non-circular transitions were calculated using simulations of the atomic cascade. A precise determination of the $\mathrm{K}$ absorption edge for all three foils was made at NBS using two flat 
silicon crystals, a two axis spectrometer and a tungsten X-ray tube for the radiation source. Absorption profiles are then determined from measurements of three configurations: the two crystals parallel and anti-parallel with and without the erbium absorber.

The actual potassium $6 h \rightarrow 5 g$ transition energy measurement was made in two runs. The dominant errors were from X-ray counting statistics and the contribution of non-circular transitions. As in the other measurements that used X-rays, a linear fit between calculated energy and and the difference from the measured energy using the method of least squares. Energies were calculated from the Klein Gordon equation assuming the then current PDG value of $K^{-}$mass, $493.669 \pm 0.018 \mathrm{MeV}$, and accounting for the coulomb potential, finite nuclear charge distribution, strong interaction potential, vacuum polarization, electron screening, nuclear polarization, and nuclear center of mass motion effects. The final value obtained was $493.640 \pm 0.054 \mathrm{MeV}$.

\subsection{Gall}

The Gall measurement [20] also used energy measurements of X-rays from circular transitions in kaonic atoms. As a result, the method is very similar to the other kaonic X-ray measurements already described. In this case, the transitions measured were: $K^{-} \mathrm{Pb}(11 \rightarrow 10), K^{-} \mathrm{Pb}(9 \rightarrow 8), K^{-} \mathrm{W}(11 \rightarrow$ 10), and $K^{-} \mathrm{W}(9 \rightarrow 8)$. These transitions were chosen because of negligible strong interaction effects. 
The measurement was performed at the Brookhaven AGS using a 680 $\mathrm{MeV} / c K^{-}$beam slowed by a copper degrader. X-ray energies were measured using three Ge detectors which were continuously calibrated using ${ }^{57} \mathrm{Co}$, ${ }^{133} \mathrm{Ba},{ }^{192} \mathrm{Ir}$, and ${ }^{137} \mathrm{Cs}$ sources. As in other kaonic X-ray measurements, contaminants from non-circular transitions were accounted for by calculating relative intensities of these peaks from atomic cascade simulations.

For each target, one spectrum for each of the three detectors was taken. When fitting the energy spectrum, the position and relative intensities of the non-circular transitions were held fixed at the values obtained from the cascade simulation. Additional $\gamma$ and X-ray lines were represented as Gaussian.

A linear fit was done between calculated energy and the difference of calculated and measured energies. Energy was calculated from the Klein Gordon equation for an assumed $K^{-}$mass value, accounting for vacuum polarization, relativistic recoil, electron screening, nuclear polarization, and finite nuclear charge distribution. The $K^{-}$mass was determined iteratively by starting with an assumed value and scaling by the ratio of measured to calculated energies. Studies of systematic errors were done for detector resolution, calculated energy levels, non-circular transitions, and contaminant nuclear $\gamma$ rays.

The mass values obtained for each transition were: $493.675 \pm 0.026 \mathrm{MeV}$ for $K^{-} \mathrm{Pb}(11 \rightarrow 10), 493.631 \pm 0.007 \mathrm{MeV}$ for $K^{-} \mathrm{Pb}(9 \rightarrow 8), 493.806 \pm 0.095$ $\mathrm{MeV}$ for $K^{-} \mathrm{W}(11 \rightarrow 10)$, and $493.709 \pm 0.073 \mathrm{MeV}$ for $K^{-} \mathrm{W}(9 \rightarrow 8)$. The average value was $493.636 \pm 0.011 \mathrm{MeV}$ with a scale factor of 1.5 on the final 
uncertainty, to account for the inconsistency of the $K^{-} \mathrm{Pb}(9 \rightarrow 8)$ transition which, as previously discussed, is the primary source of disagreement between the input results for the PDG value, along with the Denisov measurement.

\subsection{Denisov}

The Denisov measurement [11] makes use of a single kaonic X-ray transition, namely the $4 f \rightarrow 3 d$ transition in ${ }^{12} \mathrm{C}$. Using a high resolution Cauchois crystal diffraction spectrometer and the choice of a light nucleus resulted in this measurement being less susceptible to contaminant $\gamma$ rays. Other reasons why this transition was chosen were negligible electron screening, there was only a slight perturbation from strong interaction effects, and the transition energy lies in a region of optimal signal to noise ratio for the detector used.

The target used to capture slow $K^{-}$was graphite with layers of copper and molybdenum. The purpose of the copper layer was to intensify the Xrays, while molybdenum weakened bremsstrahlung. X-rays were detected using a $\mathrm{Ge}(\mathrm{Li})$ detector. Calibration was done with a ${ }^{182} \mathrm{Ta} \gamma$ source and silver foil, making use of the $K_{\alpha 1}$ and $K_{\alpha 2}$ lines of silver.

Kaon mass was determined by assuming a linear relationship between the calculated and measured energy difference and the calculated energy. Energies were calculated from the Klein Gordon equation for an assumed $K^{-}$mass value, accounting for finite nuclear charge distribution, coulomb interaction potential, vacuum polarization to third order, strong interactions, 
a reduced mass correction, nuclear polarization, electron screening, nuclear recoil, and Lamb shift. The dominant errors given for this calculation were due to electron screening, nuclear charge distribution, and strong interaction potential. The final mass value was $493.6960 \pm 0.0059 \mathrm{MeV}$. Along with the Gall measurement, the Denisov result is the primary source of disagreement between the input results for the PDG value. 


\section{Chapter 3}

\section{The MIPP Experiment}

The data presented in this thesis was collected at Fermi National Accelerator Laboratory (FNAL) during January and February 2006 using the Main Injector Particle Production (MIPP) spectrometer. This chapter discusses the beamline, detector hardware, operation, and physics goals of the experiment.

MIPP used protons from the Main Injector to produce a secondary hadron beam. High statistics measurements were made of the final state of interac-

tions of $\pi^{ \pm}, K^{ \pm}, p$ and $\bar{p}$ ranging in momentum from $5-85 \mathrm{GeV} / c$ with a variety of nuclear targets, such as $\mathrm{C}, \mathrm{Bi}, \mathrm{Be}, \mathrm{Al}$, and liquid hydrogen. MIPP also performed a service measurement of production off the NuMI target using $120 \mathrm{GeV} / c$ Main Injector protons.

The MIPP data is applicable to many different areas of physics. Hadron nucleus interaction data used as inputs for Monte Carlo generators such as FLUKA, MARS, and GEANT is quite sparse. The broad survey of nuclear 
targets and momenta in MIPP will greatly improve this input data set. The MIPP data is also useful for designing neutrino beams, atmospheric neutrino flux calculations, relativistic heavy ion physics, and nuclear physics. The analysis of the NuMI target data will greatly reduce systematic errors in neutrino flux calculations for MINOS.

\subsection{Experimental Layout}

In order to achieve the goals of the experiment, high precision particle identification of produced species over the entire kinematic range provided by the Main Injector was needed. MIPP was able to achieve particle identification for $\pi, K$, and $p$ over the range $0.1-80 \mathrm{GeV} / c$ using mostly existing components from previous experiments.

The layout of the experiment is shown in Figure 3.1. It used two magnetic spectrometers arranged in series: the Jolly Green Giant and the Rosie magnet. They covered the complete range $P, P_{\mathrm{T}}$ of particle production providing unambiguous particle identification and 5\% momentum resolution. The secondary target is just upstream of the Time Projection Chamber (TPC), which sat inside the magnetic field produced by the Jolly Green Giant. Immediately upstream was a series of 4 drift chambers and a Time of Flight wall. The second spectrometer, which had a stronger dipole magnet, sat upstream and provided particle identification up to beam energies and tracking using two multi-wire proportional chambers and a ring imaging Cherenkov 


\section{MIPP \\ Main Injector Particle Production Experiment (FNAL-E907)}

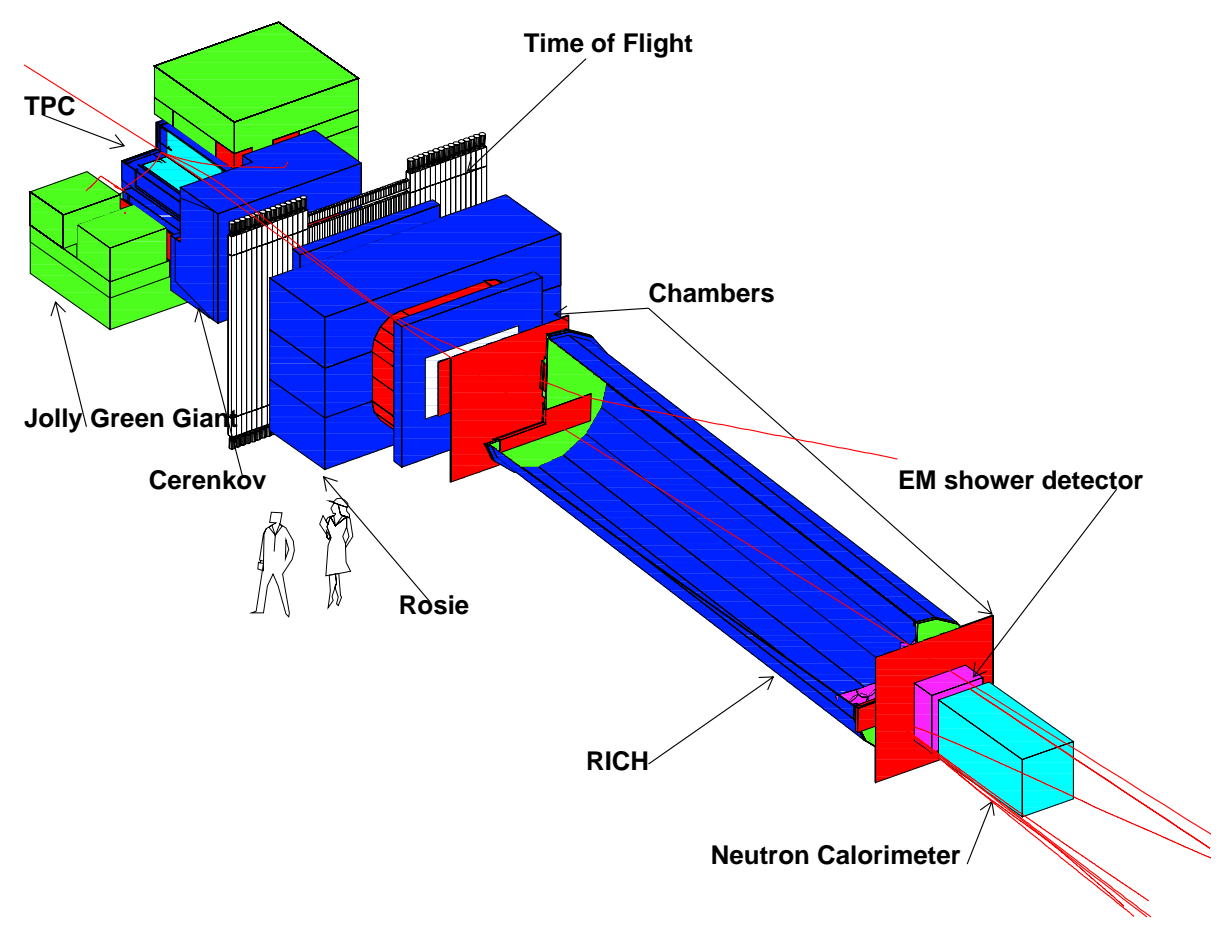

Figure 3.1: Schematic view of the MIPP spectrometer.

detector. Last in line were electromagnetic and hadronic calorimeters.

A wheel apparatus which could be rotated remotely to one of several positions was used to easily select between different targets. One position was always left with no target installed to easily switch to target-out data taking. Since the charged kaon mass measurement requires only uninteracted beam particles, the target wheel was left in the empty position during data taking for this measurement. 


\subsection{Particle Acceptance}

The TPC provides almost complete acceptance for tracks above $p=0.1$ $\mathrm{GeV} / c$ and angles up to 80 degrees, while the acceptance of the threshold Cerenkov starts at $p=0.5 \mathrm{GeV} / c$ up to angles of 15 degrees. The second spectrometer acceptance is restricted to angles less than 5 degrees, with particles above $p=5 \mathrm{GeV} / c$ hitting the wire chamber before the RICH, and the RICH itself seeing particles above $p=10 \mathrm{GeV} / c$. The Time-Of-Flight (TOF) acceptance begins at $1 \mathrm{GeV} / c$ up to angles of 6 degrees.

\subsection{Particle Identification}

Particle identification for the MIPP detector is nearly continuous. Measurements of $\mathrm{d} E / \mathrm{d} x$ in the TPC allow for $\pi / K$ separation below $0.8 \mathrm{GeV} / c$ and $K / p$ separation up to $1.1 \mathrm{GeV} / c$. The threshold Cherenkov counter provides particle identification between $2.7-17 \mathrm{GeV} / c$. The TOF wall fills the range in between, $0.7-2.7 \mathrm{GeV} / c$. Finally, high momentum particle ID is handled by a RICH, which provides $3 \sigma \pi / K$ separation up to $80 \mathrm{GeV} / c$, and $K / p$ separation up to momenta exceeding $120 \mathrm{GeV} / c$. With the current setup, pions and kaons are not identified above $80 \mathrm{GeV} / c$. There is some proton/kaon ambiguity between 4.6 and $7.5 \mathrm{GeV} / c$, and the region around $1 \mathrm{GeV} / c$ has poor coverage. 


\subsection{Active Detectors}

This section focuses on describing in more detail those systems other than the RICH that were active during data taking for the charged kaon mass measurement. The RICH is described in greater detail in Chapter 5 .

\subsubsection{Beam Cherenkov}

The two beam Cherenkov counters are used to tag the PID of incoming beam particles. Each is a cylindrical radiator volume about 18 inches in diameter with a head on the downstream end. Each head contains a mirror which reflects light onto a second mirror. This mirror contains a hole which allows light below a cutoff angle to reach a photomultiplier tube (PMT) situated behind the second mirror, referred to as the inner PMT. Light above the cutoff angle is reflected to the outer PMT. The cutoff angles for the upstream and downstream counters are 5 and 7 mrad respectively. The radiator lengths are 22.9 and $12.2 \mathrm{~m}$. This allows for both inner PMTs to see the same number of photoelectrons statistically.

Gas densities in each counter are chosen so that light from each particle type is seen in a different PMT. Nitrogen is used in both counters for momenta above $30 \mathrm{GeV} / \mathrm{c}$. The upstream counter is used for $\pi / K$ separation by setting the kaon Cherenkov angle to 5 mrad so that it hits the inner PMT. Pions at the same momentum produce light at larger angles which is reflected into the outer PMT. Protons do not radiate under these conditions. 
The downstream counter is used to identify protons. Its density is set so that proton Cherenkov light hits the inner PMT but not the outer.

\subsubsection{Drift Chambers}

A series of three small drift chambers positioned between the downstream beam Cherenkov counter and the target are used to track incoming beam particles. They were used previously by FNAL E690 [9]. They cover a longitudinal distance of $37 \mathrm{~m}$, providing $150 \mu \mathrm{m}$ position resolution and 5 $\mu \mathrm{rad}$ angular resolution. The active area is $15.24 \mathrm{~cm} \times 10.16 \mathrm{~cm}$ with a wire spacing of $1.016 \mathrm{~mm}$ which allows multiple tracks to be resolved. Each

chamber has a total of 160 wires. TDC information provides a measure of arrival time of the track relative to the trigger signal. The gas used is a mixture of $\mathrm{Ar} / \mathrm{C}_{4} \mathrm{H}_{10} /$ methylal in proportions of 82:15:3.

Tracking beyond the TPC through the ROSIE magnet is provided by four larger drift chambers with cover the magnet aperture. These chambers were also used by FNAL E690. The most upstream chamber, DC1, is larger than the other three with an active area of $182.88 \mathrm{~cm} \times 121.92 \mathrm{~cm}$, wire spacing of $3.4925 \mathrm{~mm}$, and 512 wires. The other three chambers have an active area of $152.40 \mathrm{~cm} \times 101.60 \mathrm{~cm}$, wire spacing of $3.175 \mathrm{~mm}$, and 448 wires. Each chamber has four planes of wires. The same gas mixture is used as for the beam chambers, only in proportions of 69:28:3.

Drift time is measured by 32 channel LeCroy 4290 TDC modules organized in 15 CAMAC crates of 22-25 modules, each crate read out by a 
dedicated crate controller. The resolution on the drift time measurement with this system is $1 \mathrm{~ns}$. Other electronic modules used for chamber readout are preamplifiers and Nevis discriminators.

\subsubsection{Multiwire Proportional Chambers}

Two multiwire proportional chambers (PWC) are situated at the upstream and downstream ends of the RICH, extending tracking through the rest of the spectrometer. They were used previously in NA24 at CERN and SELEX at FNAL. Construction is documented in [12]. Each chamber has an active area of $200 \mathrm{~cm} \times 200 \mathrm{~cm}$, wire spacing of $3 \mathrm{~mm}$, and a total of 640 wires in four planes. Two of the planes in PWC5, however, could not hold high voltage and were inactive during data taking. The gas used is $\mathrm{Ar} / \mathrm{CH}_{4} / \mathrm{CF}_{4}$ in ratios of 76.5:8.5:15.

Chamber readout is handled by RMH electronics [30] designed at CERN. The electronics are housed in 8 crates with 20 32-channel cards each.

\subsubsection{Hadron Calorimeter}

Forward-going neutron production is measured by a hadron calorimeter. It is the final detector in the spectrometer and also functions as a beam dump. It was previously used in HyperCP [7]. Most of the active region is composed of lead sheets. The energy deposited by a hadronic shower initiated by a track reaching the calorimeter is summed. Sandwiched in between the lead 
sheets are four scintillator plates which are segmented into two readout cells each symmetric about the beam axis. The scintillator sheets are readout by PMTs, one for each cell A schematic of a cell is shown in Figure 3.2.

The electronics used to read out the calorimeter were designed at the University of Virginia. They consist of an ADC driver to amplify the PMT signals which are then digitized by a 16-bit CAMAC ADC.

\subsection{Data Acquisition System}

This section provides an overview of the MIPP data acquisition system (DAQ). A more detailed discussion can be found in [27].

The MIPP DAQ was designed to read out all detector systems at a minimum rate of $60 \mathrm{~Hz}$ with an average event size of $100 \mathrm{kB}$ for beam delivered in 1 second spills at 20 spills per minute. The rate and event size were set by the TPC which takes longer to read out than all other detectors in the experiment. The actual data rate did not exceed fifteen $600 \mathrm{~ms}$ spills per minute, which the DAQ had no trouble handling.

Figure 3.3 shows a schematic of DAQ, which consisted of:

- A DAQ server connected to public and private networks

- Six VME PowerPCs (PPC) connected to the DAQ server through 100 Mbit network with access to VME and CAMAC read out electronics

- Six VirGen cards built to generate VME interrupts 


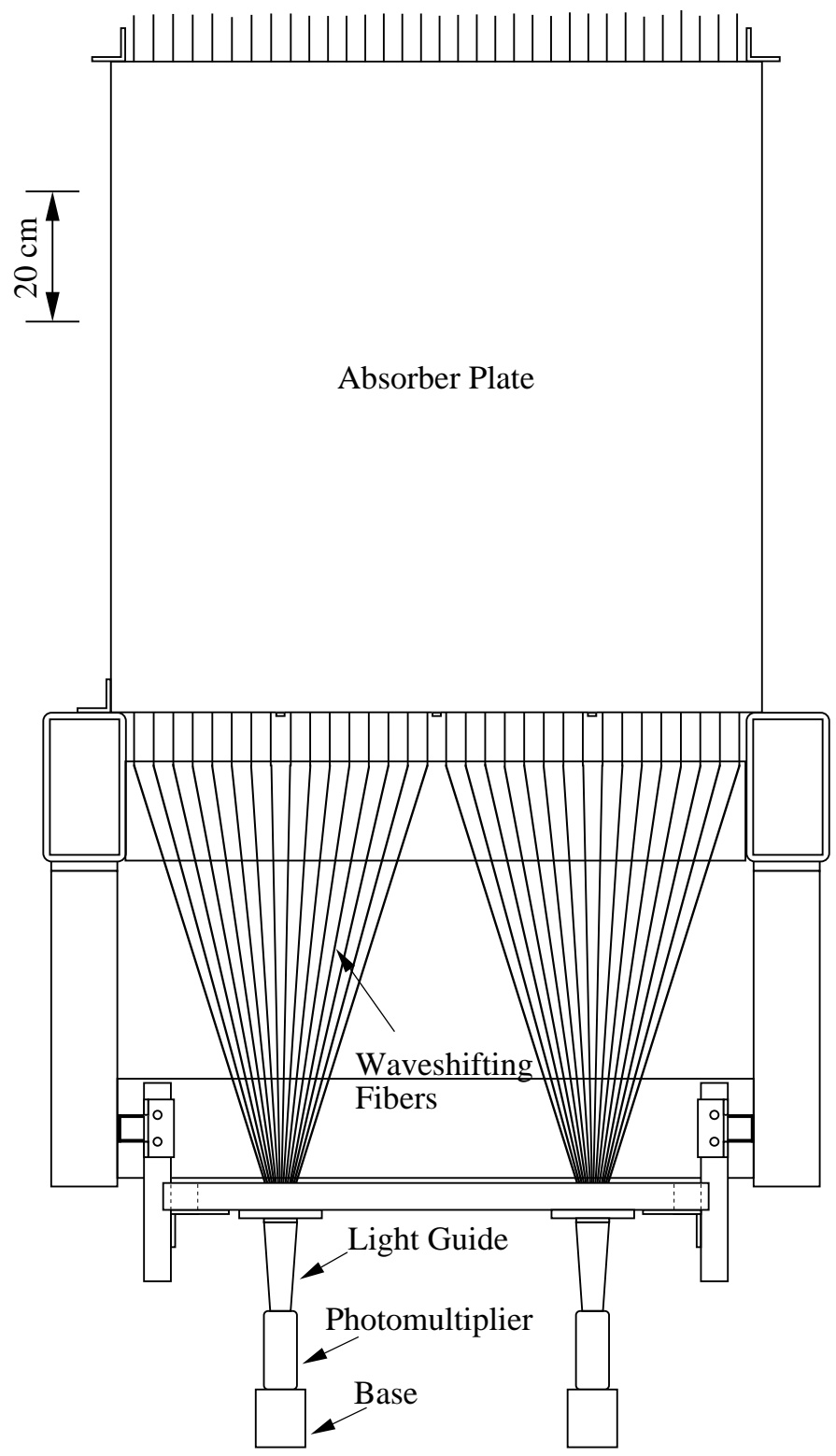

Figure 3.2: Hadron calorimeter schematic. 
- Two CBD 8210 VME CAMAC read out cards

- A computer running online monitoring software

- A dedicated machine for controlling and monitoring all TPC and PMT high voltages (excluding the RICH).

- A database server running PostgreSQL for storage of all online information

Data read out was handled by PPCs, each with $350 \mathrm{MHz}$ processors, $256 \mathrm{MB}$ of memory and running Linux. Four were dedicated to readout of one quadrant of the TPC, while the remaining two read out RICH VME electronics and all CAMAC modules through two CBD 8210 CAMAC branch drivers. Each had a VME Interrupt Generator (VirGen) board for handling VME interrupts. These boards were designed at FNAL and built at Harvard University.

A dedicated I/O library was written to store data in a structured bytepacking format organized into blocks. A raw data file consisting of a file header block followed by any number of event blocks and an end of file block. Event blocks contained detector blocks consisted of data as it was read from VME or CAMAC with no pre-processing.

All processes were controlled from the E907 DAQ PC by a run control daemon. It maintained the overall state of the DAQ system, processed operator requests to start and stop data acquisition, and responded to exceptions 
and errors in the system. Communication to and from run control was handled by a message passing system implemented through stream sockets. Run configuration information was written to a Postgres database via a logger interface running on the DAQ server.

The event builder was supplied by FNAL Computing Division. Upon receiving subevents for each detector and each DAQ daemon, it combined them according to trigger time stamps before writing to disk. The event builder also divided long runs into subruns of $1 \mathrm{~GB}$ or less to facilitate subsequent file handling on the batch farm.

Online monitoring ran on a dedicated computer with data disks mounted through NFS. It scanned disks for new files, continuously updating histograms which helped shift workers identify any problems with the data. Online monitoring software was written in ROOT, and histograms for each run were saved for subsequent comparisons.

\subsection{Slow Controls}

Device settings and read backs as well as environmental parameters in the experiment hall such as temperature and pressure were controlled and monitored by computer. 


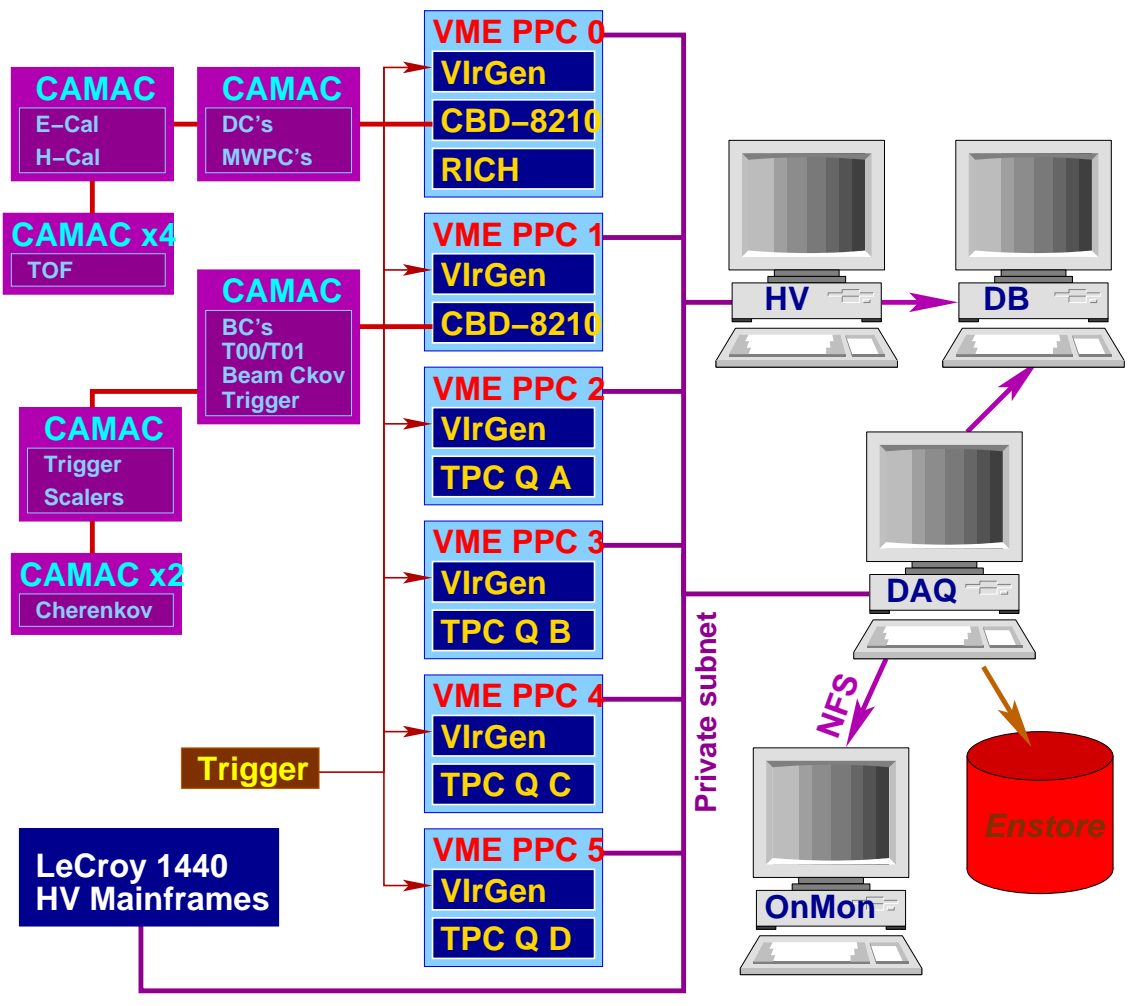

Figure 3.3: Schematic of the data acquisition system. 


\subsubsection{Monitoring Systems}

MIPP used two different systems to monitor and control most devices. The first was the FNAL APACS system using Itellusion iFix as a front end. It was used to:

1. Read air and gas pressures, temperature and humidity sensors

2. Control beam Cherenkov pressures

3. Control threshold Cherenkov counter gas flow

4. Act as an interlock for RICH cooling system

5. Monitor chamber gas flow and mixing

6. Monitor and control cryogenic target information

The second system was Accelerator Control NETwork (ACNET). It was developed at and is supported by FNAL. It was used for:

1. Controlling and monitoring secondary beamline quadrupole and dipole magnets

2. Controlling width of momentum collimator

3. Monitoring beam profiles

4. Monitoring spill structure

5. Monitoring spill intensity 
6. Controlling target wheel position

7. Controlling and monitoring analysis magnet currents

8. Monitoring analysis magnet Hall probes

9. Controlling beam Cherenkov mirror positions

10. Monitoring electromagnetic calorimeter and wire chamber voltages and currents

11. Controlling and monitoring RICH high voltage supplies

12. Monitoring hall and target temperature information

\subsubsection{Database Logging}

XML-RPC server provided by FNAL Beams Division was used for both control and and recording of values to the MIPP Postgres database for both iFix and ACNET devices. This was implemented via a Python script which queried all devices once every ten minutes and then logged values in the database.

The script also provided a GUI for shift workers which displayed all device names and the most recent read back values. Normally device names were displayed inside a green button. If the read back value was not within specified tolerances, the display turned red and an audible alarm was sounded. The alarm could be silenced by clicking on the button for the appropriate 
device. A status flag indicated whether or not a read back value fell within tolerances was included in the database log.

\subsubsection{High Voltage}

High voltage for TPC anodes and PMTs (excluding RICH) were controlled and monitored with two LeCroy 1440 systems. After values were set, the system continually cycled through all channels, storing read back voltages to the database and verifying that no trips had occurred. This was important for quickly catching TPC anode trips and minimizing data loss. 


\section{Chapter 4}

\section{MIPP Beam Line and Trigger}

\subsection{Beam Line}

The FNAL Main Injector facility (Figure 4.1) provided beam for the MIPP experiment. First, proton batches were accelerated by the LINAC and then the Booster, reaching $8 \mathrm{GeV} / c$. A maximum of 7 batches from the Booster could be simultaneously injected into the Main Injector, where they were then accelerated up to $120 \mathrm{GeV} / c$. Beam was extracted in a single turn from the Main Injector to the Switchyard, passing through section A of the Tevatron beam on the way. At this point it could be split up between the beam dump, Meson Test area, and Meson Center (MC), which included the MC7 hall in which MIPP was located.

Running conditions for MIPP were required to result in no more than a $5 \%$ slow down in $\bar{p}$ production for the Tevatron program. This meant that when 
MIPP started running, the final Booster batch was slowly extracted after the rest had been extracted for $\bar{p}$ production. Using this resonant extraction scheme MIPP initially received $600 \mathrm{~ms}$ spills at a rate of about 6 per minute. After the $\bar{p}$ stack size increased and the stacking rate decreased, MIPP was able to receive as many as 15 spills per minute. After the NuMI beamline began receiving protons for MINOS, the accelerator operating scheme was changed so that it could co-exist with $\bar{p}$ production. Single turn extraction was provided for each along with 4 second spills every 2 minutes for the Switchyard providing beam to MIPP. MIPP operated under these conditions from April 2005 to February 2006, collecting 36.3 million events.

\subsection{Secondary Beam}

The MIPP secondary beamline is shown in Figure 4.2.

Upon entering MC6, the proton beam from the Main Injector was focused onto a copper target. The hadrons produced by interactions in this target constituted the secondary beam. It was located about $97 \mathrm{~m}$ upstream of the secondary target and was a rectangular pipe with dimensions of $0.5 \times 0.5 \times$ $20 \mathrm{~cm}$. This is roughly 1.3 interaction lengths, so about $25 \%$ of the primary beam did not interact and was dumped into a series of concrete blocks.

Six quadrupole magnets were used to increase the dispersion of the secondary beam at the location of a pin-hole collimator, with three located before and three after. This allowed for reduction of the relative deviation 


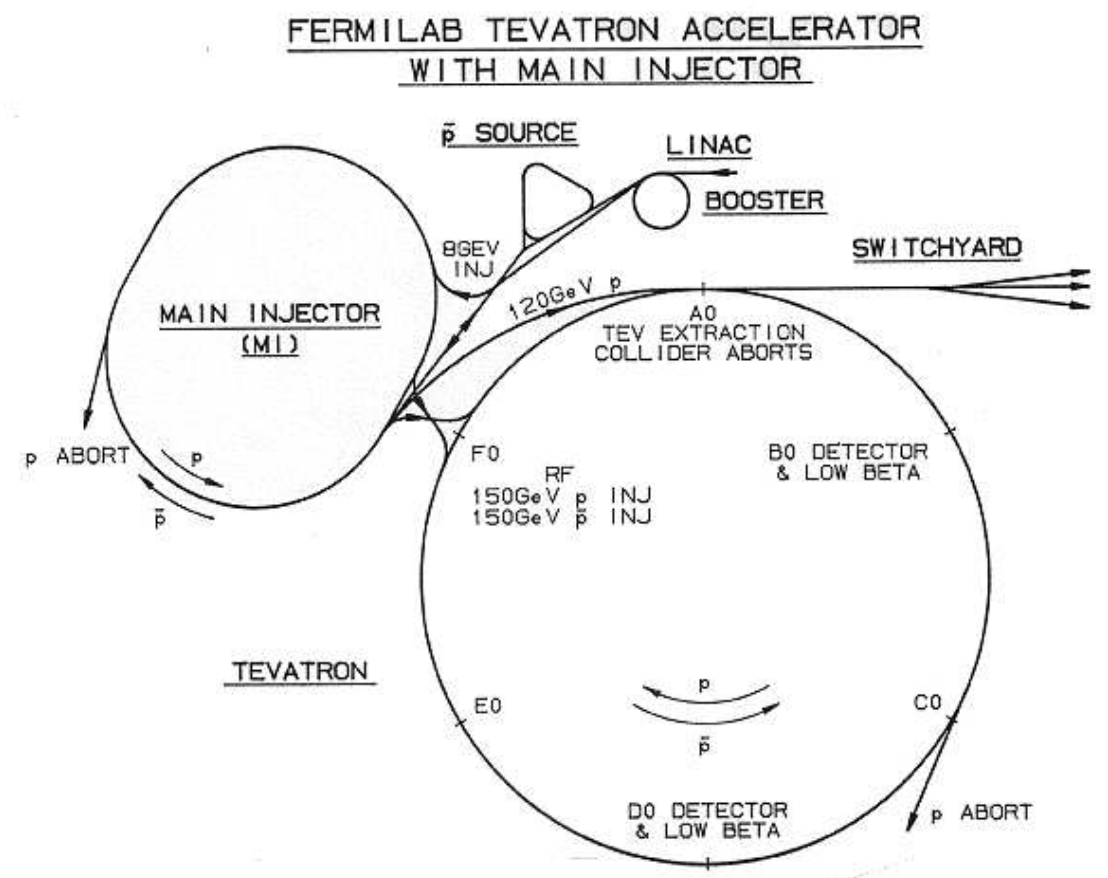

Figure 4.1: Fermilab accelerator complex. 
in momentum before focusing onto the experimental target.

\subsubsection{Beam Quality}

Simply having a well focused beam is not all that is required to take high quality data. This fact became quite clear once MIPP began taking data. An unacceptable amount of beam halo resulted in reduced data quality by causing the interaction trigger to fire erroneously and filling the TPC with so many tracks as to be unreconstructable. Although the interaction trigger and the TPC were not used during data taking for this analysis, improving the beam quality is still important since the excess beam halo can reach the RICH making it difficult to identify rings associated with incoming beam tracks.

The high amount of beam halo was being caused by scraping beamline elements. To correct the problem, a model of the secondary beamline was constructed using the software package OptiM [28]. Apertures of all beamline elements and magnetic field calculations based on measurements taken by FNAL Technical division were taken into account. Using this model, it was determined that scraping was occurring at several points, though most significantly at the last dipole magnet.

It was not possible to move beamline elements, so magnet currents had to be adjusted so that the beam remained focused and scraping was reduced. To do this, parameters were chosen at the target and the beam was transported backward and focused on the copper target while avoiding scraping 


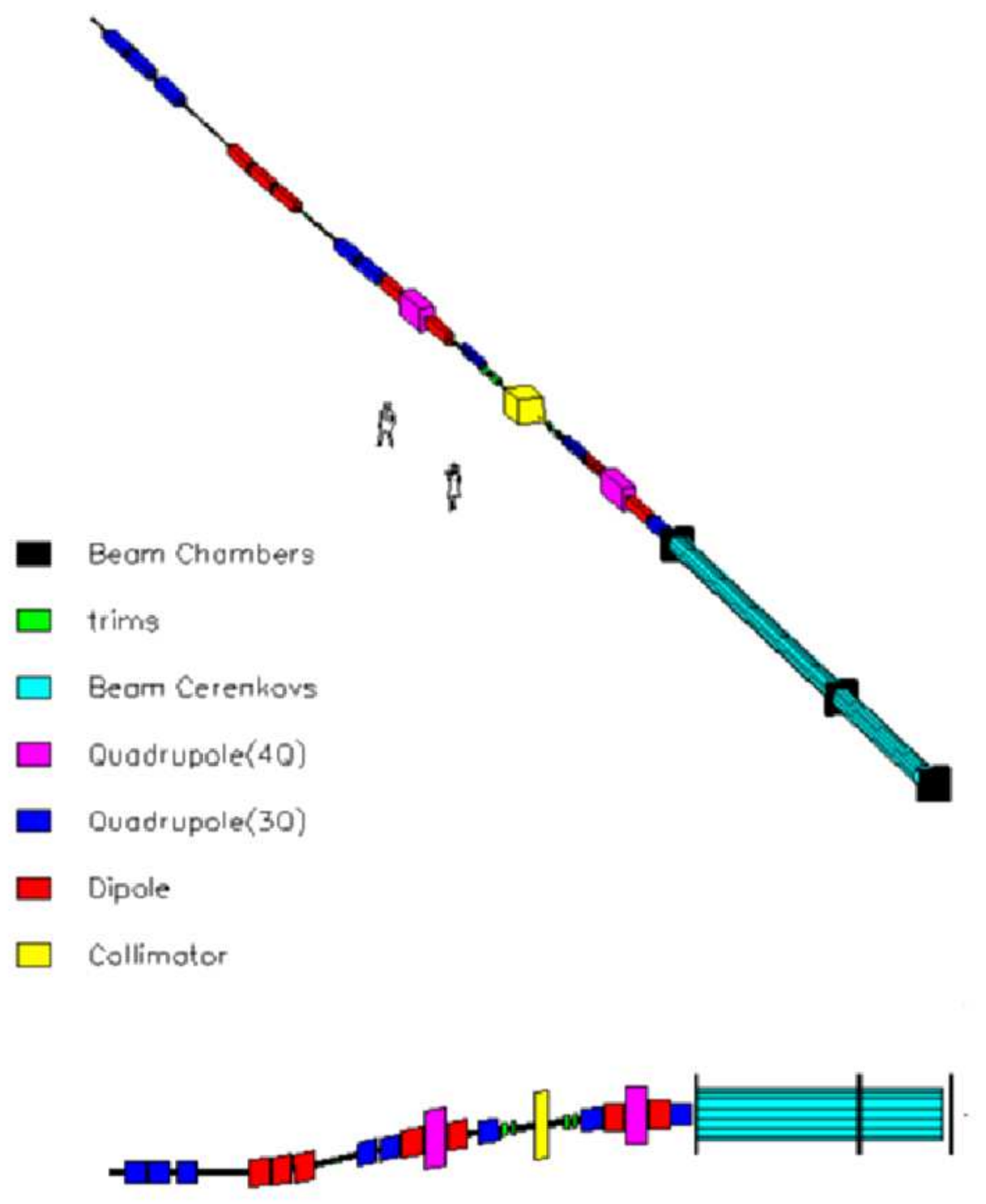

- Beom Chambers

$\square$ trims

Figure 4.2: MIPP beamline. 
of beamline elements. Using this method an acceptable solution was found. Tracking forward using this solution significantly reduced scraping and allowed more beam to make it through the collimator. Data quality clearly improved when this was implemented in the real beamline. See [27] for more details on this procedure.

\subsection{Trigger}

MIPP used a single-level experimental trigger in two stages: beam particle identification and determination of whether or not an interaction occurred. This means the seven trigger bits are needed: untagged beam, three particle species, and three particle species with interaction. Prescaling of trigger bits is applied before forming a global OR to achieve approximately $20 \%$ minimum bias and $80 \%$ interaction in normal running. These scaling factors were set to achieve equal amounts of pions, kaons, and protons.

When taking data for this analysis, the interaction trigger was not used and the target removed as only beam tracks are needed. As such, this section will only concern itself with the beam trigger and particle ID. Prescaling factors were chosen to result in an optimal mix of the three beam particle species. What this optimal mixture was and how it was determined is discussed in Chapter 6. 


\subsubsection{Beam Particle Identification}

In the momentum range of interest (above $35 \mathrm{GeV} / c$ ), it is possible to set the gas pressure in the Beam Cherenkov counters so that all beam particles produce light. They are identified based on which of the two PMTs in each counter saw light. They are designated as $U I$ for upstream inner, $U O$ for upstream outer, $D I$ for downstream inner and $D O$ for downstream outer. The particle trigger bits are then defined as:

- $\pi: \overline{U I} \cdot U O$

- $K: U I \cdot \overline{U O}$

- $p: D I \cdot \overline{D O}$

\subsubsection{Beam Cherenkov}

Optimal gas density for each counter was found by scanning over pressure and reading out PMT signals. 10000 triggers were taken at each pressure point. Given that there are four phototubes which can each be either on or off, there are 16 total combinations. By plotting the frequency at which each of these combinations occurs, it is possible to identify peaks corresponding to each of the beam species. This must be done each time beam momentum is changed. Figure 4.3 summarizes these pressure curves.

Figure 4.4 shows trigger purity as measured using RICH rings. Above 35 $\mathrm{GeV} / c$ all three species radiate and can be identified by fitted ring radius. 


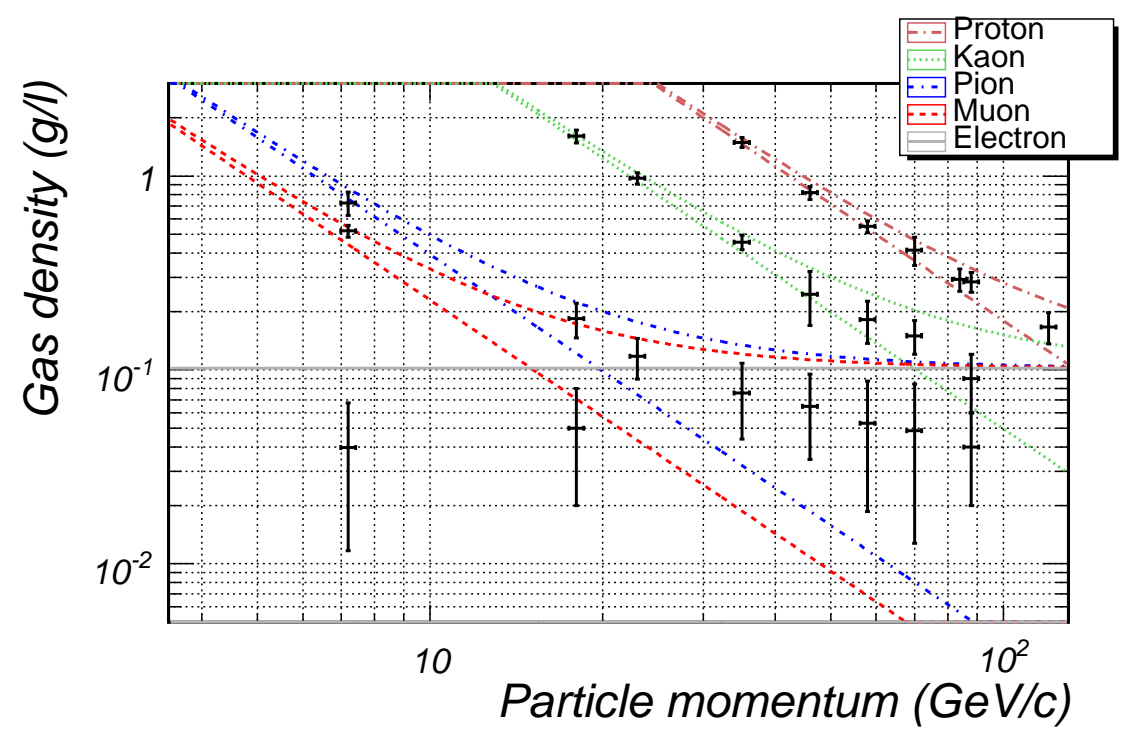

Figure 4.3: Upstream beam Cherenkov pressure curve summary. Two curves are displayed for each particle: threshold density and density when Cherenkov angle is $5 \mathrm{mrad}$. Data points are centers and widths of $U I \cdot \overline{U O}$ peaks in pressure curves.

For the momenta of interest, beam Cherenkov purity is above $80 \%$ and in most cases is well above 90\%. This is not significant for this analysis since the RICH itself provides much better separation.

\subsubsection{End of Spill and Calibration Triggers}

The end of each beam spill was followed by an end of spill trigger and then several calibration triggers. Rather than trigger the detector, the end of spill trigger was used to: readout scalers and clear them, record the number of different triggers in the spill to the database, and transfer buffered data from PPCs to the DAQ server. For the calibration triggers, a pulse generator 


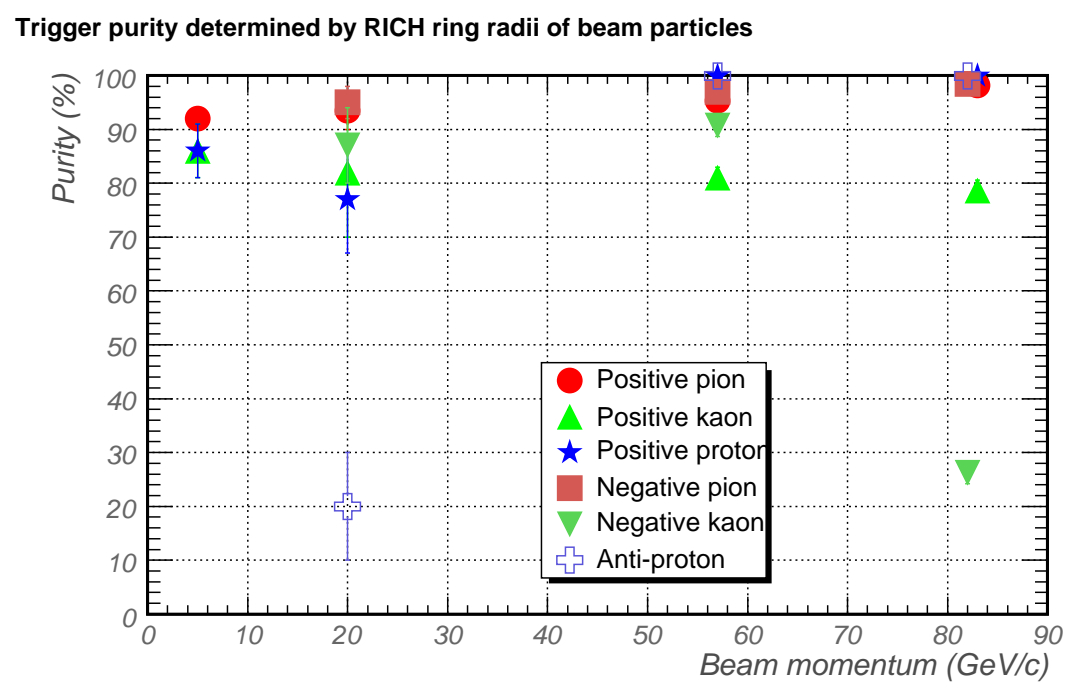

Figure 4.4: Beam trigger purity measured with the RICH.

was used to trigger the detector for the purpose of calculating pedestals and identifying hot channels.

\subsubsection{Veto Counter}

The veto counter was a scintillator paddle $35 \mathrm{~cm} \times 40 \mathrm{~cm}$ mounted $140 \mathrm{~cm}$ upstream of the experimental target on an aluminum plate. It had a 4.32 $\mathrm{cm}$ diameter hole in the center and two PMTs attached to sides using light guides. The logical AND of the two PMTs formed an 80 ns veto signal which was used to reject events with tracks outside the hole. This helped cut down further on beam halo. 


\section{Chapter 5}

\section{RICH Detector}

The MIPP RICH was built by the SELEX Collaboration [10] for use in that experiment. The following section summarizes the principle of operation of RICH counters, and the description and construction of the MIPP RICH as described in $[16,15,14]$.

\subsection{The Cherenkov Effect}

RICH counters exploit the Cherenkov effect [39, 22] for PID. This phenomenon occurs when a charged particle enters a medium traveling faster than the speed of light in that medium. For a qualitative description, first consider a charged particle moving slowly through some transparent medium such as a gas. The medium becomes polarized about the location of the particle due to its electric field. The shape of the atoms are distorted and 
they can be treated as elementary dipoles. Each point along the trajectory, then, will see a brief electromagnetic pulse as the particle passes through that point. However, there is no net field at large distances due the symmetry of the polarization. Therefore there is no radiation emitted.

Next consider a charged particle moving close to the speed of light in the medium. Now the polarization is no longer symmetric about the particle trajectory, though symmetry is maintained in the azimuthal plane. There is then a net dipole field even at distances far from the particle trajectory. A brief electromagnetic pulse is then radiated from each point as the particle passes through.

Generally, wavelets radiated from all points along the trajectory interfere destructively, which means that the net field even at large distances is again zero. The wavelets can be in phase, though, if the particle velocity exceeds that of light in the medium. The net field results in emission of radiation at a certain angle $\theta$ with respect to the trajectory, as can be seen from Huygens principle. Consider two points A and B along the trajectory and a third point C away from the trajectory such that the points form a right triangle and the angle between $\mathrm{AC}$ and $\mathrm{AB}$ is $\theta$ (See Figure 5.1). Wavelets from points along $\mathrm{AB}$ are coherent and form a plane wave when the particle travels $\mathrm{AB}$ in the same time the light travels $\mathrm{AC}$. The speed of the particle is $\beta \mathrm{c}$ where $\mathrm{c}$ is the vacuum speed of light. The particle travels a distance $\mathrm{AB}=\beta c \Delta t$ in time $\Delta t$. In the same time, the light travels $\mathrm{AC}=\Delta t(c / n)$ where $n$ is the refractive index. The cosine of the angle is $\mathrm{AC} / \mathrm{AB}$, with the result that: 


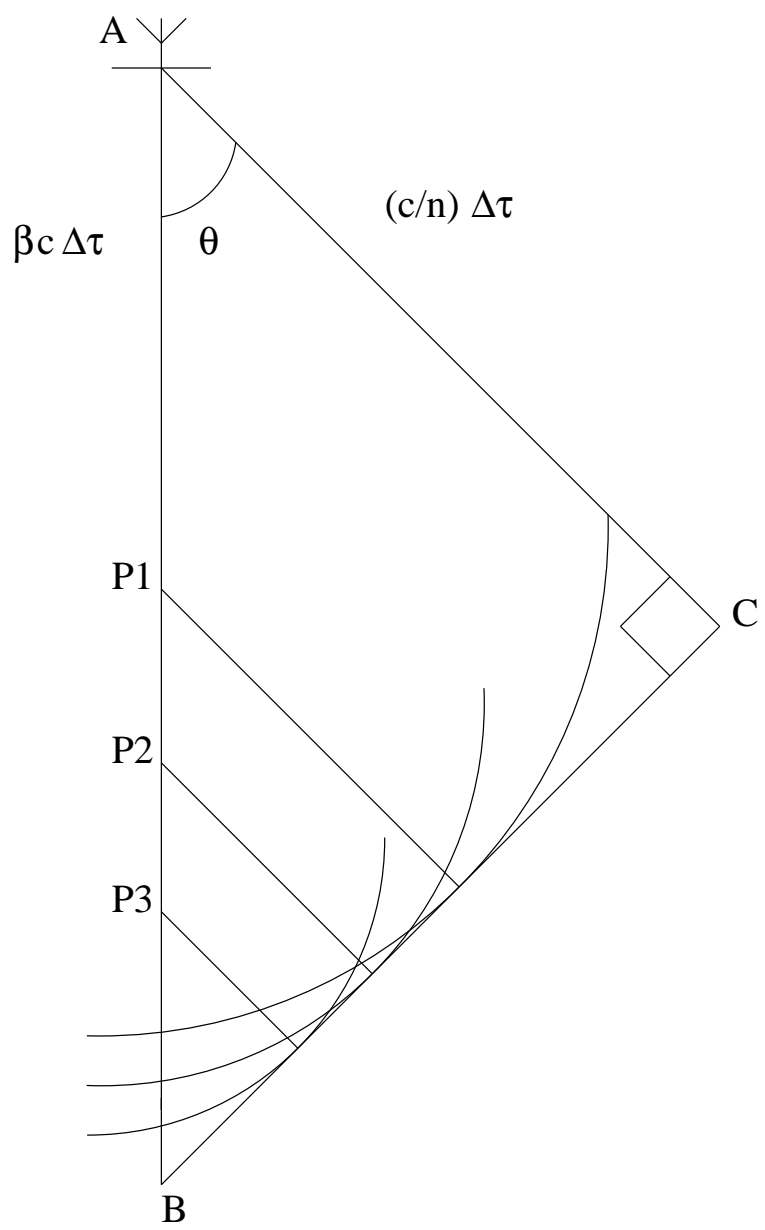

Figure 5.1: Diagram of the Cherenkov effect. A charged particle traveling faster than the speed of light in medium creates an asymmetric polarization. This results in a brief electromagnetic pulse forming a coherent wavefront at angle $\theta$. 


$$
\cos \theta=\frac{1}{\beta n} .
$$

This relation shows some characteristics of Cherenkov radiation. For a given index of refraction $n$, no light is emitted below a threshold velocity $\beta_{t h r}=1 / n$. There is a maximum angle at which light can be emitted, corresponding to $\beta=1$ : $\theta_{\max }=\cos ^{-1}(1 / n)$. Cherenkov light is mainly in the visible and ultraviolet regions and is emitted along the surface of a cone defined by the angle $\theta$. The number of photons emitted per unit radiator length over the wavelengths $\lambda$ to $\lambda+d \lambda$ is given by the formula

$$
\frac{\partial^{2} N_{p h}}{\partial \lambda \partial x}=\frac{2 \pi \alpha}{\lambda^{2}} \sin ^{2} \theta
$$

where $\alpha$ is the fine structure constant.

\subsection{Physical Description}

The MIPP RICH detector consists of a low carbon cylindrical steel vessel $10.22 \mathrm{~m}$ in length, 93 in in diameter with a wall thickness of $\frac{1}{2}$ in. The ends are sealed with 1.5 in thick aluminum with cutouts for thin beam windows at each end and a photomultiplier tube holder plate. A schematic is shown in Figure 5.2.

The vessel has several piping connections for delivery of gas to fill the detector volume. The index of refraction of the radiator is important to 


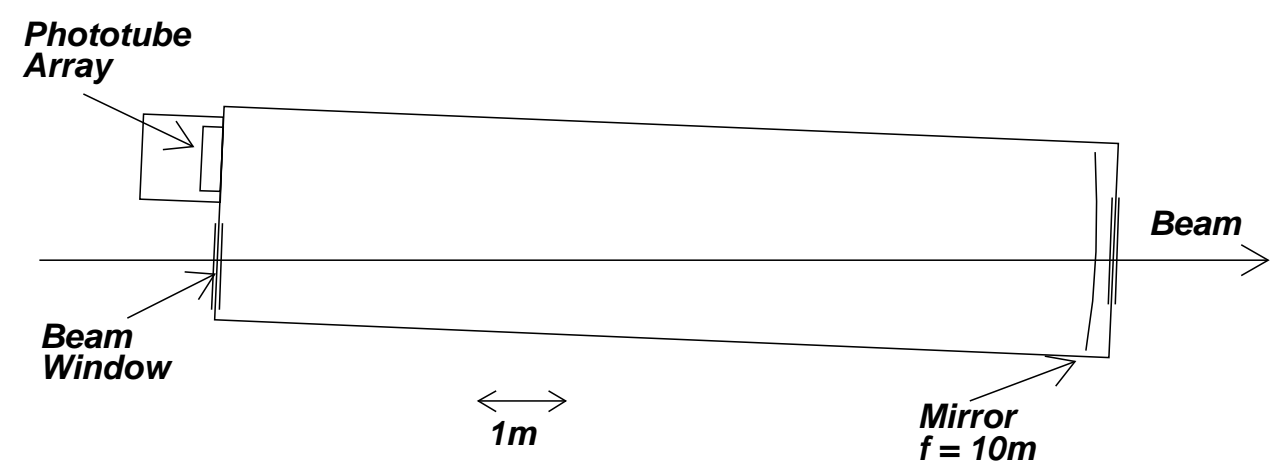

Figure 5.2: Schematic of RICH detector from [16].

consider as it determines momentum thresholds and ring sizes. MIPP used $\mathrm{CO}_{2}$ at just above atmospheric pressure as the radiator. The refractive index at STP is about 1.00045. With this configuration thresholds were $4.5 \mathrm{GeV} / c$ for pions, $17 \mathrm{GeV} / c$ for kaons, and $31 \mathrm{GeV} / c$ for protons with a $\beta=1$ ring radius near $29.5 \mathrm{~cm}$. The RICH provides $3 \sigma \pi / K$ up to $80 \mathrm{GeV} / c$ and $3 \sigma$ $p / K$ separation up to $120 \mathrm{GeV} / c$.

There are also electrical feedthroughs for gas-tight vacuum coaxial connectors, which are used for monitoring of the internal vessel temperature pressure, and an LED array for calibration.

In order to reduce photon reflections inside the vessel, a coating of black paint was applied. A low out-gassing paint was chosen to prevent contamination of the radiator gas. The inner surface was sand-blasted before painting and then sprayed.

The vessel is covered by $15 \mathrm{~cm}$ thick building insulation. A water coil, which is wrapped around the vessel and lies beneath the insulation, in con- 
junction with a chilled water system helped regulate the temperature.

\subsubsection{Entrance and Exit Windows}

Both the entrance and exit windows consist of three layers: 3 mil Kevlar

cloth, 1 mil aluminum foil and 1.5 mil charcoal Tedlar. Each window was inserted first between two mylar rings, then between two aluminum rings which were bolted together along with a rubber o-ring seal and mounted on the vessel. Permeability was checked with both air and neon gas, while light tightness was checked by observing the noise rate for a phototube placed inside a box which had one side made of the same material as the windows. Both tests showed that the window material is both gas and light tight [16].

\subsubsection{Mirrors}

A mirror plane is mounted at the downstream end of the RICH, focusing Cherenkov light onto the phototube array. It covers a total area of $2.4 \mathrm{~m} \times$ $1.2 \mathrm{~m}$ and consists of 16 spherical, hexagonally shaped mirrors arranged in three rows. There are six mirrors in the central row and five each in the top and bottom rows as shown in Figure 5.3.

They are made from low expansion glass, measure $40 \mathrm{~cm}$ across, $46 \mathrm{~cm}$ tip to tip, and are $10 \mathrm{~mm}$ thick. The mirrors on each end of the central row were truncated by 3 in to fit inside the vessel. Of the two central mirrors, the rightmost one has a $6.8 \mathrm{~cm} \times 11 \mathrm{~cm}$ section removed which allows beam 


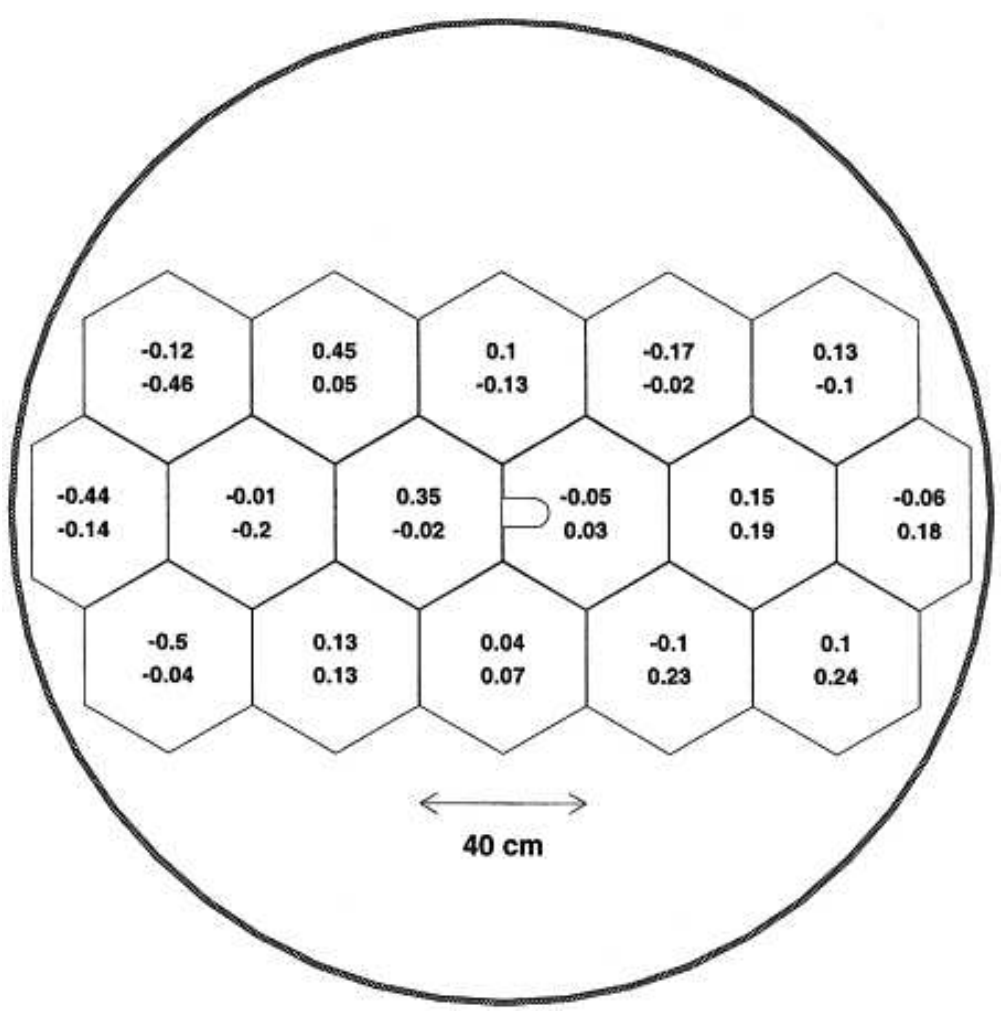

Figure 5.3: Drawing of RICH mirror layout from [16]. The top number shown in each mirror is the deviation from the average center of curvature in $\mathrm{cm}$ for the horizontal coordinate. The lower number is for the vertical coordinate.

particles to pass through without interacting in the mirror. The average mirror radius is $1980 \mathrm{~cm}$ with a deviation of less than $5 \mathrm{~cm}$ not only within one mirror but also between each mirror.

Each mirror was polished such that an image size of a point source illuminating the mirror was less than $1 \mathrm{~mm}$. An aluminum coating and an overcoating of $\mathrm{MgF}_{2}$ were applied to achieve high reflectivity, greater than $85 \%$ at $160 \mathrm{~nm}$. 
Mirror sphericity was measured using the Ronchi method [36]. A light source is placed near the center of curvature with a narrow slit forming a line source. A fine grating, called a Ronchi Ruling, is inserted in the path of reflected light at a known distance. This causes alternating light and dark bands to appear across the mirror. By counting the number of these bands, the image distance and hence the radius of curvature can be found The mirrors were arranged so that the best sphericity was in the center of the array, and to minimize differences in average radius between neighboring mirrors.

Three point kinematic mounts are used to affix each mirror individually to a flat, low mass, lin thick honeycomb panel. These mounts consist of an aluminum pad glued to the back of a mirror, a ball bearing, a $\frac{1}{4}$ in diameter double differential screw made of titanium with a thread of $80 /$ in, a $\frac{1}{2}$ in aluminum cylinder with a thread of 13/in and a 1in diameter nylon cylinder. This allows the mirrors to be aligned on a sphere with an angular accuracy of $50 \mu \mathrm{rad}[16]$.

\subsubsection{Phototubes}

Phototubes used are of two different types of $\frac{1}{2}$ in diameter, $10 \mathrm{~mm}$ bi-alkali photocathode, 10-stage tubes: Hamamatsu R760 and Russian made FEU60. The R760 tubes have a quartz entrance window and response down to 170 nm. The FEU60 tubes have a glass entrance window and are coated with a wavelength shifter so as to achieve sensitivity over the same range of wave- 
lengths as the R760 tubes. Thickness of the wavelength shifter was found not to be critical to performance, based on tests made in a vacuum reflectometer using a Deuterium light source [16].

The two types of phototubes differ significantly in terms of quantum efficiency and noise. The R760 tubes have a quantum efficiency of about $25 \%$ at a peak wavelength of $350 \mathrm{~nm}$. The FEU60 tubes are an average less than half as as efficient, with a peak efficiency of $11 \%$. They also have an order of magnitude greater noise than the R760 tubes. Quantum efficiencies were measured, as described in [16], relative to a standard R760 tube, of which the absolute quantum efficiency was known only approximately. This was done inside a dark box using blue LED light delivered to 32 identical tube holders and the standard tube via optical fibers. The LED delivered on the order of $5 \%$ of a photoelectron per tube per pulse. The output of each tube was digitized and sent to two scalers: one gated for 100 ns in time with the LED, and one for the same time midway between pulses. Noise level from the second scaler was subtracted from the first to arrive at the correct phototube response to the LED pulses. A third scalar was used to count the number of pulses for normalization purposes. High voltage was supplied by a CAMAC controlled supply, and was scanned over the rang of operating voltages. Approximately $5 \times 10^{5}$ pulses were taken at each high voltage point.

Using data from all phototubes, similar groups of 32 tubes were identified with a single value for operating voltage. Each group consisted of 
a single type of tube, as operating voltages for R760 tubes are lower than that of FEU60 tubes. Voltages were selected where the quantum efficiency plateaued, or where the noise reached $30 \mathrm{kHz}$. Columns were alternated by type for the central region of the array, with only FEU60 tubes using in the outer portions. In total, 19 of the 89 columns were R760.

\subsubsection{Holder Plate}

The aluminum holder plate, shown in Figure 5.4, which houses the phototubes is 55in wide, $27 \mathrm{in}$ high and 3in thick. An array of 2848 holes have been drilled through the plate to hold the phototubes. They are packed hexagonally in 89 columns of 32 each with a spacing of 0.635 in, and have a diameter of 0.6 in

Each hole is, on one side, a straight channel 2 in in depth which houses a phototube. A $2 \mathrm{~mm}$ thick quartz window is glued in place between the tube and the other side of the hole. It provides a gas seal between the phototube and the carbon dioxide radiator gas. Gas tightness was checked with a helium leak detector, and any windows found to leak were replaced [16]. Any residual glue was cleaned off each window, and transmission was checked with a spectrometer [16]. It was found to be greater than $90 \%$ at wavelengths above $200 \mathrm{~nm}$.

The other side of each hole is a 1 in deep, tapered channel of inner radius 0.4 in. This size was chosen to match that of the photocathode of the phototubes. Aluminized mylar cones are inserted into each hole on this side, 

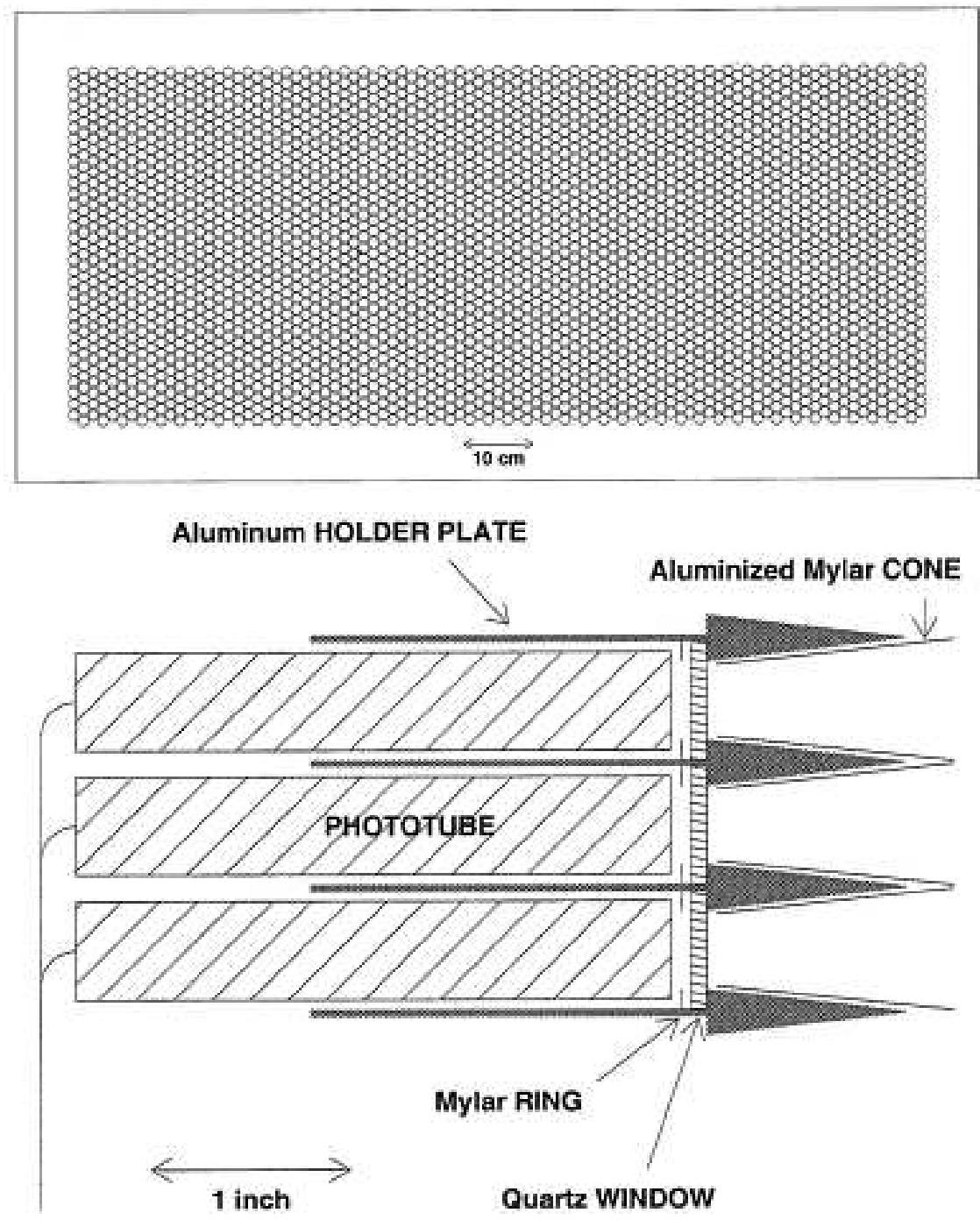

Figure 5.4: Drawing of RICH holder plate from [16]. Top: Front view. Bottom: Partial cross section. 
extending slightly from the block. They allow for nearly $100 \%$ coverage for photon detection. Reflectivity of the cones was measured and found to be greater than $80 \%$ for wavelengths above $300 \mathrm{~nm}$.

To ensure that each tube is held in place and centered on the aperture, Velcro strips were fastened along the length of the tube. A 3 mil mylar ring of inner radius 0.4 in was placed against each window to keep the gap between phototube and window as small as possible. This minimized loss of UV photons and prevented removal of PTP wavelength shifter coating which was applied to FEU60 phototubes.

\subsubsection{High Voltage}

Operating voltages for FEU60 tubes ranged from $1300 \mathrm{~V}$ to $1900 \mathrm{~V}$ with a current draw of $150 \mu \mathrm{A}$ per tube, and $900 \mathrm{~V}$ to $1250 \mathrm{~V}$ with a current draw of $300 \mu \mathrm{A}$ for R760 tubes. In order to deliver high voltages over this large range, six air cooled zener diode chains each with its own Glassman EK3R200 HV power supply were used. Four of the supplies powered FEU60 tubes and were set to trip if the current exceeded $100 \mathrm{~mA}$. The other two powered R760 tubes and were set to trip at $140 \mathrm{~mA}$.

The voltage for each column was selected by soldering a high voltage cable directly to the proper location on the chain. This distribution system is contained within an aluminum box mounted to the side of the phototube box. As originally constructed, high voltage cables were soldered together with the wires to each of the 32 tubes in a bundle to a small fanout board. 
The ground returns were also soldered to fanout boards and connected to a common feedthrough panel. A support system was put in place to hold the fanout boards. This meant that high voltage cables had to be unsoldered from the fanout boards each time a PMT bundle needed to be removed and then re-soldered when the bundle was put back in place.

To prevent damage to the phototubes, an interlock system was implemented to turn off high voltage if the phototube box cover is removed. At each corner of the box is installed one of four rocker switches which will open the high voltage circuit if the cover is not firmly closed. Also included as part of the interlock system are three Klixon temperature sensors inside the box which will open the circuit if the temperature exceeds 50 degrees Celsius. This offers protection in the event of a cooling system failure.

The cooling system consists of a water chiller and a light tight heat exchanger. Cooled air is delivered to the phototube box via ducts and enters through a long slit at the bottom of the box near the tubes. Several fans mounted to the inside of the cover improve circulation, and there are two air returns located near the top, one on each side of the box. Heat dissipated inside the PMT box is estimated to be about 900 Watts, entirely in the resistor chain providing high voltage to the PMT dynodes.

\subsubsection{Readout System}

Each column of 32 phototubes is divided into two bundles of 16 . Wires carrying output signals from each group are soldered onto paddles cards, 
which plug into a light tight backplane of one of three custom made crates on top of the phototube box.

The paddles have $1 \mathrm{k} \Omega$ resistors for each channel to protect the readout chips from charge buildup when they are connected. The backplane uses

96-pin Eurocard connectors. Only every third column is used, alternating between signal and ground. This helps prevent cross talk between signals. All unused pins are grounded.

Readout electronics are mounted on cards which are installed in the crates. The original cards used in SELEX were replaced with new custom electronics designed at FNAL and built and tested at Harvard University. The $\pm 6 \mathrm{~V}$ operating voltage is supplied along the backplane by high current connectors. Cooling is supplied by fans mounted on each crate and is monitored by air flow sensors. A schematic of this setup is shown in Figure 5.5 .

\subsubsection{LED Monitoring System}

Four chains of 10 blue LEDs each are mounted to the mirror support frame at the downstream end of the vessel. Only two of these chains are actually used, with the other two as spares. Each LED has a separate driver circuit, ensuring short light pulses, fed from a 12 VDC power supply and a pulse generator. The amount of light is controlled by adjusting the pulse amplitude on the generator. Due to the large distance from the phototube array, light is distributed evenly and the short pulses produce the same PMT response 


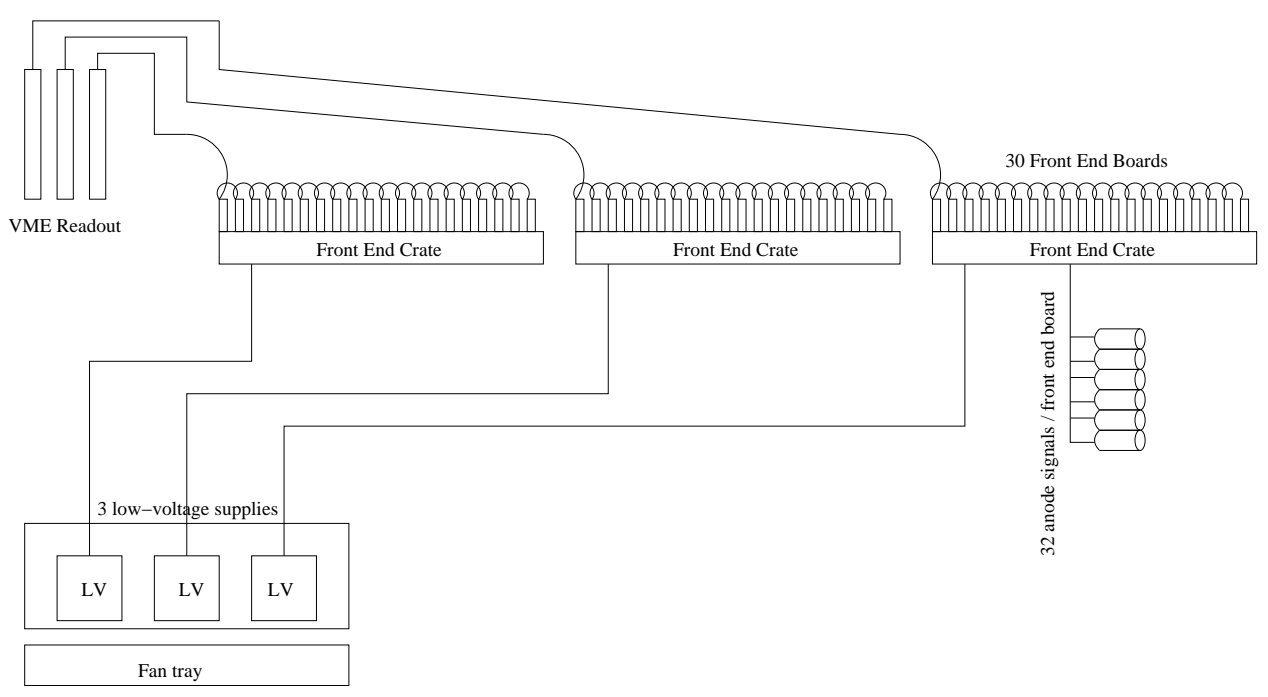

Figure 5.5: Schematic of RICH electronics.

as Cherenkov light. This allowed for calibration of the tubes using the LEDs.

\subsection{The RICH in MIPP}

The RICH vessel was mounted on a rail system which allowed it to be moved back if need be, such as for repair of multi-wire proportional chambers which were installed directly upstream and downstream of the RICH. Stops on the rails allowed the vessel to be re-positioned within $1 \mathrm{~mm}$ after being moved. The orientation of the vessel was 2.4 degrees off the horizontal axis.

Before running began, MIPP revised the operating voltages for each column. The grouping of phototubes into bundles as determined by SELEX was not changed. This was done with each column installed in the RICH and used the built in LED. Each column was scanned over the range of operating 
voltages and was readout in time with LED flashes and between flashes to determine the background rate. Operating values were chosen to optimize quantum efficiency vs. noise rate.

\subsubsection{RICH Fire}

During commissioning of the experiment smoke alarms were registered on top of the RICH and at nearby relay racks. The smoke detectors had shut down power for the downstream part of the experiment as they had been intended to do. Upon investigation smoke was observed near the RICH electronics. The readout cards were tested and were working properly, which suggested the problem was inside the PMT box. After opening the box, cables and insulating wrapping were found melted in a region on the right side of the array. A panel was formed to investigate the incident. Their findings are documented in [4].

The melting point of the insulation wrapped around the PMT bundle wires was measured to be $266 \mathrm{C}$. The temperature Klixons should have cut off high voltage once the temperature exceeded $50 \mathrm{C}$, and in fact the $\mathrm{HV}$ interlock had been tripped. The Klixon near the affected region had been burned and was no longer usable. The other two were tested using an oven which allowed temperature to be raised gradually to $80 \mathrm{C}$. Resistance across the leads was measured with an ohmmeter. Both were found to be working properly by opening when the temperature exceed $50 \mathrm{C}$ [34].

When these cables were removed from the affected region a large number 
of PMT bases were found melted and fused together. The evidence strongly suggested that gradual heating was not the problem, rather that a fire had occurred.

Bases were tested for flammability [33]. Pairs of bases, one FEU60 and one R760, were subjected to a series of three tests. The first applied a flame to the bases, the second used a piezo-electric spark generator, and for the third only an R760 base was tested. Conclusions drawn were that enough energy was present to ignite the FEU60 base and have self sustaining burning without any additional energy. FEU60 bases were consumed in 2 to 3 minutes, dripping burning material which spread the fire. There was also enough flame energy to spread the fire to adjacent tubes horizontally and vertically. The R760 bases did not show evidence of self sustained burning and likely helped limit the fire along with the backward airflow. The duration of the fire was likely between 3 to 10 minutes.

From a visual examination the fire was most intense in column 73 near the bottom of the array, likely the point of origin of the fire. Column 73 was an FEU60 column.

The cause was likely shorting in the FEU60 tube bases [26], which were not as well constructed as the R760 bases. In many cases exposed wire ends were nearly touching each other or solder joints at points along the resistor chain which led to sparking. Sparking was directly observed when $1500 \mathrm{~V}$ was applied to a single resistor in one of the bases to test this possibility. The excess current from a spark was about $2 \mathrm{~mA}$. This was not enough to 
trip the power supply, so repeated sparking could have occurred, possibly causing the flammable base covering to ignite since it was in direct contact with the resistor chain.

Refurbishing the RICH and implementing fire prevention and safety measures was a long and involved process. First, the undamaged phototubes were removed and stored. Removing tubes in the area of the fire took much longer as many of the bases had fused together. The vessel was then moved from the experiment hall to Lab 5 at FNAL.

Residue from the fire needed to be cleaned out of the light box and removed from the quartz window using q-tips. Using a column at the opposite end from the area affected by the fire as a baseline, the transmission of the quartz window for each column was measured. Results were consistent with the unaffected column assuring that transmission was not compromised.

About $25 \%$ of all phototubes were lost due to destroyed bases. All of the surviving bundles were tested one at a time in a dark box with an LED. The tubes were mounted in small, rectangular aluminum block with holes drilled in four columns for four rows. Optical fibers were used to distributed light evenly to each of the tubes which were readout with an Analog to Digital Converter (ADC). There were some dead tubes found though most were in good working order.

Tubes from partially destroyed bundles were combined together to from complete bundles, or used to swap out dead tubes from surviving bundles. There were not enough available tubes to replace all of the dead ones. Volt- 
ages were matched as closely as possible when combining tubes from different bundles. The newly formed bundles were tested in the dark box.

To help prevent the FEU60 bases from catching fire again, the exposed wire ends were pulled away from each other and solder points. The bases were cleaned and sprayed with an insulating acrylic conformal coating. The old flammable base coverings and bundle wrapping were replace with a nonflammable shrink wrapping applied using a heat gun. It was noticed that the wavelength shifting coating had begun to wear off some of the FEU60 tubes, so it was reapplied to those tubes.

When the RICH was moved back into the experiment hall and reassembled, the high voltage delivery system inside the PMT box was redone. Short HV cables were soldered to the fanout for each bundle with an SHV connector on the other end. The fanouts were covered with the same shrink wrapping used on the bases. SHV connectors were attached to the ends of the cables running from the zener chain to the inside of the box and the support for the fanouts removed. This made removal and installation of bundles easier as soldering was no longer needed. The Klixons were put back into the interlock with the burned one replaced.

We were left with enough tubes for 68 complete columns out of the original 89, with 15 R760 and 53 FEU60. The were arranged symmetrically about column 45 , the center of the array and packed more densely near the center. Every fourth column up through 40 was left empty, as was every fourth column starting with 50. All columns between 40 and 50 were filled. The 
R760 tubes were placed in every fourth column starting with 17 and ending with 73 . The only exceptions to this symmetric arranged were the top half of column 7 and the bottom half of column 89, which were left missing.

Once all columns were installed the operating voltages were again determined using the built in LED as previously described. This had to be done a few columns at a time as clearance to run the RICH fully turned on had not yet been obtained.

Several safety precautions needed to be put in place before clearance was granted. A Nuclear Instrument Module (NIM) circuit was designed and built at Indiana University to trip an HV power supply when a current surge is detected due sparking. The PMT box was inerted with nitrogen and oxygen content was reported to APACS. Temperature monitors and a Very Early Smoke Detection and Alarm (VESDA) system were installed in the PMT box and also connected to APACS for monitoring. APACS would then cut off high voltage if any of these systems produced an alarm.

\subsubsection{RICH Slow Controls}

In addition to fire safety parameters, the following status parameters were monitored and logged in a database once every ten minutes throughout the run: atmospheric pressure, vessel pressure, vessel temperature (at both upstream and downstream ends), and voltage and current draw on all six high voltage power supplies.

From temperature and pressure readings we were able to track the density 
of $\mathrm{CO}_{2}$ inside the RICH. A leak was discovered on the order of $10 \mathrm{~atm} \mathrm{cc} / \mathrm{s}$ and the vessel needed to be topped off once every two weeks. This is in contrast to SELEX, which leaked checked the vessel using a helium mass spectrometer and found it to leak no more than $10^{-9} \mathrm{~atm} \mathrm{cc} / \mathrm{s}$ [16]. 


\section{Chapter 6}

\section{RICH Mass Measurement Concept}

This chapter deals with the basic theory of RICH detectors as related to this analysis. The principles of RICH counters are covered in [38, 35, 31].

\subsection{Role of RICH Detector}

The Ring Imaging Cherenkov Detector (RICH) uses spherical mirrors to focus Cherenkov light emitted as a charged particle travels through the radiator volume onto an array of photomultiplier tubes situated at the focal plane. The light, which is emitted as a cone, forms a ring when it reaches the PMT array. From the pattern of hit PMTs, this ring can then be reconstructed. Ring radius provides a measurement of the mass of a particle with known 
momentum, and hence provides particle identification.

\subsubsection{Basic Mass Measurement Concept}

This discussion follows from [24].

From the expression for the Cherenkov angle, Eqn. 5.1, and the geometry of the RICH, the radius of a ring can be related to $n$, the refractive index of the radiator gas, and $\beta$ of the particle:

$$
R=F_{L} \sin ^{2} \theta=F_{L} \sqrt{n^{2}(\lambda) \beta^{2}-1},
$$

where $F_{L}$ is the focal length of the mirror. Thus the reconstructed ring radius provides a measure of the Cherenkov angle.

Due to dispersion in the radiator, a ring has some thickness and cannot be fully described by a single radius. For simplicity, consider only a single angle corresponding to the peak of the light distribution, which from 5.1 can approximated by

$$
\theta=\sqrt{2\left(1-\frac{1}{n \beta}\right)} .
$$

and then solving for the refractive index

$$
\frac{1}{n}=\beta\left(1-\frac{\theta^{2}}{2}\right) .
$$

Consider two particles $i$ and $j$ with momentum $p$ which have masses $m_{i}$, 
$m_{j}$ and emit Cherenkov light at angles $\theta_{i}, \theta_{j}$ respectively. One can then relate the two angles:

$$
\frac{\theta_{i}^{2}}{2}=1-\frac{\beta_{j}}{\beta_{i}}\left(1-\frac{\theta_{j}^{2}}{2}\right) \approx 1-\frac{\beta_{j}}{\beta_{i}}+\frac{\theta_{j}^{2}}{2} .
$$

In the relativistic limit,

$$
E=\sqrt{p^{2}+m^{2}}=p \sqrt{1+\frac{m^{2}}{p^{2}}} \approx p\left(1+\frac{m^{2}}{2 p^{2}}\right)
$$

and thus

$$
\beta=\frac{p}{E}=\frac{1}{1+\frac{m^{2}}{2 p^{2}}} \approx 1-\frac{m^{2}}{2 p^{2}} .
$$

Using this expression for $\beta$ we see that

$$
1-\frac{\beta_{j}}{\beta_{i}}=1-\frac{1-\frac{m_{j}^{2}}{2 p^{2}}}{1-\frac{m_{i}^{2}}{2 p^{2}}} \approx \frac{m_{j}^{2}-m_{i}^{2}}{2 p^{2}}
$$

which, when substituted into Eqn. 6.4 results in the expression

$$
\theta_{i}^{2}-\theta_{j}^{2}=\frac{m_{j}^{2}-m_{i}^{2}}{p^{2}}
$$

By relating pion rings separately to proton and kaon rings through Eqn. 6.8 we can express the kaon mass in terms of the masses of the other two particles and all three angles. 


$$
\begin{gathered}
\theta_{\pi}^{2}-\theta_{K}^{2}=\frac{m_{K}^{2}-m_{\pi}^{2}}{p^{2}} \\
\theta_{\pi}^{2}-\theta_{p}^{2}=\frac{m_{p}^{2}-m_{\pi}^{2}}{p^{2}}
\end{gathered}
$$

Solving for kaon mass

$$
m_{K}^{2}=m_{\pi}^{2}+\Delta_{p \pi} \frac{\theta_{\pi}^{2}-\theta_{K}^{2}}{\theta_{\pi}^{2}-\theta_{p}^{2}},
$$

where $\Delta_{p \pi} \equiv m_{p}^{2}-m_{\pi}^{2}$.

Making use of a mono-energetic beam of protons, kaons, and pions is therefore essential. The idea is to measure the three Cherenkov angles, which are related to each other and the masses of each particle as in Eqn. 6.11. The proton and pion masses are known to much higher precision than the kaon mass, and are the key to understanding systematic uncertainties.

\subsubsection{Estimated Uncertainty}

An estimate for the statistical uncertainty can now be made by evaluating partial derivatives [23]:

$$
\begin{gathered}
\frac{1}{2} \frac{\partial}{\partial \theta_{\pi}} m_{K}^{2}=\Delta_{p \pi} \theta_{\pi} \frac{\theta_{K}^{2}-\theta_{p}^{2}}{\left(\theta_{\pi}^{2}-\theta_{p}^{2}\right)^{2}}=p^{2} \theta_{\pi} \frac{\Delta_{p K}}{\Delta_{p \pi}} \\
\frac{1}{2} \frac{\partial}{\partial \theta_{K}} m_{K}^{2}=-\Delta_{p \pi} \frac{\theta_{K}}{\theta_{\pi}^{2}-\theta_{p}^{2}}=p^{2} \theta_{K}
\end{gathered}
$$




$$
\frac{1}{2} \frac{\partial}{\partial \theta p} m_{K}^{2}=\Delta_{p \pi} \theta_{p} \frac{\theta_{\pi}^{2}-\theta_{K}^{2}}{\left(\theta_{\pi}^{2}-\theta_{p}^{2}\right)^{2}}=p^{2} \theta_{p} \frac{\Delta_{K \pi}}{\Delta_{p \pi}} .
$$

The kaon mass uncertainty is then

$$
\frac{1}{4} \sigma_{m_{K}^{2}}^{2}=p^{4}\left[\theta_{\pi}^{2}\left(\frac{\Delta_{p K}}{\Delta_{p \pi}}\right)^{2} \sigma_{\theta_{\pi}}^{2}+\theta_{K}^{2} \sigma_{\theta_{K}}^{2}+\theta_{p}^{2}\left(\frac{\Delta_{K \pi}}{\Delta_{p \pi}}\right)^{2} \sigma_{\theta_{p}}^{2}\right]
$$

It is proportional to the square of momentum, which means it is best to make this measurement at as low a momentum as possible. Of course it is important to remain high enough above threshold, which for protons is approximately $31 \mathrm{GeV} / c$. Therefore it was decided to run at $40 \mathrm{GeV} / c$.

Determining the optimal fraction of pion, kaon, and proton triggers is also necessary. Using Eqn. (6.2) and assuming that the width of a ring is due to dispersion of $\mathrm{CO}_{2}$ and momentum bite of the beam, then the angular distribution of RICH rings for particle species $i$ has a width $w \theta_{i}$ of:

$$
w \theta_{i}^{2}=\left(\frac{1}{\theta_{i} n^{2} \beta_{i}}\right)^{2} \sigma_{n}^{2}+\left(\frac{m_{i}^{2}}{\theta_{i} n \theta_{i}^{2} p^{2}}\right)^{2}\left(\sigma_{p} / p\right)^{2} .
$$

It is reasonable to assume that the uncertainty of the Cherenkov angle is this width divided by the square root of the number of rings:

$$
\sigma_{\theta_{i}}^{2}=w_{i}^{2} / N_{i}
$$

The sum in quadrature of the angular uncertainties is then 


$$
N_{t o t} \sigma_{\theta}^{2}=w_{p}^{2} / \eta_{p}+w_{K}^{2} / \eta_{K}+w_{\pi}^{2} / \eta_{\pi}
$$

where $N_{\text {tot }}=N_{p}+N_{K}+N_{\pi}$ and $\eta_{i}=N_{i} / N_{\text {tot }}$. Given that $\eta_{p}+\eta_{K}+\eta_{\pi}=1$, it is trivial to show that the above is minimized when

$$
\eta_{i}=\sigma_{\theta_{i}} /\left(\sigma_{\theta_{p}}+\sigma_{\theta_{K}}+\sigma_{\theta_{\pi}}\right) .
$$

The optimal trigger fractions as well as the expected statistical uncertainty as a function of momentum can then be calculated using Eqns. 6.15, 6.16, 6.18, and 6.19. They are shown in Figures 6.1 and 6.2 assuming $\sigma_{n}=7 \times 10^{-6}$ and $\sigma_{p} / p=0.01$. The optimal fractions are approximately $35 \%$ pions, $45 \%$ kaons, and $20 \%$ protons.

These calculations suggest that the optimal momentum for this measurement is about $40 \mathrm{GeV} / c$. The expected statistical uncertainty is not significantly improved at lower momenta. Also, the protons would be too close to threshold, as evidenced by existing $35 \mathrm{GeV} / c$ data in which proton ring are very wide and have few PMT hits. Finally, above $50 \mathrm{GeV} / c$ pion and kaon rings begin to overlap, so it would be advantageous to keep momentum low enough that this does not happen.

The final goal is to achieve a precision of less than $100 \mathrm{ppm}$ so as to help resolve ambiguity in the current set of charged kaon mass measurements. With 10 million events at $40 \mathrm{GeV} / c$, we expect a statistical uncertainty of approximately $40 \mathrm{ppm}$. MIPP was well positioned to collect a data set of this 


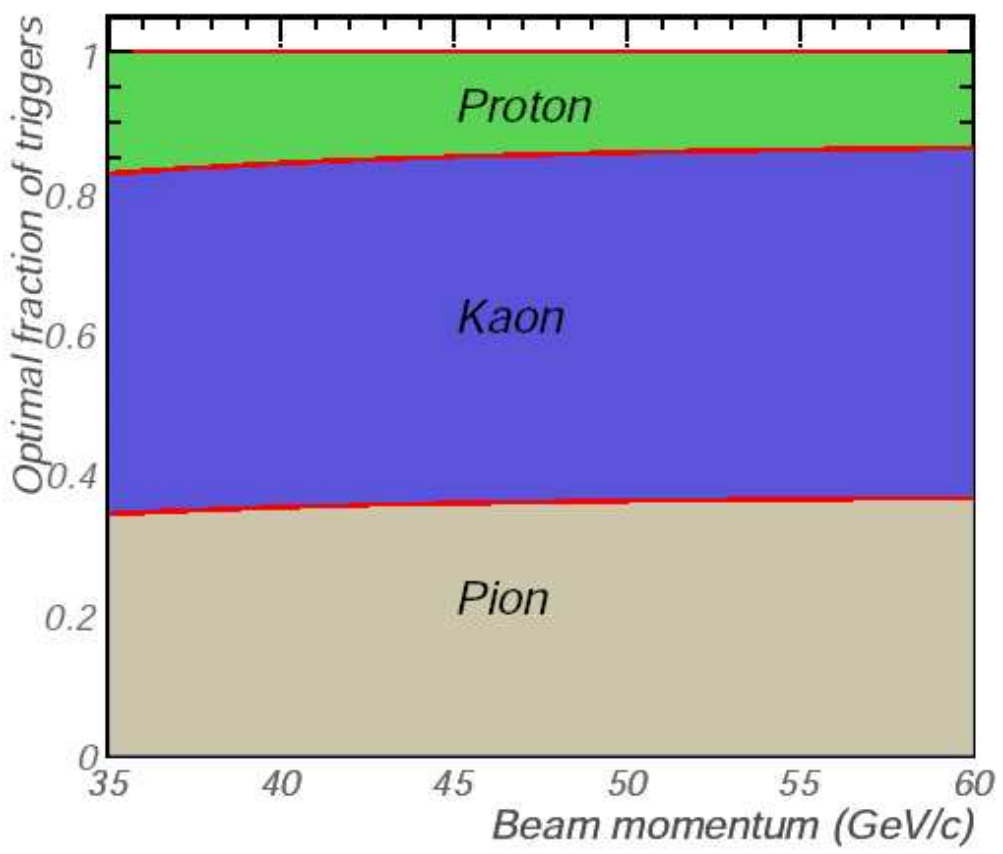

Figure 6.1: Optimal fractions for kaon mass measurement. 


\section{Expected statistical error}

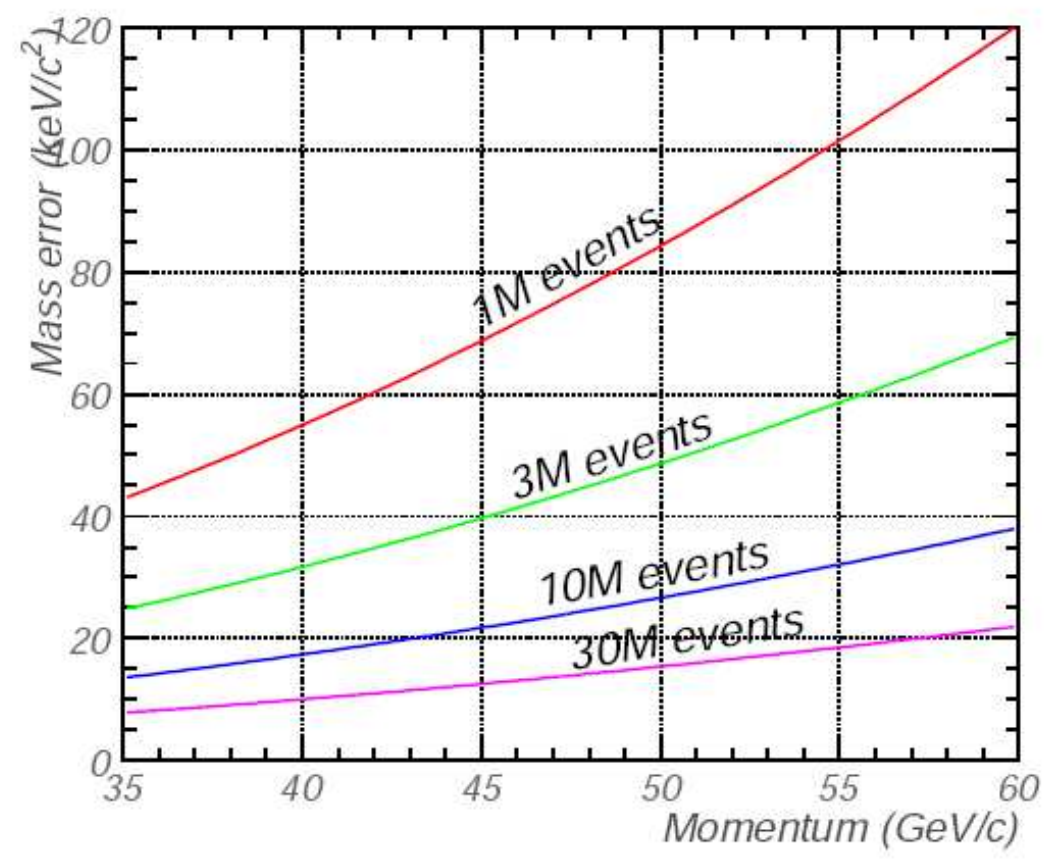

Figure 6.2: Expected statistical uncertainty for kaon mass measurement. 
size, meaning that our goal could be achieved with sufficient understanding of systematic uncertainties.

The concept of a ring where all the Cherenkov light is emitted at a single angle is an oversimplification. In reality light is distributed over a range of Cherenkov angles due to dispersion of the radiator. A more careful treatment of RICH rings which takes this into account is needed for a proper analysis. This is discussed in Chapter 10. 


\section{Chapter 7}

\section{Data Reconstruction}

\section{$7.1 \quad$ Tracking}

MIPP uses two methods to fit the vast majority of tracks: straight lines and track templates. The latter method is needed when tracks pass through a magnetic field region. As such, it is not used in this analysis. For a more detailed discussion of track fitting, including template tracks, see [27].

The only tracking elements active when this data set was taken were the wire chambers, which are divided into three sets of three each: BC123, DC123, and DC4/PWC56. Since curvature due to magnetic field is negligible in the regions between the magnets, before the Jolly Green Giant and after Rosie, straight line segments can be fit in each of the three chamber sets.

Different views are defined for different locations along the beam axis (三z axis). These views represent different orientations in the $x y$ plane. The 


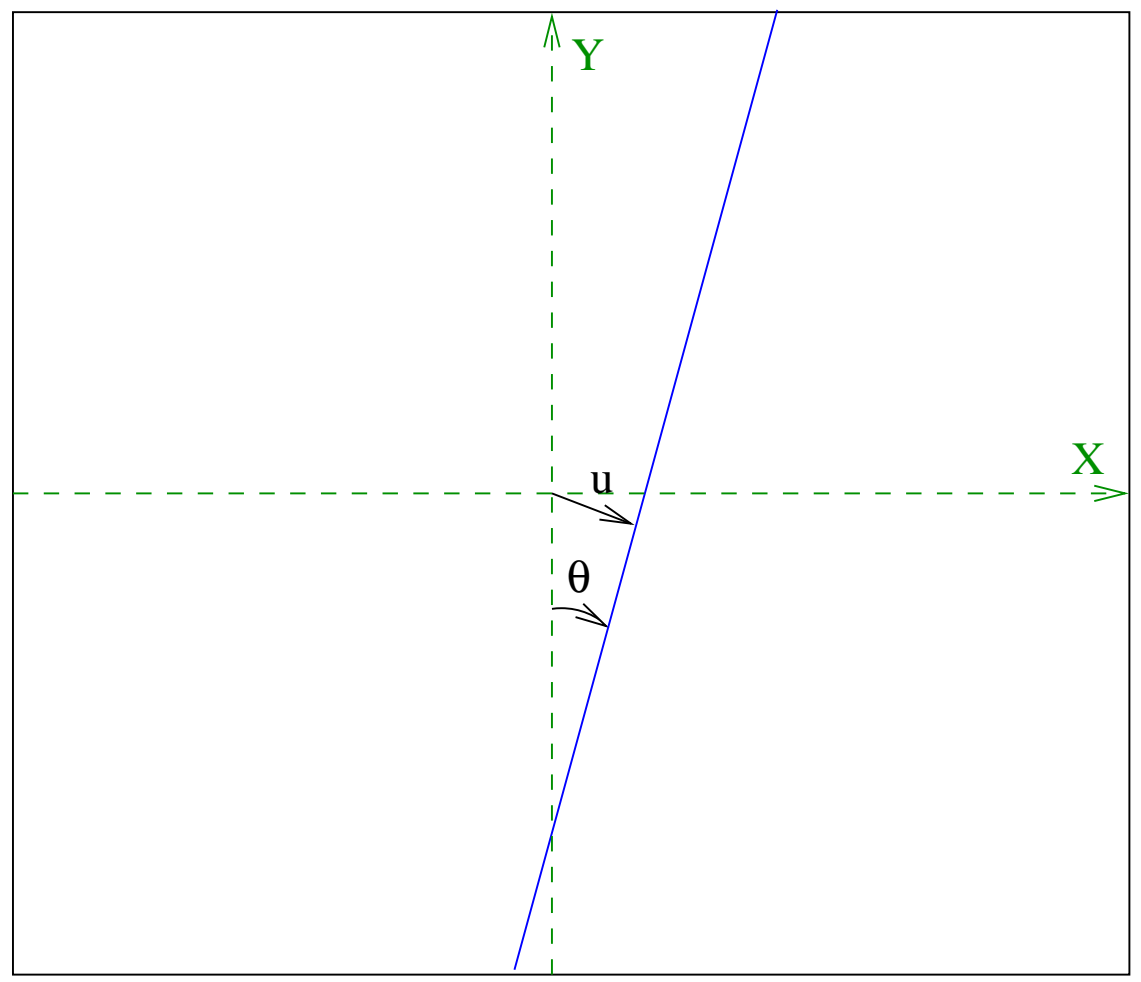

Figure 7.1: Wire plane geometry diagram.

convention is to define the angle of a wire, $\theta$, relative to the y-axis. Thus vertical wires, which measure position along $x$, have $\theta=0$ with $\theta$ increasing clockwise. Horizontal wires, which measure position along $y$, have $\theta=-90^{\circ}$. An $x, y$ position measurement can be transformed into a given wire view, as shown in Figure 7.1 via

$$
u=x \cos \theta-y \sin \theta
$$




\subsubsection{Straight Line Fits}

Defining a straight line requires 5 parameters: one 3-dimensional point and two slopes. For MIPP, this done using two points, $\left(x_{1}, y_{1}, z_{1}\right)$ and $\left(x_{2}, y_{2}, z_{2}\right)$, corresponding to the minimum and maximum $z$ positions of the points to be used in the fit. The task is then to fit for the four remaining parameters, $\vec{p}=\left(x_{1}, y_{1}, x_{2}, y_{2}\right)$.

As a function of the $z$ position, a line is described by

$$
\begin{aligned}
& x(z)=\frac{x_{1}\left(z-z_{2}\right)-x_{2}\left(z-z_{1}\right)}{z_{1}-z_{2}} \\
& y(z)=\frac{y_{1}\left(z-z_{2}\right)-y_{2}\left(z-z_{1}\right)}{z_{1}-z_{2}}
\end{aligned}
$$

Using Eqn. 7.1 we can than transform to a given view through

$$
u(z ; i)=\sum_{j=1}^{4} c_{i j} p_{j},
$$

where

$$
\vec{c}_{i}=\left(\begin{array}{c}
\frac{z-z_{2}}{z_{1}-z_{2}} \cos \theta_{i} \\
-\frac{z-z_{2}}{z_{1}-z_{2}} \sin \theta_{i} \\
-\frac{z-z_{1}}{z_{1}-z_{2}} \cos \theta_{i} \\
\frac{z-z_{1}}{z_{1}-z_{2}} \sin \theta_{i}
\end{array}\right)
$$

A least-squares method is then used to determine the track parameters. 
With $N$ measurements we have

$$
\chi^{2}=\sum_{i=1}^{N} w_{i}\left(u_{i}-\sum_{j} c_{i j} p_{j}\right)^{2}
$$

where $w_{i}$ is the weight assigned to wire $i$. The solution, then, will minimize $\chi^{2}$. Mathematically,

$$
\frac{\partial}{\partial p_{k}} \chi^{2}=2 \sum_{N} w_{i} c_{i k}\left(\sum_{j} c_{i j} p j-u_{i}\right)=0,
$$

which can be written in matrix form as

$$
\vec{v}=\mathbf{M} \tilde{\mathbf{p}},
$$

where

$$
\begin{gathered}
v_{j}=\sum_{i} w_{i} u_{i} c_{i j}, \\
M_{j k}=\sum_{i} w_{i} c_{i j} c_{i k} .
\end{gathered}
$$

Solving gives

$$
\begin{gathered}
\vec{p}=\mathbf{M}^{-1} \tilde{\mathbf{v}} \\
\chi^{2}=\sum_{i} u_{i} w_{i}^{2}-\vec{p} \cdot \vec{v} .
\end{gathered}
$$

$M$ is the inverse of the covariance matrix,

$$
\frac{1}{2} \frac{\partial^{2}}{\partial p_{i} \partial p_{j}} \chi^{2}=M_{i j},
$$


so the parameter uncertainties are given by

$$
\sigma_{p_{i}}^{2}=M_{i j}^{-1} .
$$

\subsection{Wire Chambers}

As previously stated, wire chambers are grouped into three sets of three each. For the kaon mass analysis, chamber reconstruction first attempts to form straight line track segments using the most downstream set, DC4/PWC56. If this fails, the next most downstream chamber, in this case DC3, is included in the fit. Each time the fit fails, the next most downstream chamber is included until a track segment is formed.

Another unique feature of track reconstruction for this analysis is that only wires near the beam axis were considered, since the data consists only of straight beam tracks. This resulted in a track segment being reconstructed for each event. Otherwise, a track segment was successfully reconstructed in approximately only two-thirds of all events, before other cuts.

\subsubsection{Wire Clusters}

A cluster is a group of one or more contiguous wires which all have similar hit times. For drift chamber cluster size is limited to two wires, whereas there is no size limit in PWCs. Ringing in the chamber electronics would occasionally cause all 8 wires from a given preamplifier to be hit. These wires 
were excluded from the track fitting.

For each wire cluster, the geometric center is found in the local chamber reference frame by

$$
u_{\text {loc }}=D_{\text {plane }}\left(W-W_{0}\right),
$$

where $D_{\text {plane }}$ is the wire spacing and $W_{0}$ is the hypothetical wire passing through $u=0$ in the local frame. A given point on the wire $\left(u \cos \theta_{l o c}\right.$, $\left.-u \sin \theta_{l o c}, z_{l o c}\right)$ can be transformed to the global coordinate system $\left(x_{g l o b}\right.$, $\left.y_{g l o b}, z_{g l o b}\right)$. Global coordinates are then transformed into the appropriate wire view using Eqn. 7.1, giving the global $u$ of the cluster.

\subsubsection{Wire Crosses}

Using Eqn. 7.1, the intersection of two wires is

$$
\begin{aligned}
& x=\frac{u_{1} \sin \theta_{2}-u_{2} \sin \theta_{1}}{\cos \theta_{1} \sin \theta_{2}-\sin \theta_{1} \cos \theta_{2}} \\
& y=\frac{u_{1} \cos \theta_{2}-u_{2} \cos \theta_{1}}{\cos \theta_{1} \sin \theta_{2}-\sin \theta_{1} \cos \theta_{2}}
\end{aligned}
$$

When a cross has more than two wires, its position is found using the least squares approach described by Eqn. 7.5 with

$$
\vec{p}=\left(\begin{array}{c}
x \\
y
\end{array}\right), \vec{c}=\left(\begin{array}{c}
\cos \theta_{i} \\
-\sin \theta_{i}
\end{array}\right) .
$$


The following algorithm was used to find wire crosses:

1. Find intersection of two clusters that don't belong to the same wire cross and are from different planes. Confirm that this point is within the active chamber volume.

2. Search for clusters consistent with this point in the remaining views of the chamber

3. Fit for wire cross position using all available views. If $\chi^{2}$ is not acceptable, choose the combination of 3 planes with the best $\chi^{2}$.

Any degeneracies are then discarded. More specifically, crosses which are a subset of another cross, as well as crosses in which all wires all also in crosses which have more clusters.

\subsubsection{Track Segments}

The general approach to finding track segments was to evaluate all combinations of chamber points and keep the ones which had an acceptable $\chi^{2}$ and information from all three of the chambers being considered.

For this purpose, a data structure as defined containing the following information:

- Wire crosses contained in the segment

- Wire clusters contained in the segment 
- Minimum and maximum wire time

- Segment fit parameters

Each cross and cluster were also represented by data structures with the minimum and maximum wire time and a list of segments in which the cross or cluster was contained. This organization structure made it relatively easy and quick to determine: if two crosses are compatible; if the cross or cluster is compatible with a track segment; if two crosses are already part of a segment; or the number of clusters shared by different segments.

The algorithm for track segment finding was:

1. Consider each pair of crosses that are near the beam axis for each pair of chambers.

2. If the crosses have compatible times, proceed to step 3.

3. Fit a straight line to the clusters of the crosses and predict position at the third chamber.

4. Using this prediction, find the closest cross or cluster which is time compatible with the initial crosses and re-fit. If $\chi^{2}$ is acceptable, proceed to step 6 .

5. Find the closest time compatible wire cluster in each view of the third chamber, re-fitting for each. If there is one and only one acceptable cluster, proceed to step 6 . 
6. Save the track segment only if it has the minimum required number of clusters.

The next step in MIPP track reconstruction was to match track segments for the different chamber triplets to form track candidates which would then be combined with track fits from the TPC. The TPC was not active during data taking for this analysis and only a single track segment using the downstream chambers was reconstructed, so the remainder of the track reconstruction is not relevant.

\section{3 $\quad$ Ring Fitting}

RICH ring reconstruction in MIPP makes use of two different approaches to fitting circles to PMT hits. The first applies a Hough transform to the set of PMT hits in an event, and is thus independent of tracking. The second uses track reconstruction to provide starting values for ring center and radius. An algorithm based on deterministic annealing is then applied. The former method is used in this analysis for alignment of RICH mirrors which is discussed in Chapter 9. The latter is unused in this analysis and will not be discussed further.

Circle fitting is done in two passes. The first loops over all sets of three hits. Each set defines a circle, and the $x$ and $y$ position of the center and the radius are histogrammed. Peaks are searched for and if any are found, they are saved as potential ring candidates. 
The second pass then takes each ring candidate and performs a $\chi^{2}$ fit on all the points with the ring candidate parameters as seeds. $\chi^{2}$ in this case is actually just a sum of residuals squared. This procedure is then iterated while at each step paring the number of PMT hits by weighting down those that contribute most to the $\chi^{2}$. The final improved ring fit is then saved. 


\section{Chapter 8}

\section{Data}

\subsection{Running Conditions}

Running conditions for the charged kaon mass measurement differed from normal MIPP running. Many detector systems were not needed for the measurement and were not active. It was also necessary to remove systems to increase the data rate. The TPC is the slowest detector to read out and would have limited the data rate to about $30 \mathrm{~Hz}$. The EMCAL also would have negatively impacted the data rate. By removing these systems, the data rate was increased by a factor of approximately 20. The detector systems not used in the experiment were: TPC, CKOV, TOF, and EMCAL. Those active were drift and wire chambers, RICH, and HCAL. Prescales were set so that relative fractions would be as close as possible to the optimum values justified in Chapter 6. 


\begin{tabular}{l|cccc}
\hline \hline Data Set & Protons & Kaons & Pions & Total \\
\hline $37 \mathrm{GeV}$ & 637451 & 769276 & 810765 & 2217492 \\
$40 \mathrm{GeV}$ & 1104688 & 1035826 & 1196312 & 3336826 \\
$42 \mathrm{GeV}$ & 413506 & 335265 & 338196 & 1086967 \\
$56 \mathrm{GeV}$ & 178353 & 124219 & 160514 & 463086 \\
$60 \mathrm{GeV}$ Field Off & 1190451 & 524624 & 949961 & 2665036 \\
$60 \mathrm{GeV}$ Field On & 900605 & 384562 & 688882 & 1974049 \\
$63 \mathrm{GeV}$ & 132212 & 48135 & 75010 & 255357 \\
\hline Totals & 4557266 & 3221907 & 4219640 & 11998813 \\
\hline \hline
\end{tabular}

Table 8.1: Summary of event totals for each data set.

Although the optimal momentum was determined to be $40 \mathrm{GeV} / c$, data was taken at several different momenta. The reason for this is that most of the data was taken opportunistically, usually when the analysis magnets were down. As such, most the data was taken with no magnetic field. Data was taken at the following momenta: $37 \mathrm{GeV} / c, 40 \mathrm{GeV} / c, 42 \mathrm{GeV} / c, 56$ $\mathrm{GeV} / c, 60 \mathrm{GeV} / c$, and $63 \mathrm{GeV} / c$. Some of the $60 \mathrm{GeV} / c$ was taken with analysis magnets on.

Table 8.1 summarizes the amount of data taken. A total of just under 12 million events with clear PID from the beam Cherenkov counters were collected, exceeding the goal of 10 million. There were an additional 2.3 million events which did not have a clear PID. 


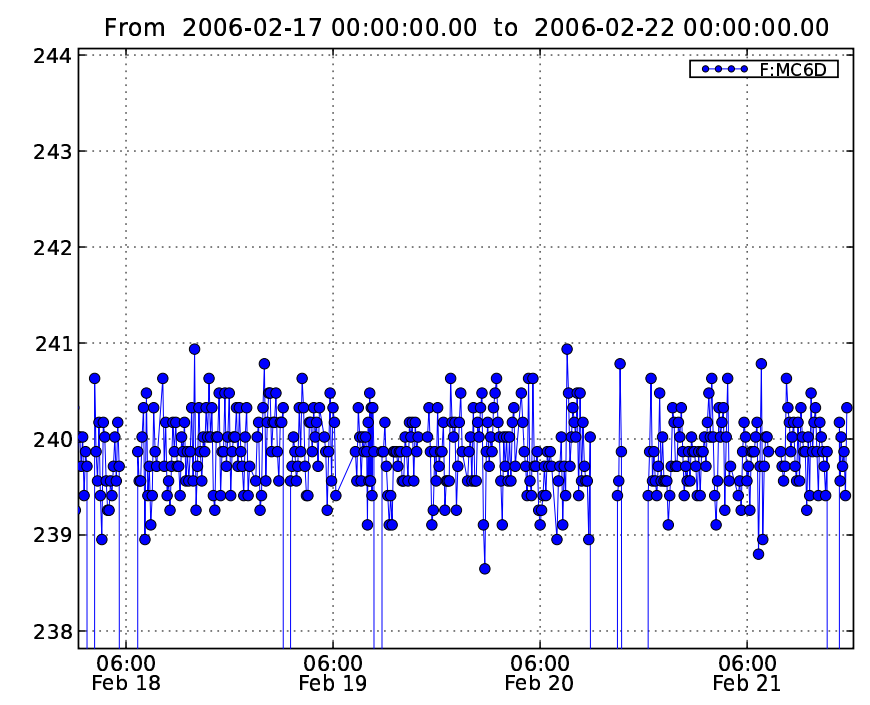

Figure 8.1: Stability of momentum selecting magnet current in $\mathrm{mA}$. The points where the current suddenly drops correspond to missed spills.

\subsection{Data Quality}

\subsubsection{Magnet Current}

Figure 8.1 shows the current for the momentum selecting magnet in the primary beamline during $40 \mathrm{GeV} / c$ data taking. During this time period, the magnet current was ramped up on each spill, the value recorded, and then ramped down again. This was done because of a short occurring in one of the dipoles when heated. The regions of the plot where the current suddenly drops correspond to times when MIPP did not receive spills. The current varied by less than $1 \%$ during data taking. 


\subsection{2 $\mathrm{CO}_{2}$ Density}

Pressure and temperature of $\mathrm{CO}_{2}$ inside the $\mathrm{RICH}$ vessel were logged every 10 minutes during the run. There were two temperature sensors, one at the upstream end and one near the downstream end. The upstream sensor consistently reads out about 3 degrees higher since it is near the readout electronics. Temperature varied by about 2 degrees Celsius during low momentum running (Figure 8.3), with a corresponding pressure variation from about 14.8 psi to 15.1 psi (Figure 8.2). Temperature was more consistent during high momentum running, varying by less than 1 degree (Figure 8.6).

Density was calculated using the Beattie-Bridgeman equation of state:

$$
P=R T(1-\epsilon) \frac{V+B}{V^{3}}-\frac{A}{V^{2}}
$$

where $A=A_{0}\left(1-\frac{a}{V}\right), B=B_{0}\left(1-\frac{b}{V}\right), \epsilon=\frac{C}{V T^{3}}, V$ is the volume in mole ${ }^{-1}, P$ is pressure in atm, $T$ is absolute temperature, $R$ is the gas constant, and the other parameters are characteristic of the gas. Solving for density requires the solution to a quartic equation [1].

The Beattie-Bridgeman equation of state was suggested by [39] as more accurate than the ideal gas law. Comparisons between the two are shown in Figures 8.4 and 8.7. The vertical axis is the ratio of density inside the RICH to density at STP. There is a difference of about $0.15 \%$ between the two. For high momentum running, the jumps in density and pressure (Figure 8.5)occur when the RICH was filled up periodically. 


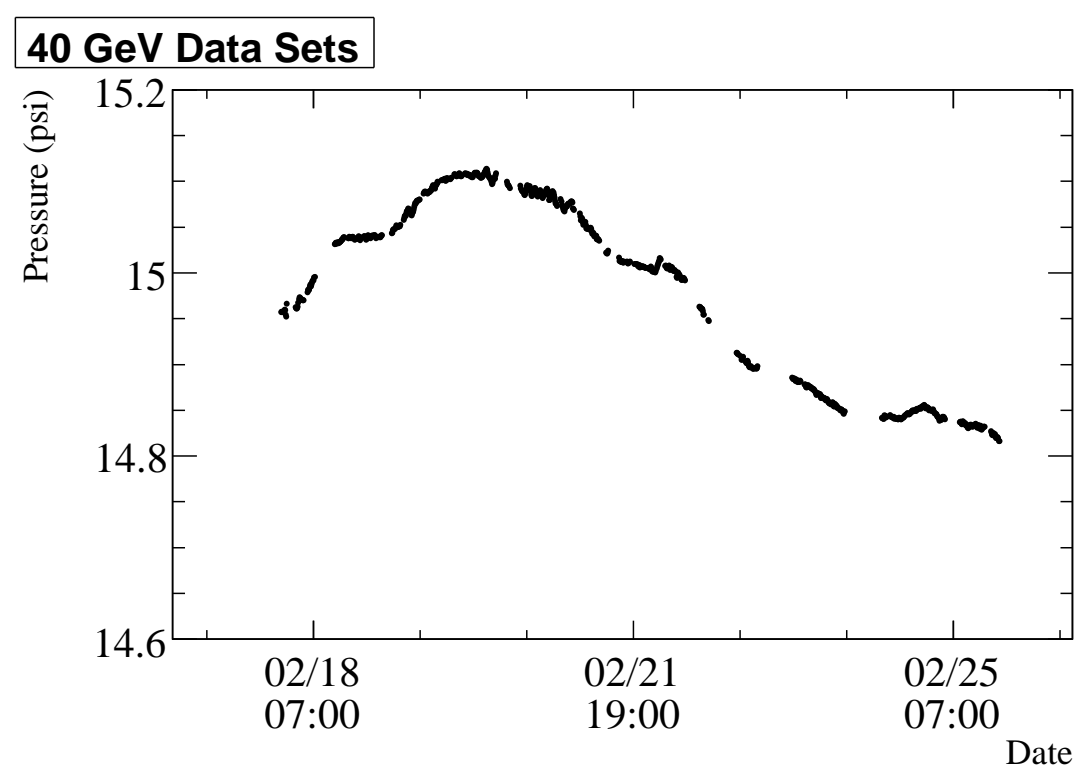

Figure 8.2: RICH vessel pressure during low momentum running.

\subsubsection{Finding Bad Channels}

Due to all of the handling of tubes during initial installation and again during testing and refurbishment after the fire, it was inevitable that some tubes would be lost. These bad channels were flagged on a run-by-run basis by measuring the occupancy. Occupancy, or occupation, is defined as the fraction of events in which a tube fired. Dead tubes were determined from in-spill events, (Figure 8.8) and hot tubes from calibration events using the pulser and LED (Figure 8.9). Tubes with an occupancy less than $10^{-4}$ during spill events were flagged as dead and those above $10^{-1}$ during calibration events were deemed hot.

The number of bad channels flagged using this algorithm is shown in 


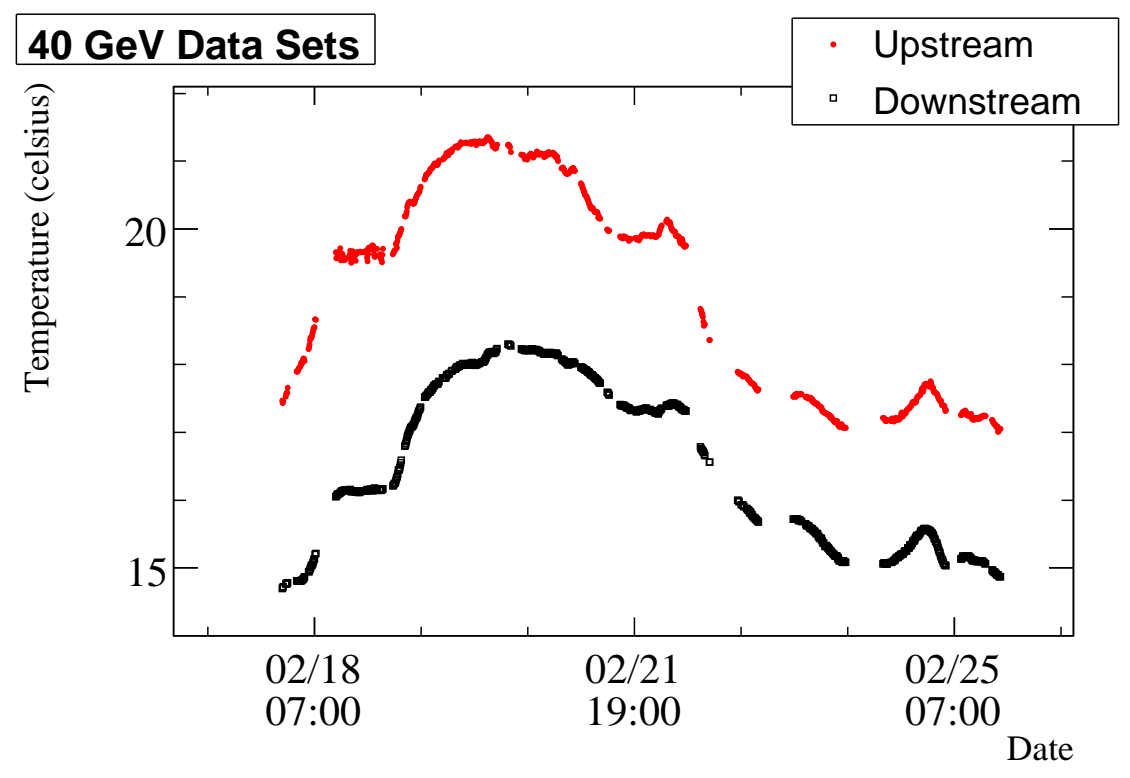

Figure 8.3: RICH vessel temperature during low momentum running. The top curve is the upstream probe and the bottom curve is the downstream probe. 


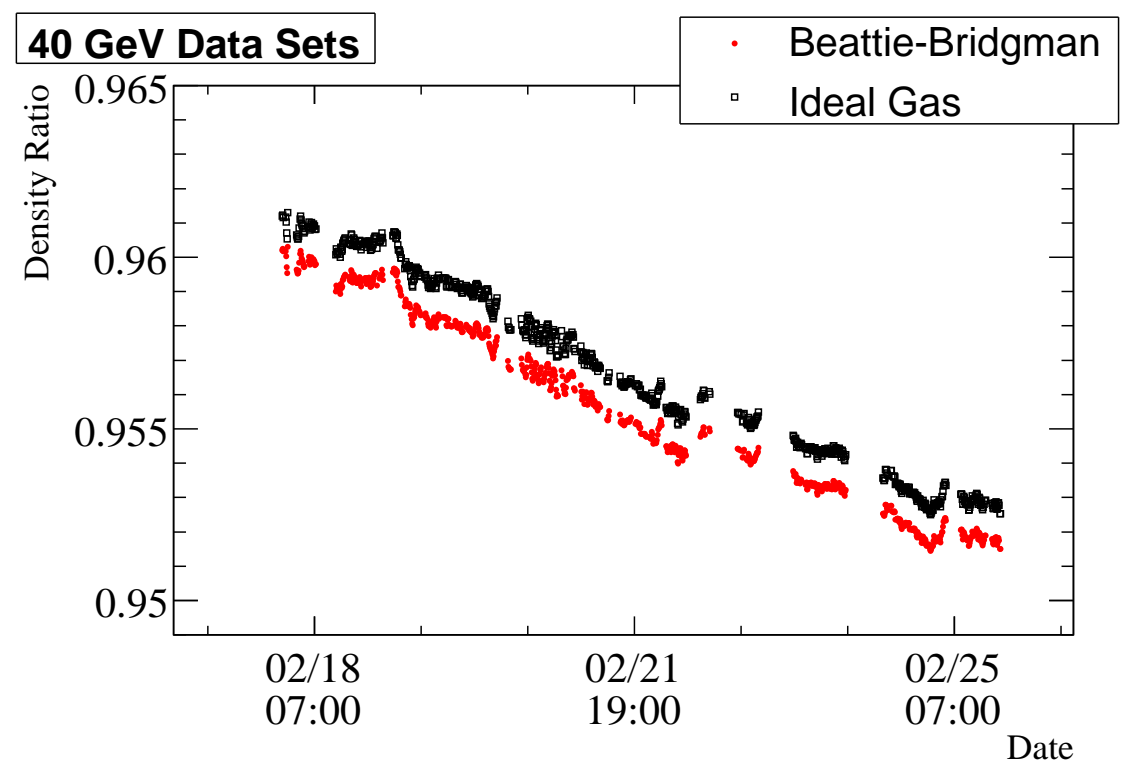

Figure 8.4: $\mathrm{RICH} \mathrm{CO}_{2}$ density relative to STP during low momentum running. The top curve is calculated assuming an ideal gas, while the bottom curve is from the Beattie-Bridgeman equation of state. 


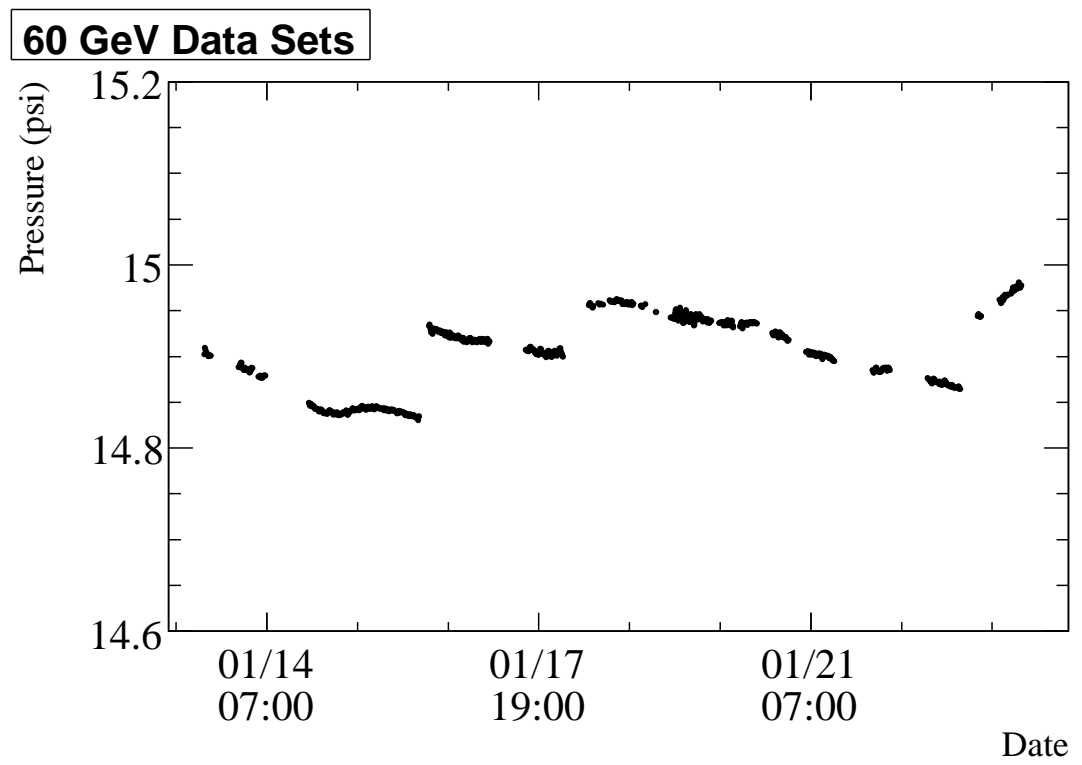

Figure 8.5: RICH vessel pressure during high momentum running.

Figure 8.10. The number of dead tubes is fairly stable and is around 100 . The reason many runs have more bad channels is due to events where many tubes in a column, often all 32, would fire. These hot columns come and go randomly from event to event. Attempts were made to reduce the frequency of hot column appearing in the data by slightly raising thresholds on RICH readout boards, though this did not entirely correct the problem. This problem was not seen outside of kaon mass running and was likely due to the higher rate. Runs during high momentum data taking (Figure 8.12) had a more frequent incidence of hot columns than low momentum runs (Figure 8.11).

Some columns would show up hot often enough to be caught by the bad 


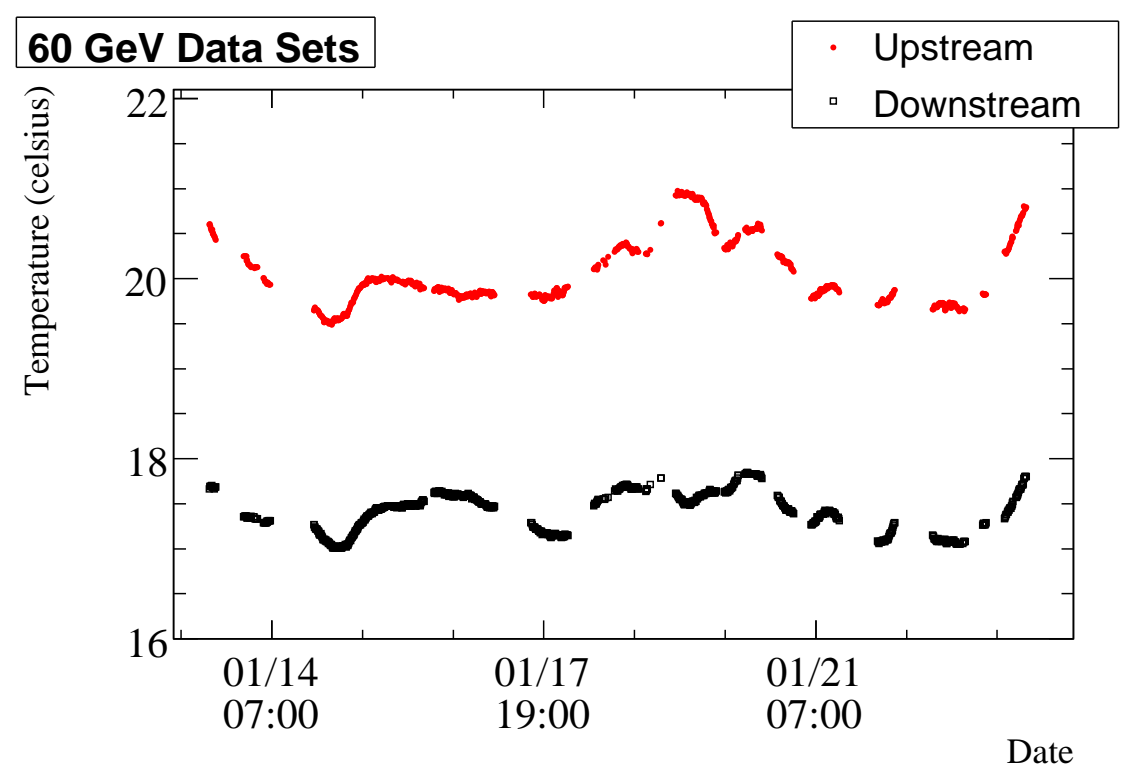

Figure 8.6: RICH vessel temperature during high momentum running. The top curve is the upstream probe and the bottom curve is the downstream probe. 


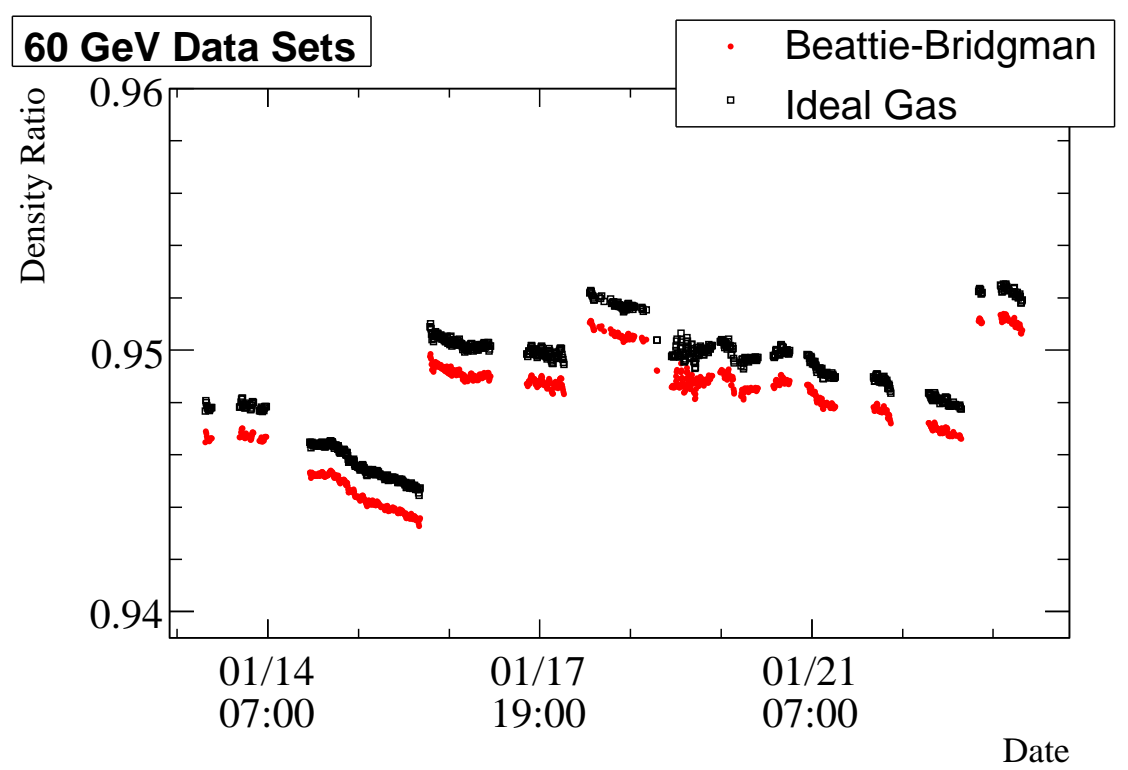

Figure 8.7: RICH $\mathrm{CO}_{2}$ density relative to STP during high momentum running. The top curve is calculated assuming an ideal gas, while the bottom curve is from the Beattie-Bridgeman equation of state. 


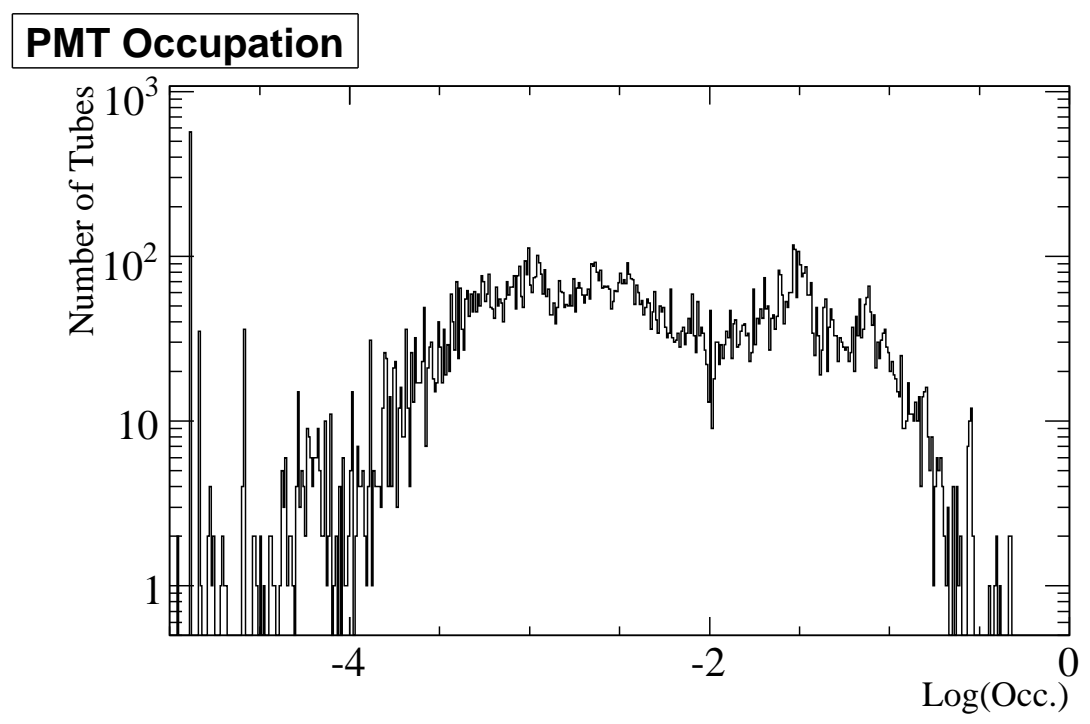

Figure 8.8: PMT occupation for in-spill events during kaon mass data taking.

\section{PMT Occupation}

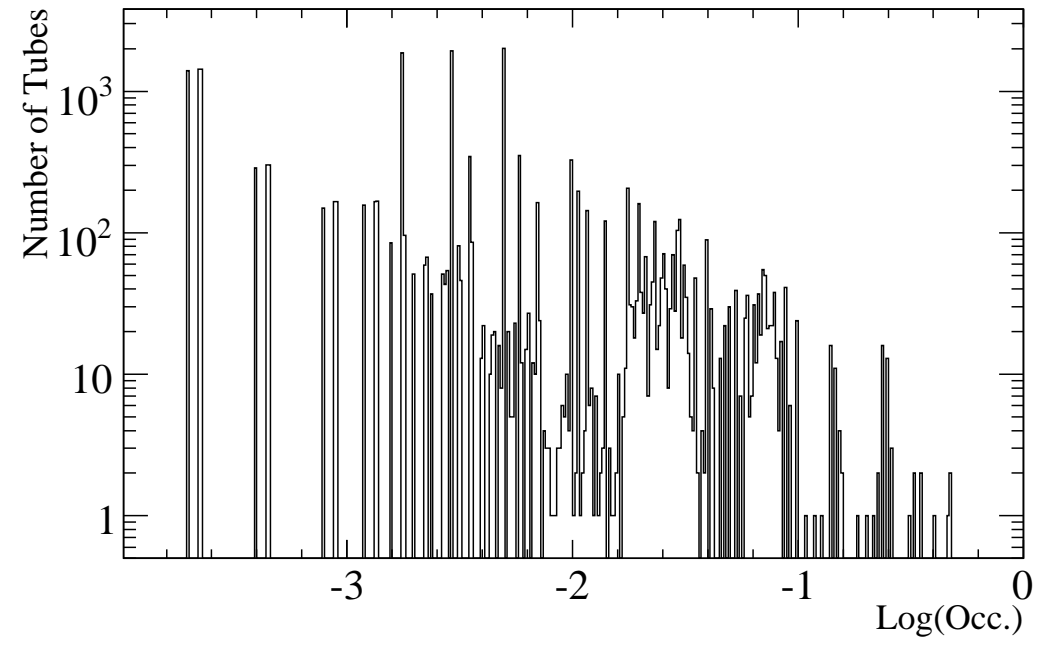

Figure 8.9: PMT occupation for calibration events during kaon mass data taking. 


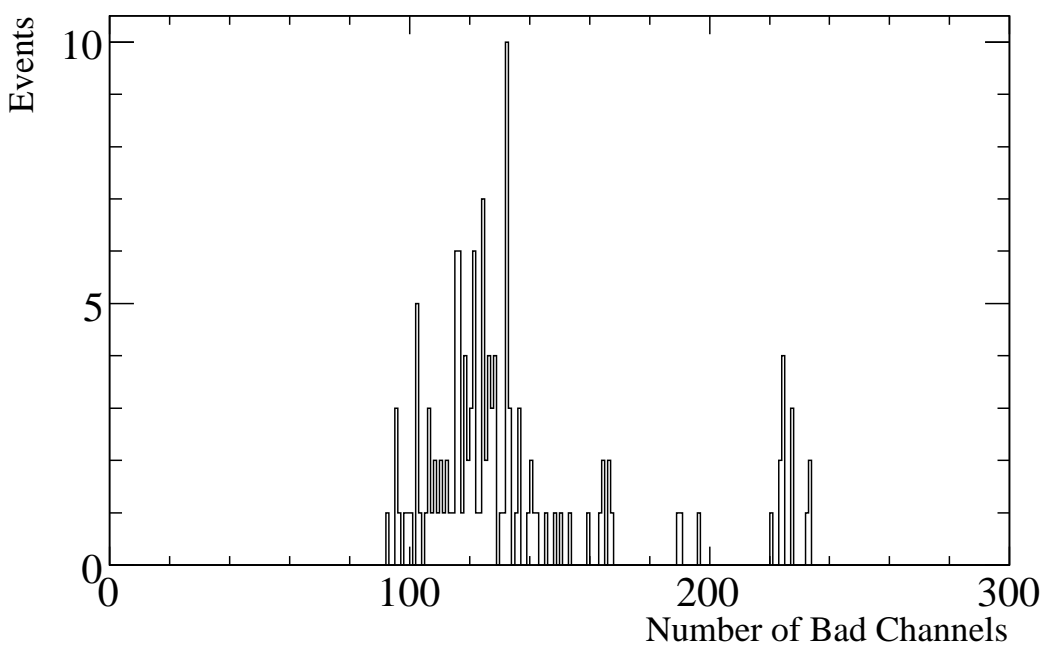

Figure 8.10: Number of bad channels per run during kaon mass data taking.

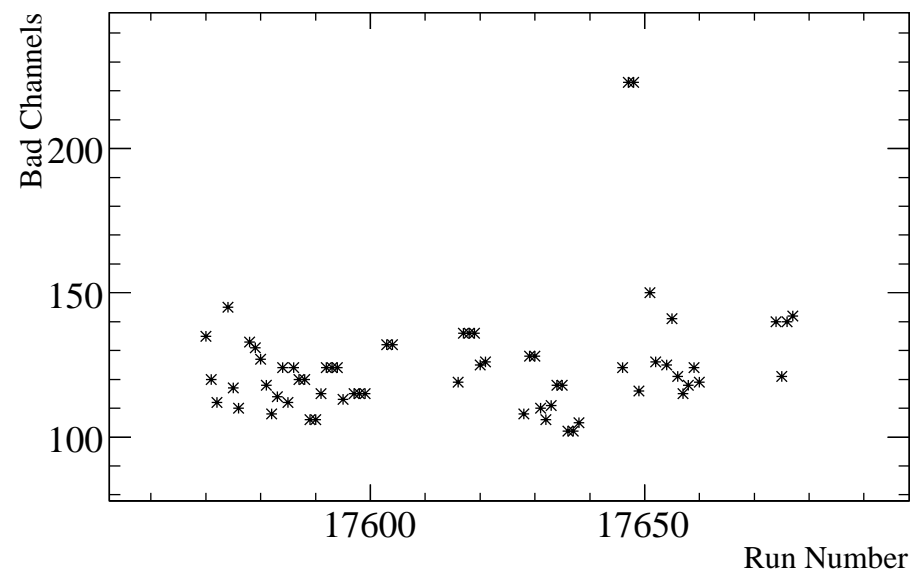

Figure 8.11: Number of bad channels vs. run during low momentum kaon mass data taking. The two runs above 200 had a high incidence of hot columns. 


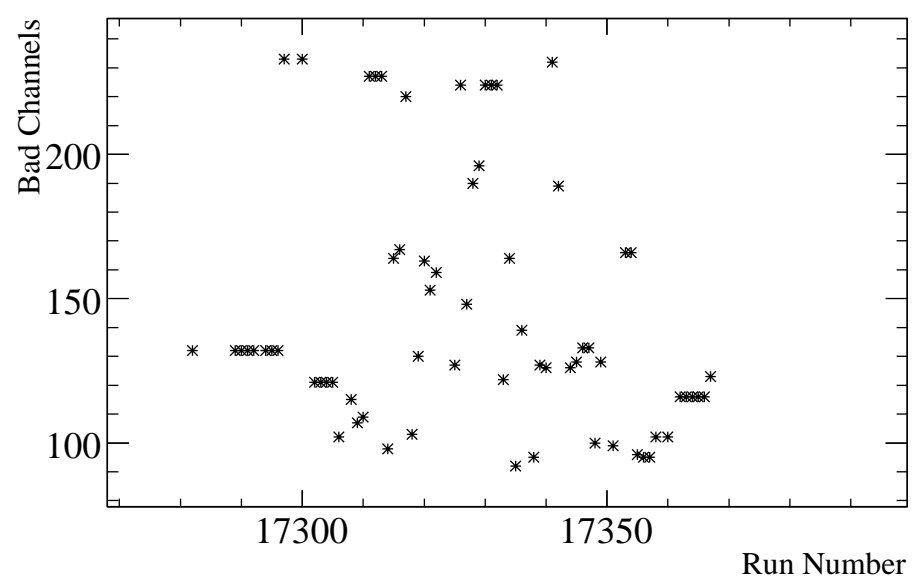

Figure 8.12: Number of bad channels vs. run during high momentum kaon mass data taking.

channel finder. The remaining were removed on an event by event basis by searching for columns where at least six tubes had fired. Sample event displays with hot columns are shown in Figure 8.13. Still, most events had no columns while a very small fraction had seven or more (see Figure 8.14). From Figure 8.15, most of the columns which were often hot were toward the center of the array. This proved especially problematic for proton events as hot columns would often appear near the edges of rings.

\subsubsection{PMT Noise Rate}

The average phototube noise rate was also determined from occupancy during calibration events. This was done using by combining pulser runs with 

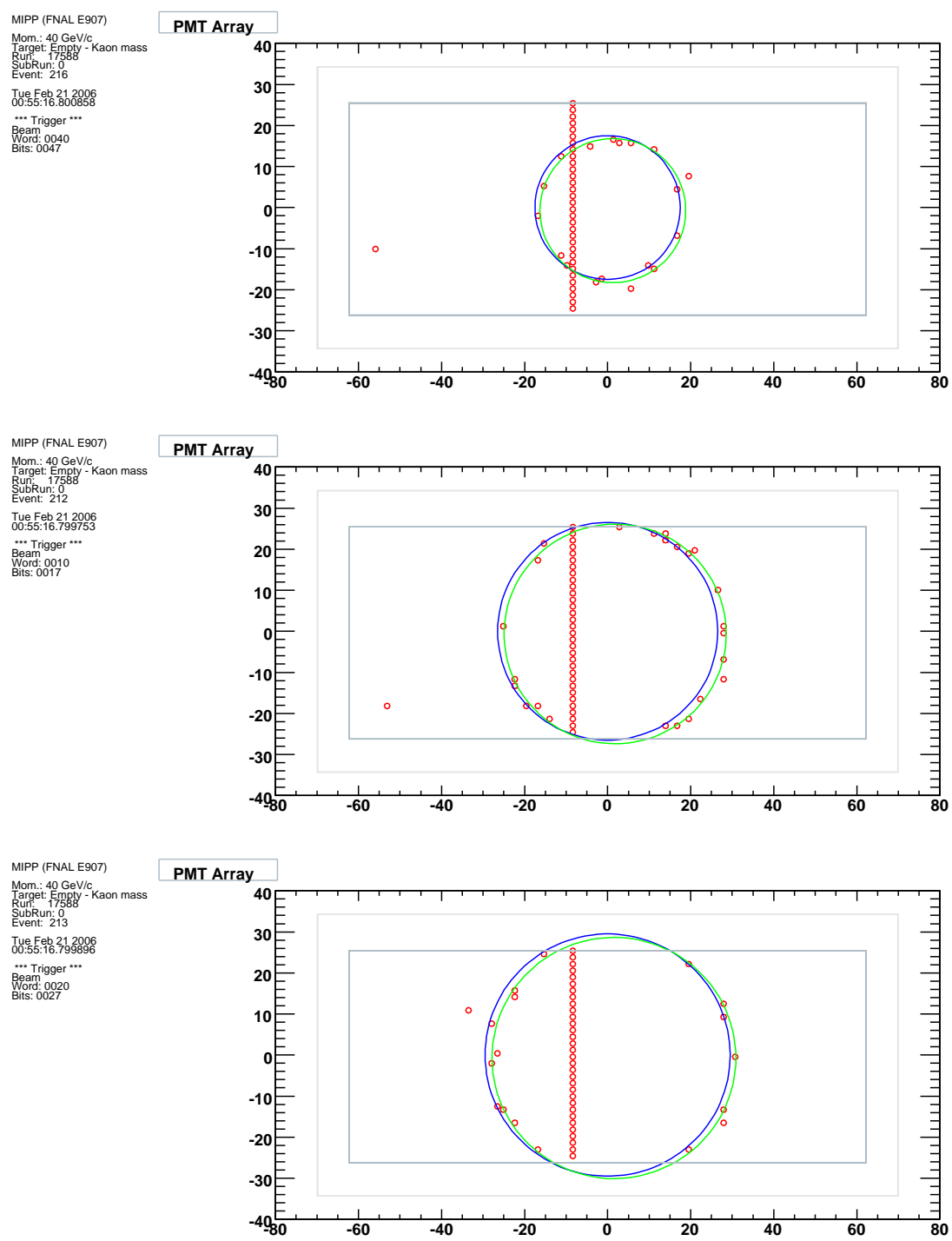

Figure 8.13: RICH event display showing hot columns. The small circles represent PMT hits. The larger circles are the initial and improved ring fits respectively. Top: proton event. Middle: kaon event. Bottom: pion event. 


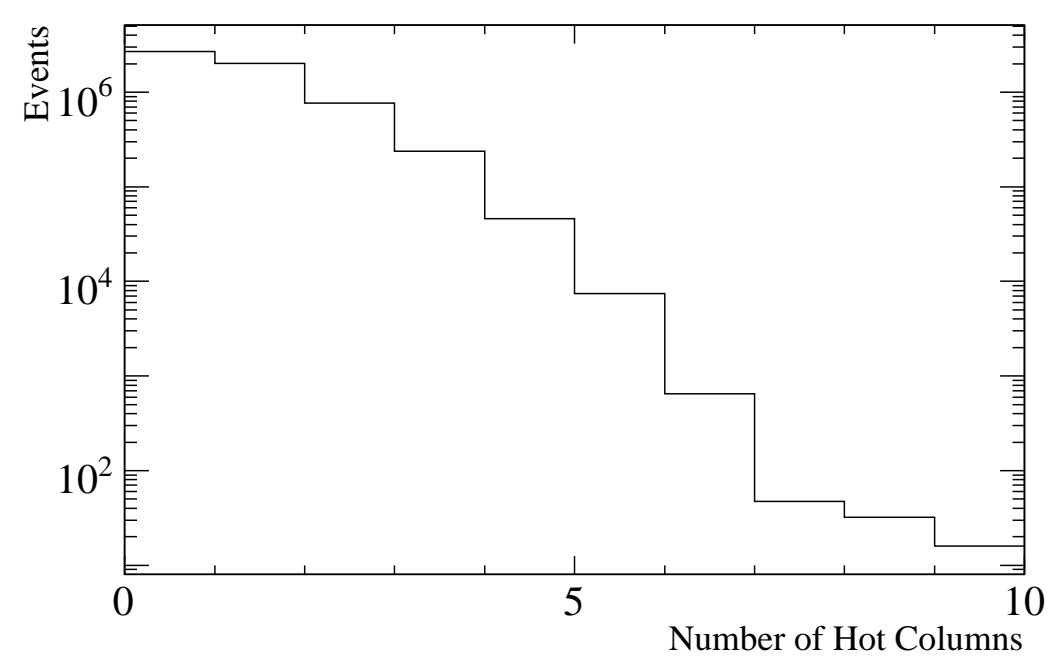

Figure 8.14: Number of hot columns per event during kaon mass data taking.

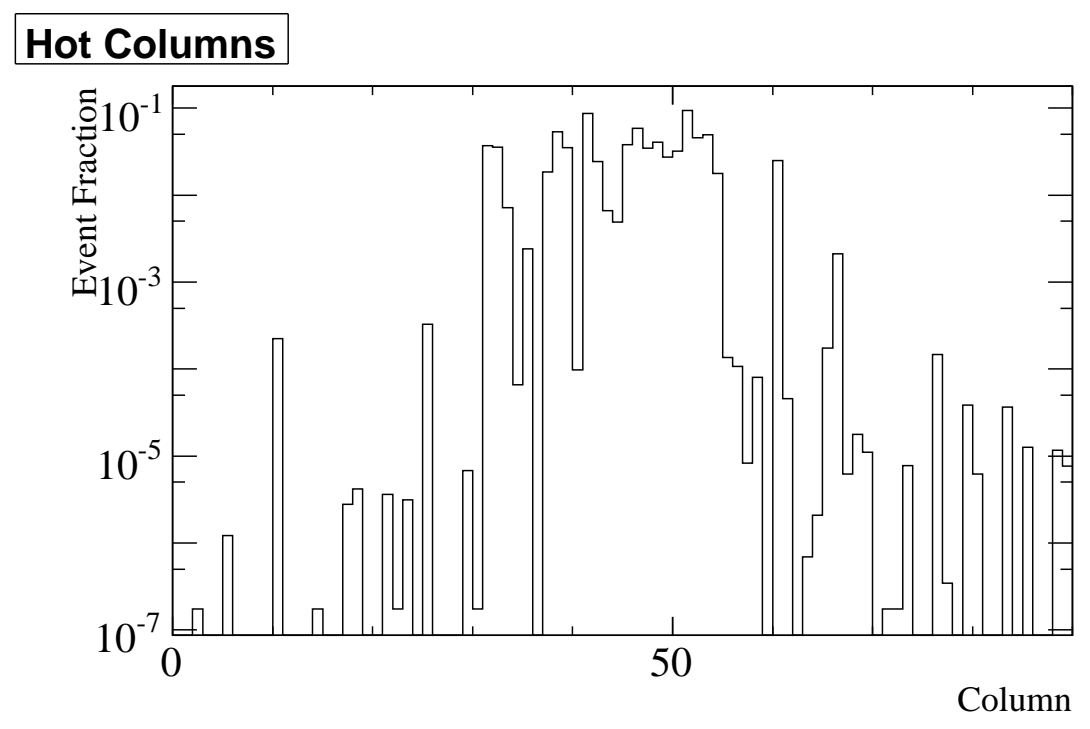

Figure 8.15: Fraction of events in which a column was hot. Most hot columns occur in the central region of the array. 


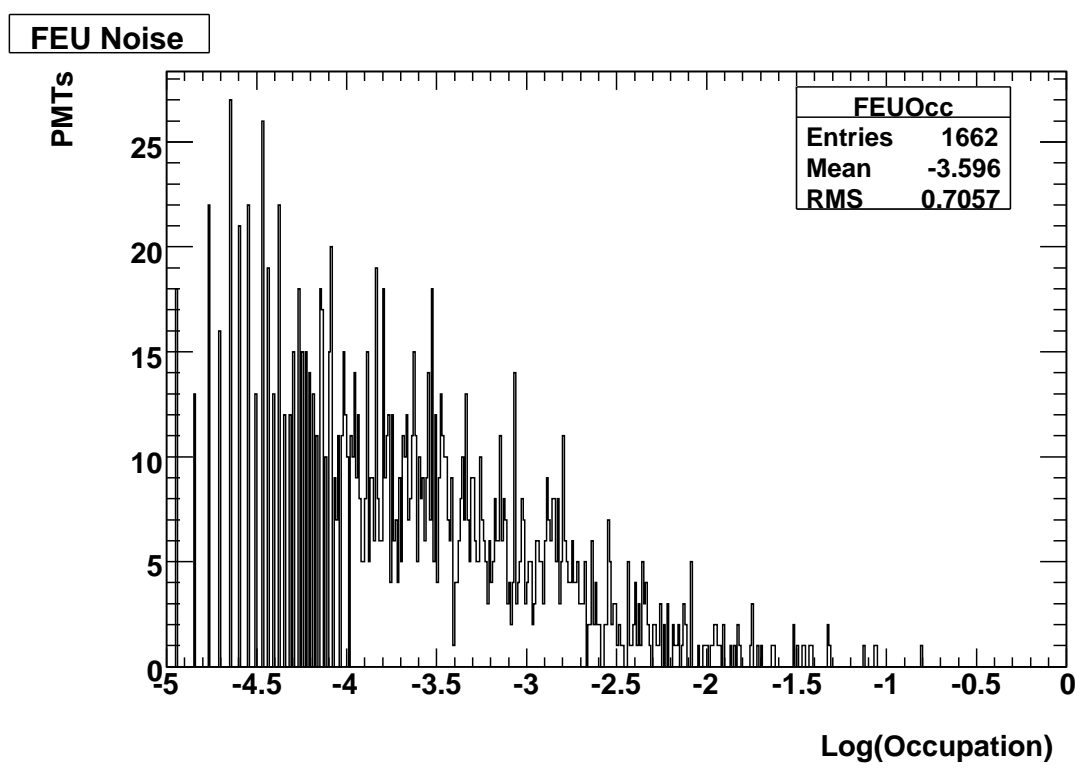

Figure 8.16: FEU tube occupancy for calibration events.

some of the longer production runs so that enough events were accumulated, approximately 350,000 in total. The RICH gate is $100 \mathrm{~ns}$ for a total readout time of 0.035 seconds. For FEU60 tubes, shown in Figure 8.16, the average noise rate was $2.5 \mathrm{kHz}$. For R760 tubes, shown in Figure 8.17, the average rate was $1.1 \mathrm{kHz}$.

\subsection{Data Selection}

Obtaining as pure a sample of good, clean beam events as possible is necessary for making an accurate, high precision measurement of the charged kaon mass. Accomplishing this is not as easy as simply relying on the beam 


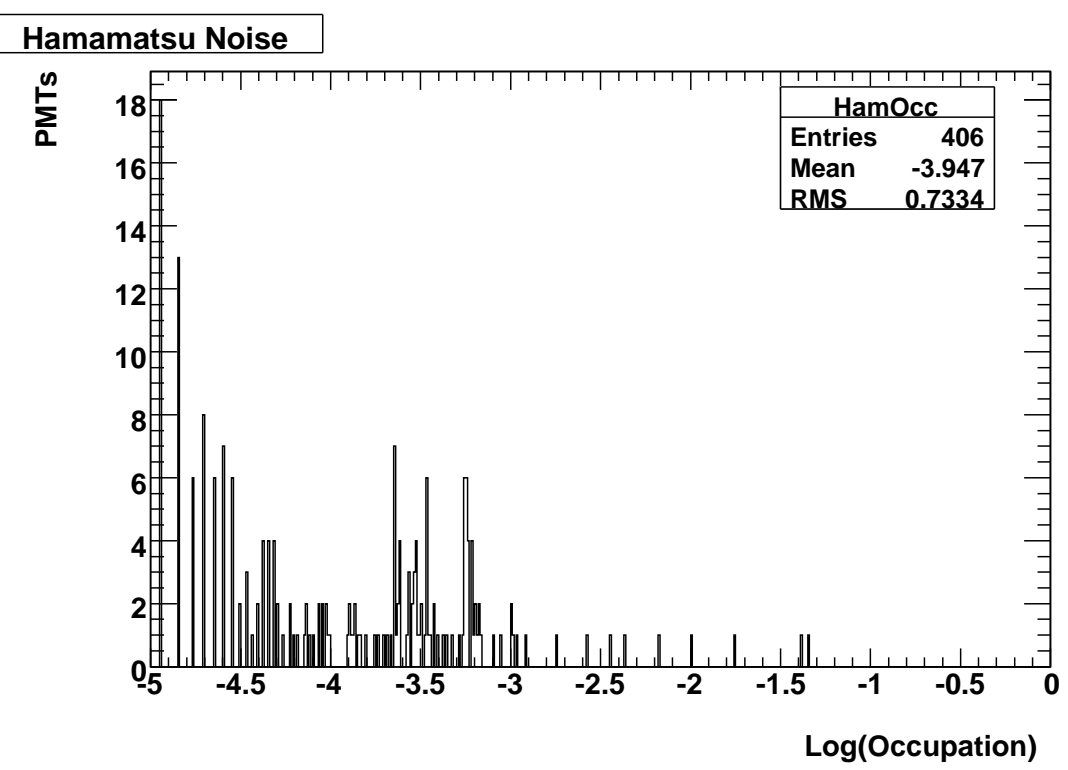

Figure 8.17: Hamamatsu tube occupancy for calibration events.

trigger, which is not $100 \%$ pure. Beam pileup, spray, and interactions occurring upstream of the RICH all need to be removed from the data set, as well as poorly fit track segments.

To filter out these unwanted events, cuts were made using information from the detectors available during this running period. For an event to be accepted, it must first show a clear signal from the beam Cherenkov counters indicating that a beam particle was detected and identified as either $\pi, K$, or $p$. Second, the interaction trigger must not have fired.

In addition, a cut on the HCAL ADC sum is made based on the overall distributions for each particle species seen in a given data set. This ensures that the beam event in question has a momentum within a reasonable range 


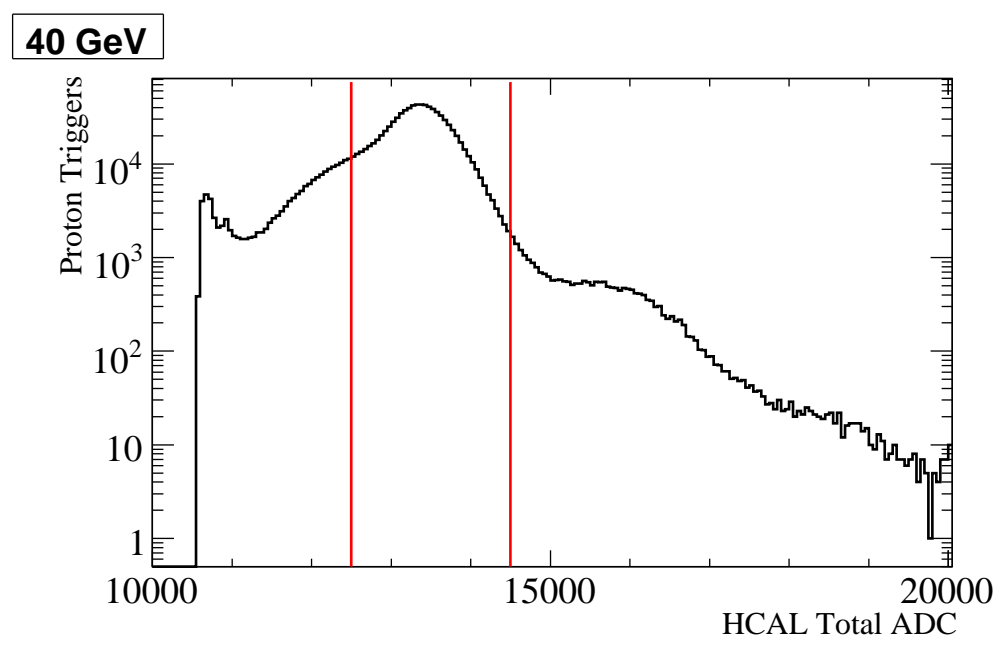

Figure 8.18: Hcal ADC sum for protons from the $40 \mathrm{GeV}$ data set. Events between 12500 and 14500 were kept, as indicated by the vertical lines.

and serves as an added check against interactions upstream of the RICH, as well as beam pileup. In each case, the distribution has a shoulder to the left of the main peak, likely due to decays or interactions. The shoulder to the right of the central peak is from beam pileup. The left-most peak is the sum of pedastals. Cuts were chosen to eliminate these shoulders, as indicated by the vertical lines. Distributions for $40 \mathrm{GeV} / c$ are shown in Figures 8.18-8.20. $60 \mathrm{GeV} / c$ distributions are shown in Figures 8.21-8.23. The HCAL and EMCAL were calibrated simultaneously. Since the EMCAL was not active, calibration constants were not available for these runs.

Cuts were also made on the reconstructed track segment. For the field off data set, the segment was required to use clusters in at least two of the three most downstream chambers. For field on, it was required that only the three 


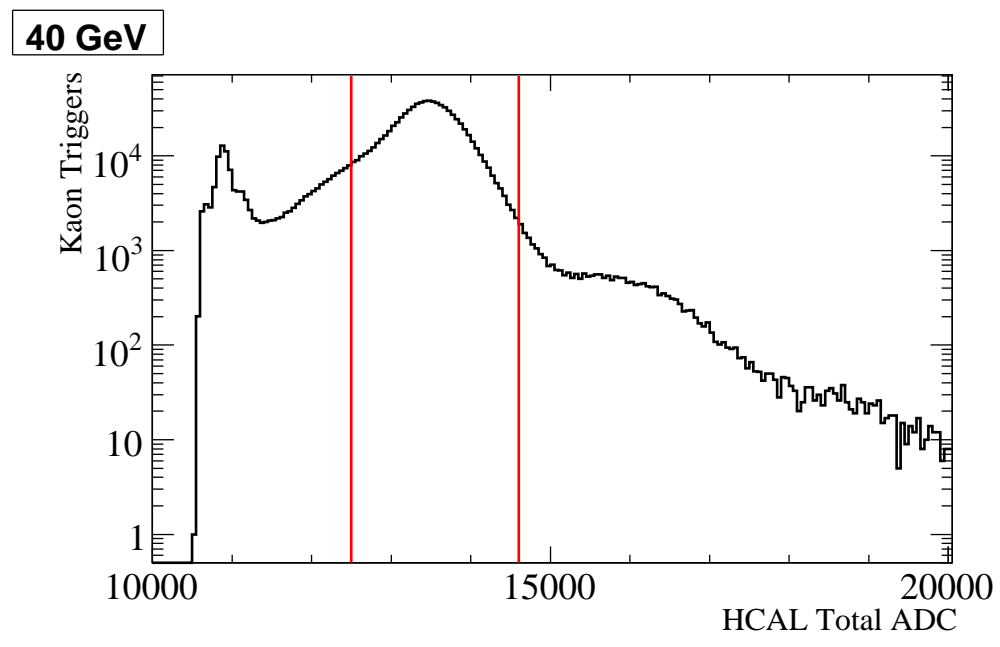

Figure 8.19: Hcal ADC sum for kaons from the $40 \mathrm{GeV}$ data set. Events between 12500 and 14600 were kept, as indicated by the vertical lines.

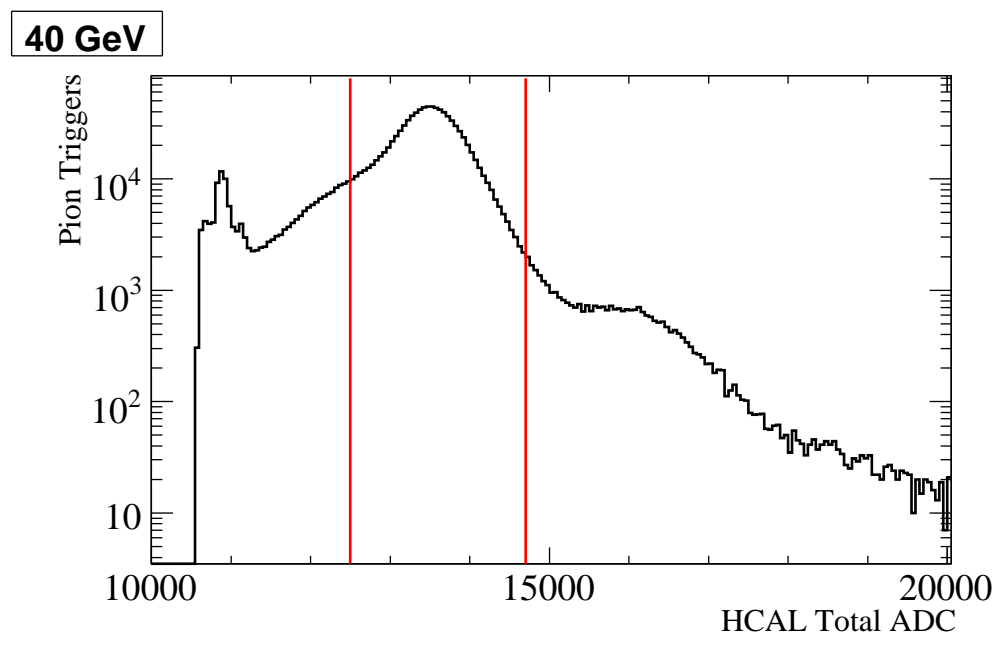

Figure 8.20: Hcal ADC sum for pions from the $40 \mathrm{GeV}$ data set. Events between 12500 and 14750 were kept, as indicated by the vertical lines. 


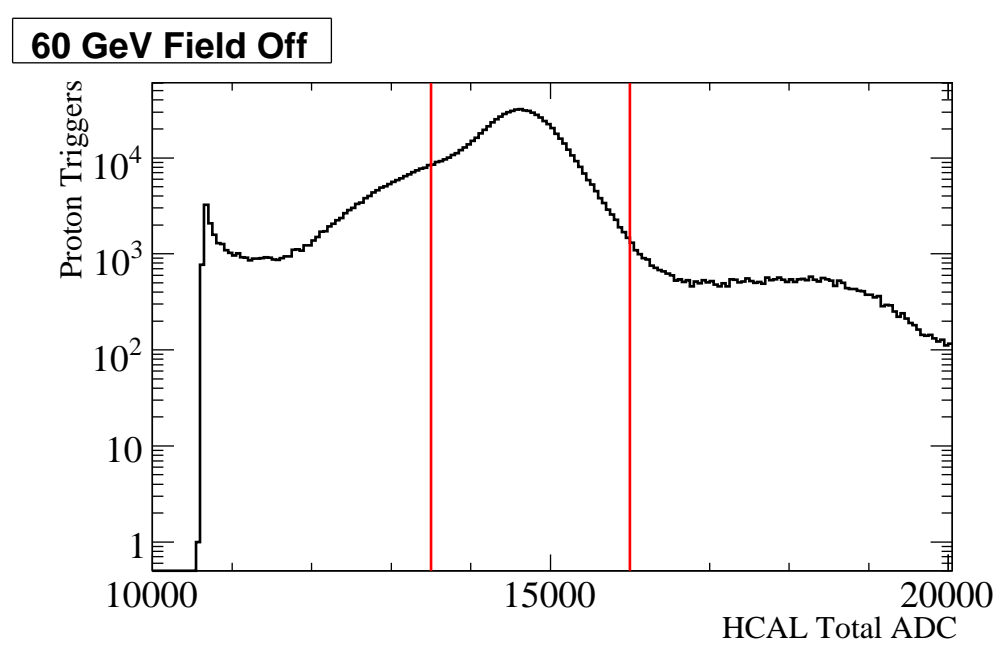

Figure 8.21: Hcal ADC sum for protons from the $60 \mathrm{GeV}$ field off data set. Events between 13500 and 16000 were kept, as indicated by the vertical lines.

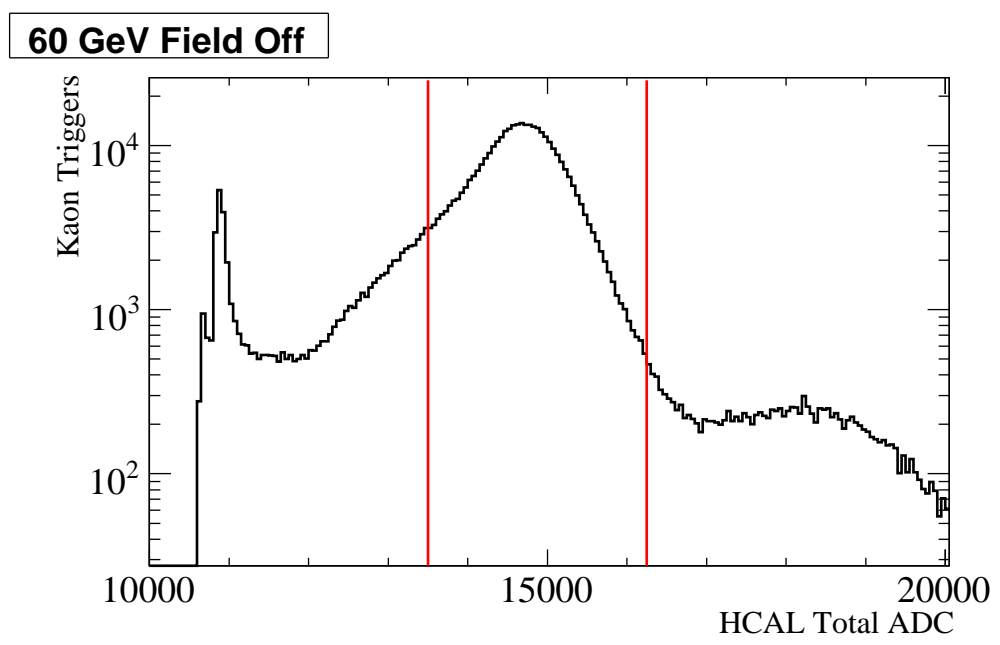

Figure 8.22: Hcal ADC sum for kaons from the $60 \mathrm{GeV}$ field off data set. Events between 13500 and 16250 were kept, as indicated by the vertical lines. 


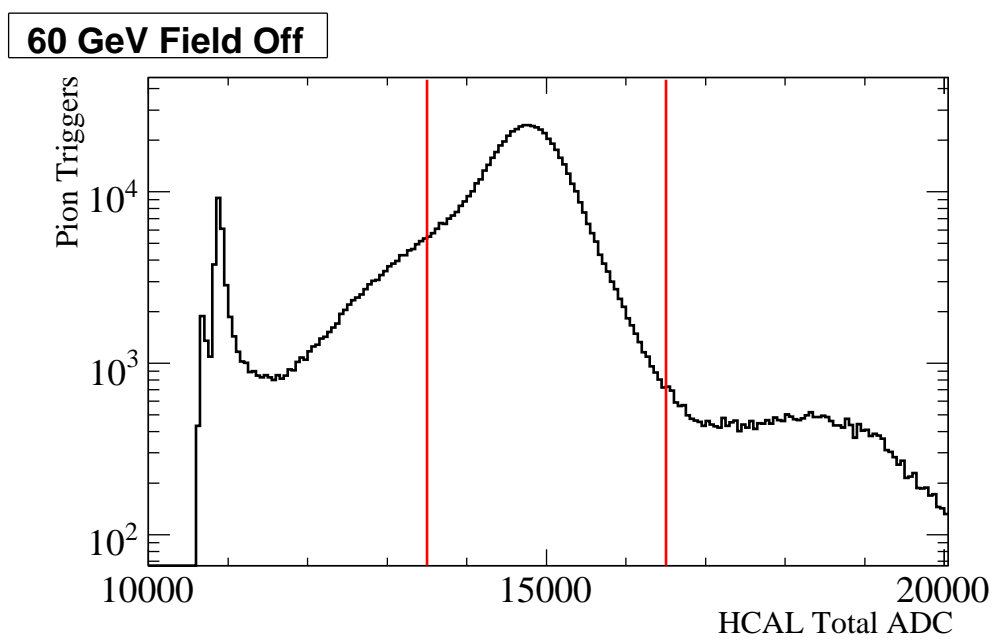

Figure 8.23: Hcal ADC sum for pions from the $60 \mathrm{GeV}$ field off data set. Events between 13500 and 16500 were kept, as indicated by the vertical lines.

most downstream chambers were used. Secondly, it was required to pass through both the upstream and downstream mylar windows of the RICH. Since this analysis requires straight through beam tracks, cuts were made on both the $x$ and $y$ slopes of the track segment. Finally, a $\chi^{2}$ requirement was imposed to ensure good quality fits.

Before determining the cut on track segment $\chi^{2}$, the slope distributions were considered. Reasonable, initial cuts on the slope in both $x$ and $y$ were made: $|\partial x / \partial z|<0.002,|\partial y / \partial z|<0.003 . \chi^{2}$ distributions for segments falling within this cut and outside it were compared. The large angle tracks proportionately more numerous at $\chi^{2}>2$ than are the small angle tracks, indicating that this is a good place to make the cut. 


\begin{tabular}{l|ccc}
\hline \hline Cut & Protons & Kaons & Pions \\
\hline Beam Trigger & 637451 & 769276 & 810765 \\
Clusters/Window & $607508(95.3 \%)$ & $737166(95.8 \%)$ & $784432(96.8 \%)$ \\
HCAL & $508994(79.8 \%)$ & $604409(78.6 \%)$ & $645687(79.6 \%)$ \\
$\chi^{2}$ & $364742(57.2 \%)$ & $432880(56.3 \%)$ & $463164(57.1 \%)$ \\
Slope & $328612(51.6 \%)$ & $396811(51.6 \%)$ & $423883(52.3 \%)$ \\
\hline Final Fractions & $28.6 \%$ & $34.5 \%$ & $36.9 \%$ \\
\hline \hline
\end{tabular}

Table 8.2: Summary of cuts for the $37 \mathrm{GeV}$ data set. The numbers are the remaining events after applying the indicated cut in the left hand column. The fraction remaining is show in parentheses. The final line shows the fraction of the total number of events after all cuts for each of $\pi / K / p$.

After applying the $\chi^{2}$ cut, a refined track slope was determined by examining $\pi, K$, and $p$ separately. For all three cases, track segments were chosen with $-0.002<\partial x / \partial z<0.0015$ and $-0.002<\partial y / \partial z<0.0015$.

The same track segments cuts were applied to each data set. Relevant plots for the $40 \mathrm{GeV} / c$ data set are shown in Figures 8.24-9.11.

After all cuts are applied, the amount was reduced to about $50 \%$ for all sets. The final total of 5.7 million events falls short of our 10 million event goal. The biggest losses were due to the HCAL and the track $\chi^{2}$ cuts which tend to discard about $20 \%$ each. The exception was the field-on set, which lost almost half the data due to the stricter requirement of wire clusters in only the three most downstream chambers. In total, this data set was reduced by nearly two-thirds once all cuts are applied.

Final $\pi / K / p$ fractions vary widely from set to set. In general, protons were the most abundant varying from $28 \%$ to $51 \%$ of the total. Kaons were 


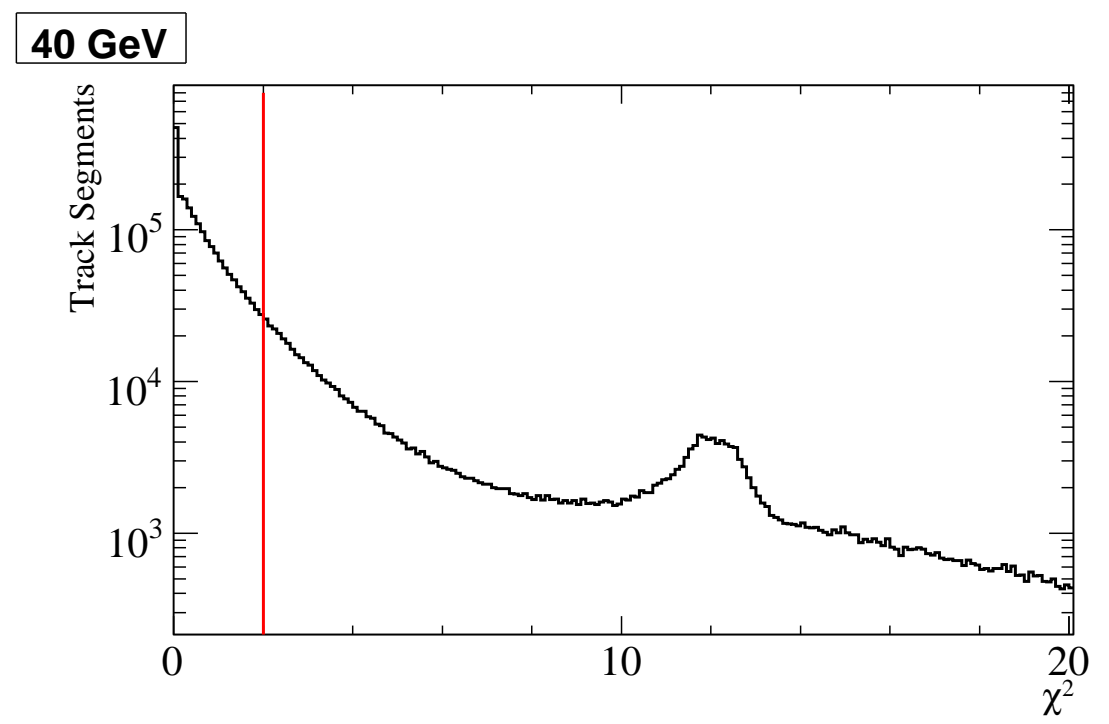

Figure 8.24: Track segment $\chi^{2}$ for the $40 \mathrm{GeV}$ data set. Tracks with $\chi^{2}>2$ are discarded, as indicated by the vertical line.

\begin{tabular}{l|ccc}
\hline \hline Cut & Protons & Kaons & Pions \\
\hline Beam Trigger & 1104688 & 1035826 & 1196312 \\
Clusters/Window & $1054646(95.5 \%)$ & $995139(96.1 \%)$ & $1158465(96.8 \%)$ \\
HCAL & $852356(77.2 \%)$ & $794148(76.7 \%)$ & $926629(77.4 \%)$ \\
$\chi^{2}$ & $633145(57.3 \%)$ & $591277(57.1 \%)$ & $693257(57.9 \%)$ \\
Slope & $571495(51.7 \%)$ & $542945(52.4 \%)$ & $636027(53.2 \%)$ \\
\hline Final Fractions & $32.6 \%$ & $31.0 \%$ & $36.3 \%$ \\
\hline \hline
\end{tabular}

Table 8.3: Summary of cuts for the $40 \mathrm{GeV}$ data set. The numbers are the remaining events after applying the indicated cut in the left hand column. The final line shows the fraction of the total number of events after all cuts for each of $\pi / K / p$. 

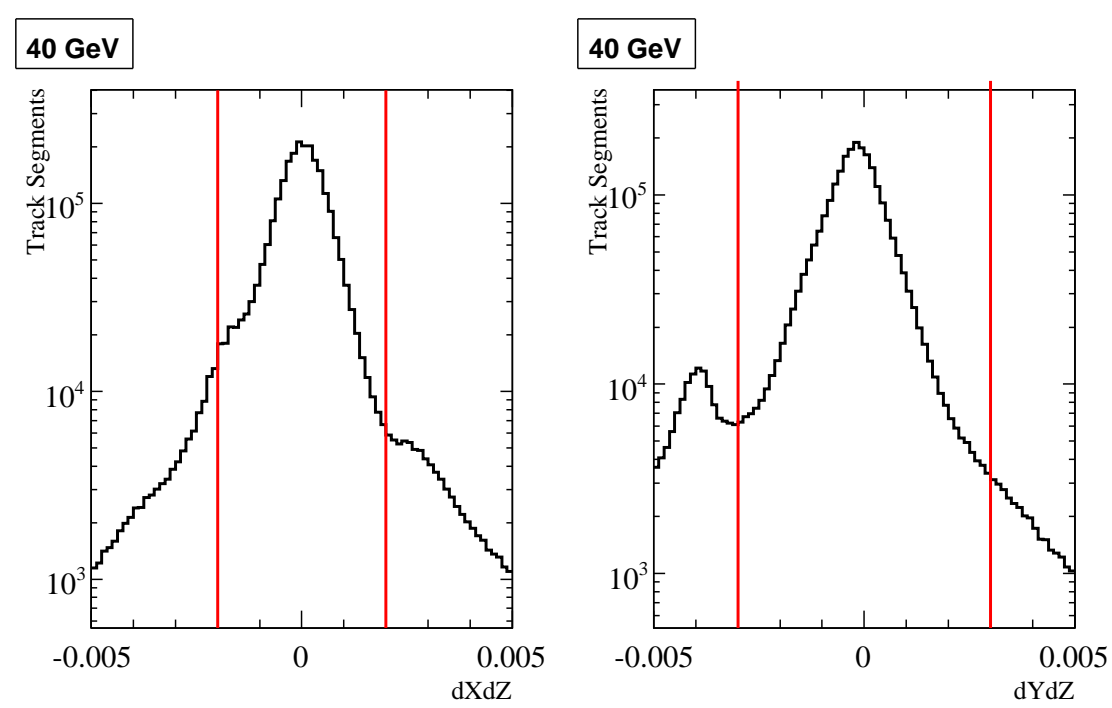

Figure 8.25: Track segment slope for the $40 \mathrm{GeV}$ data set. Initial loose cuts requiring $|\partial x / \partial z|<0.002$ and $|\partial y / \partial z|<0.003$ were made, as indicated by the vertical lines.

\begin{tabular}{l|ccc}
\hline \hline Cut & Protons & Kaons & Pions \\
\hline Beam Trigger & 413506 & 335265 & 338196 \\
Clusters/Window & $389292(94.1 \%)$ & $317941(94.8 \%)$ & $323790(95.7 \%)$ \\
HCAL & $331936(80.3 \%)$ & $252779(75.4 \%)$ & $259287(76.7 \%)$ \\
$\chi^{2}$ & $234336(56.7 \%)$ & $180102(53.7 \%)$ & $184448(54.5 \%)$ \\
Slope & $212901(51.5 \%)$ & $166147(49.6 \%)$ & $170618(50.4 \%)$ \\
\hline Final Fractions & $38.7 \%$ & $30.2 \%$ & $31.0 \%$ \\
\hline \hline
\end{tabular}

Table 8.4: Summary of cuts for the $42 \mathrm{GeV}$ data set. The numbers are the remaining events after applying the indicated cut in the left hand column. The final line shows the fraction of the total number of events after all cuts for each of $\pi / K / p$. 


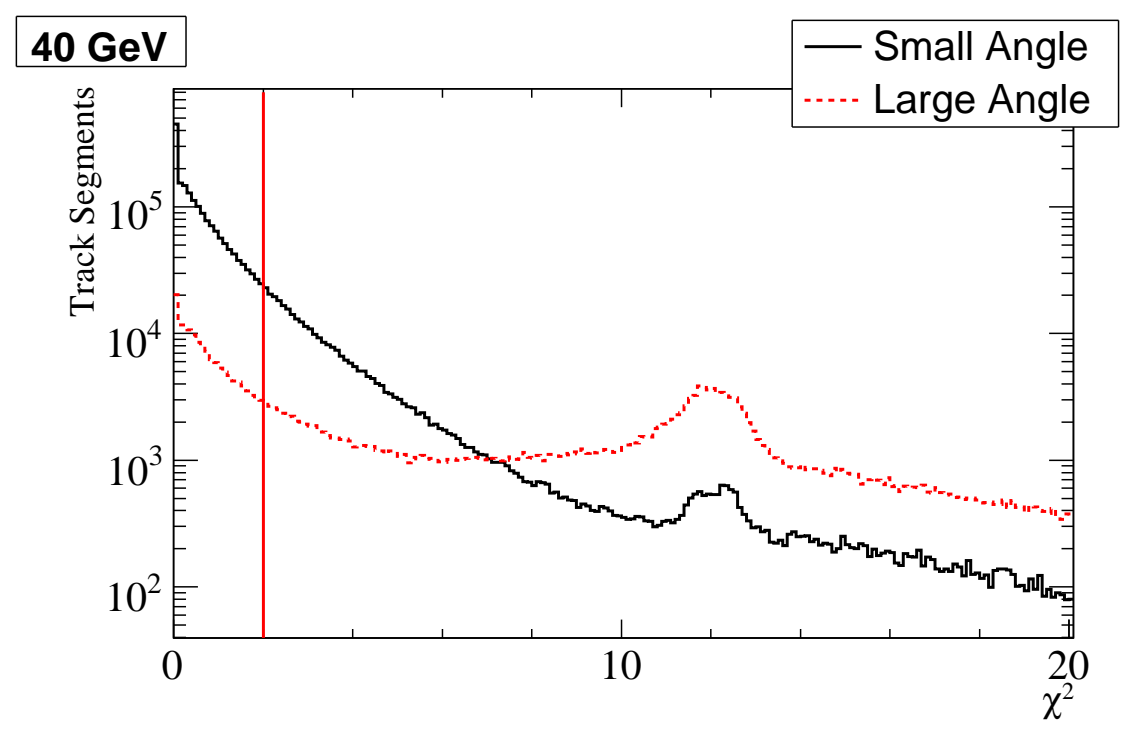

Figure 8.26: $\chi^{2}$ comparison between large angle and small angle track segments for the $40 \mathrm{GeV}$ data set. Tracks with $\chi^{2}>2$ are discarded, as indicated by the vertical line.

\begin{tabular}{l|ccc}
\hline \hline Cut & Protons & Kaons & Pions \\
\hline Beam Trigger & 178353 & 124219 & 160514 \\
Clusters/Window & $153783(86.2 \%)$ & $107174(86.3 \%)$ & $139904(87.2 \%)$ \\
HCAL & $110796(62.1 \%)$ & $75898(61.1 \%)$ & $97710(60.9 \%)$ \\
$\chi^{2}$ & $86380(48.4 \%)$ & $59216(47.7 \%)$ & $76813(47.9 \%)$ \\
Slope & $78852(44.2 \%)$ & $54894(44.2 \%)$ & $71104(44.2 \%)$ \\
\hline Final Fractions & $38.5 \%$ & $26.8 \%$ & $34.7 \%$ \\
\hline \hline
\end{tabular}

Table 8.5: Summary of cuts for the $56 \mathrm{GeV}$ data set. The numbers are the remaining events after applying the indicated cut in the left hand column. The final line shows the fraction of the total number of events after all cuts for each of $\pi / K / p$. 

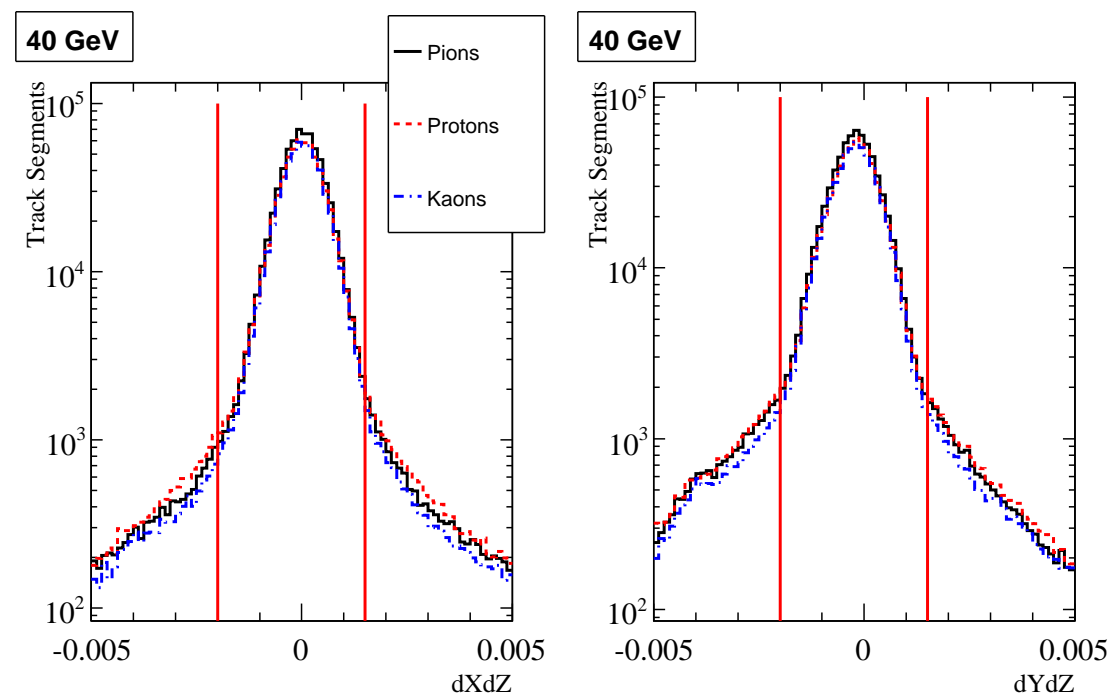

Figure 8.27: Track segment slopes for the $40 \mathrm{GeV}$ data set. Horizontal slope is on the left and vertical slope is on the right. Distributions are very similar for $\pi, K, p$, so the same cuts can be applied. The final track slope requirements were $-0.002<\partial x / \partial z<0.0015$ and $-0.002<\partial y / \partial z<0.0015$, as indicated by the vertical lines.

\begin{tabular}{l|ccc}
\hline \hline Cut & Protons & Kaons & Pions \\
\hline Beam Trigger & 1190451 & 524624 & 949961 \\
Clusters/Window & $975670(82.0 \%)$ & $443873(84.6 \%)$ & $801698(84.3 \%)$ \\
HCAL & $769563(64.6 \%)$ & $352258(67.1 \%)$ & $632662(66.6 \%)$ \\
$\chi^{2}$ & $637478(53.5 \%)$ & $287277(54.8 \%)$ & $520290(54.8 \%)$ \\
Slope & $585720(49.2 \%)$ & $267253(50.9 \%)$ & $483385(50.9 \%)$ \\
\hline Final Fractions & $43.8 \%$ & $20.0 \%$ & $36.2 \%$ \\
\hline \hline
\end{tabular}

Table 8.6: Summary of cuts for the $60 \mathrm{GeV}$ field off data set. The numbers are the remaining events after applying the indicated cut in the left hand column. The final line shows the fraction of the total number of events after all cuts for each of $\pi / K / p$. 


\begin{tabular}{l|ccc}
\hline \hline Cut & Protons & Kaons & Pions \\
\hline Beam Trigger & 900605 & 384562 & 688882 \\
Clusters/Window & $481385(53.5 \%)$ & $201953(52.5 \%)$ & $374328(54.3 \%)$ \\
HCAL & $381315(42.3 \%)$ & $157165(40.9 \%)$ & $291522(42.3 \%)$ \\
$\chi^{2}$ & $323587(35.9 \%)$ & $132372(34.4 \%)$ & $248079(36.0 \%)$ \\
Slope & $290838(32.3 \%)$ & $120382(31.2 \%)$ & $225546(32.7 \%)$ \\
\hline Final Fractions & $45.7 \%$ & $18.9 \%$ & $35.4 \%$ \\
\hline \hline
\end{tabular}

Table 8.7: Summary of cuts for the $60 \mathrm{GeV}$ field on data set. The numbers are the remaining events after applying the indicated cut in the left hand column. The final line shows the fraction of the total number of events after all cuts for each of $\pi / K / p$.

\begin{tabular}{l|ccc}
\hline \hline Cut & Protons & Kaons & Pions \\
\hline Beam Trigger & 132212 & 48135 & 75010 \\
Clusters/Window & $126144(95.4 \%)$ & $46093(95.8 \%)$ & $72238(96.3 \%)$ \\
HCAL & $92697(70.1 \%)$ & $33965(70.6 \%)$ & $53390(71.2 \%)$ \\
$\chi^{2}$ & $74864(56.6 \%)$ & $27462(57.1 \%)$ & $43470(58.0 \%)$ \\
Slope & $68187(51.6 \%)$ & $25364(52.7 \%)$ & $40083(53.4 \%)$ \\
\hline Final Fractions & $51.0 \%$ & $19.0 \%$ & $30.0 \%$ \\
\hline \hline
\end{tabular}

Table 8.8: Summary of cuts for the $63 \mathrm{GeV}$ data set. The numbers are the remaining events after applying the indicated cut in the left hand column. The final line shows the fraction of the total number of events after all cuts for each of $\pi / K / p$. 


\begin{tabular}{l|cccc}
\hline \hline Data Set & Protons & Kaons & Pions & Total \\
\hline $37 \mathrm{GeV}$ & 328612 & 396811 & 423883 & 1149306 \\
$40 \mathrm{GeV}$ & 571495 & 542945 & 636027 & 1750467 \\
$42 \mathrm{GeV}$ & 212901 & 166147 & 170618 & 549666 \\
$56 \mathrm{GeV}$ & 78852 & 54894 & 71104 & 204850 \\
$60 \mathrm{GeV}$ Field Off & 585720 & 267253 & 483385 & 1336358 \\
$60 \mathrm{GeV}$ Field On & 290838 & 120382 & 225546 & 636766 \\
$63 \mathrm{GeV}$ & 68187 & 25364 & 40083 & 133634 \\
\hline Totals & 2136605 & 1573796 & 2050646 & 5761047 \\
\hline \hline
\end{tabular}

Table 8.9: Summary of data totals for each data set after applying cuts.

in the minority, varying from $19 \%$ to $34 \%$. This differs significantly from the optimal fractions of $35 \%$ pions, $45 \%$ kaons, and $20 \%$ protons for which we aimed. 


\section{Chapter 9}

\section{Alignment}

A properly aligned detector is a necessity in any experiment. To ensure reliable prediction of ring centers, all the chambers and the RICH mirrors must be aligned.

\subsection{Chamber Alignment}

Chamber plane positions initially used in reconstructed were from a survey of the experiment hall. Agreement with the data was within a few millimeters. Even this small amount of disagreement had a significant effect on predicted track position as seen in the top plot of Figure 9.1. For example, a 120 $\mathrm{GeV} / c$ track would deviate from a straight line by $1.8 \mathrm{~cm}$ over the length of the experiment. This means predicted ring center would be off by about 1.3 times the PMT spacing in the RICH, which would significantly affect 
predictions of light distribution.

First beam chamber planes were aligned separately. Since most events have a good beam track, high statistics were available for beam chamber alignment. The remaining six chambers where then aligned separately from the beam tracks, allowing relative chamber rotations to be determined. This could not be done with beam chambers because the lever arm was too short. Last all nine chambers were aligned to each other ensuring consistency.

Selecting good track fits is essential. When aligning drift and wire chambers, only tracks with at least two of four planes in each chamber were selected. This requirement cut out incorrectly reconstructed tracks while still leaving a sufficiently large data set. Chamber efficiency was too low to require hits in all chamber planes. Any planes with two hit wires were discarded to limit noise and beam pileup.

The following algorithm was used to align the chamber planes: for each wire in each track, calculate residual when the wire is excluded from the fit; calculate mean of residuals for each wire plane using all tracks; shift wire plane by 0.3 of the mean of residuals; if there are multiple tracks, exclude those with any residuals greater than 1.5 wire spacings plus the absolute value of the mean; repeat until the largest mean of residuals is less than $1 \%$ of the wire spacing.

The final step was to correct $z$ positions and $z$ axis rotations in the spectrometer geometry [25]. The algorithm is similar to how the plane offsets were found. The sum of $\chi^{2}$ for all tracks is minimized by varying the $z$ 


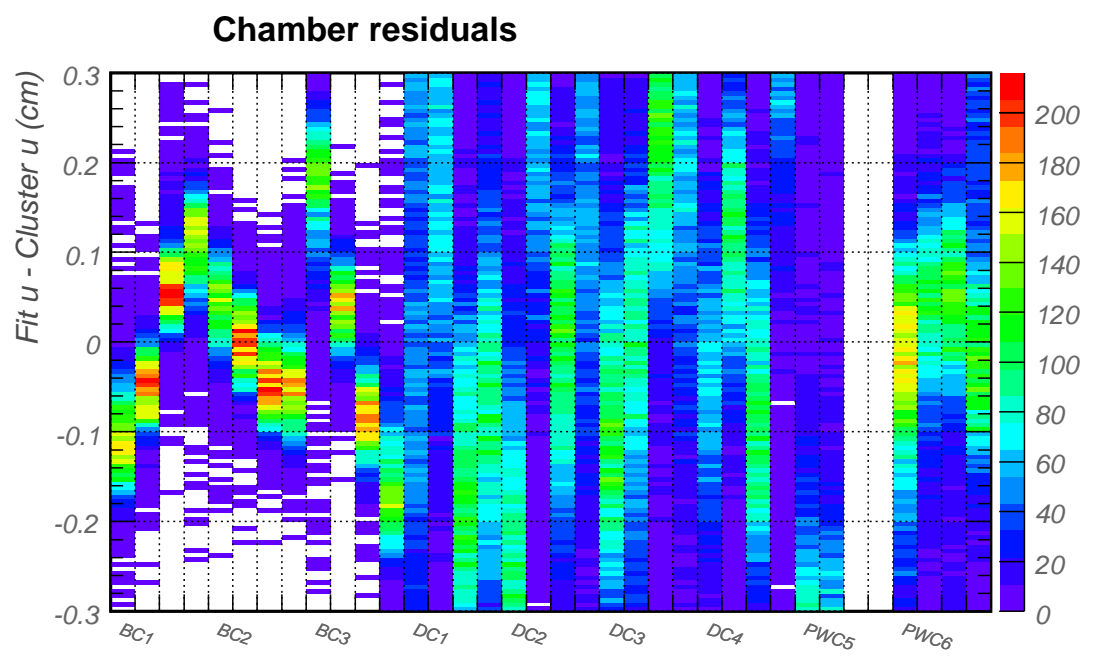

Chamber residuals, post-alignment

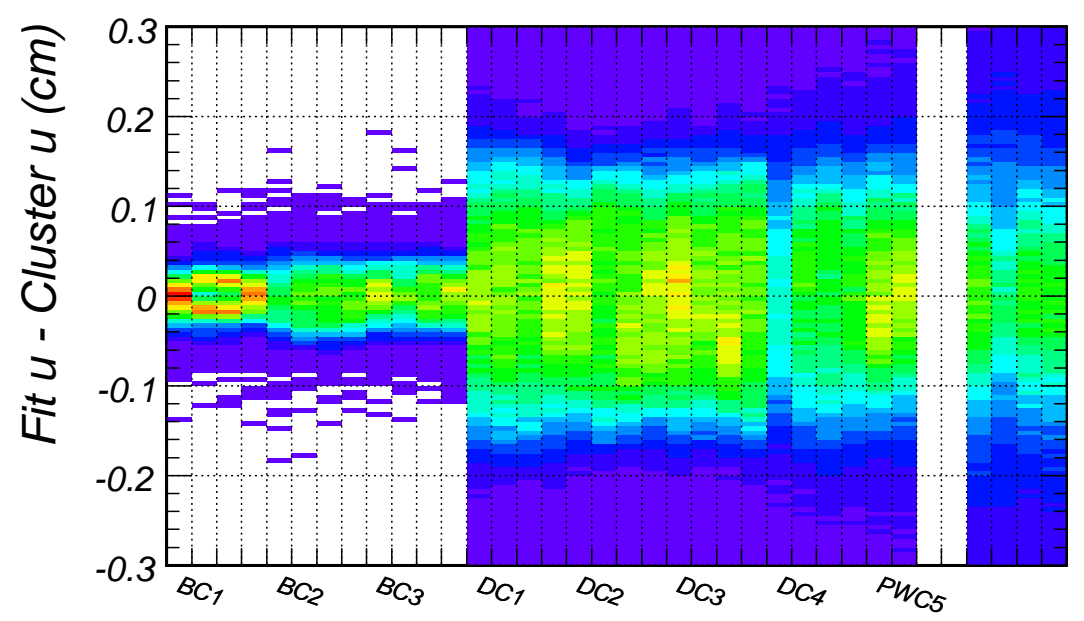

Figure 9.1: Top: Chamber residuals before alignment. Bottom: Chamber residuals after alignment. Chamber planes are on the horizontal axis. The vertical axis is the residuals from track fits. Alignment is good to within less than one wire spacing. 
position of the chambers and their rotations about the $z$ axis.

Even after applying these corrections to the geometry, DC3 still had a $z$ axis rotation of nearly $1 \mathrm{mrad}$ (see Figure 9.2). This correction was applied and the $z$ alignment algorithm re-run. Figure 9.3 shows that the algorithm suggests moving DC123 by $5 \mathrm{~mm}, 3 \mathrm{~mm}$, and $2 \mathrm{~mm}$ respectively, resulting in a systematic error on position of less than a tenth of a wire spacing, and could be due to effects of the Earth's magnetic field or $x$ and $y$ axis rotations which are not taken into account. These corrections were not applied. This has no effect on the charge kaon mass analysis since tracking relies mainly on the three most downstream chambers.

\subsection{RICH Mirror Alignment}

Mirror alignment was done on a run-by-run basis. At least 20,000 events were required. Shorter runs were combined together to ensure enough statistics.

The idea is to adjust the predicted ring center obtained by reflecting the track off a given mirror to the center found by the ring fitting algorithm. For this to work, tubes seeing light from only one mirror need to be used. Rings, however, see light from multiple mirrors. Each tube needed to be assigned to a particular mirror if possible.

This was accomplished using a brute force method. For each PMT hit, the Cherenkov and azimuthal angles of the center were estimated by reflecting the track off the mirror array to predict the ring center. For nine points 

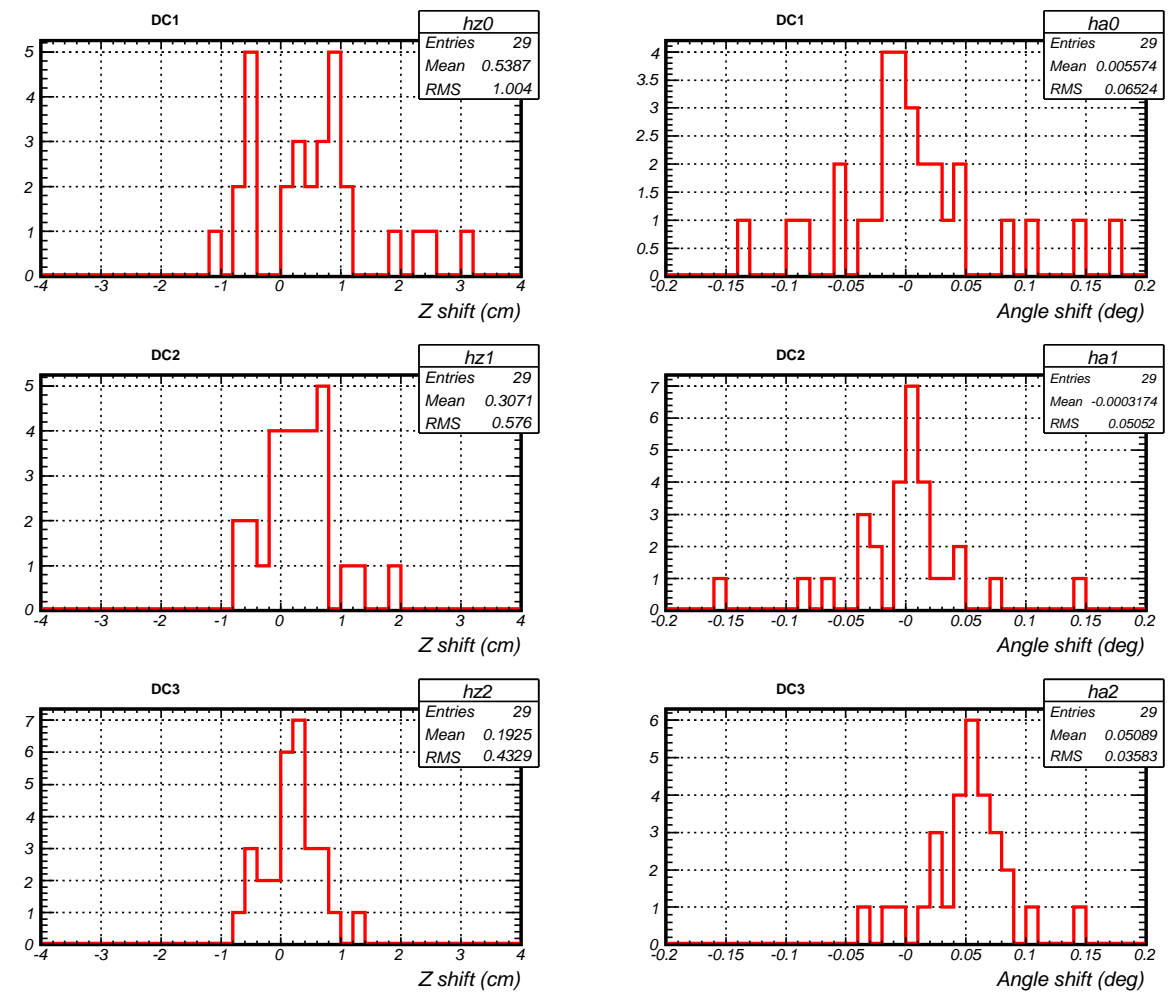

Figure 9.2: DC124 $z$-alignment with field-off runs. DC3 is still systematically rotated by $z$-alignment algorithm. 

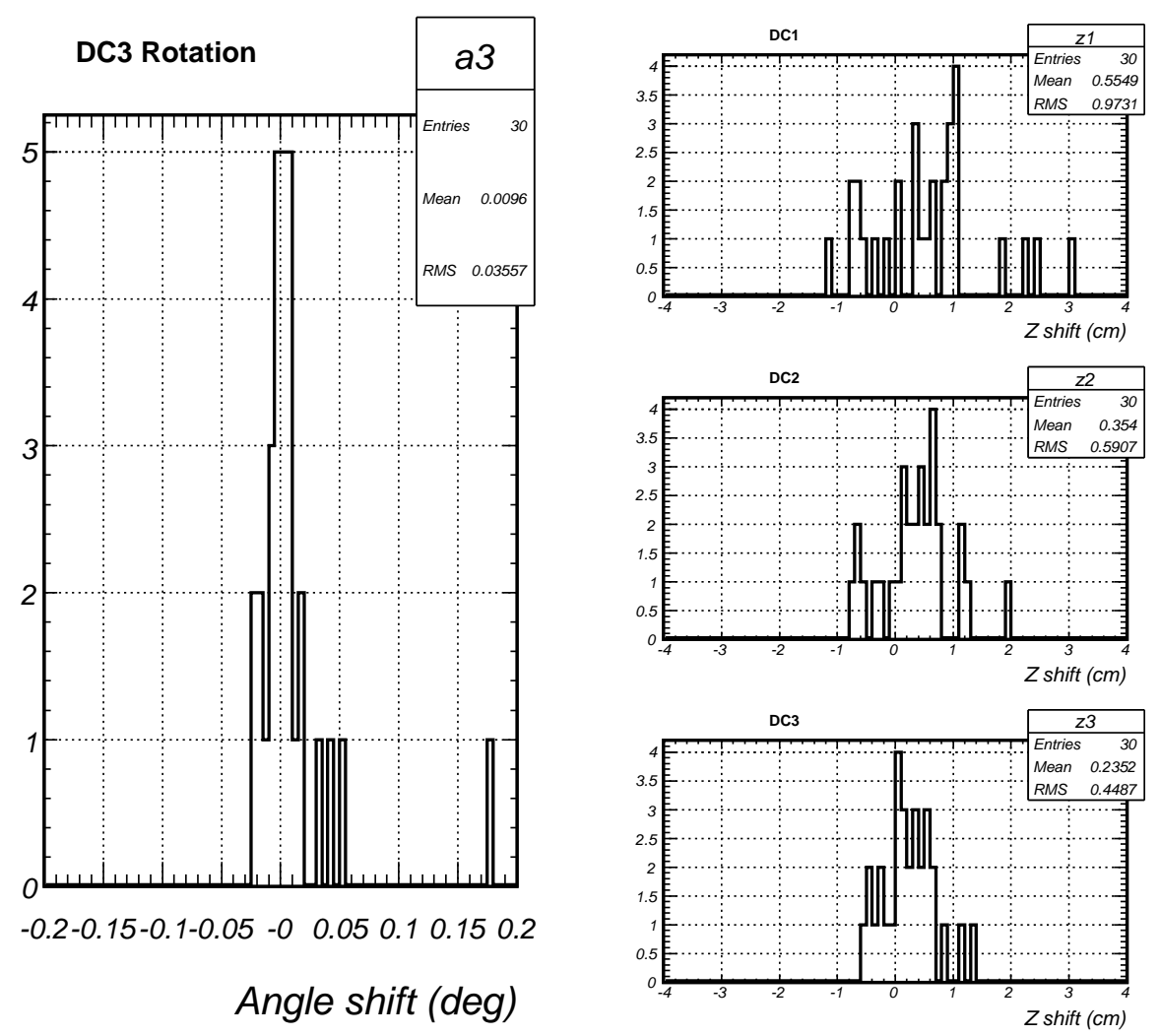

Figure 9.3: DC123 z-alignment without DC3 rotation. Chamber positions are still off by a few $\mathrm{mm}$. Systematic errors on track position are less than one wire spacing. 
distributed over the PMT face, rays were traced from 25 evenly spaced points along the track's path through the RICH at the appropriate angles to the mirror array. The mirror hit by each ray was found, and the fraction of light each tube sees from each mirror was estimated. The cutout section in mirror 8 was taken into account. This was averaged over all events passing the cuts discussed in Chapter 8 for each set individually.

Most tubes see the vast majority of light ( $>90 \%)$ from one of the two central mirrors, 8 and 9 . This was not surprising as beam particles pass through the center of the RICH near the boundary between mirrors 8 and 9. In fact, rings can essentially be split vertically in half with tubes on one side assigned to mirror 8 and tubes on the other assigned to mirror 9. The only exceptions were PMTs near the top and bottom of proton rings which see some light from mirrors 3 and 14, which are directly above and below the two central mirrors respectively. These tubes were left out of the alignment.

Offsets to vertical and horizontal PMT position were added to the ring fitting algorithm for mirrors 8 and 9 separately. The fitter was ran twice on each event, once allowing mirror 8 hits to be moved and once allowing mirror 9 hits to be moved. Thus mirror 8 was aligned to mirror 9 and vice versa.

Two cuts were made to ensure good ring fits: at least 10 PMT hits for proton rings, at least 15 hits for kaon and pion rings (Figure 9.4), and a reasonable ring radius (Figure 9.5). The examples given are for a $60 \mathrm{GeV} / c$ field off run. In this case, a ring radius between $22 \mathrm{~cm}$ and $33 \mathrm{~cm}$ was required. Normally the kaon and pion peaks are better separated than what 


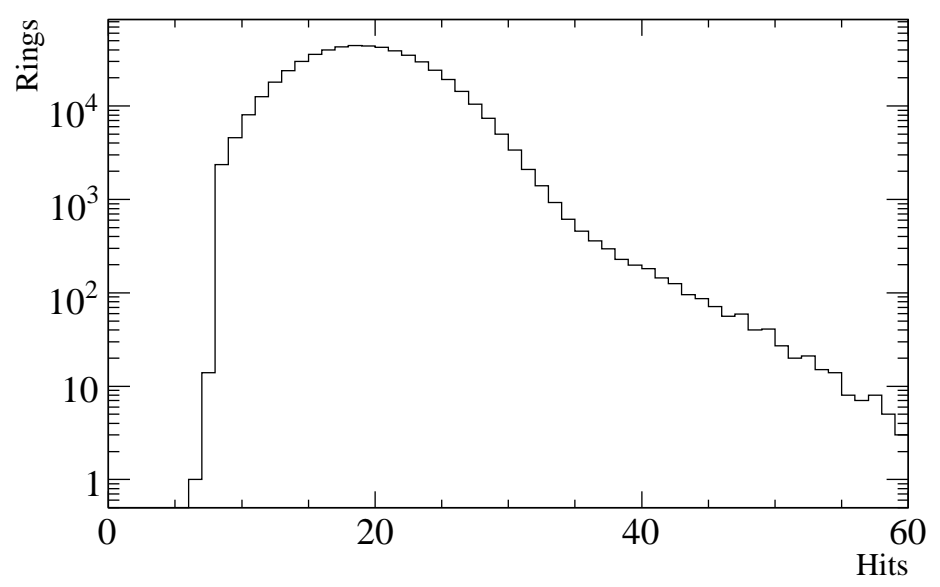

Figure 9.4: Number of PMT hits in ring fits. To be used in mirror alignment, at least 10 hits were required for proton rings and 15 hits for both kaon and pion rings.

is seen here. The additional degrees of freedom introduced by the position offsets lowered the radius resolution causing the two peaks to overlap more.

As expected, the tube position offsets for mirrors 8 and 9 were equal in magnitude and opposite and direction. Horizontal offsets were typically about $0.4 \mathrm{~cm}$, and vertical offsets typically $0.08 \mathrm{~cm}$.

Ring fit results were compared to center predictions based on mirrors 8 and 9 and differences in horizontal and vertical position are plotted in Figures 9.7-9.10. The shapes of the distributions are not particularly Gaussian, though reasonable fits were obtained by considering only regions near the peaks. The mean values from the fits were used as the alignment constants.

Final predicted position distributions after alignment are shown in Figure 


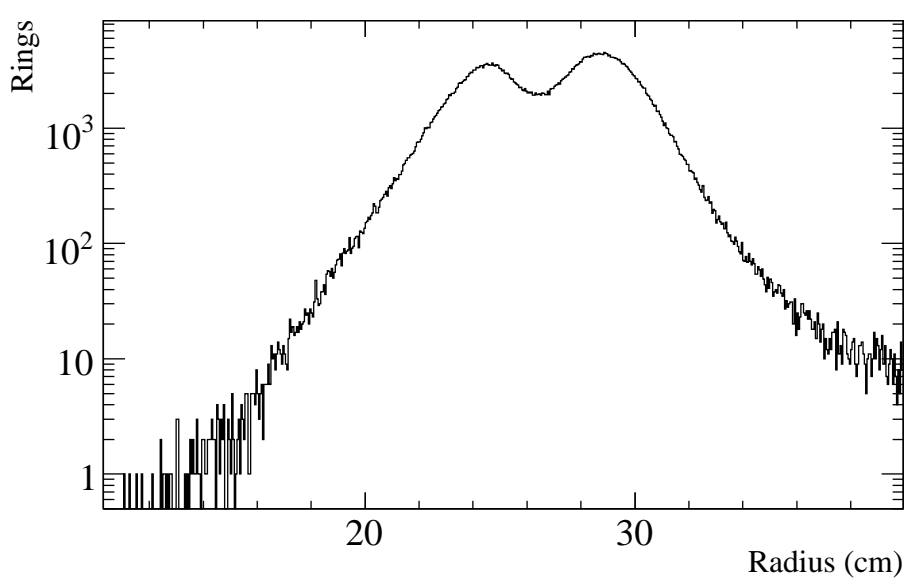

Figure 9.5: Fitted ring radius. Rings with radii between $22 \mathrm{~cm}$ and $33 \mathrm{~cm}$ were used in mirror alignment

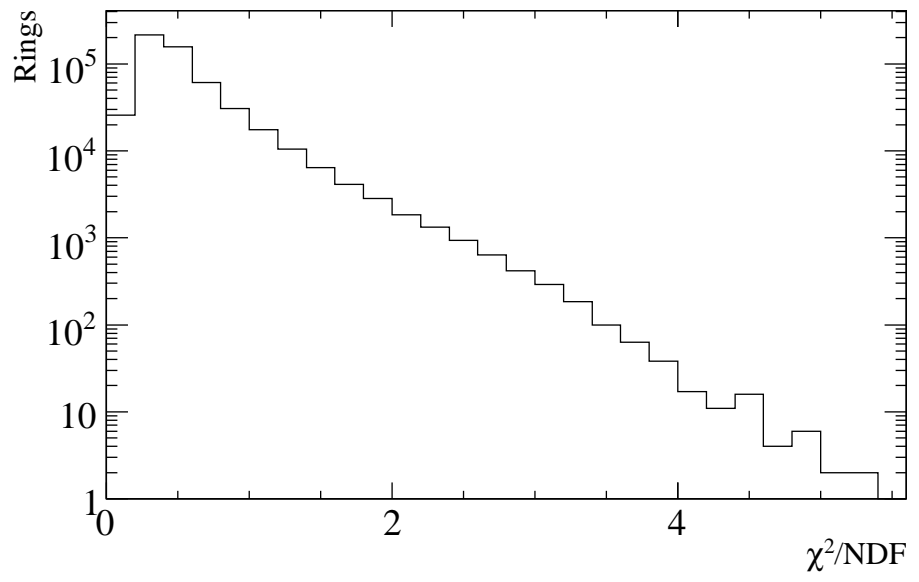

Figure 9.6: $\chi^{2}$ per degree of freedom for ring fits. No cut was made on ring $\chi^{2}$. 


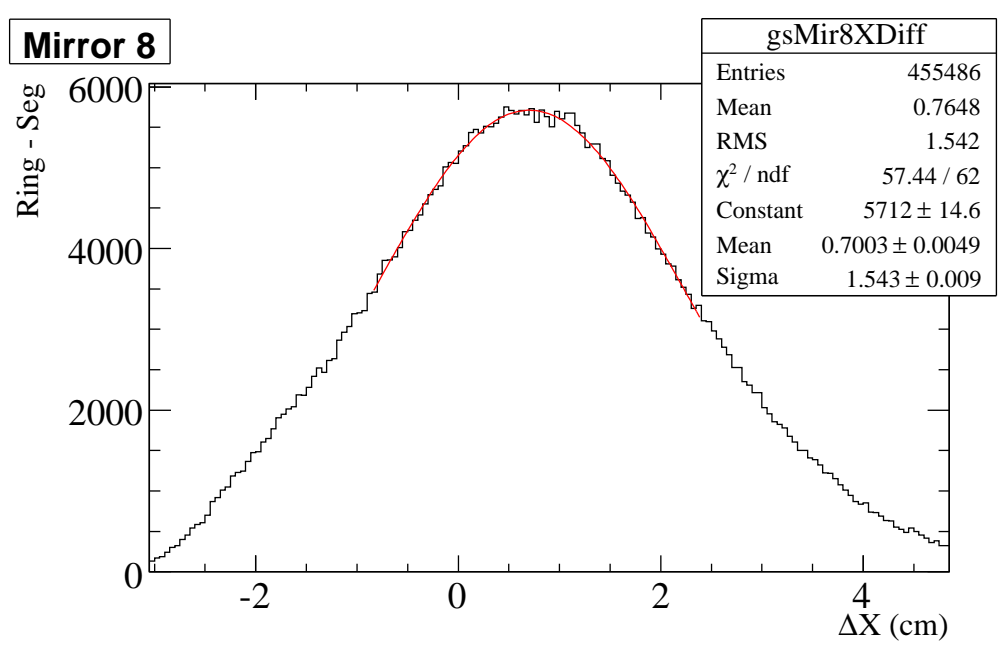

Figure 9.7: Difference between horizontal position of fitted ring center and predicted track center for mirror 8 . The curve is a Gaussian fit to central region to find the peak.

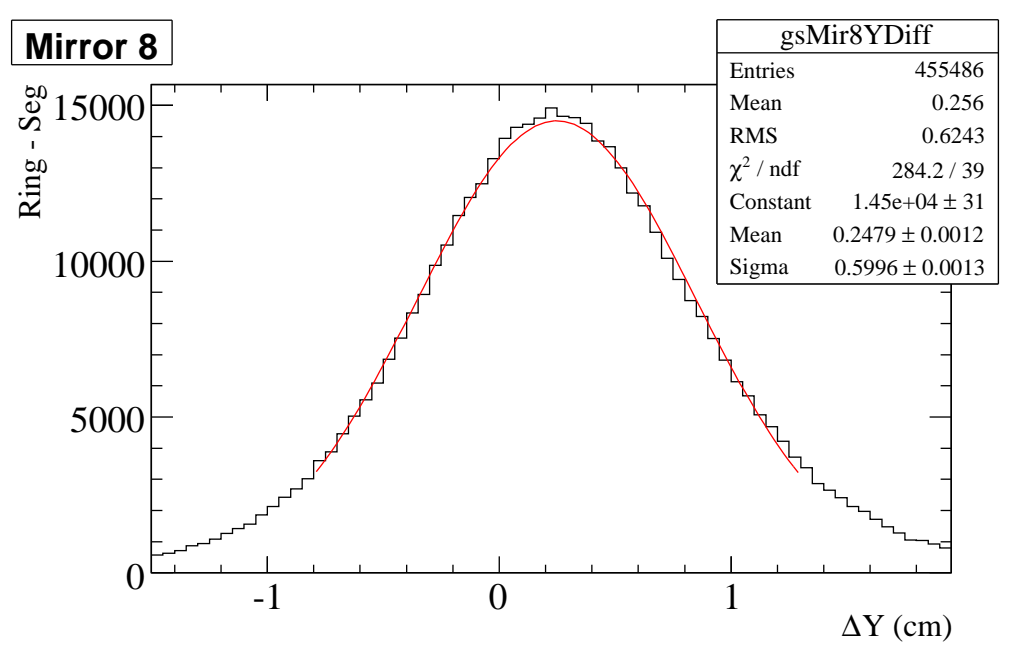

Figure 9.8: Difference between vertical position of fitted ring center and predicted track center for mirror 8 . The curve is a Gaussian fit to central region to find the peak. 


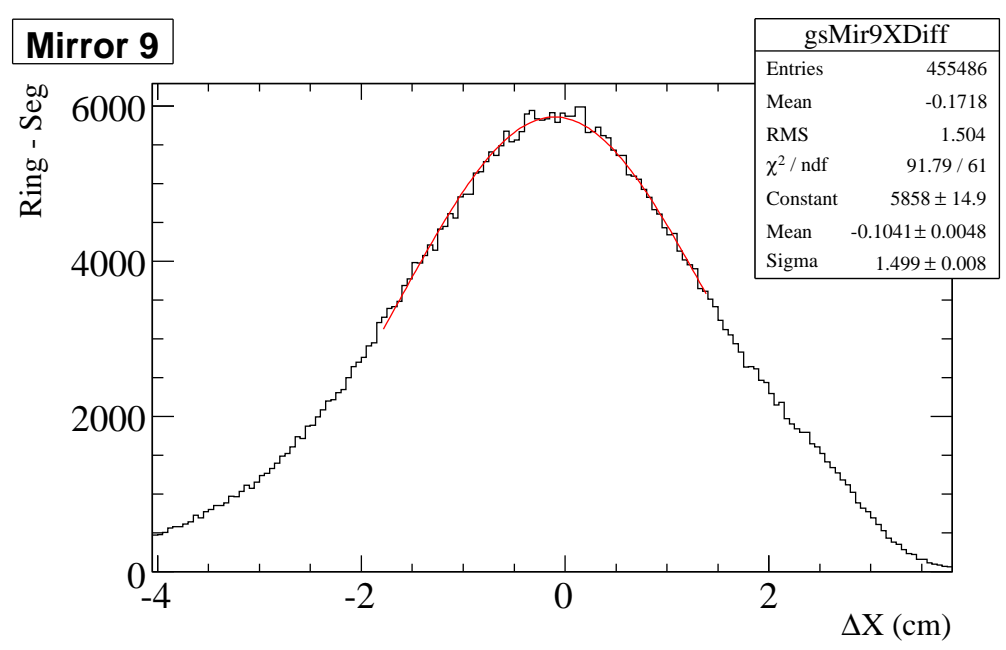

Figure 9.9: Difference between horizontal position of fitted ring center and predicted track center for mirror 9. The curve is a Gaussian fit to central region to find the peak.

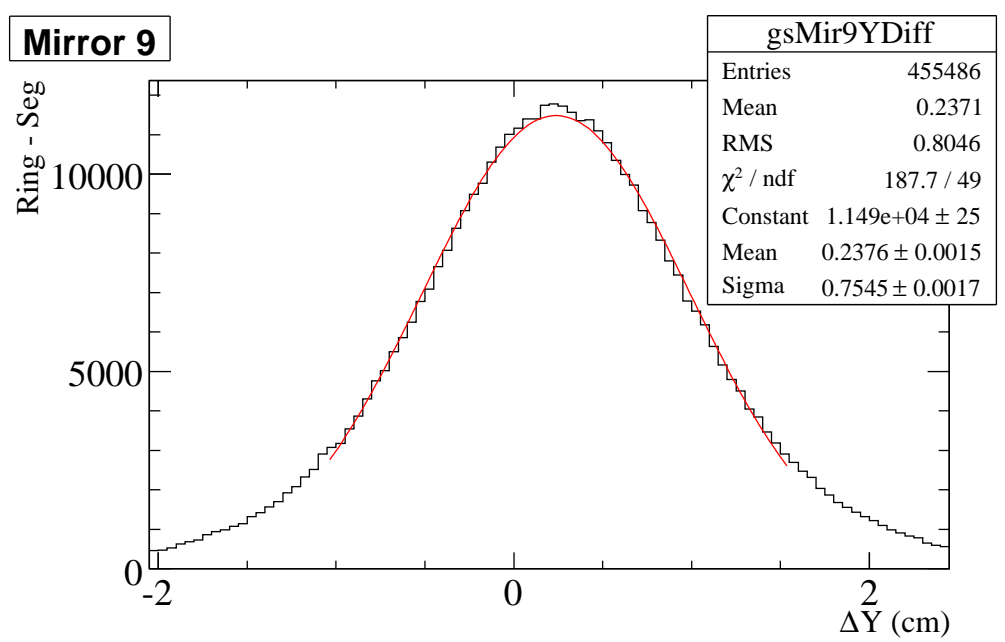

Figure 9.10: Difference between vertical position of fitted ring center and predicted track center for mirror 9. The curve is a Gaussian fit to central region to find the peak. 

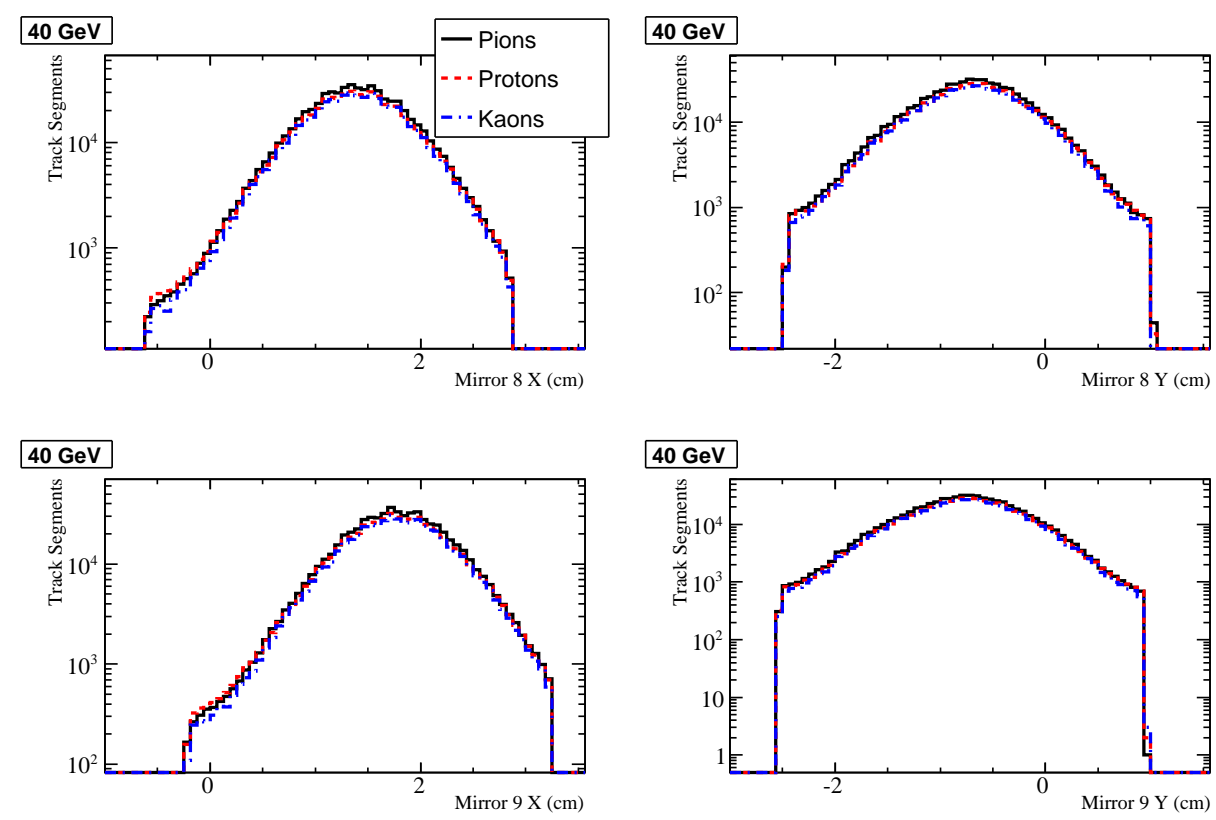

Figure 9.11: Projected ring center for the $40 \mathrm{GeV}$ data set. The top row is mirror 8 and the bottom row is mirror 9 , with horizontal position on the right and vertical position on the left. Distributions for $\pi, K$, and $p$ are superimposed and are nearly identical.

9.11 for the $40 \mathrm{GeV}$ data set. The distributions for all three particle types are nearly identical. The relationship between mirror 8 predicted position and mirror 9 predicted position is shown in Figure 9.12. Both horizontal and vertical position show a linear dependence. 

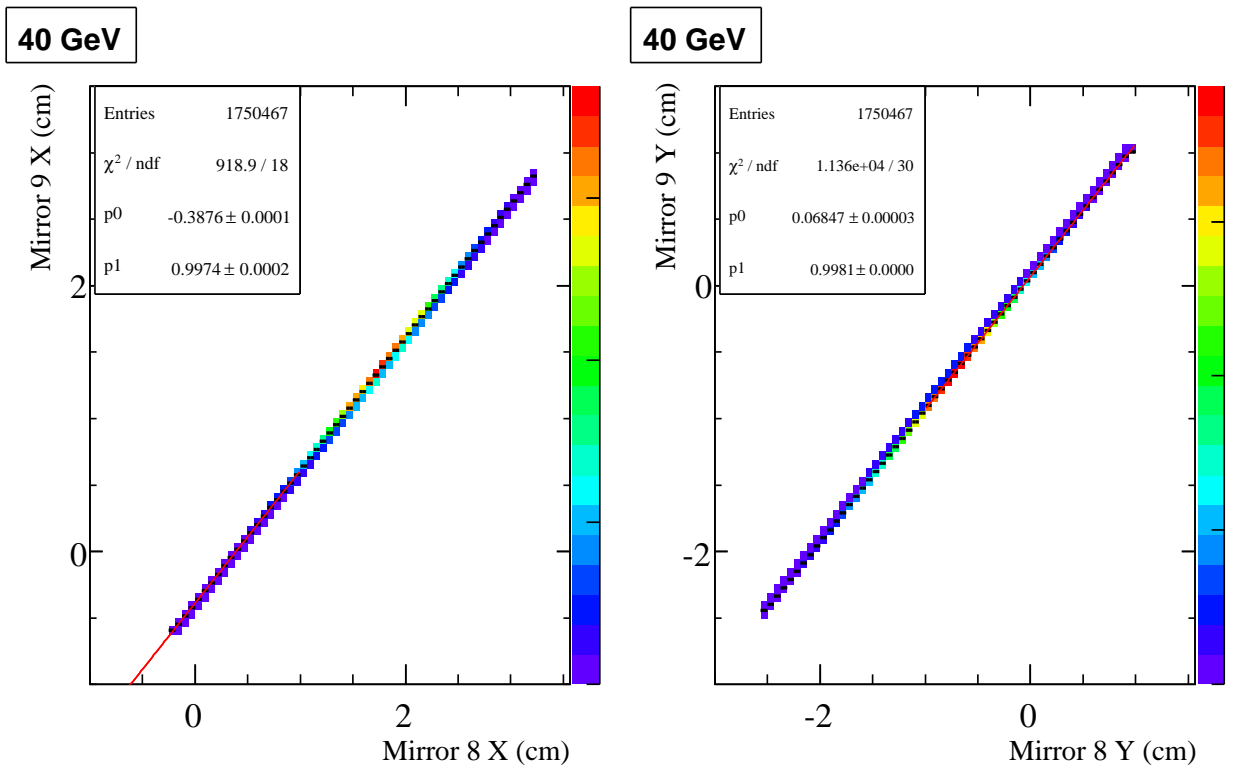

Figure 9.12: Predicted ring center for mirror 9 vs mirror 8. Horizontal position is on the left and vertical position is on the right. Linear fits are superimposed. 


\section{Chapter 10}

\section{Data Analysis}

\subsection{Light Yield in More Detail}

So far we have been considering the pattern of Cherenkov light in the RICH as a ring with a single radius or angle. This is an oversimplification since index of refraction is a function of wavelength, which means light is distributed over a range of angles. Therefore the full light prediction is necessary to make this measurement correctly. This is done by integrating Eqn. 5.2 over path length through the RICH and the PMT wavelength range of sensitivity while including the functional dependence of refractive index on wavelength, shown in Figure 10.1. Both the data and parameterization are taken from [6].

There are several remaining factors which must be accounted for to arrive at a correct prediction for the light yield. Mirror and cone reflectance, 


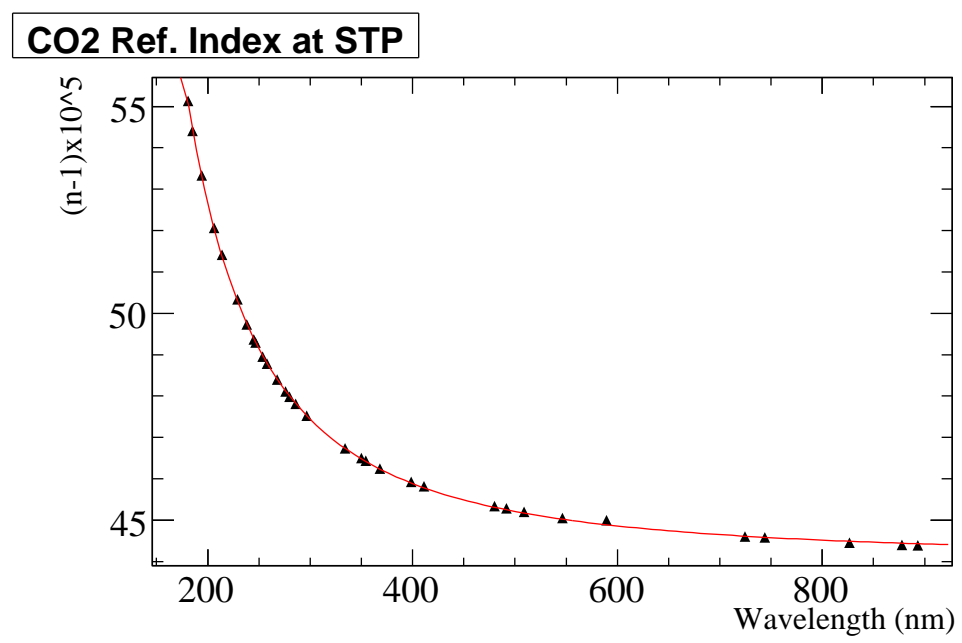

Figure 10.1: Refractive index of carbon dioxide as a function of wavelength at STP. The black circles are data points and red line is parameterization to the data. Both are taken from [6].

quartz window transmittance, and absorption by $\mathrm{CO}_{2}$ reduce the overall yield, while multiple scattering of the particle as it passes through the RICH and scattering of the light spread it out. Finally, the quantum and collection efficiencies convert the amount of predicted light into an expected number of photoelectrons.

Each PMT has a geometric acceptance for light at a particular angle. For a given wavelength which corresponds to a particular Cherenkov angle, and thus ring radius, this is simply the fraction of the arc which overlaps the area of the tube face, as shown in Figure 10.7. Given that the point $I$ lies on both circles, the distances $x$ and $y$ can be found. $\cos (\phi)=y / R_{c}$, and the arc 


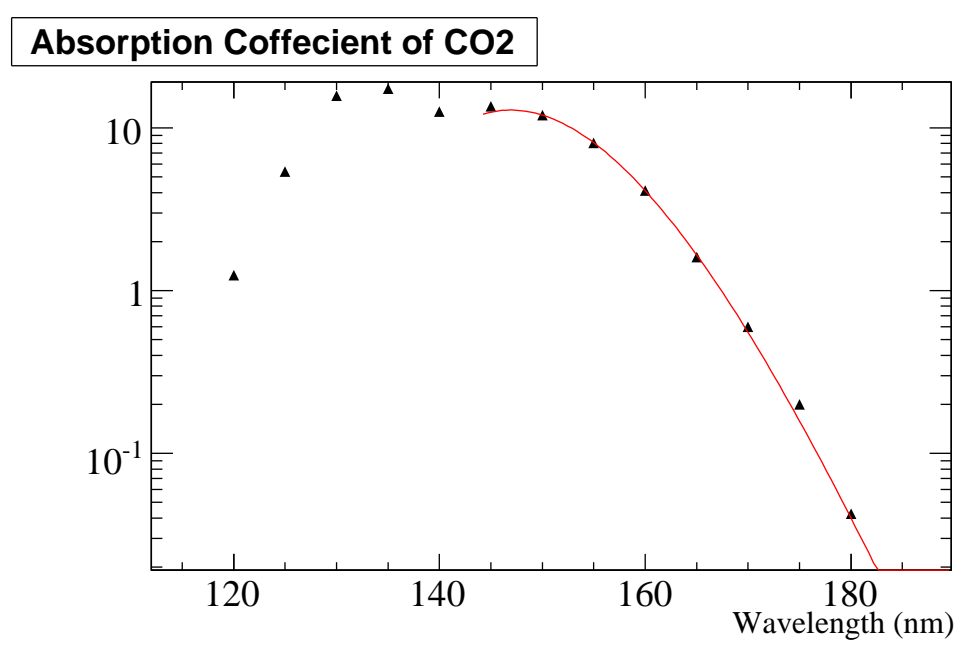

Figure 10.2: Absorption coefficient of carbon dioxide as a function of wavelength with overlaid parameterization.

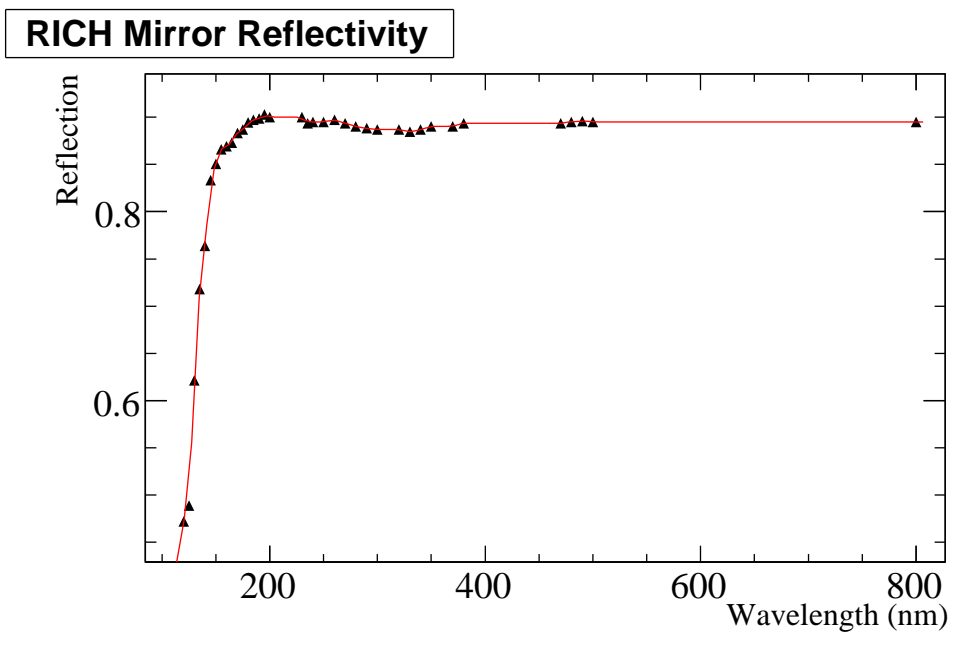

Figure 10.3: Reflectance of RICH mirrors as a function of wavelength. Data courtesy of SELEX collaboration [10]. 


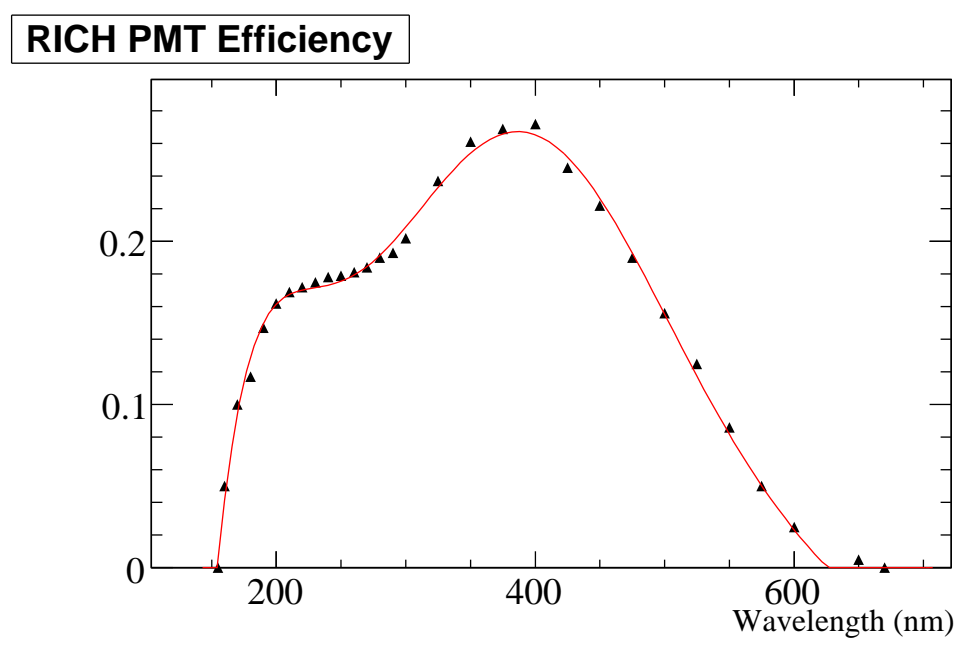

Figure 10.4: RICH Hamamatsu photomultiplier tube efficiency as a function of wavelength. Data courtesy of the SELEX collaboration [10].

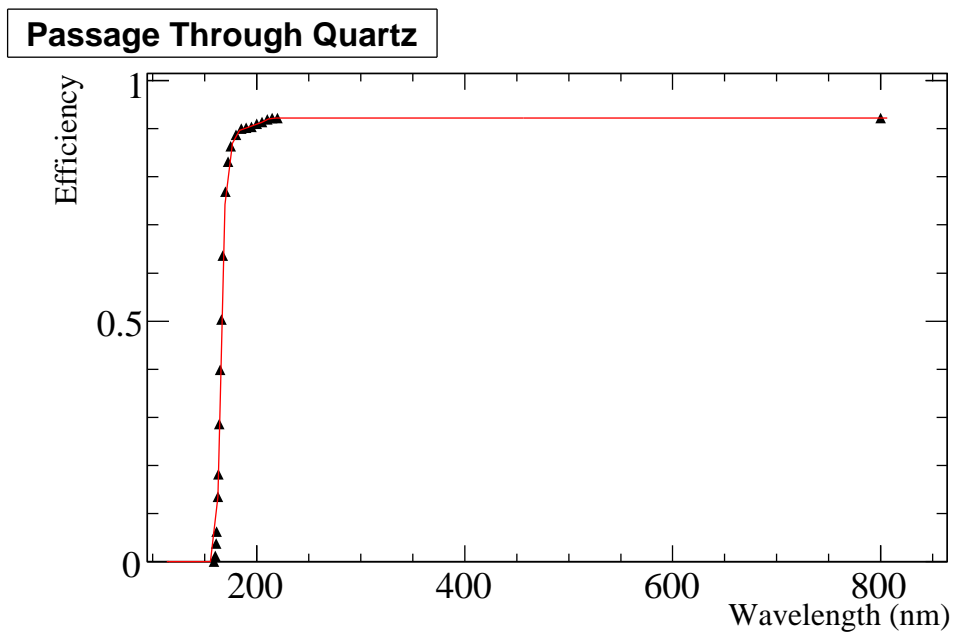

Figure 10.5: RICH quartz window transmission as a function of wavelength. Data courtesy of SELEX collaboration [10]. 


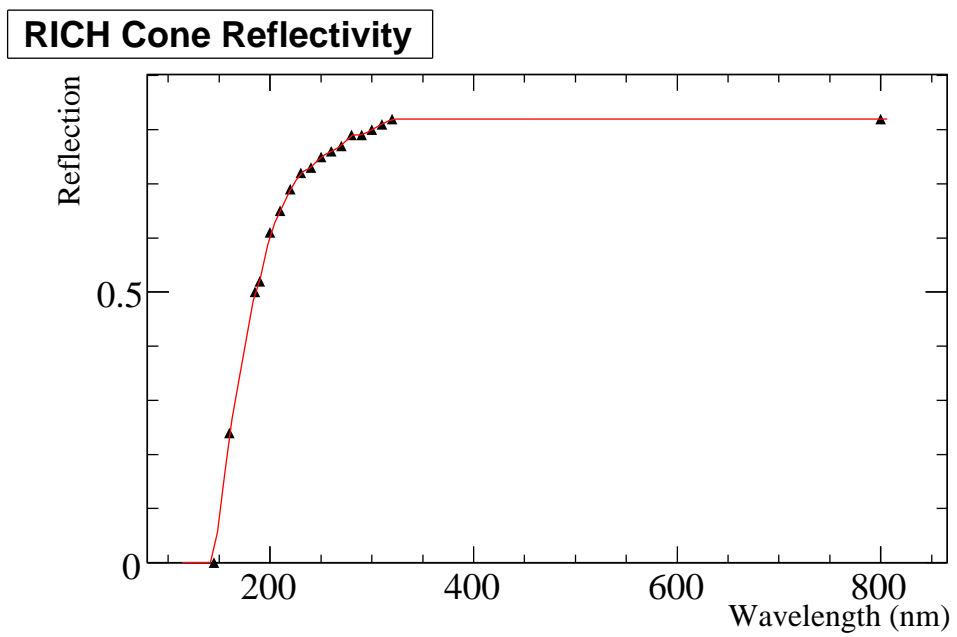

Figure 10.6: Reflectance of RICH PMT cones as a function of wavelength. Data courtesy of SELEX collaboration [10].

length is $\phi / \pi$. Which means the geometric acceptance is

$$
G_{P M T}=\frac{1}{\pi} \arccos \left(\frac{R_{c}^{2}+R_{0}^{2}+R_{P M T}^{2}}{2 R_{0} R_{c}}\right),
$$

where $R_{c}$ is the ring radius corresponding to the Cherenkov angle, $R_{0}$ is the distance between the tube center and the ring center, and $R_{P M T}$ is the radius of the PMT.

The number of photoelectrons seen by tube $i$ is then

$$
N_{p e}^{i}=\int_{0}^{L} \int_{\lambda_{1}}^{\lambda_{2}} \frac{2 \pi \alpha}{\lambda^{2}}\left(1-\frac{1}{n^{2}(\lambda) \beta^{2}}\right) \exp \left(-\mu(\lambda)\left(F_{L}+x\right)\right) E_{f}(\lambda) G_{P M T}^{i} \partial \lambda \partial x .
$$

where $\mu(\lambda)$ is the absorption coefficient of $\mathrm{CO}_{2}$ (Figure 10.2), $L$ is the path 


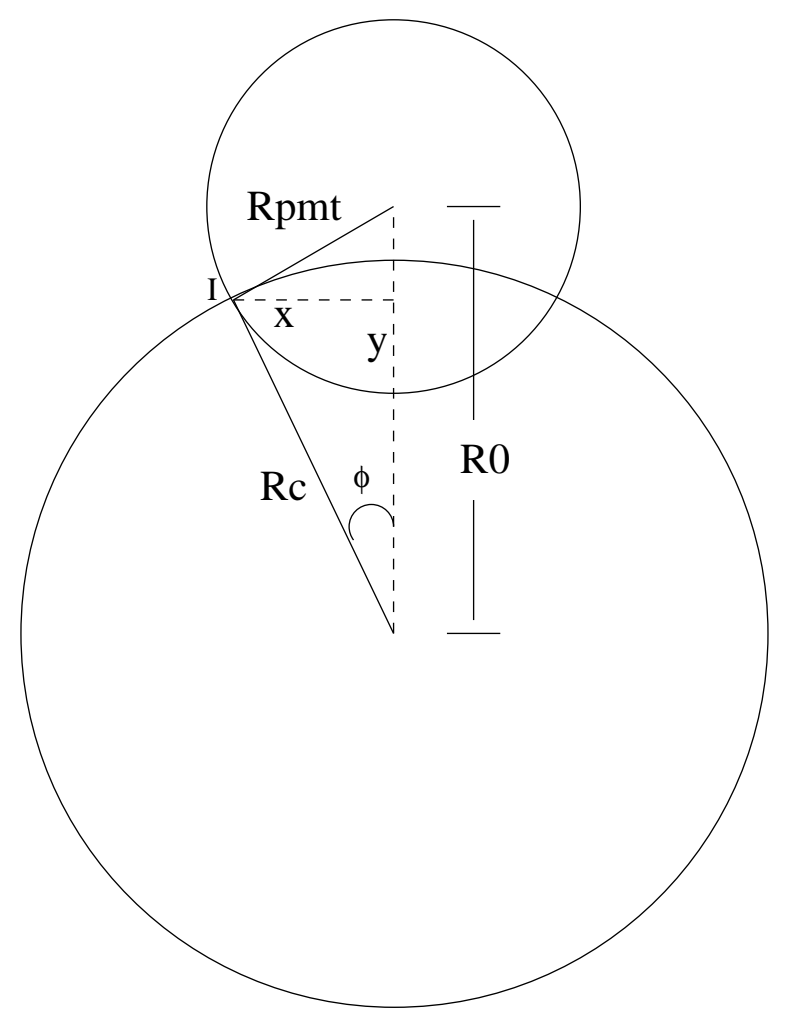

Figure 10.7: Phototube geometric acceptance. 


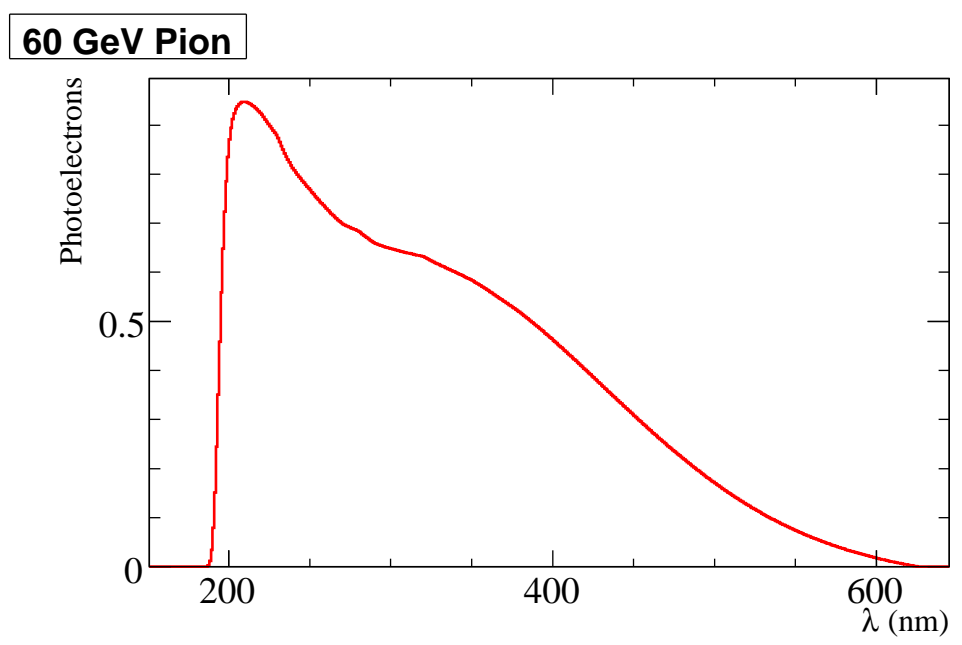

Figure 10.8: Npe vs. wavelength for $60 \mathrm{GeV}$ pion. Absorption and all efficiency factors are included.

length of the particle through the radiator volume, $F_{L}$ is the mirror focal length, $\lambda_{1}$ and $\lambda_{2}$ represent the PMT sensitivity range, and $E_{f}(\lambda)$ is the product of all efficiency factors: mirror (Figure 10.3) and cone (Figure 10.6) reflectance, quartz window transmission (Figure 10.5), and PMT quantum and collection efficiencies (Figure 10.4). Note that this expression is for the case of no scattering. To include scattering, it must be transformed into an effective integral over angle. The number of photoelectrons vs. wavelength for a $60 \mathrm{GeV} / c$ pion is shown in Figure 10.8. This includes all efficiency factors and absorption. 


\subsubsection{Light Smearing}

Smearing of the light distribution is modeled as a Gaussian with three contributions to the width. The first is a constant value, which we label $\sigma_{0}$. It is due to imperfect focusing by the mirrors and finite resolution of track direction. Thus it is an intrinsic characteristic of the detector itself.

Since $\mathrm{CO}_{2}$ is a dispersive medium, variations in the refractive index over the length of the radiator volume contribute to the Cherenkov angle resolution. From Equation 5.1, this leads to a term proportional to $1 / \tan \theta_{c}$ [19]. This is accounted for by incorporating this term into the smearing width.

Multiple Coulomb scattering of the charged track as it traverses the RICH volume smears the light distribution. It is sufficiently well described by a Gaussian for small deflection angles. From [13] the width of the angular distribution is given by:

$$
\sigma_{m s}=\frac{13.6 \mathrm{MeV}}{\beta p} z \sqrt{x / X_{0}}\left[1+0.038 \ln \left(x / X_{0}\right)\right]
$$

where $x / X_{0}$ is the thickness of the scattering medium in radiation lengths. The radiation length of $\mathrm{CO}_{2}$ is $18310 \mathrm{~cm}$ [13] and with a path through the $\mathrm{RICH}$ of $1023 \mathrm{~cm}$ for straight going beam particles, the final expression for multiple scattering is

$$
\sigma_{m s}=\frac{0.00286 \mathrm{GeV}}{\beta p}
$$

Multiple scattering contributes about $0.05 \mathrm{mrad}$ to the smearing width. This 
is an order of magnitude lower than $\sigma_{0}$ and the dispersive term which are each approximately $0.4 \mathrm{mrad}$.

Combining all three widths together gives the total light smearing width:

$$
\sigma\left(\theta_{c}\right)=\sqrt{\sigma_{0}^{2}+\frac{\sigma_{n}^{2}}{\tan ^{2} \theta_{c}}+\sigma_{m s}^{2}}
$$

Using this result, the amount of light seen at angle $\theta$ from Cherenkov angle $\theta_{c}$ due to smearing can be calculated. Applying to Eqn. 10.2 transforms it into an effective integral over angle:

$$
\begin{array}{r}
N_{p e}^{i}=\int_{0}^{L} \int_{\theta_{1}}^{\theta_{2}} \frac{2 \pi \alpha}{\lambda^{2}}\left(1-\frac{1}{n^{2}(\lambda) \beta^{2}}\right) \exp \left(-\mu(\lambda)\left(F_{L}+x\right)\right) E_{f}(\lambda) G_{P M T}^{i} \\
\otimes S\left(\theta, \theta_{c}\right) \partial \theta \partial x .(10.6)
\end{array}
$$

with the new factor $S\left(\theta, \theta_{c}\right)$ representing the smearing function. Figure 10.9 shows the number of photoelectrons vs. wavelength for the $60 \mathrm{GeV} / c$ pion in Figure 10.8 after scattering is applied.

\subsubsection{Probability of PMT Hit}

With this photoelectron prediction in hand, the probability that a tube fires is given by Poisson statistics [29]:

$$
P=1-\exp \left(-N_{p e}\right)
$$




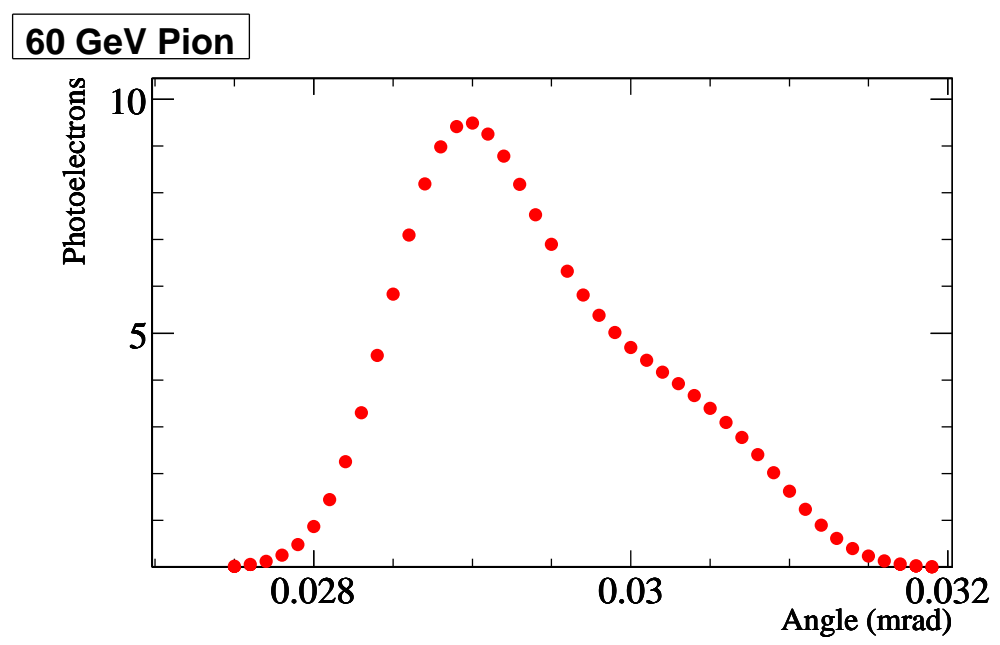

Figure 10.9: Npe vs. angle in mrad for $60 \mathrm{GeV}$ pion. Absorption and all efficiency factors are included.

which can then be used to predicted the pattern of hit tubes seen in the data. The representation of a ring presented here is more complicated than in Section 6.1.1, but the concept remains the same. The angular distribution of light produced by different particle species of the same momentum are correlated via the momentum and the index of refraction of the radiator. So again, a mono-energetic beam of pions, kaons and protons is needed and the precisely known proton and pion masses are the key to understanding systematics. 


\subsection{Data Occupancy}

Data is analyzed by accumulating occupancy distributions as a function of angle for each data set. Occupancy for a given PMT is defined as the fraction of events considered in which the tube fired. It is therefore a measure of the probability that tube will fire. This is averaged for all tubes which fall within each Cherenkov angle bin. The angle is calculated using the position of the tube center relative to the ring center prediction from track segments and the focal length of the mirror. Bin size is $0.2 \mathrm{mrad}$. This is fine enough to resolve features of the distribution while not so fine as to leave gaps due to segmentation of the detector.

Occupancy distributions are made separately for $\pi / K / p$ events. Hamamatsu R760 and FEU60 tubes are considered separately, as are tubes associated with mirrors 8 and 9 . Bad channels and hot columns are ignored. The fraction of events in which is tube as flagged as bad is recorded for use in the prediction.

Selecting good tubes is crucial. Figure 10.10 shows a map of the accumulated hits for $40 \mathrm{GeV} / c$ proton events. Each box represents a PMT with the size proportional to the content. Mirror 8 tubes are on the right half of the ring, mirror 9 tubes on the left. There is a region between columns 45 and 53 where the number of hits is much lower than other portions of the ring. This likely due to hot columns, and should have accounted for by the recorded bad fractions. However, the bad fractions for these tubes are not 
low enough to account for such a large discrepancy in the number of hits. There is a similar region between columns 37 and 45 , though the difference is not as pronounced. A similar pattern is seen in all data sets. Also, columns 31 and 60 were found to be consistently low in all data sets. Occupancy plots were redone with these columns ignored.

This had a significant impact on the shape of some distributions, particularly for protons. Proton rings have fewer hits than pion and kaon rings, and hot columns often appeared near proton ring edges.

In the $37 \mathrm{GeV}$ mirror 9 data set (Figure 10.20), the FEU60 pion peak shifted down by just under 1\%. The proton Hamamatsu peak was cut off below $13 \mathrm{mrad}$, and the peak shifted downward by $3 \%$. The kaon Hamamatsu peak shifted down by 1\%. For the mirror 8 data set (Figure 10.21) proton distributions were cut off below $17 \mathrm{mrad}$ for Hamamatsu tubes and $14 \mathrm{mrad}$ for FEU60 tubes. The kaon FEU60 shifted down by $1 \%$ and became much better defined, with the root mean square (RMS) decreasing by $3.6 \%$.

In the $40 \mathrm{GeV}$ mirror 9 data set (Figure 10.22), the proton FEU60 distribution had a more pronounced shoulder, decreasing from $60 \%$ of the peak to $40 \%$ of the peak. For the mirror 8 set (Figure 10.23), the pion Hamamatsu peak shifted down by just under $1 \%$. The double peaked structure in the proton Hamamatsu distribution was more pronounced, with the peak on the right $17 \%$ higher than the left.

In the $42 \mathrm{GeV}$ mirror 9 data set (Figure 10.24), the shoulder in the proton Hamamatsu distribution decreased from $60 \%$ of the peak to $40 \%$, while the 


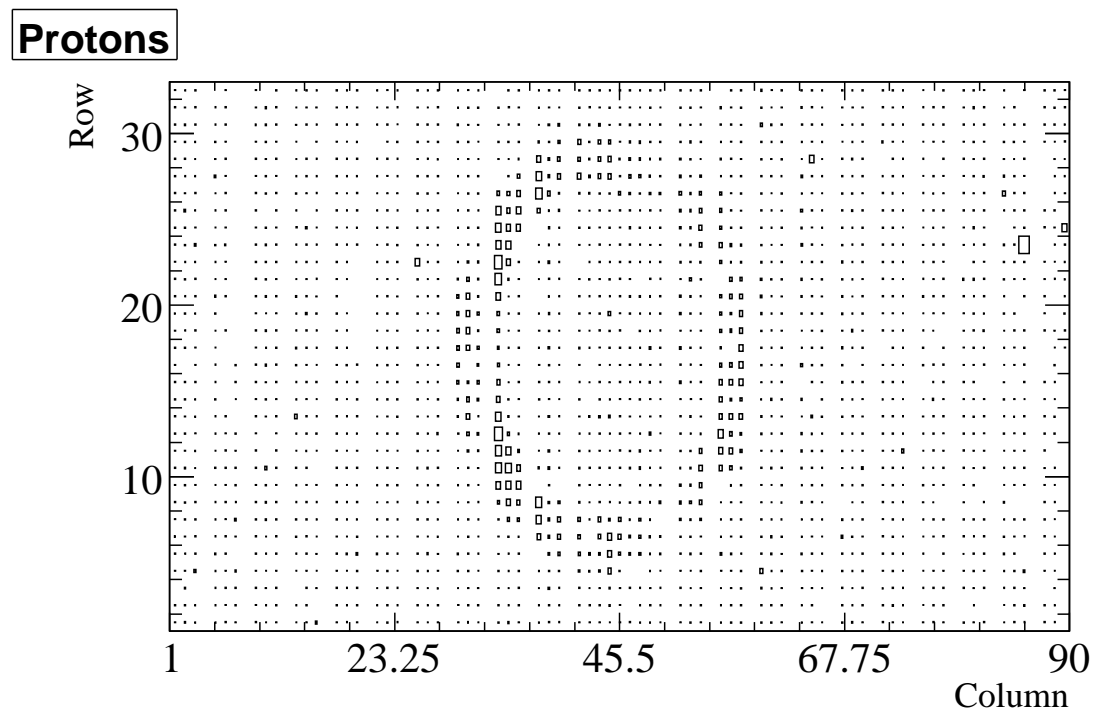

Figure 10.10: Accumulated hit map for $40 \mathrm{GeV}$ protons. Each box represents a PMT with the size proportional to the number of hits seen by that PMT.

shoulder in the FEU60 distribution was not present. The peak also shifted down by 5\%. For the mirror 8 set (Figure 10.25), the proton Hamamatsu peak shifted down by $3 \%$.

Higher momentum data sets are shown in Figures 10.26 - 10.33. Changes in these sets were less significant, with some peaks shifting down by $1 \%$ or less. These shifts are due to changes in shape in the region near the peak and not shifts in the entire distribution. In general, peak occupancy increased by nearly $40 \%$ for protons, $10 \%$ for kaons and $5 \%$ for pions. 


\subsection{Predicting Occupancy}

The RICH reconstruction software handling light prediction is implemented as a $\mathrm{C}++$ class. Upon creation of the class object, several parameters that are functions of wavelength are sampled in steps of $1 \mathrm{~nm}$ over the range of PMT sensitivity, from $169 \mathrm{~nm}$ to $625 \mathrm{~nm}$. These values are then stored in arrays. The parameters sampled are:

1. Product of all efficiencies

2. Refractive index of $\mathrm{CO}_{2}$ at STP

3. Refractive index of air at STP

4. Absorption coefficient of $\mathrm{CO}_{2}$

Also upon construction normalized Gaussian values are tabulated in steps of $0.01 \sigma$. This is needed to speed up smearing of the light distribution from scattering. The index of refraction of air is calculated using a parameterization from the CRC [2] at $1 \mathrm{~atm}, 20^{\circ} \mathrm{C}$ and scaled to STP.

Once the object has been created, several parameters need to be set:

1. Position of ring center

2. Path length through $\mathrm{CO}_{2}$

3. Particle mass

4. Particle momentum 
5. Density of $\mathrm{CO}_{2}$ gas

6. Scattering parameters

7. Level of air contamination

Other parameters can then be calculated: $\beta$ of the particle, the final index refraction combining $\mathrm{CO}_{2}$ and air and adjusted for density, the Cherenkov angle and its tangent (equal to ring radius divided by focal length) from Eqn. 5.1 , light smearing width, and the number of photons per $\mathrm{nm}$ of wavelength by integrating Eqn. 10.2 over path length. All except $\beta$ are functions of wavelength and are thus tabulated in steps of $1 \mathrm{~nm}$ just as the initial parameters. Note that the geometric acceptance factor is inside the wavelength integral. It is applied when this integral is evaluated over each tube individually.

Refractive indices of $\mathrm{CO}_{2}$ and air are combined using the empirical law of Avogadro-Biot-Beer-Landolt-Christiansen-Wintgen [21]:

$$
n_{t}=\psi_{m} n_{m}+\psi_{p} n_{p}
$$

where $\psi_{m}$ and $\psi_{p}$ are the volume fractions of the medium of dispersion and the dispersed substances respectively. The combined index is scaled from STP using the Lorenz-Lorentz law [39]:

$$
\frac{n^{2}-1}{n^{2}+2} \frac{1}{\rho}=\text { const. }
$$


Light smearing is implemented via a mixing matrix between $\theta$ and $\theta_{c}$., with $\theta$ sampled in steps of $0.01 \mathrm{mrad}$ from 0 to $35 \mathrm{mrad}$. Note that there is a 1:1 relationship between $\theta_{c}$ and $\lambda$ defined by the values already tabulated. Simply applying the mixing matrix to the photoelectron table converts it from a function of $\theta_{c}$ to $\theta$.

We now have table of values that can be used to compute the integral in Eqn. 10.6. This is done for a particular PMT by looping over the table and adding up the entries while applying the geometric acceptance given by Eqn. 10.1 .

Data distributions are accumulated over a period of several days, during which conditions such as gas density, momentum, and ring center vary. The prediction must also reflect this. It is integrated over density and ring horizontal and vertical center position ranges from the data weighted based on the distributions for mirror 9. Mirror 8 ring center is derived from mirror 9 using the linear fit results in Figure 9.12. Values are sampled at 11 evenly spaced points about the peak. An integral over momentum is also included. The shape is assumed to be Gaussian and is sampled at 7 points between $\pm 3 \sigma$. The light distribution is thus calculated about 9300 times to make one prediction. Increasing the number of sampled points did not significantly affect the prediction.

The number of photoelectrons is predicted for each tube based on the current parameters. They are multiplied by the predicted fraction that hits the mirror array as described in Section 9.2. This is more important for 
mirror 8 because of the cutout section. The probability of firing is calculated using Eqn. 10.7 with the noise rates from Section 8.2.4 included. The tubes are binned in angle and averaged just like the data. Individual tubes are weighted down according the fraction of events in which they were flagged as bad. Columns that were ignored when accumulating data were also ignored in the predictions. The final accumulated distribution serves as a prediction of the PMT occupation.

Differences in $\pi / K / p$ production spectra off the primary target means that average beam momentum may not be the same for all three. To account for this, a FLUKA $[18,17]$ simulation of the MIPP primary copper target was ran with 30 million $120 \mathrm{GeV} / c$ protons on target (POT). Momentum distributions were fit piecewise to an exponential function over ranges of \pm 5 $\mathrm{GeV} / c$ about central values of $40 \mathrm{GeV} / c, 50 \mathrm{GeV} / c$ and $60 \mathrm{GeV} / c$.

The momentum bite of the MIPP secondary beamline is on the order of $0.5 \%$. The less than $1 \%$ variation in the current of the momentum selecting magnet previously shown in Figure 8.1 effectively increases the bite. Fit results from FLUKA were integrated over a Gaussian with $\sigma$ equal to $0.5 \%$ of the mean, with a range of $\pm 3 \sigma$. This was done in steps of $1 \mathrm{GeV} / c$ from 35 $\mathrm{GeV} / c$ to $65 \mathrm{GeV} / c$ for the mean value. Proton and kaon relative deviations from pion momentum are plotted vs. pion momentum in Figure 10.14. The results fit very well to a straight line. Deviations are on the order of 10 ppm for protons and $1 \mathrm{ppm}$ for kaons, much less than the $100 \mathrm{ppm}$ goal for this measurement. The effect of changing the momentum width was studied. 


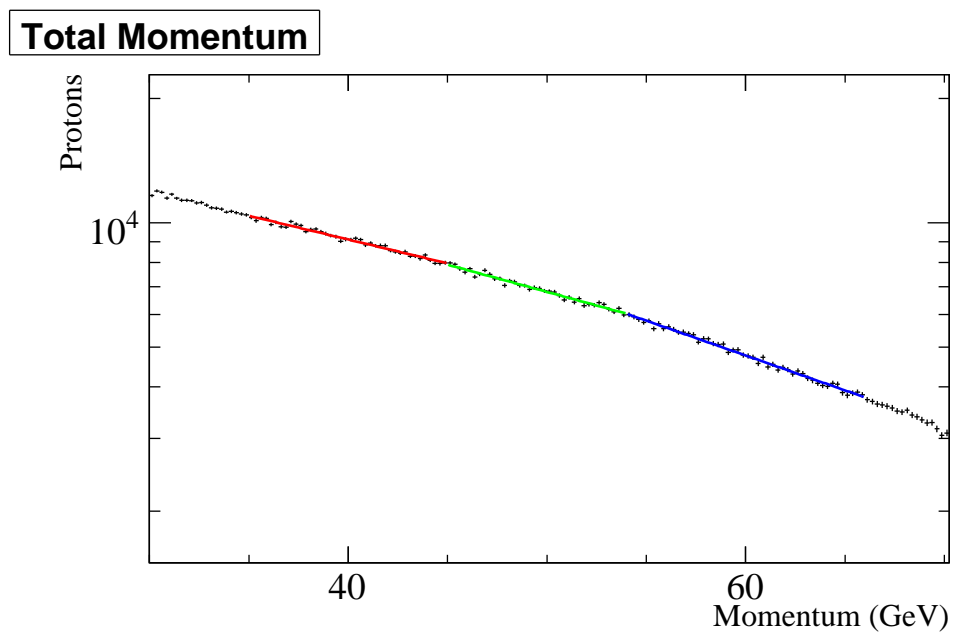

Figure 10.11: Proton momentum spectrum from FLUKA simulation of $\mathrm{Cu}$ target. A piecewise exponential fit is superimposed.

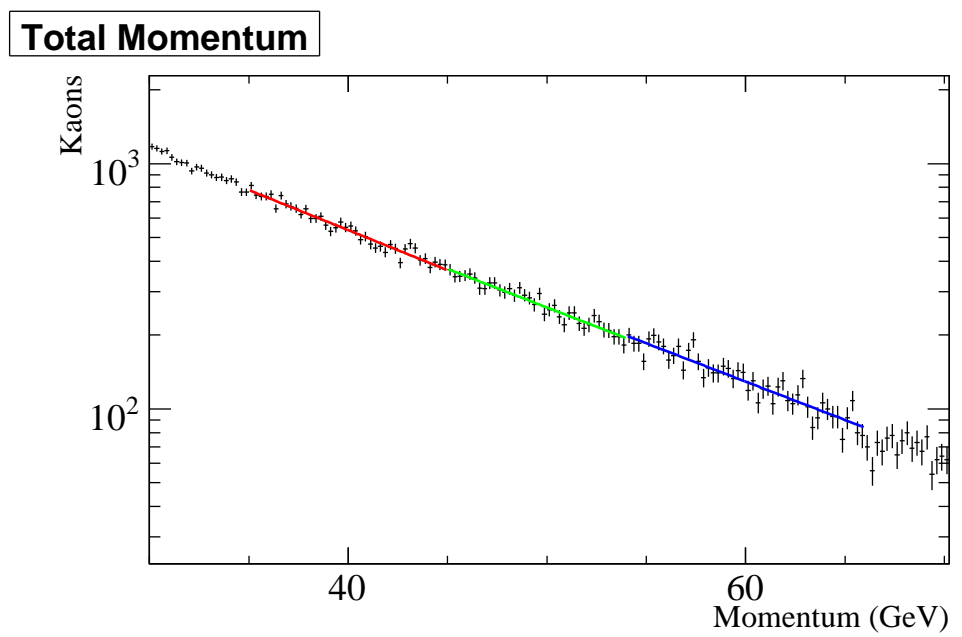

Figure 10.12: Kaon momentum spectrum from FLUKA simulation of $\mathrm{Cu}$ target. A piecewise exponential fit is superimposed. 


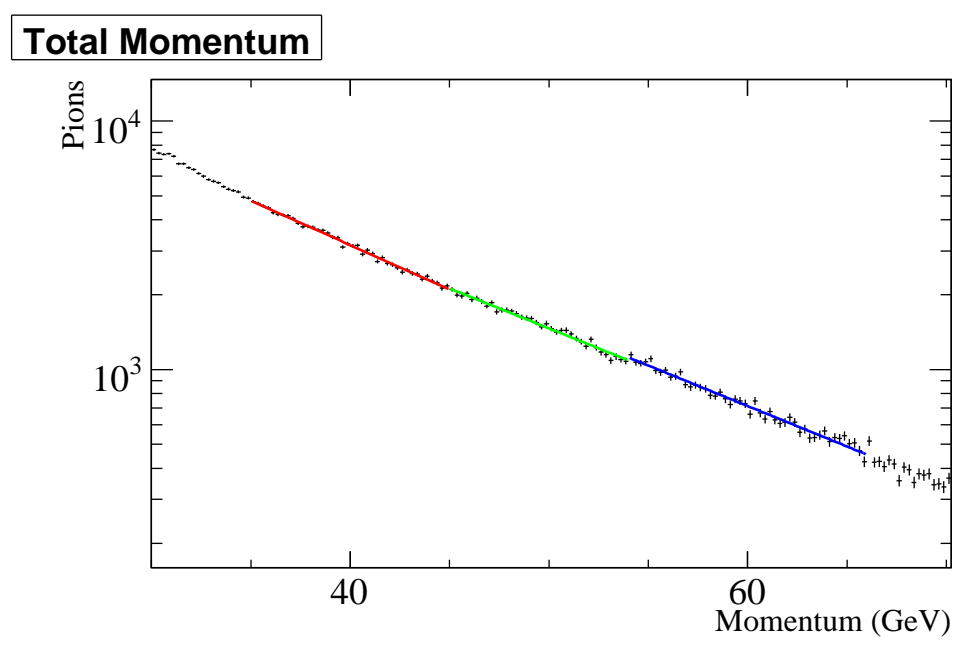

Figure 10.13: Pion momentum spectrum from FLUKA simulation of $\mathrm{Cu}$ target. A piecewise exponential fit is superimposed.

Changes were not numerically significant until the width was increased by an order of magnitude.

Only the pion momentum is a parameter in the prediction. The proton and kaon momenta are derived from the FLUKA results.

\subsection{Fitting Procedure}

Predictions have been made assuming R760 efficiency. This is not the case for FEU60 tubes, which is why the two types are considered separately. The overall efficiency scale may also by off systematically. Normalization enters into the number of photoelectrons and not the probability, so both predictions and data are converted to photoelectrons by solving Eqn. 10.7. The area 


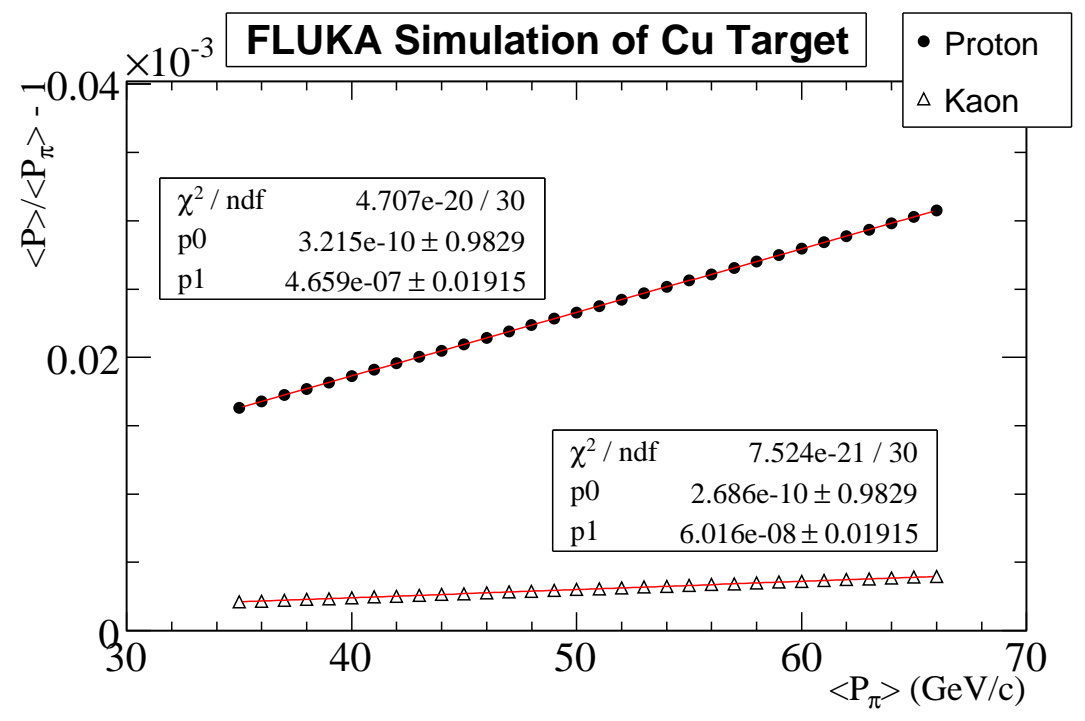

Figure 10.14: Proton and kaon relative deviations from pion momentum vs pion momentum. Results from FLUKA simulation of MIPP primary copper target with 30 million $120 \mathrm{GeV} / c$ POT. 


\begin{tabular}{l|l|l}
\hline \hline Parameter Name & Description & Seed Value \\
\hline$\sigma_{0}$ & Intrinsic smearing width & $2.0 \times 10^{-4} \mathrm{rad}$ \\
$\sigma_{N}$ & Dispersive smearing width & $9.0 \times 10^{-6} \mathrm{rad}$ \\
DensSf & Density ratio scaling factor & 0.97 \\
DensOS & Density ratio offset & 0.008 \\
AirFrac & Level of air contamination in $\mathrm{CO}_{2}$ & $100 \mathrm{ppm}$ \\
$P_{\pi}$ & Central pion momentum & 0.98 of nominal \\
$\sigma_{P}$ & Gaussian momentum width & $0.5 \%$ \\
$m_{K}$ & Charged kaon mass & $493.677 \mathrm{MeV}$ \\
\hline \hline
\end{tabular}

Table 10.1: List of fit parameters and initial seed values.

of the prediction is scaled to match the data, and then the prediction is converted back to a probability or occupancy.

A $\chi^{2}$ is then calculated between the data and prediction using bins near the peaks. The values for $\pi / K / p$ for both R760 and FEU60 tubes are summed to get the final $\chi^{2}$. Minimization is handled by Minuit by varying the smearing parameters $\sigma_{0}$ and $\sigma_{n}$, a scaling factor and an offset for the density, the level of air contamination, the central pion momentum and momentum width. The proton, pion and kaon masses are held fixed at their PDG [13] values. Reasonable initial values were found by hand, with scattering width on the order of $10^{-4}$, density scaling of 0.97 , momentum width of $0.5 \%$ and central momenta 0.98 times nominal. Table 10.1 lists all the parameters with initial seed values. The level of air contamination in the RICH was known to be less than 1000 ppm, so it was constrained in the fit to not exceed this value.

After 200 iterations, the kaon mass is allowed to vary along with all other 


\begin{tabular}{l|ccc}
\hline \hline Data Set & $\chi^{2}$ & NDF & Scale Factor \\
\hline $37 \mathrm{GeV}$ Mirror 8 & 751280 & 135 & 74.6 \\
$37 \mathrm{GeV}$ Mirror 9 & 804659 & 154 & 72.3 \\
$40 \mathrm{GeV}$ Mirror 8 & $1.36259 \times 10^{6}$ & 144 & 97.3 \\
$40 \mathrm{GeV}$ Mirror 9 & $1.39644 \times 10^{6}$ & 144 & 98.5 \\
$42 \mathrm{GeV}$ Mirror 8 & 737243 & 156 & 68.7 \\
$42 \mathrm{GeV}$ Mirror 9 & 557398 & 156 & 59.8 \\
$56 \mathrm{GeV}$ Mirror 8 & 189219 & 144 & 36.2 \\
$56 \mathrm{GeV}$ Mirror 9 & 185257 & 144 & 35.9 \\
$60 \mathrm{GeV}$ Field Off Mirror 8 & 923247 & 146 & 79.5 \\
$60 \mathrm{GeV}$ Field Off Mirror 9 & $1.20764 \times 10^{6}$ & 146 & 90.9 \\
$60 \mathrm{GeV}$ Field On Mirror 8 & 496364 & 144 & 58.7 \\
$60 \mathrm{GeV}$ Field On Mirror 9 & 575235 & 144 & 63.2 \\
$63 \mathrm{GeV}$ Mirror 8 & 102808 & 144 & 26.7 \\
$63 \mathrm{GeV}$ Mirror 9 & 863250 & 144 & 77.4 \\
\hline \hline
\end{tabular}

Table 10.2: $\chi^{2}$ and number of degrees of freedom for each data set. Errors bars were scaled by $\sqrt{\chi^{2} / N D F}$ and the error matrix calculated.

parameters and the minimization continued for another 1000 iterations or until convergence. Error bars are scaled by the values in Table 10.2 so that $\chi^{2}$ per degree of freedom at the minimum is equal to 1 and the error matrix is calculated. Mirrors 8 and 9 are fit separately.

\subsection{Fit Results}

Pion distributions are very consistent between different mirrors and PMT types. Peak locations differ by less $1 \%$ with RMS widths differing by at most 5\% (Table 10.3). Data distributions are well matched by predictions, with peak locations agreeing to better than $1 \%$ and RMS widths differing by at 


\begin{tabular}{|l|cc|cc|cc|cc|}
\hline \hline \multirow{2}{*}{ Set/Mirror } & \multicolumn{4}{|c|}{ R760 } & \multicolumn{4}{c|}{ FEU60 } \\
\cline { 2 - 9 } & \multicolumn{2}{|c|}{$\langle\theta\rangle$} & \multicolumn{2}{c|}{$\theta_{R M S}$} & \multicolumn{2}{c|}{$\langle\theta\rangle$} & \multicolumn{2}{c|}{$\theta_{R M S}$} \\
& Data & Pred & Data & Pred & Data & Pred & Data & Pred \\
\hline $37 \mathrm{GeV} / 8$ & 28.9 & 29.1 & 1.8 & 2.0 & 28.9 & 29.1 & 1.9 & 2.0 \\
$37 \mathrm{GeV} / 9$ & 29.1 & 29.1 & 2.2 & 2.0 & 29.1 & 29.1 & 1.8 & 2.0 \\
\hline $40 \mathrm{GeV} / 8$ & 29.1 & 29.1 & 1.8 & 2.0 & 29.1 & 29.3 & 1.9 & 2.0 \\
$40 \mathrm{GeV} / 9$ & 29.3 & 29.5 & 2.2 & 2.0 & 29.3 & 29.5 & 1.8 & 2.0 \\
\hline $42 \mathrm{GeV} / 8$ & 28.7 & 29.3 & 1.8 & 2.0 & 28.7 & 29.3 & 2.0 & 2.0 \\
$42 \mathrm{GeV} / 9$ & 29.1 & 29.1 & 2.2 & 2.0 & 29.1 & 29.1 & 1.8 & 2.0 \\
\hline $56 \mathrm{GeV} / 8$ & 29.1 & 29.3 & 1.8 & 2.0 & 29.1 & 29.3 & 1.9 & 2.0 \\
$56 \mathrm{GeV} / 9$ & 29.3 & 29.5 & 2.2 & 2.0 & 29.5 & 29.5 & 1.8 & 2.0 \\
\hline $60 \mathrm{GeV}$ & 29.3 & 29.3 & 1.8 & 2.0 & 29.1 & 29.3 & 1.8 & 2.0 \\
Off/8 & & & & & & & & \\
$60 \mathrm{GeV}$ & 29.3 & 29.3 & 2.2 & 2.0 & 29.3 & 29.3 & 1.8 & 2.0 \\
Off/9 & & & & & & & & \\
\hline $60 \mathrm{GeV}$ & 29.3 & 29.5 & 1.8 & 2.0 & 29.3 & 29.5 & 1.8 & 2.0 \\
On/8 & & & & & & & & \\
$60 \mathrm{GeV}$ & 29.3 & 29.3 & 2.2 & 2.0 & 29.3 & 29.3 & 1.8 & 2.0 \\
On/9 & & & & & & & & \\
\hline $63 \mathrm{GeV} / 8$ & 29.3 & 29.5 & 1.8 & 2.0 & 29.3 & 29.5 & 1.8 & 2.0 \\
$63 \mathrm{GeV} / 9$ & 29.1 & 29.3 & 2.2 & 2.0 & 29.3 & 29.3 & 1.8 & 2.0 \\
\hline \hline
\end{tabular}

Table 10.3: Average and RMS angles for pion occupation distributions. Values from data and predictions are compared. Numbers are in mrad.

most 10\%. The edges of the peak are also well fit. The exception is the 42 GeV mirror 8 data set in Figure 10.25, where the peak locations are off by $2 \%$ and the edges do not match well. The fit for this set is generally poor and the result is not included in the final analysis.

Pions are affected least by bad channel regions in the array since they do not overlap much with them. They also have a high number of hits, typically 25 to 30 . 
Proton distributions for the low momentum data sets show many features that are not well predicted, especially the mirror 8 distributions. Many of them have shoulders on the high angle edge and the $40 \mathrm{GeV}$ mirror 8 distributions (Figure 10.23) are double peaked. For $37 \mathrm{GeV}$, much of the data was cut out by the good column restriction.

As a result, the peak locations and RMS widths for mirrors 8 and 9 , listed in Table 10.4, are not always consistent. Differences are about 4-15\% for low momentum data sets. Hamamatsu and FEU distributions for the same mirror show similar differences. Higher momentum data sets are more consistent, differing by no more than $1.5 \%$. Despite this, the edges of the peaks are reasonably well fit. This is more important for determining the location of predictions peaks than matching structure within the peak.

These discrepancies in the prediction peaks are likely due to regions of bad channels in the PMT array, which overlap significantly with proton rings. Protons also have fewer hits to start with, usually around 15 to 20.

Like pions, kaons are reasonably consistent between mirrors and PMT types. Peak locations agree to within $1 \%$ and RMS widths differ by at most $15 \%$ (Table 10.5). Prediction peaks also generally match within 1\%. An exception is the $40 \mathrm{GeV}$ mirror 8 set in Figure 10.23. There is a slanted structure in the peak which is not predicted, although the edges are well fit. The prediction is a bit low near the peak center for the higher momentum mirror 8 sets. Again, the edges are well fit.

Table 10.6 lists the fit results for each parameter except the kaon mass 


\begin{tabular}{|l|cc|cc|cc|cc|}
\hline \hline \multirow{2}{*}{ Set/Mirror } & \multicolumn{4}{|c|}{ R760 } & \multicolumn{4}{c|}{ FEU60 } \\
\cline { 2 - 9 } & \multicolumn{2}{|c|}{$\langle\theta\rangle$} & \multicolumn{2}{c|}{$\theta_{R M S}$} & \multicolumn{2}{c|}{$\langle\theta\rangle$} & \multicolumn{2}{c|}{$\theta_{R M S}$} \\
& Data & Pred & Data & Pred & Data & Pred & Data & Pred \\
\hline $37 \mathrm{GeV} / 8$ & 16.7 & 16.9 & 1.1 & 3.5 & 14.5 & 14.9 & 1.9 & 3.5 \\
$37 \mathrm{GeV} / 9$ & 14.1 & 14.7 & 2.5 & 3.5 & 14.5 & 14.7 & 3.1 & 3.5 \\
\hline $40 \mathrm{GeV} / 8$ & 18.5 & 17.9 & 2.4 & 3.1 & 18.5 & 17.7 & 2.4 & 3.1 \\
$40 \mathrm{GeV} / 9$ & 17.3 & 17.5 & 2.9 & 3.1 & 17.5 & 17.5 & 3.3 & 3.1 \\
\hline $42 \mathrm{GeV} / 8$ & 17.9 & 18.5 & 2.4 & 3.0 & 18.7 & 18.7 & 2.8 & 3.0 \\
$42 \mathrm{GeV} / 9$ & 18.7 & 18.7 & 3.0 & 3.0 & 18.5 & 18.7 & 2.9 & 3.0 \\
\hline $56 \mathrm{GeV} / 8$ & 23.5 & 24.1 & 2.0 & 2.2 & 23.9 & 23.9 & 2.4 & 2.2 \\
$56 \mathrm{GeV} / 9$ & 23.9 & 23.9 & 2.3 & 2.2 & 23.7 & 24.1 & 2.0 & 2.2 \\
\hline $60 \mathrm{GeV}$ & 24.3 & 24.5 & 2.1 & 2.4 & 24.5 & 24.1 & 2.5 & 2.4 \\
Off/8 & & & & & & & & \\
$60 \mathrm{GeV}$ & 24.5 & 24.7 & 2.4 & 2.4 & 24.5 & 24.7 & 2.1 & 2.4 \\
Off/9 & & & & & & & & \\
\hline $60 \mathrm{GeV}$ & 24.7 & 24.7 & 2.1 & 2.4 & 24.7 & 24.9 & 2.6 & 2.4 \\
On/8 & & & & & & & & \\
$60 \mathrm{GeV}$ & 24.7 & 24.5 & 2.4 & 2.4 & 24.5 & 24.7 & 2.1 & 2.4 \\
On/9 & & & & & & & & \\
\hline $63 \mathrm{GeV} / 8$ & 25.1 & 25.1 & 2.0 & 2.3 & 25.1 & 25.1 & 2.4 & 2.3 \\
$63 \mathrm{GeV} / 9$ & 25.1 & 25.1 & 2.1 & 2.3 & 24.7 & 24.9 & 2.1 & 2.3 \\
\hline
\end{tabular}

Table 10.4: Average and RMS angles for proton occupation distributions. Values from data and predictions are compared. Numbers are in mrad. 


\begin{tabular}{|l|cc|cc|cc|cc|}
\hline \hline \multirow{2}{*}{ Set/Mirror } & \multicolumn{4}{|c|}{ R760 } & \multicolumn{4}{c|}{ FEU60 } \\
\cline { 2 - 9 } & \multicolumn{2}{|c}{$\langle\theta\rangle$} & \multicolumn{2}{|c|}{$\theta_{R M S}$} & \multicolumn{2}{c|}{$\langle\theta\rangle$} & \multicolumn{2}{c|}{$\theta_{R M S}$} \\
& Data & Pred & Data & Pred & Data & Pred & Data & Pred \\
\hline $37 \mathrm{GeV} / 8$ & 25.9 & 26.1 & 1.9 & 1.9 & 25.9 & 26.1 & 1.9 & 1.9 \\
$37 \mathrm{GeV} / 9$ & 26.1 & 26.3 & 1.7 & 1.9 & 26.3 & 26.3 & 2.0 & 1.9 \\
\hline $40 \mathrm{GeV} / 8$ & 26.5 & 26.7 & 2.2 & 2.0 & 26.5 & 26.7 & 1.9 & 2.0 \\
$40 \mathrm{GeV} / 9$ & 26.7 & 26.9 & 1.8 & 2.0 & 26.7 & 26.9 & 2.1 & 2.0 \\
\hline $42 \mathrm{GeV} / 8$ & 26.3 & 26.7 & 2.2 & 2.0 & 26.5 & 26.7 & 1.8 & 2.0 \\
$42 \mathrm{GeV} / 9$ & 26.9 & 26.9 & 1.8 & 2.0 & 26.9 & 26.9 & 2.2 & 2.0 \\
\hline $56 \mathrm{GeV} / 8$ & 28.1 & 28.1 & 2.0 & 1.9 & 27.9 & 28.1 & 1.7 & 1.9 \\
$56 \mathrm{GeV} / 9$ & 28.3 & 28.3 & 1.8 & 1.9 & 28.3 & 28.3 & 2.0 & 1.9 \\
\hline $60 \mathrm{GeV}$ & 28.3 & 28.3 & 2.1 & 2.0 & 28.3 & 28.3 & 1.6 & 1.9 \\
Off/8 & & & & & & & & \\
$60 \mathrm{GeV}$ & 28.1 & 28.3 & 1.8 & 1.9 & 28.1 & 28.1 & 2.0 & 1.9 \\
Off/9 & & & & & & & & \\
\hline $60 \mathrm{GeV}$ & 28.3 & 28.5 & 2.1 & 1.9 & 28.1 & 28.3 & 1.6 & 1.9 \\
On/8 & & & & & & & & \\
$60 \mathrm{GeV}$ & 28.1 & 28.3 & 1.7 & 1.9 & 28.1 & 28.3 & 2.0 & 1.9 \\
On/9 & & & & & & & & \\
\hline $63 \mathrm{GeV} / 8$ & 28.3 & 28.3 & 2.0 & 2.0 & 28.1 & 28.5 & 1.7 & 2.0 \\
$63 \mathrm{GeV} / 9$ & 28.5 & 28.3 & 1.9 & 2.0 & 28.3 & 28.3 & 2.0 & 2.0 \\
\hline
\end{tabular}

Table 10.5: Average and RMS angles for kaon occupation distributions. Values from data and predictions are compared. Numbers are in mrad. 
by data set. Values for the scattering parameters are consistent for low momentum mirror 9 sets. $\sigma_{0}$ is about $3.7 \times 10^{-4}$, varying by no more than $5 \%$, while $\sigma_{N}$ is approxiamtley $9.0 \times 10^{-6}$ with a $1 \%$ variation. Low momentum mirror 8 sets are similarly consistent, although $\sigma_{0}$ is $40 \%$ larger. This is not unexpected given that mirror 8 RMS widths in Tables 10.3, 10.4, and 10.5 are higher than those of mirror 9. There are variations as large as $40 \%$ in the higher momentum sets. The mirror 8 scattering parameters consistently higher than mirror 9 for these sets as well.

Except for the $37 \mathrm{GeV}$ sets, the pion momentum result is consistently lower than nominal by $0.5 \%$ to $3 \%$, with largest deviation in the $63 \mathrm{GeV}$ sets. Differences between mirror sets of the same momentum are at most $1.5 \%$. The momentum widths for mirror 9 sets are mostly near $0.5 \%$, with the $37 \mathrm{GeV}$ and $63 \mathrm{GeV}$ sets a bit lower at $0.1 \%$ and $0.2 \%$ respectively. For mirror 8 sets the momentum width is about $1 \%$ to $2 \%$. Again, this isn't surprising since mirror 8 distributions are wider.

The density scaling factor varies by just over $1 \%$, from 0.96 to 0.97 . The offset shows a much larger variation, from -0.006 to 0.008 . These are adjustments to the density ratio, which is approximately 0.92 . The offset is less than $1 \%$ of this value and does not significantly affect the index of refraction. The refractive index is also affected by the level of air contamination, which varies greatly from $14 \mathrm{ppm}$ to $998 \mathrm{ppm}$.

The parameter correlations for $40 \mathrm{GeV}$ mirror 9 is listed in Table 10.7. The correlation between the kaon mass and air contamination level is only 


\begin{tabular}{|l|ccccccc|}
\hline \hline Set/Mirror & $\begin{array}{c}\sigma_{0} \\
(\mathrm{rad}\end{array}$ & $\begin{array}{c}\sigma_{N} \\
(\mathrm{rad})\end{array}$ & DensSF & DensOS & $\begin{array}{c}\text { AirFrac } \\
(\mathrm{ppm})\end{array}$ & $\begin{array}{c}P_{\pi} \\
(\mathrm{GeV})\end{array}$ & $\sigma_{P}$ \\
\hline $37 \mathrm{GeV} / 8$ & $5.3 \times 10^{-4}$ & $8.7 \times 10^{-6}$ & 0.961 & -0.003 & 87 & 37.3 & $1.1 \%$ \\
$37 \mathrm{GeV} / 9$ & $3.6 \times 10^{-4}$ & $9.0 \times 10^{-6}$ & 0.965 & 0.002 & 102 & 37.0 & $0.1 \%$ \\
\hline $40 \mathrm{GeV} / 8$ & $5.1 \times 10^{-4}$ & $7.7 \times 10^{-6}$ & 0.962 & -0.001 & 890 & 39.8 & $2.0 \%$ \\
$40 \mathrm{GeV} / 9$ & $3.8 \times 10^{-4}$ & $9.0 \times 10^{-6}$ & 0.970 & 0.008 & 915 & 39.3 & $0.5 \%$ \\
\hline $42 \mathrm{GeV} / 8$ & $5.1 \times 10^{-4}$ & $9.1 \times 10^{-6}$ & 0.959 & 0.006 & 897 & 41.1 & $0.7 \%$ \\
$42 \mathrm{GeV} / 9$ & $3.7 \times 10^{-4}$ & $8.9 \times 10^{-6}$ & 0.965 & -0.006 & 100 & 41.8 & $0.5 \%$ \\
\hline $56 \mathrm{GeV} / 8$ & $1.9 \times 10^{-4}$ & $1.6 \times 10^{-5}$ & 0.967 & -0.0003 & 635 & 55.1 & $0.5 \%$ \\
$56 \mathrm{GeV} / 9$ & $4.6 \times 10^{-4}$ & $9.0 \times 10^{-6}$ & 0.970 & 0.007 & 132 & 54.6 & $0.5 \%$ \\
\hline $60 \mathrm{GeV}$ & $4.0 \times 10^{-4}$ & $8.1 \times 10^{-6}$ & 0.968 & 0.007 & 998 & 58.6 & $1.7 \%$ \\
Off/8 & & & & & & & \\
$60 \mathrm{GeV}$ & $3.7 \times 10^{-4}$ & $9.2 \times 10^{-6}$ & 0.970 & 0.005 & 530 & 58.6 & $0.5 \%$ \\
Off/9 & & & & & & & \\
\hline $60 \mathrm{GeV}$ & $4.6 \times 10^{-4}$ & $8.9 \times 10^{-6}$ & 0.967 & 0.007 & 998 & 58.6 & $1.7 \%$ \\
On $/ 8$ & & & & & & & \\
$60 \mathrm{GeV}$ & $3.8 \times 10^{-4}$ & $9.0 \times 10^{-6}$ & 0.964 & 0.006 & 601 & 58.7 & $0.5 \%$ \\
On/9 & & & & & & & \\
\hline $63 \mathrm{GeV} / 8$ & $5.1 \times 10^{-4}$ & $7.7 \times 10^{-6}$ & 0.965 & 0.008 & 332 & 60.7 & $1.18 \%$ \\
$63 \mathrm{GeV} / 9$ & $3.1 \times 10^{-4}$ & $1.0 \times 10^{-5}$ & 0.957 & 0.008 & 14 & 61.4 & $0.2 \%$ \\
\hline \hline
\end{tabular}

Table 10.6: Summary of fit results for all parameters except kaon mass. 


\begin{tabular}{l|cccccccc}
\hline \hline & $\sigma_{0}$ & $\sigma_{N}$ & DensSF & DensOS & $m_{K}$ & $\sigma_{P}$ & AirFrac & $P_{\pi}$ \\
\hline$\sigma_{0}$ & 1 & -0.99 & 0.36 & -0.33 & -0.65 & 0.05 & 0.11 & -0.86 \\
$\sigma_{N}$ & -0.99 & 1 & -0.36 & 0.36 & 0.64 & -0.07 & -0.1 & 0.87 \\
DensSF & 0.36 & -0.36 & 1 & -0.93 & -0.16 & 0.02 & 0.07 & -0.35 \\
DensOS & -0.33 & 0.33 & -0.93 & 1 & 0.27 & 0.01 & -0.32 & 0.23 \\
$m_{K}$ & -0.65 & 0.64 & -0.16 & 0.27 & 1 & -0.01 & -0.07 & 0.55 \\
$\sigma_{P}$ & 0.05 & -0.07 & 0.02 & 0.01 & -0.01 & 1 & -0.01 & -0.11 \\
AirFrac & 0.11 & -0.10 & 0.07 & -0.03 & -0.07 & -0.01 & 1 & -0.09 \\
$P_{\pi}$ & -0.86 & 0.87 & -0.35 & 0.23 & 0.55 & -0.11 & -0.09 & 1 \\
\hline \hline
\end{tabular}

Table 10.7: Parameter correlations for $40 \mathrm{GeV}$ mirror 9 fit.

-0.07 . The wide variations in this parameter had very little effect on the final result. Momentum width has an even lower impact with a correlation coefficient of -0.01 . Mass is most strongly correlated with the scattering widths which affect the shape of the distribution and the momentum which affects its central value. There is a lesser though still significant correlation with the density ratio.

Kaons are close enough to $\beta=1$ at $40 \mathrm{GeV}$ and above that density has a large effect on the Cherenkov angle than does momentum. One might then expect to see a larger correlation coefficient with density than momentum. However this is not the case. Since protons are much more massive, their Cherenkov angle is more strongly affected by momentum. Proton mass is fixed in the fit, so when momentum changes the density ratio must then be changed by a much larger amount to move the proton prediction back in line with the data. This change in density affects the kaon peak more strongly than the momentum did, and so the kaon mass must be changed so the kaon 


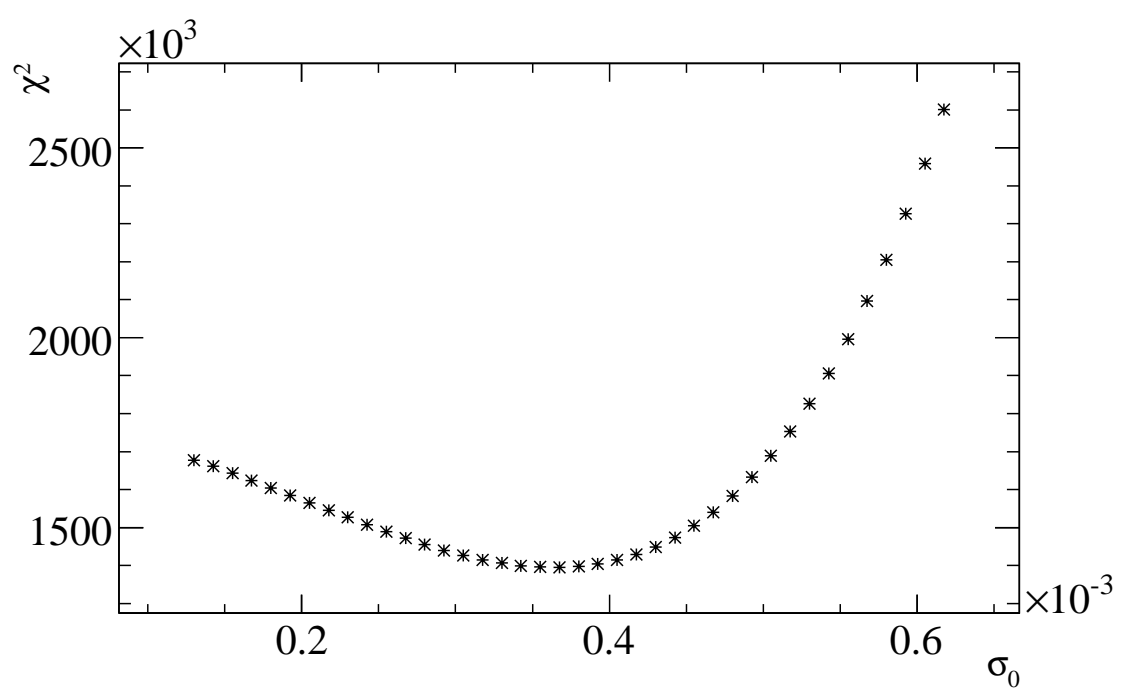

Figure 10.15: $\chi^{2}$ contour for $\sigma_{0}$ in radians.

prediction falls back in line with the data. Thus a small change in momentum had a larger affect on the kaon mass than a small change in density ratio.

$\chi^{2}$ contours for the parameters most correlated with the kaon mass are shown in Figures 10.15 - 10.19. Contours are smooth and parabolic in shape near the minimum for each parameter, although the slope is a bit steeper to the right of the minimum for $\sigma_{0}$. There is a slight bump just to the left of the minimum for the kaon mass. It appears as though two different curves come together at this point. This is due to the small inconsistency between the Hamamatsu and R760 peaks, and indicates that there is some systematic difference in the response and/or selection of each PMT type The transition is smooth and did not prevent Minuit from converging to a good minimum. 


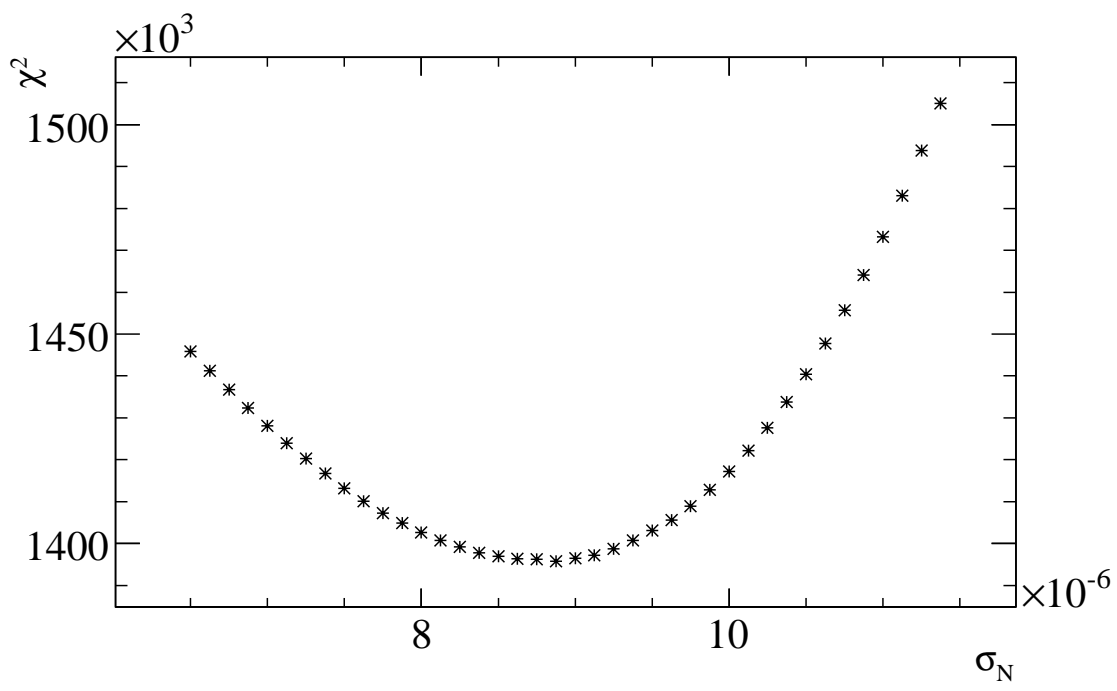

Figure 10.16: $\chi^{2}$ contour for $\sigma_{N}$ in radians.

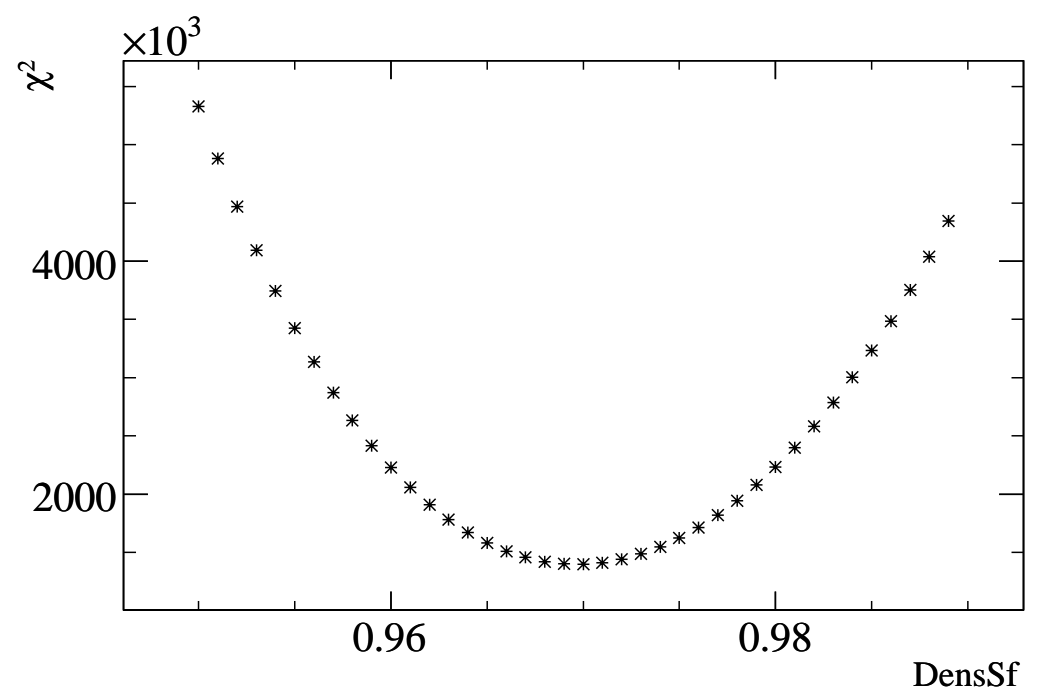

Figure 10.17: $\chi^{2}$ contour for density scaling factor. 


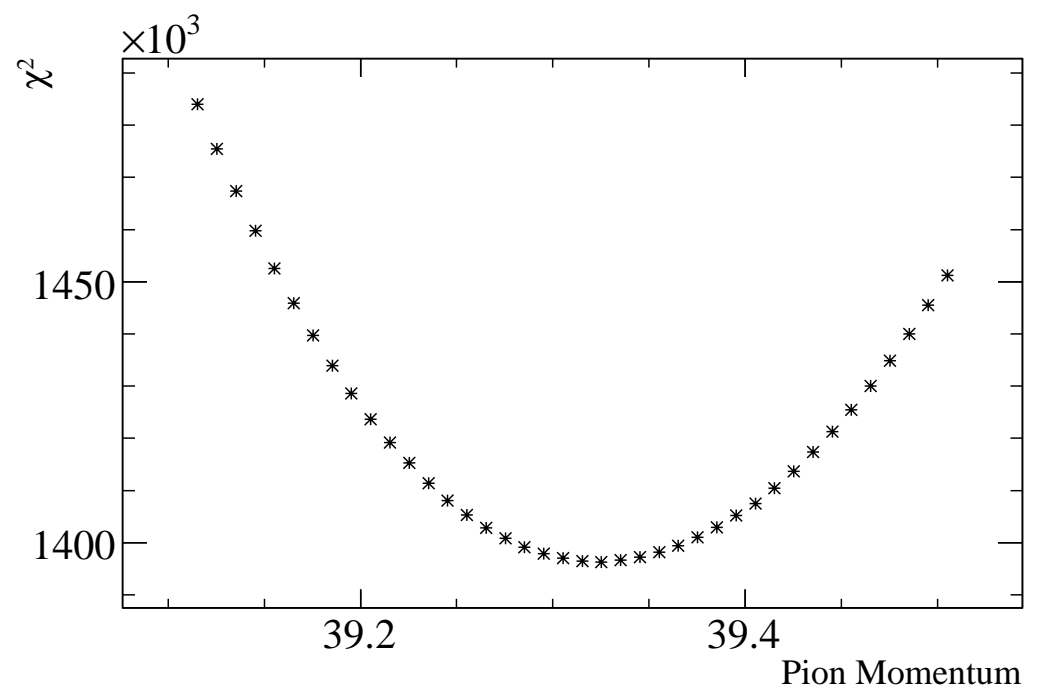

Figure 10.18: $\chi^{2}$ contour for pion momentum in $\mathrm{GeV} / c$.

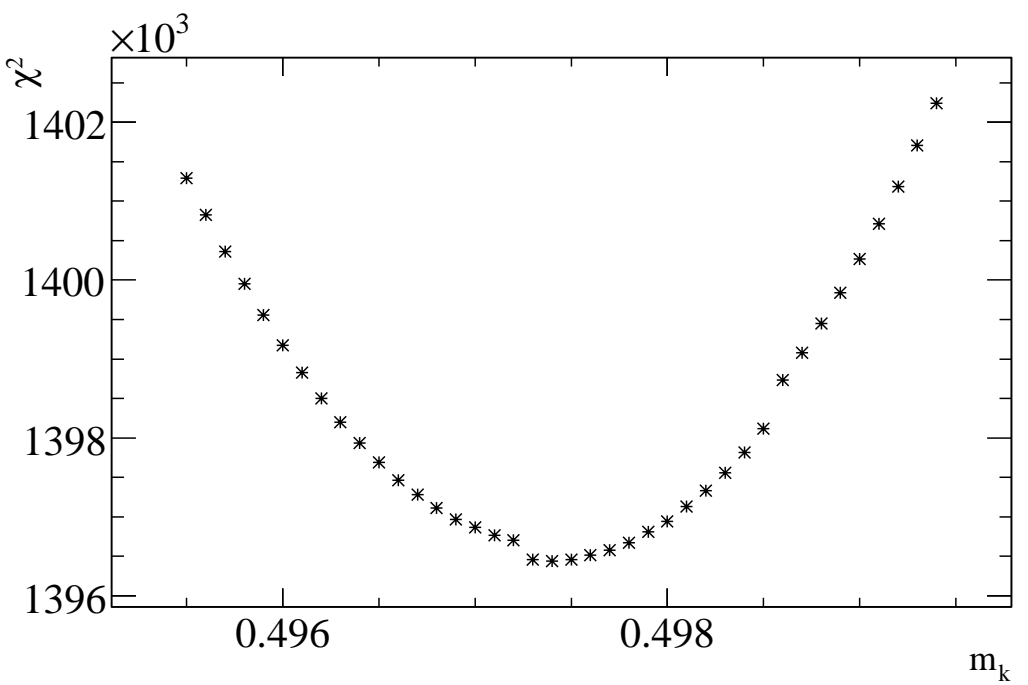

Figure 10.19: $\chi^{2}$ contour for kaon mass in $\mathrm{GeV} / c^{2}$. 

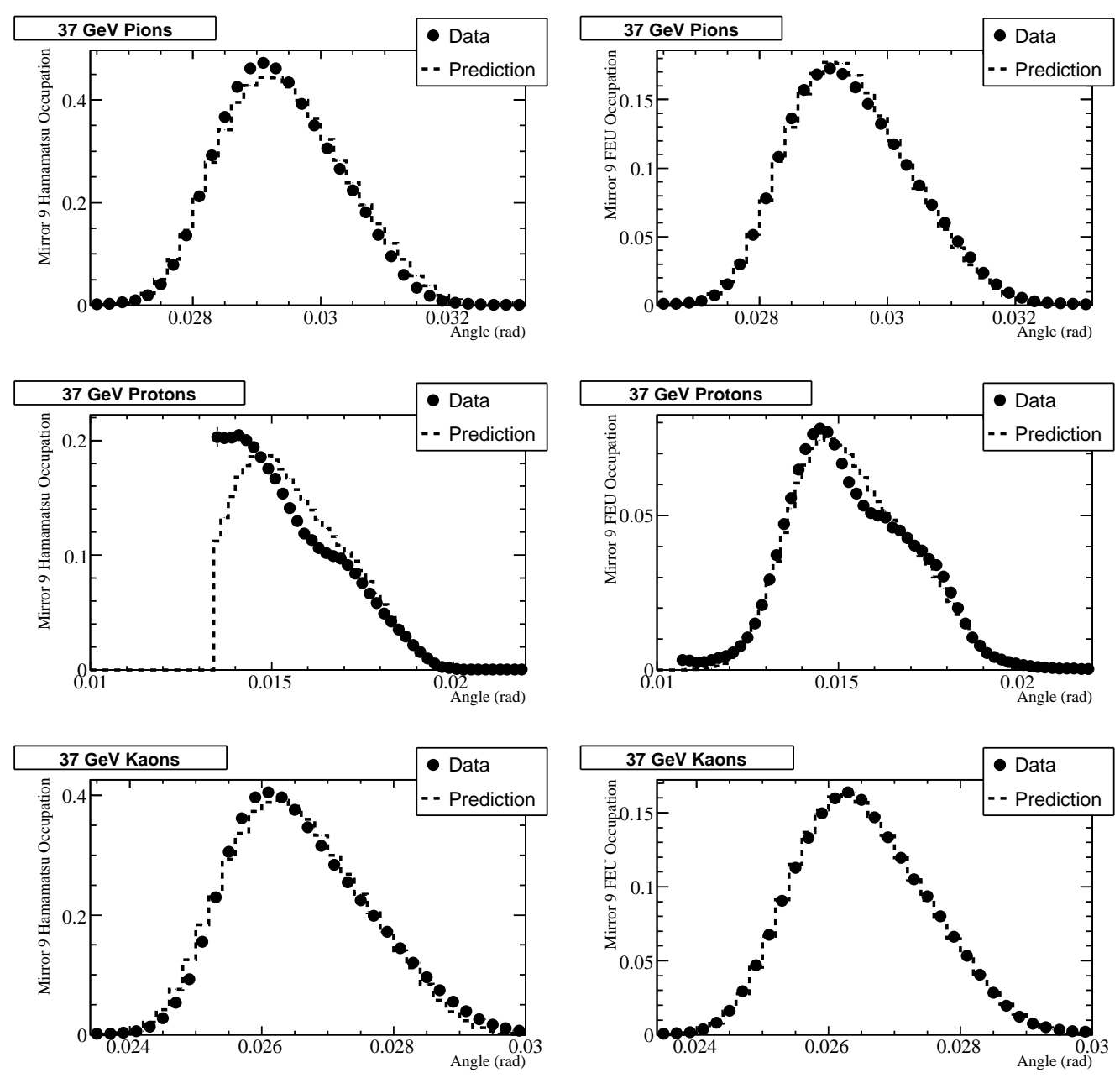

Figure 10.20: Best fit of predicted to measured PMT occupancy for $37 \mathrm{GeV}$ data set, mirror 9 only. Upper Row: Pions. Middle Row: Protons. Lower Row: Kaons. Left Column: Hamamatsu. Right Column: FEU. 

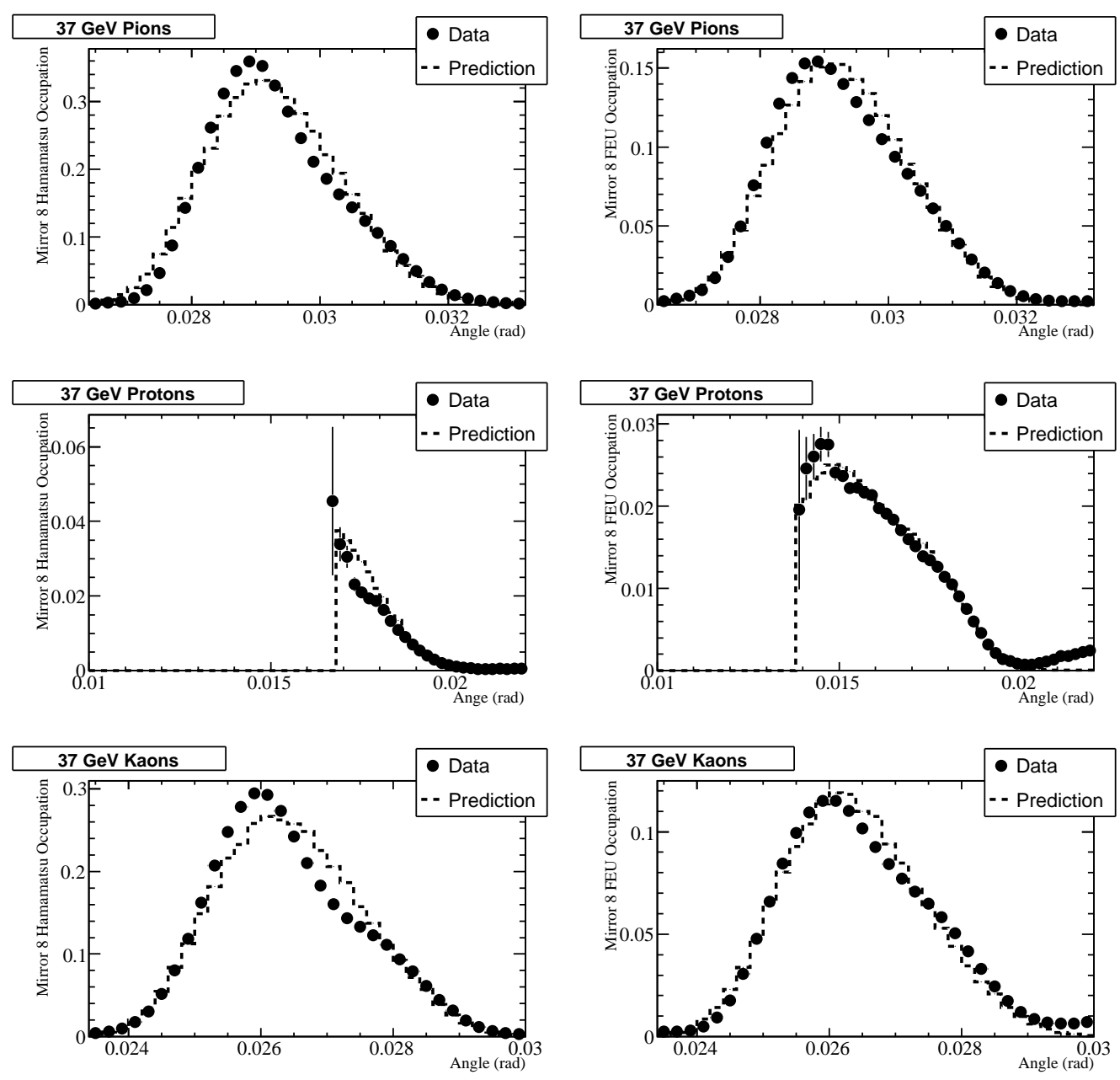

Figure 10.21: Best fit of predicted to measured PMT occupancy for $37 \mathrm{GeV}$ data set, mirror 8 only. Upper Row: Pions. Middle Row: Protons. Lower Row: Kaons. Left Column: Hamamatsu. Right Column: FEU. 

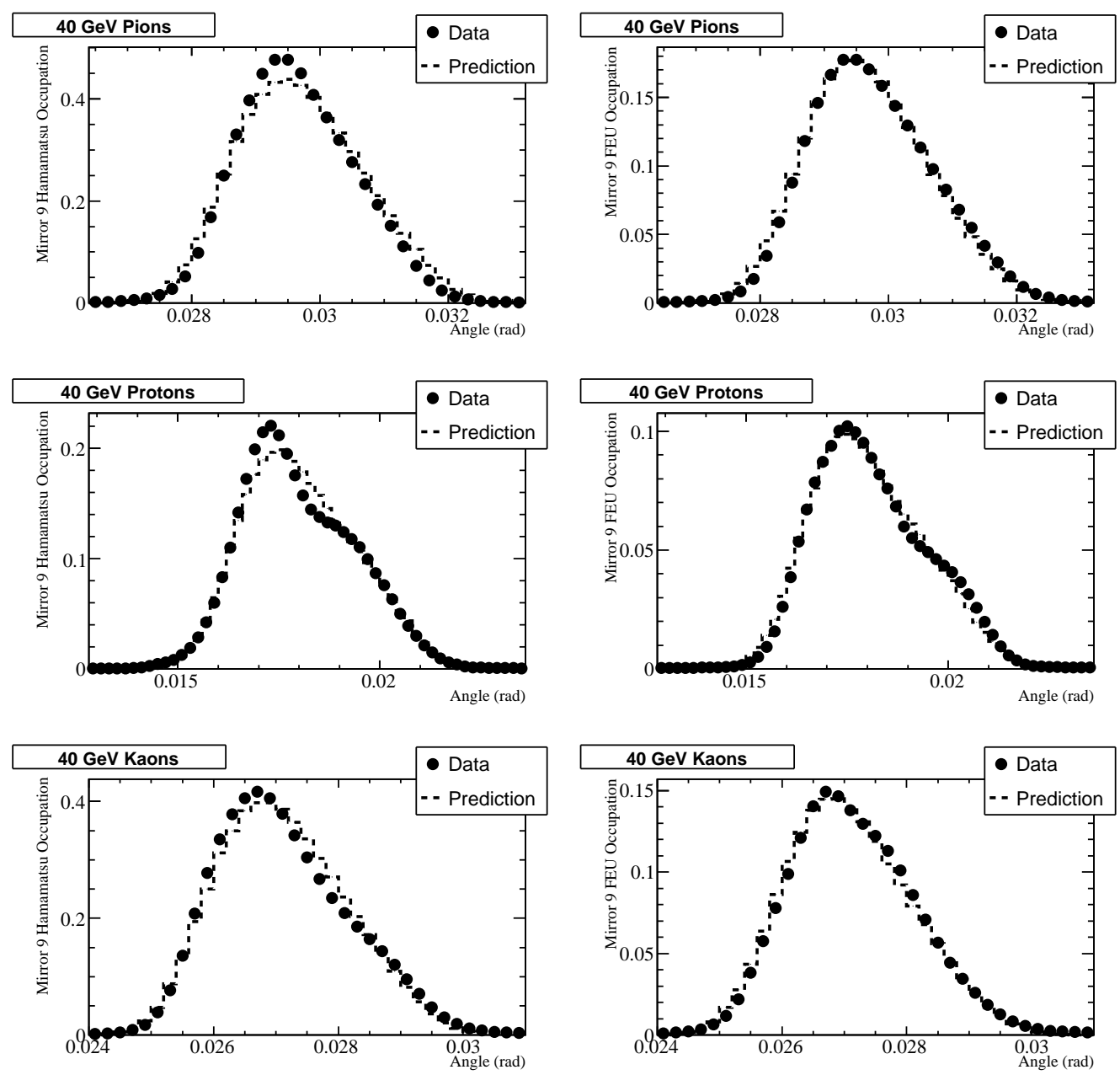

Figure 10.22: Best fit of predicted to measured PMT occupancy for $40 \mathrm{GeV}$ data set, mirror 9 only. Upper Row: Pions. Middle Row: Protons. Lower Row: Kaons. Left Column: Hamamatsu. Right Column: FEU. 

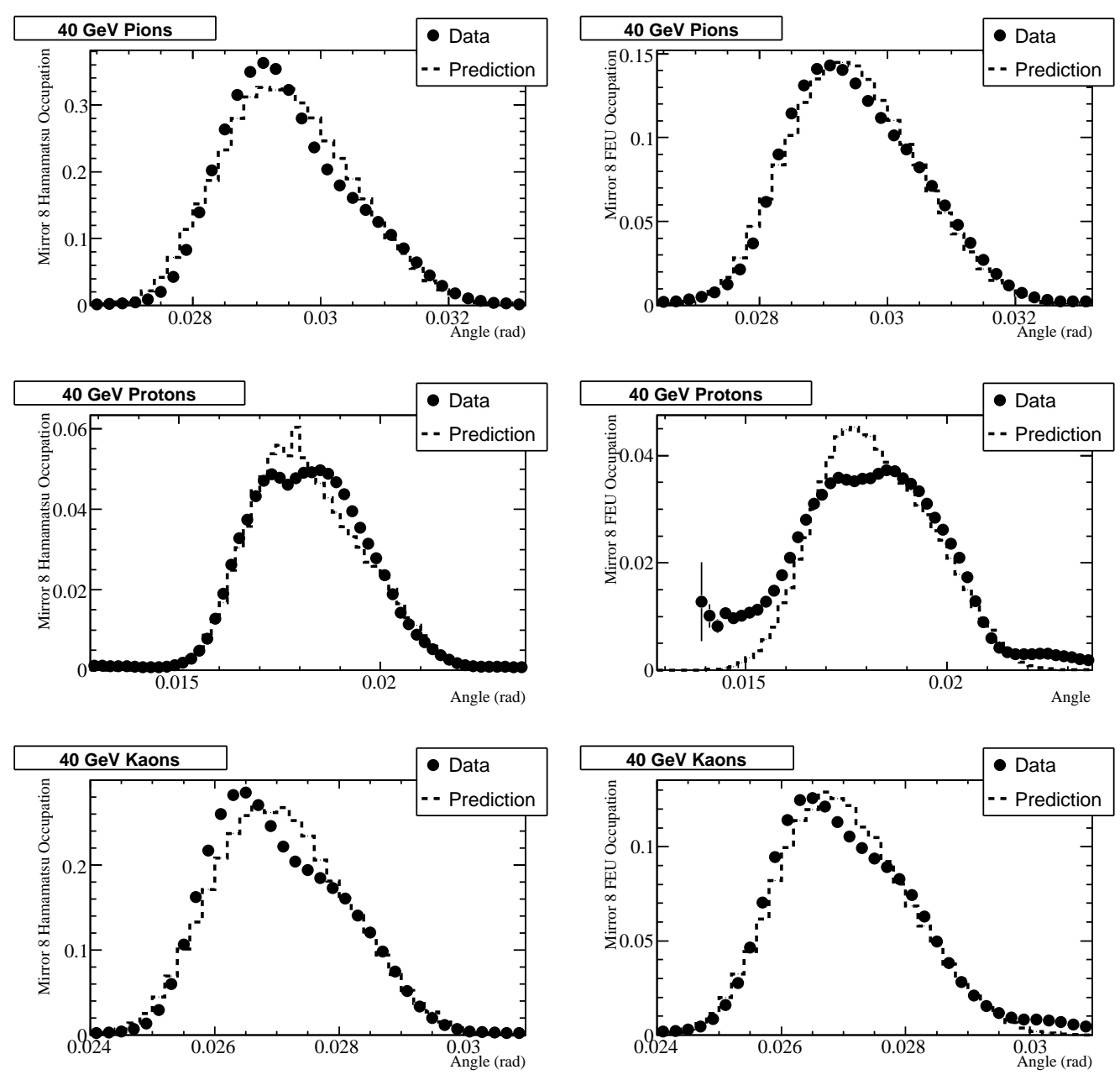

Figure 10.23: Best fit of predicted to measured PMT occupancy for $40 \mathrm{GeV}$ data set, mirror 8 only. Upper Row: Pions. Middle Row: Protons. Lower Row: Kaons. Left Column: Hamamatsu. Right Column: FEU. 

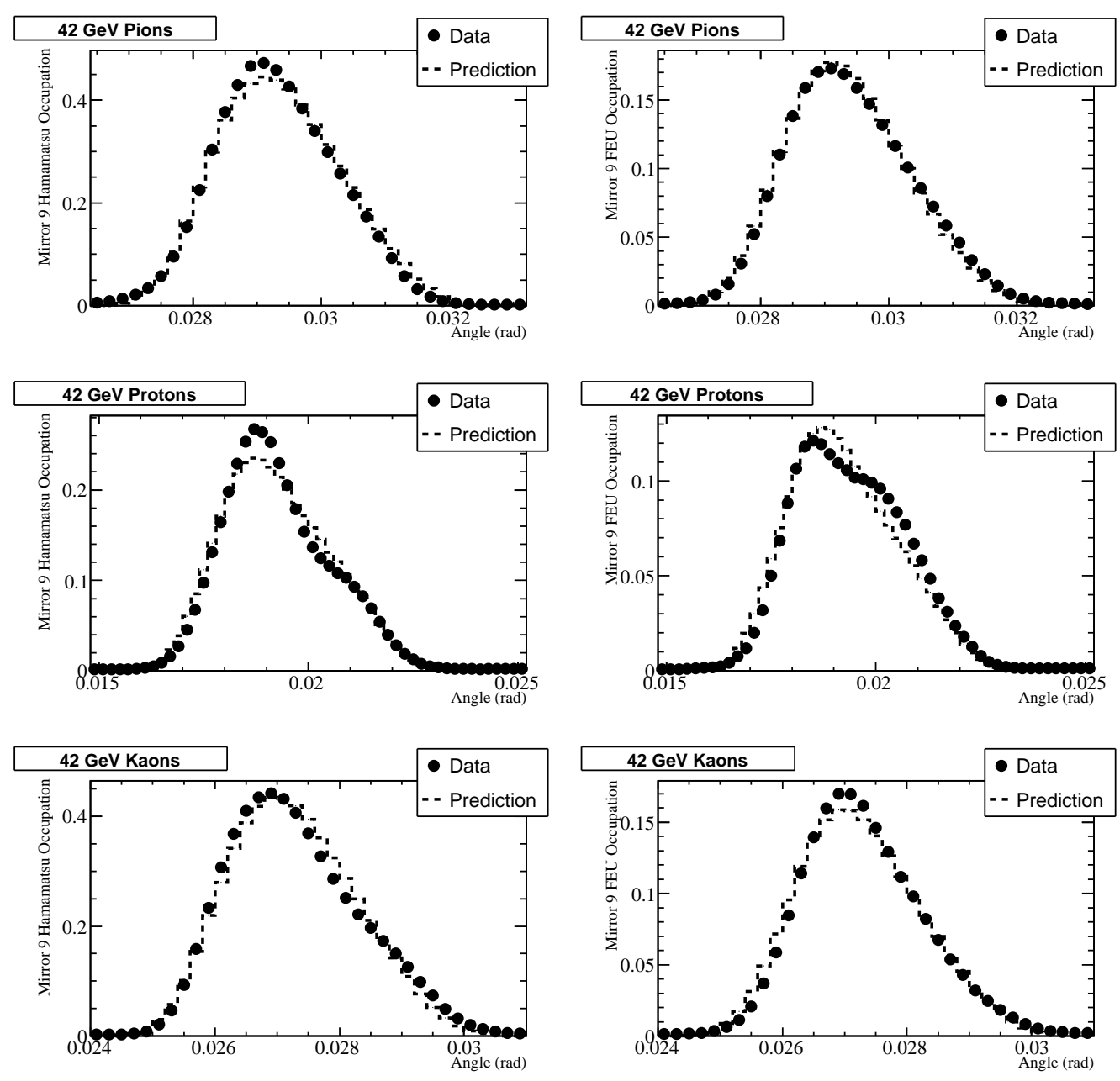

Figure 10.24: Best fit of predicted to measured PMT occupancy for $42 \mathrm{GeV}$ data set, mirror 9 only. Upper Row: Pions. Middle Row: Protons. Lower Row: Kaons. Left Column: Hamamatsu. Right Column: FEU. 

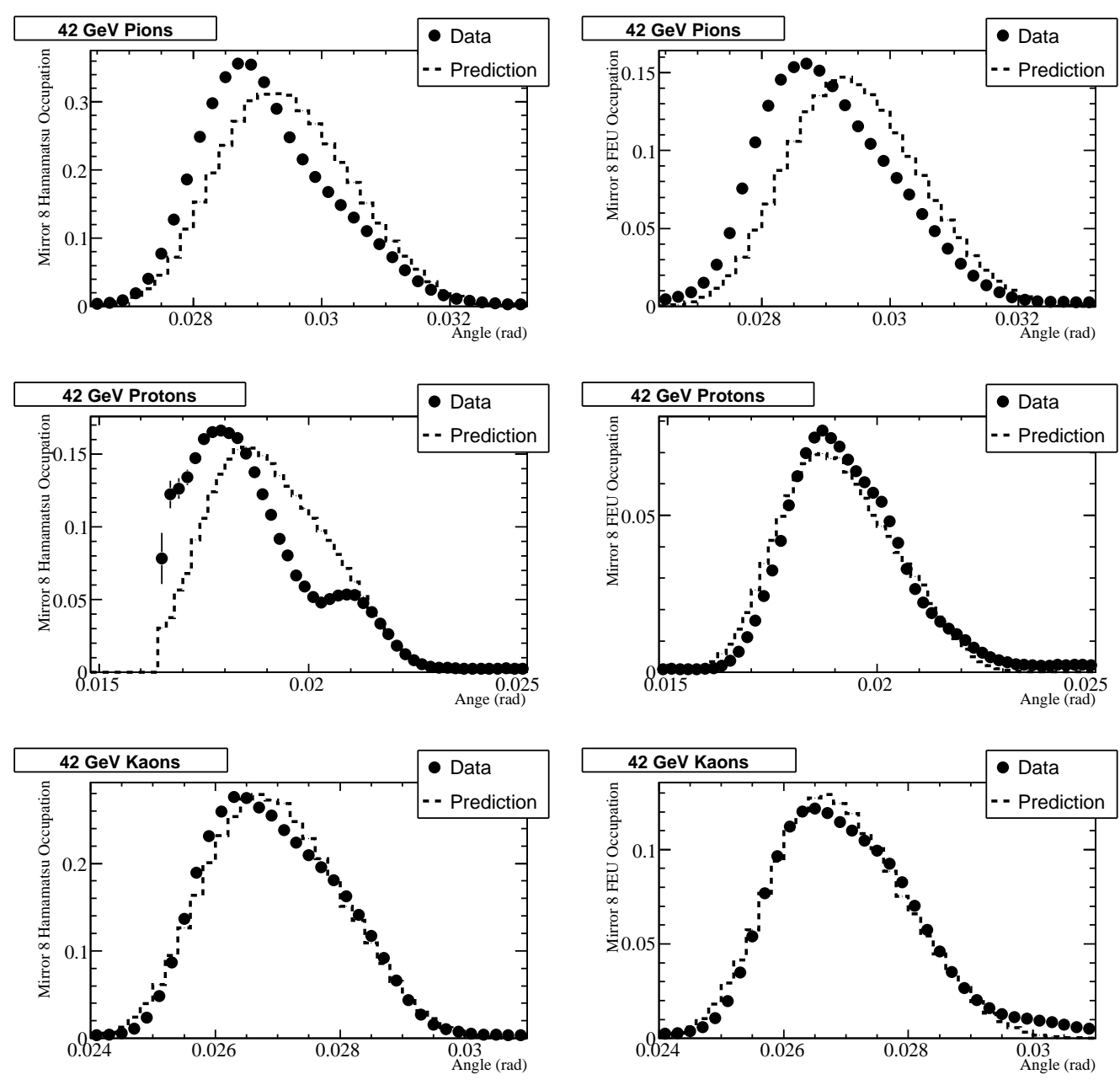

Figure 10.25: Best fit of predicted to measured PMT occupancy for $42 \mathrm{GeV}$ data set, mirror 8 only. Upper Row: Pions. Middle Row: Protons. Lower Row: Kaons. Left Column: Hamamatsu. Right Column: FEU. 

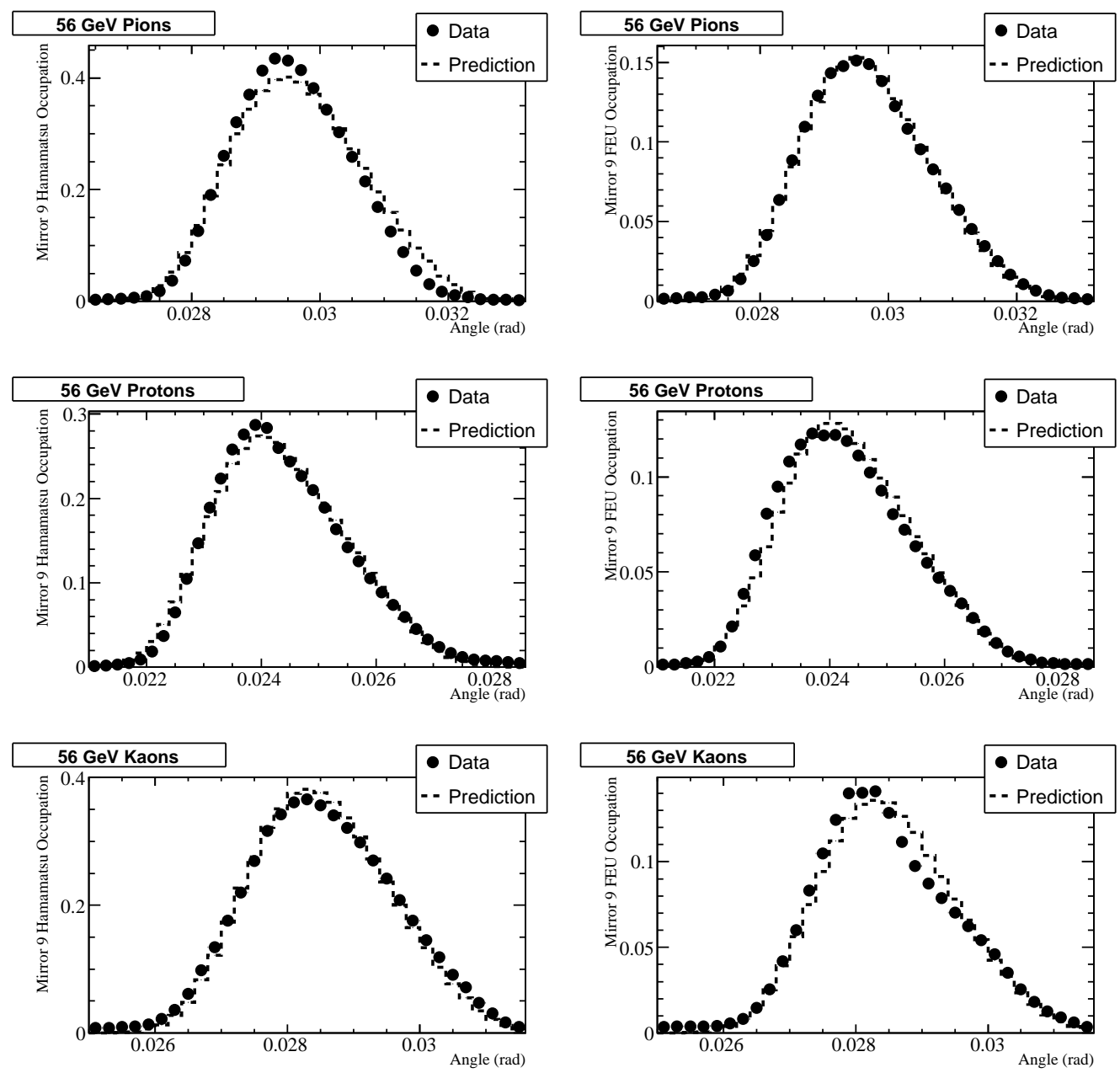

Figure 10.26: Best fit of predicted to measured PMT occupancy for $56 \mathrm{GeV}$ data set, mirror 9 only. Upper Row: Pions. Middle Row: Protons. Lower Row: Kaons. Left Column: Hamamatsu. Right Column: FEU. 

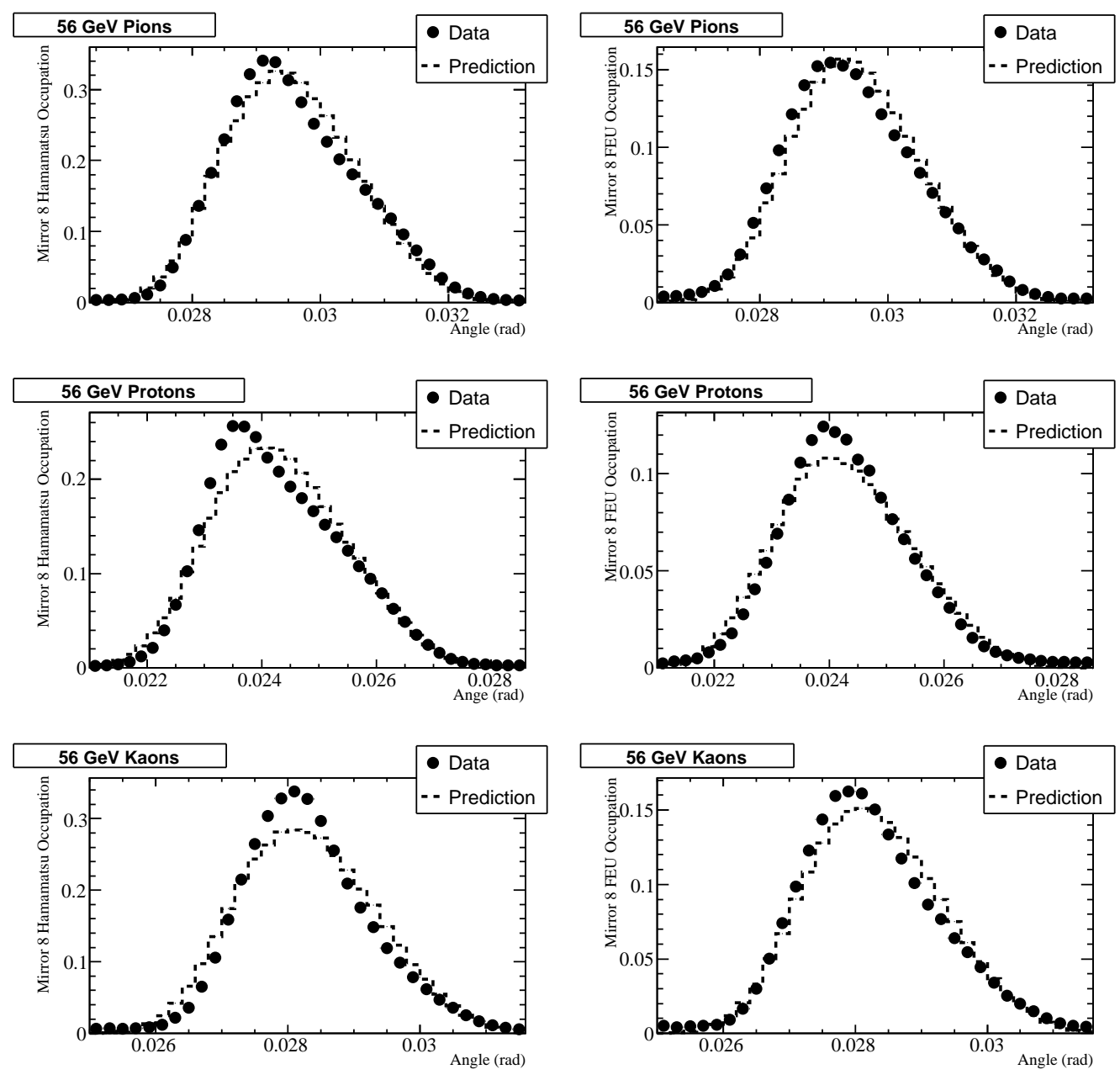

Figure 10.27: Best fit of predicted to measured PMT occupancy for $56 \mathrm{GeV}$ data set, mirror 8 only. Upper Row: Pions. Middle Row: Protons. Lower Row: Kaons. Left Column: Hamamatsu. Right Column: FEU. 

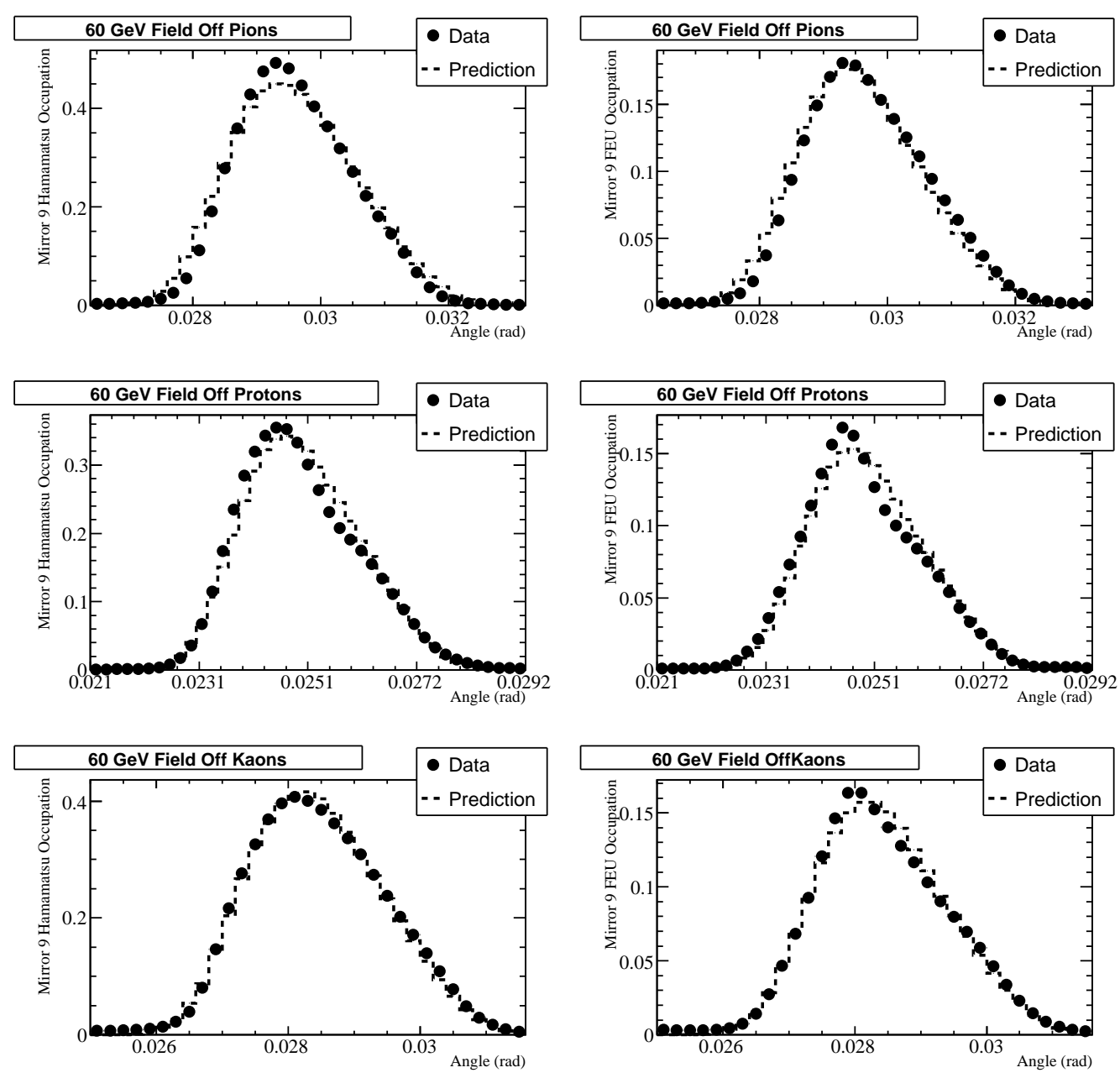

Figure 10.28: Best fit of predicted to measured PMT occupancy for $60 \mathrm{GeV}$ field off data set, mirror 9 only. Upper Row: Pions. Middle Row: Protons. Lower Row: Kaons. Left Column: Hamamatsu. Right Column: FEU. 

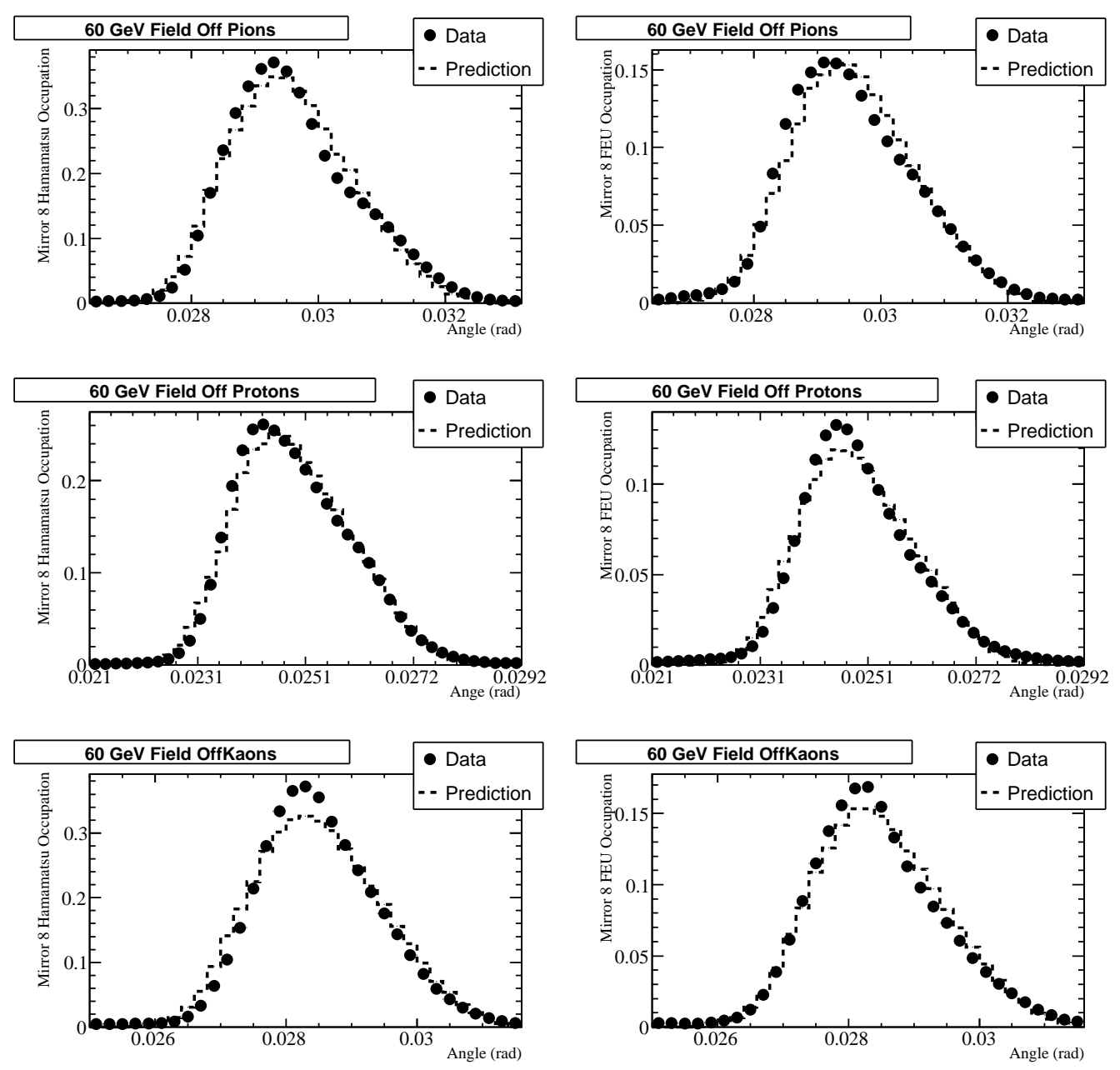

Figure 10.29: Best fit of predicted to measured PMT occupancy for $60 \mathrm{GeV}$ field off data set, mirror 8 only. Upper Row: Pions. Middle Row: Protons. Lower Row: Kaons. Left Column: Hamamatsu. Right Column: FEU. 

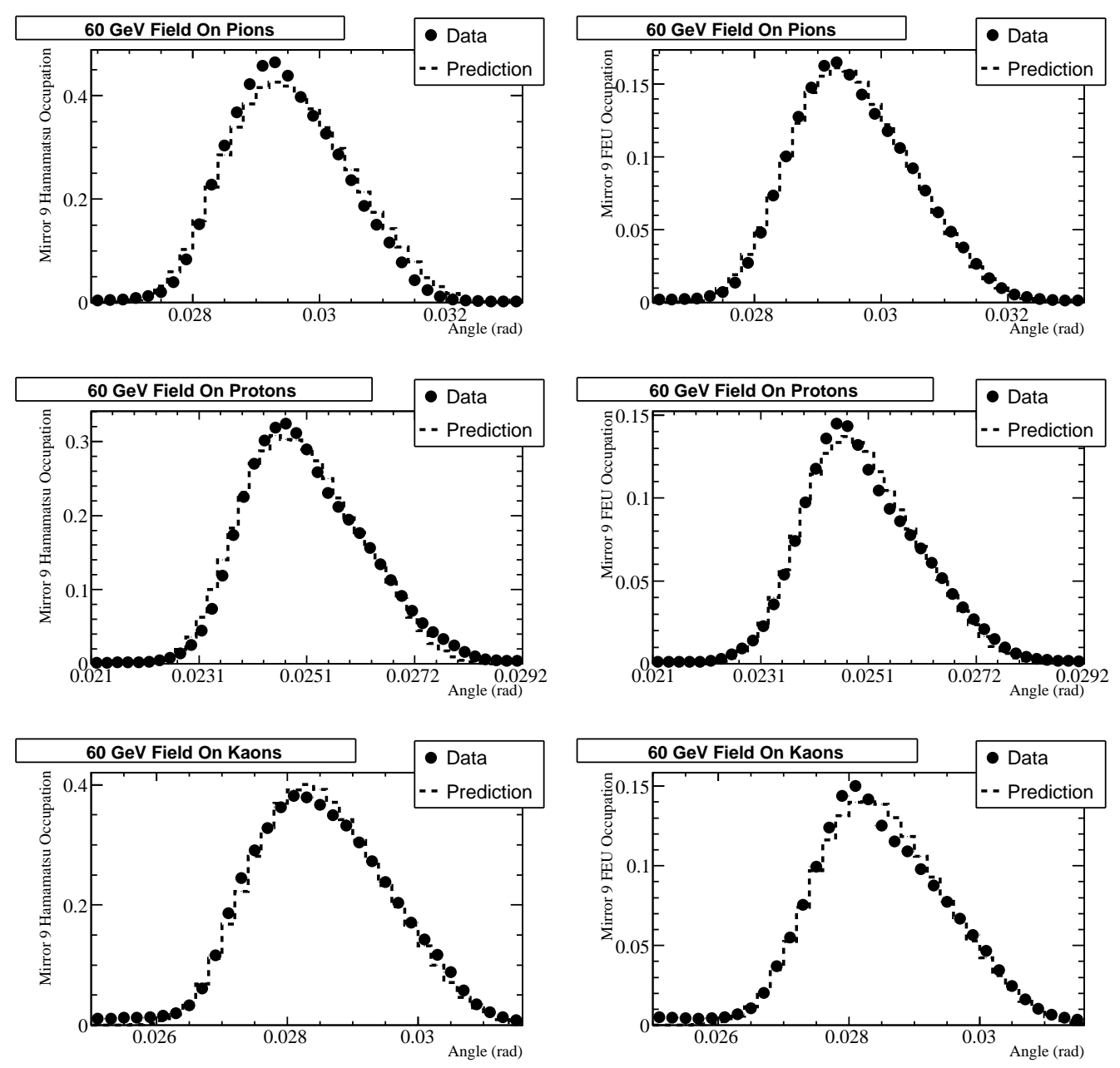

Figure 10.30: Best fit of predicted to measured PMT occupancy for $60 \mathrm{GeV}$ field on data set, mirror 9 only. Upper Row: Pions. Middle Row: Protons. Lower Row: Kaons. Left Column: Hamamatsu. Right Column: FEU. 

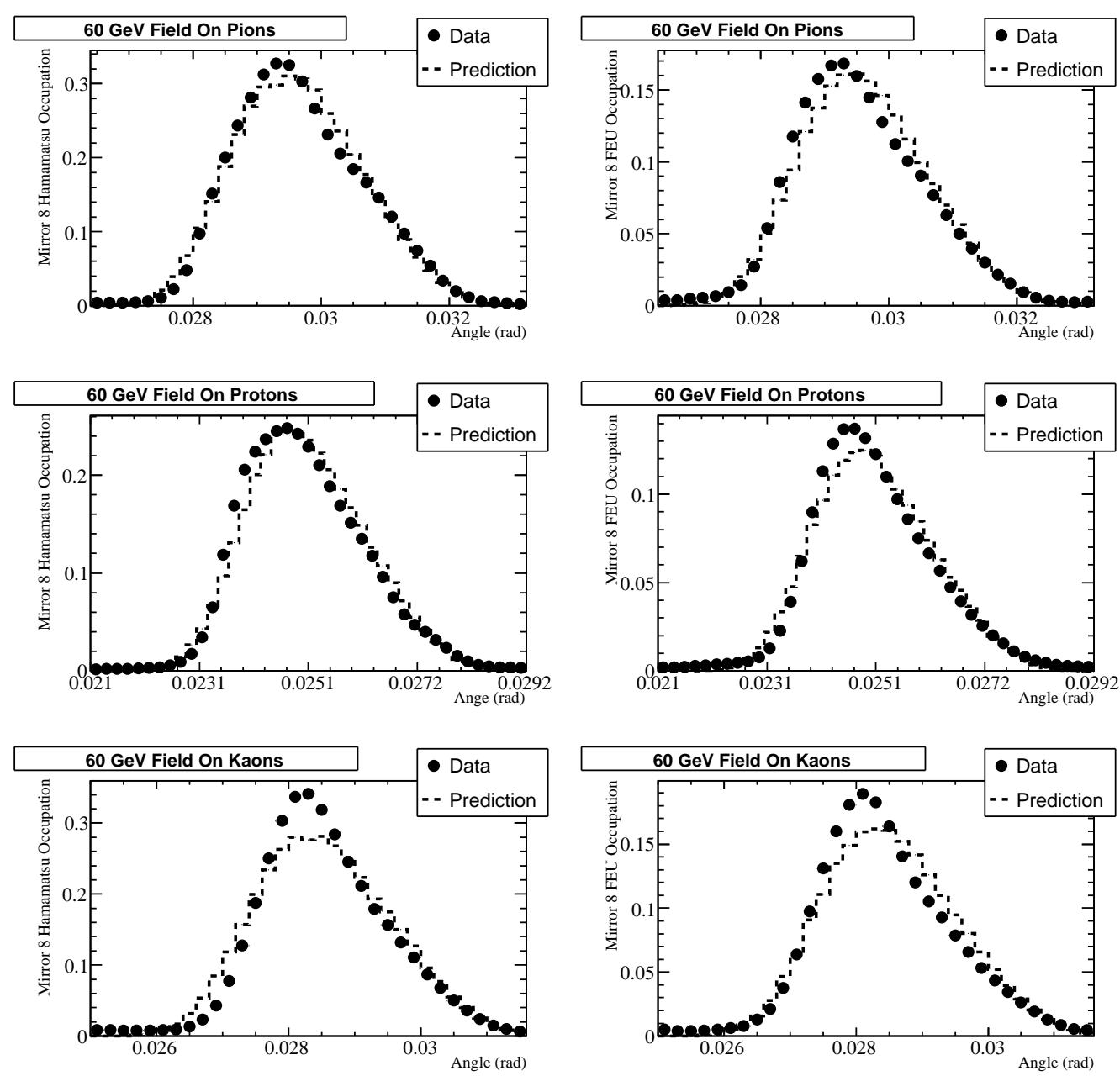

Figure 10.31: Best fit of predicted to measured PMT occupancy for $60 \mathrm{GeV}$ field on data set, mirror 8 only. Upper Row: Pions. Middle Row: Protons. Lower Row: Kaons. Left Column: Hamamatsu. Right Column: FEU. 
Fit results with uncertainties from Minuit are shown in Table 10.8. Uncertainties after error bar scaling are on the order of 3,000-12,000 ppm for low momentum sets, and 10,000-30,000 ppm for high momentum sets. Before scaling, the statistical errors were on the order of 50-200 ppm for low momentum sets and several hundred ppm for the high momentum sets. Scaling is a way to estimate systematic uncertainties, which are two to three orders of magnitude higher. The statistical uncertainty of the measurement is insignificant compared to systematics.

\subsubsection{Systematic Uncertainites}

Error bar scaling provides an estimate of the size of the overall systematic error. Other factors in addition to good tube selection and tube response contribute to the total error: ring center prediction; index of refraction; central momentum; width of momentum distribution. Uncertainty in ring center prediction would effectively widen the photoelectron distribution, so this contribution was estimated from the smearing parameters $\sigma_{0}$ and $\sigma_{N}$. The error in refractive index was estimated from the density scaling and offset parameters, as well the level of air contamination.

Estimates of how much each source contributes were made by evaluating first derivates at a point near the minimum and using the unscaled errors for 

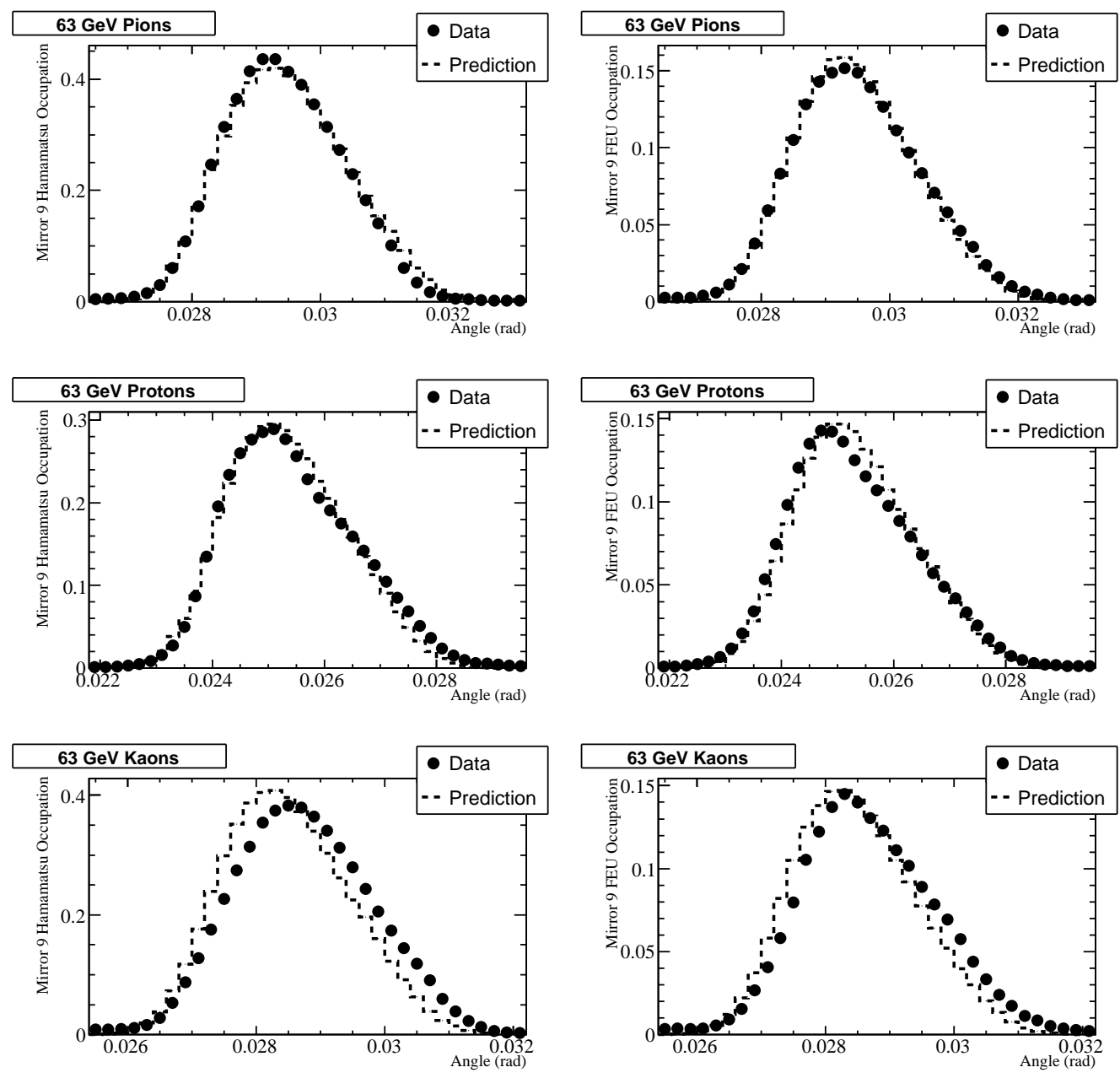

Figure 10.32: Best fit of predicted to measured PMT occupancy for $63 \mathrm{GeV}$ data set, mirror 9 only. Upper Row: Pions. Middle Row: Protons. Lower Row: Kaons. Left Column: Hamamatsu. Right Column: FEU. 

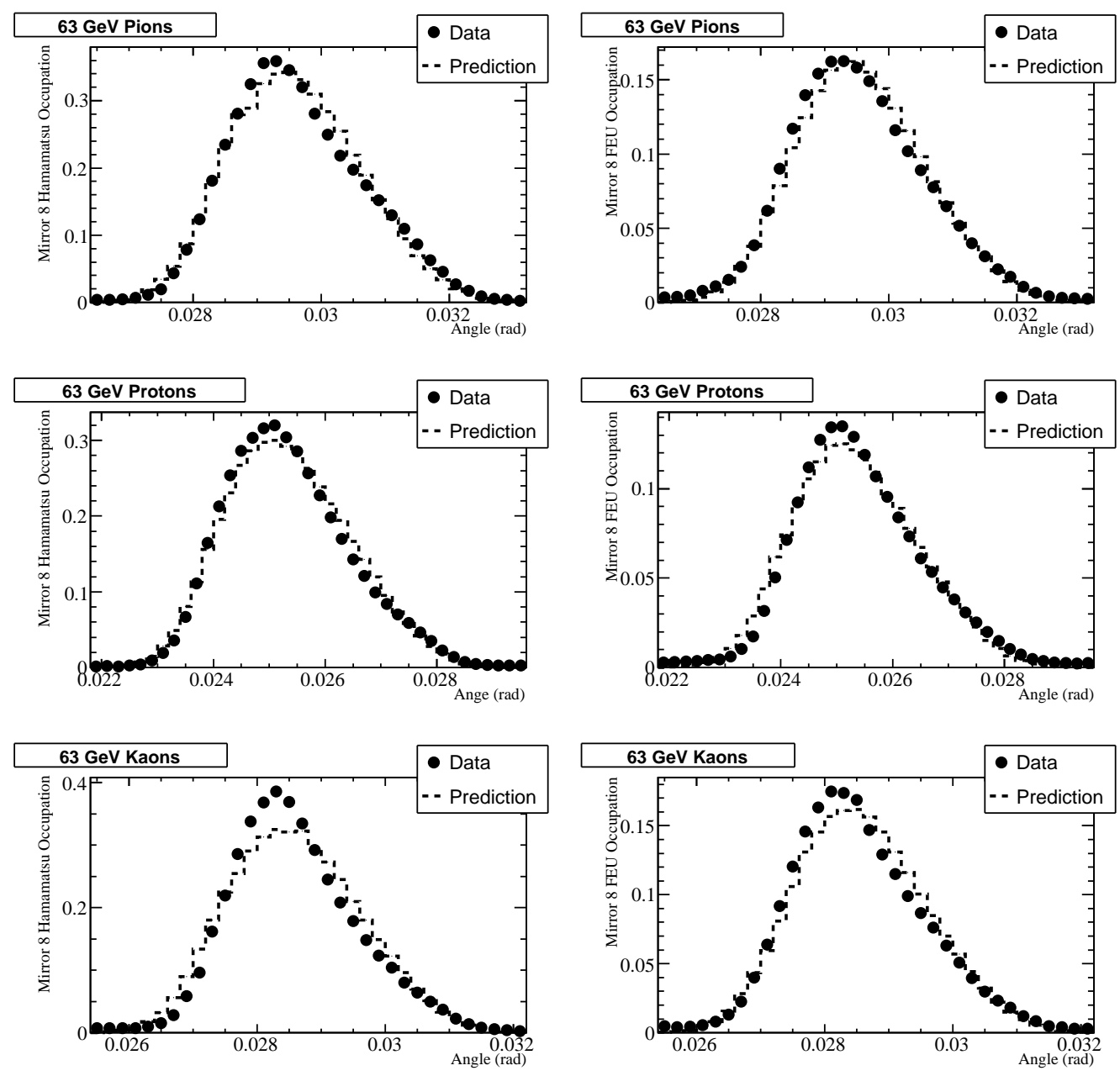

Figure 10.33: Best fit of predicted to measured PMT occupancy for $63 \mathrm{GeV}$ data set, mirror 8 only. Upper Row: Pions. Middle Row: Protons. Lower Row: Kaons. Left Column: Hamamatsu. Right Column: FEU. 


\begin{tabular}{l|cc}
\hline \hline Data Set & $\begin{array}{c}\text { Kaon Mass } \\
(\mathrm{MeV})\end{array}$ & $\begin{array}{c}\text { Uncertainty } \\
(\mathrm{MeV})\end{array}$ \\
\hline $37 \mathrm{GeV}$ Mirror 8 & 493.3 & 1.5 \\
$37 \mathrm{GeV}$ Mirror 9 & 490.5 & 2.0 \\
$40 \mathrm{GeV}$ Mirror 8 & 487.1 & 5.1 \\
$40 \mathrm{GeV}$ Mirror 9 & 497.5 & 4.2 \\
$42 \mathrm{GeV}$ Mirror 8 & 510.8 & 6.3 \\
$42 \mathrm{GeV}$ Mirror 9 & 477.8 & 4.5 \\
$56 \mathrm{GeV}$ Mirror 8 & 487.9 & 4.5 \\
$56 \mathrm{GeV}$ Mirror 9 & 469 & 13 \\
60 GeV Field Off Mirror 8 & 481.3 & 8.2 \\
$60 \mathrm{GeV}$ Field Off Mirror 9 & 499.7 & 8.7 \\
$60 \mathrm{GeV}$ Field On Mirror 8 & 491 & 32 \\
$60 \mathrm{GeV}$ Field On Mirror 9 & 477 & 11 \\
$63 \mathrm{GeV}$ Mirror 8 & 485 & 14 \\
$63 \mathrm{GeV}$ Mirror 9 & 489.6 & 8.5 \\
\hline All 40 GeV Sets & 491.9 & 1.1 \\
All 60 GeV Sets & 486.7 & 3.0 \\
\hline Final Result & 491.3 & 1.7 \\
\hline \hline
\end{tabular}

Table 10.8: Summary of kaon mass fit results. All of the low momentum sets were fit for a single mass value except for the $42 \mathrm{GeV}$ mirror 8 set. The same was done for the high momentum sets. These results were fit to obtain the final result, with the errors scaled so that $\chi^{2}=1$. 


\begin{tabular}{l|ccc}
\hline \hline & Error & $\partial \chi^{2} / \partial x$ & $\partial m_{K} / \partial x$ \\
\hline$\sigma_{0}$ & $3.12 \times 10^{-7} \mathrm{mrad}$ & 74664 & -0.053 \\
$\sigma_{N}$ & $8.67 \times 10^{-9} \mathrm{mrad}$ & 37882 & -0.027 \\
DensSF & $1.24 \times 10^{-5}$ & $6.74 \times 10^{6}$ & -4.8 \\
DensOS & $1.18 \times 10^{-5}$ & $7.02 \times 10^{6}$ & -5.0 \\
$\sigma_{P}$ & $0.016 \mathrm{GeV}$ & -229 & $1.63 \times 10^{-4}$ \\
AirFrac & $0.99 \mathrm{ppm}$ & -749 & $5.30 \times 10^{-4}$ \\
$P_{\pi}$ & $0.00078 \mathrm{GeV}$ & 15494 & -0.011 \\
\hline \hline
\end{tabular}

Table 10.9: Parameter uncertainties and first derivative estimates for $40 \mathrm{GeV}$ mirror 9 data set. $\partial \chi^{2} / \partial m_{K}=-1.4 \times 10^{6}$.

\begin{tabular}{l|c}
\hline \hline Source & Contribution \\
\hline Ring Center & $2 \mathrm{ppm}$ \\
Refractive Index & $450 \mathrm{ppm}$ \\
Momentum Width & $4 \mathrm{ppm}$ \\
Central Momentum & $40 \mathrm{ppm}$ \\
Tube Selection/Response & 8420 \\
\hline Total & $8440 \mathrm{ppm}$ \\
\hline \hline
\end{tabular}

Table 10.10: Breakdown of systematic error estimates for $40 \mathrm{GeV}$ mirror 9 data set. The total is the sum in quadrature of each contribution.

each parameter:

$$
\sigma_{m_{K}}^{2}=\sum_{i=1}^{n}\left[\left(\frac{\partial m_{K}}{\partial x_{i}}\right)^{2} \sigma_{x_{i}}^{2}+\sum_{j \neq i} \operatorname{cov}\left(x_{i}, x_{j}\right) \frac{\partial m_{K}}{\partial x_{i}} \frac{\partial m_{K}}{\partial x_{j}}\right]
$$

where $n$ is the total number of parameters and $\operatorname{cov}(x, y)$ is the covariance between parameters $x$ and $y$. The covariance is related to the correlation coefficient via: $\operatorname{cov}(x, y)=\sigma_{x} \sigma_{y} \operatorname{corr}(x, y)$.

Table 10.10 shows the systematic error estimates for the $40 \mathrm{GeV}$ mirror 9 
data set for each parameter, with the remainder attributed to selecting good tubes and understanding of tube response. This was the dominate source of error, contributing at the $8400 \mathrm{ppm}$ level The next largest contribution was from the refractive index at $450 \mathrm{ppm}$, followed by the central momentum at $40 \mathrm{ppm}$ Ring center prediction and momentum width did not contribute significantly at $2 \mathrm{ppm}$ and $4 \mathrm{ppm}$ respectively.

\subsection{Final Result and Conclusions}

Mass results for low momentum data sets are plotted in Figure 10.34 with $42 \mathrm{GeV}$ mirror 8 excluded. They are fit for a single mass value. The result is $491.9 \pm 1.1 \mathrm{MeV}$. The $\chi^{2}$ is 9.754 with a probability for 4 degrees of freedom of $4.5 \%$. If the $42 \mathrm{GeV}$ mirror 8 result had been included, the $\chi^{2}$ probability drops to $0.03 \%$.

The same was done for the high momentum sets (Figure 10.35). The result is $486.7 \pm 3.0 \mathrm{MeV}$. The $\chi^{2}$ is 5.426 with a probability for 7 degrees of freedom of $61 \%$.

These two results are plotted and fit in Figure 10.36, for a kaon mass of $491.3 \pm 1.0 \mathrm{MeV}$. The $\chi^{2}$ is 2.625 with a probability for 1 degree of freedom

of $10.5 \%$. The final error is scaled by $\sqrt{\chi^{2}}$ for a conservative estimate of 1.7 MeV. The final measured value for the charged kaon mass is $491.3 \pm 1.7$ $\mathrm{MeV}$. This is within $1.4 \sigma$ of the PDG value. An improvement by a factor of 35 in the precision would make this technique competitive for resolving 


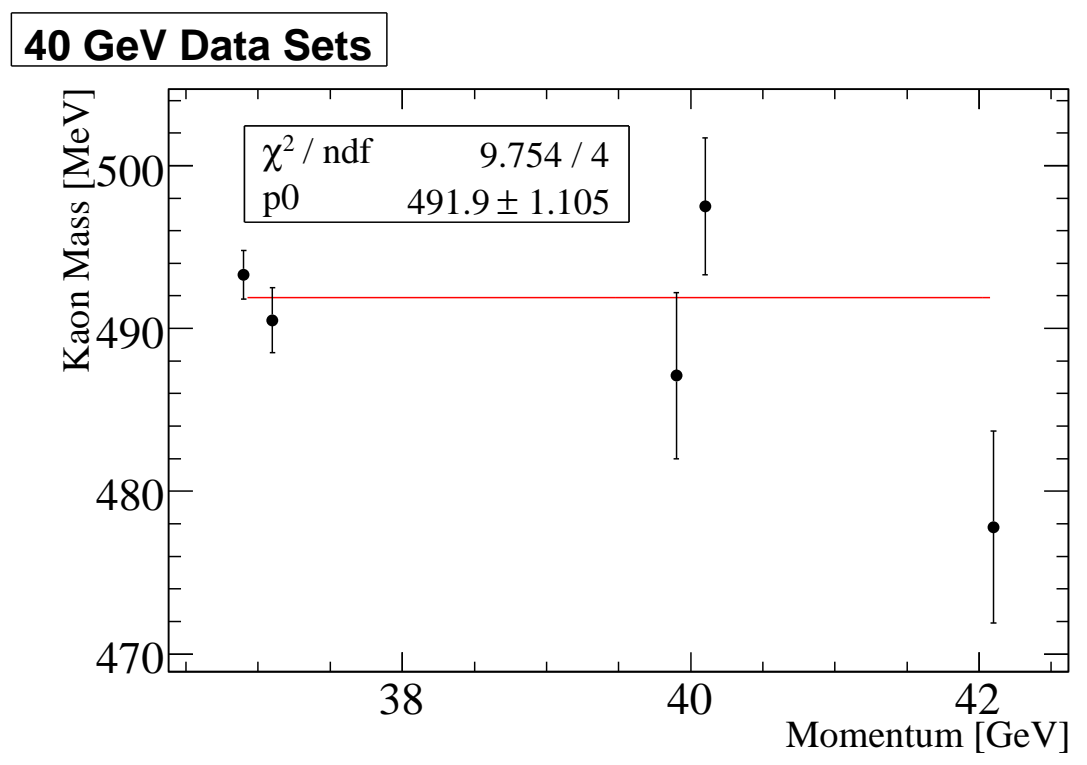

Figure 10.34: Fit to a single mass value for results of low momentum data sets. Points on the left at a particular momentum are for mirror 8 and those on the right are for mirror 9 . 


\section{$60 \mathrm{GeV}$ Data Sets}

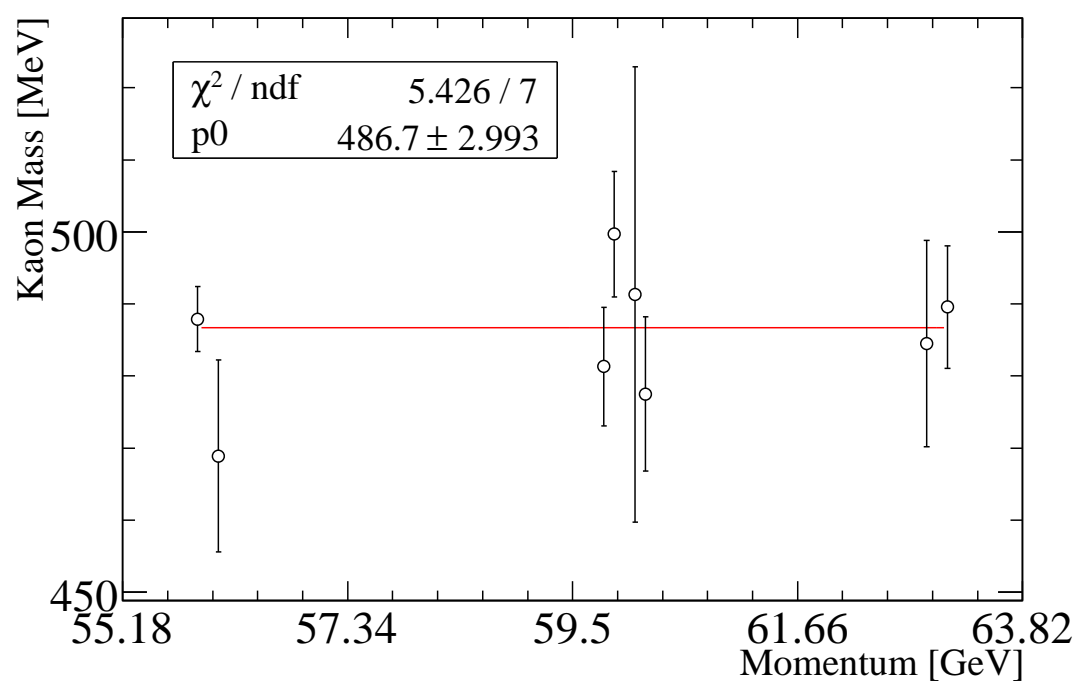

Figure 10.35: Fit to a single mass value for results of high momentum data sets. The points near $60 \mathrm{GeV}$ are from the left: field on mirror 8, field on mirror 9, field off mirror 8, field off mirror 9 . For $56 \mathrm{GeV}$ and $63 \mathrm{GeV}$, the leftmost point is mirror 8 and the right most point is mirror 9 . 


\section{Final Result}

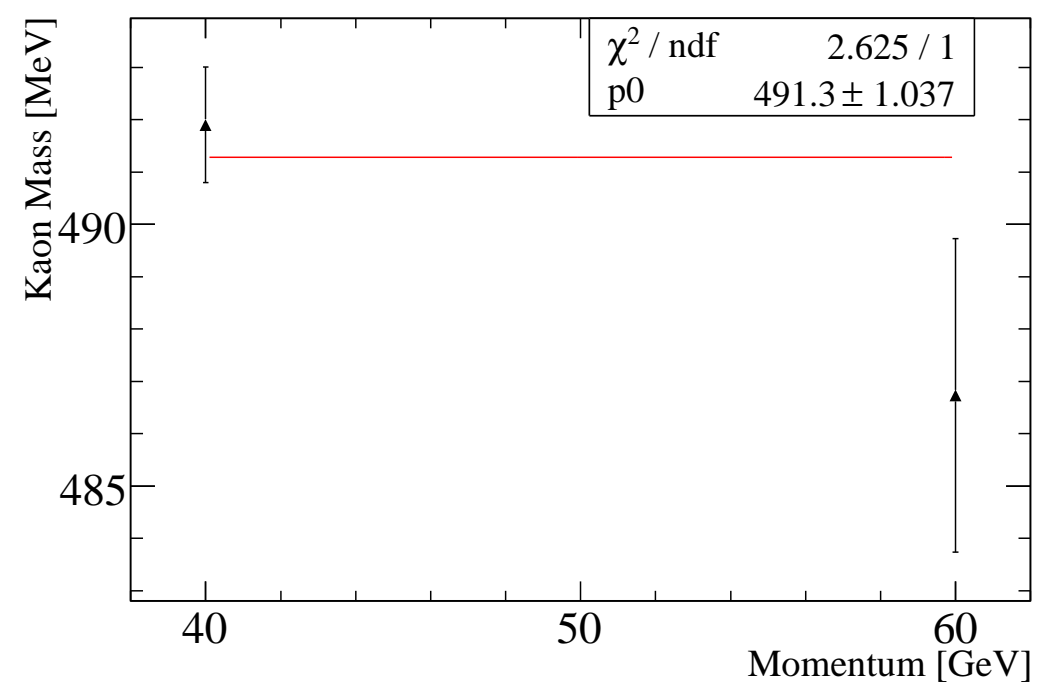

Figure 10.36: Final mass result. Points are fit results for $40 \mathrm{GeV}$ data sets and $60 \mathrm{GeV}$ data sets respectively. The error bars are scaled so that $\chi^{2} / \mathrm{ndf}$ $=1$ for a final uncertainty of $1.7 \mathrm{MeV}$.

ambiguity in the X-ray data set.

\subsubsection{Suggestions for Improvement}

The most important consideration for improving this technique is to accurately measure the response of each channel in the photodector, including efficiency and noise rate. A readout system that can handle the data rate would eliminate the problem of hot columns seen in this data set. A much more finely segmented photo-detector using HPDs with ADC information would provide better resolution of the light distribution and allow the num- 
ber of photoelectrons to be counted, as opposed to recording an "on" or "off" state for each channel. These improvements would reduce the dominant systematic uncertainties.

Once the response of the photo-detector is well known, other sources of systematic error would dominate and would need to be controlled more precisely and studied more carefully. A better understanding of the optical properties of the radiator would be necessary to compete with the precision of the X-ray measurements. Aerogel maintained at a uniform temperature would have a stable refractive index and a higher light yield per unit of path length. Measuring the refractive index in step of $1 \mathrm{~nm}$ would improve predictions of the angular light distribution.

Production spectra could be compared using several different hadron production models in conjunction with a detailed simulation of the beamline, along with direct measurements of the momentum on a track-by-track basis. It would also be helpful to have a single focusing mirror, so that Cherenkov light is not divided between different mirrors with different focal lengths. A Monte Carlo simulation could be used to study the effects of uncertainties in all of these parameters, including ring center prediction.

With a precise momentum measurement for each track, the analysis could be extended beyond fitting photoelectron distributions vs. angle. These would provide the first step of calibrating parameters using the proton and pion data. Then, for each event, a likelihood function could be constructed using measured and predicted photoelectrons. A mass result could be ob- 
tained for each track using the method of maximum likelihood, and the distributions for each particle species fit to obtain the best values. If the proton and pion results did not agree with accepted values within errors, then more work studying the systematics would be needed. 


\section{Bibliography}

[1] http://mathworld.wolfram.com/QuarticEquation.html.

[2] Handbook of chemistry and physics, $88^{t h}$ edition, 2007. http://www. hbcpnetbase.com/.

[3] G. Backenstoss et al. $K^{-}$mass and $K^{-}$polarizability from kaonic atoms. Phys. Lett. B, 43:431, 1973.

[4] W. Baker et al. Report of the joint MIPP/PPD RICH Panel on the RICH Accident of March 14, 2004. http://ppd.fnal.gov/ experiments/e907/RICH/accident/richPanelReport.pdf, April 2004 .

[5] L.M. Barkov et al. The charged kaon mass measurement. Nucl. Phys. $B, 148: 53,1979$.

[6] A. Bideau-Mehu, Y. Guern, R. Abjean, and A. Johannin-Gilles. Interferometric determination of the refractive index of carbon dioxide in the 
ultraviolet region. Optics Communications, 9(4):432-434, September 1973.

[7] R.A. Burnstein et al. Hypercp: A high-rate spectrometer for the study of charged hyperon and kaon decays. Nucl. Instr. and Meth. A, 541:516$565,2005$.

[8] S.C. Cheng et al. $K^{-}$mass from kaonic atoms. Nucl. Phys. A, 254:381, 1975.

[9] D.C. Christian et al. High rate drift chambers. Nucl. Instr. and Meth. A, 345:62-71, 1994.

[10] SELEX Collaboration. http://fn781a.fnal.gov.

[11] A.S. Denisov et al. New measurement of the mass of the $K^{-}$meson. JEPT Lett., 54:558, 1991.

[12] M. Depalma et al. A system of large multiwire proportional chmabers for a high intensity experiment. Nucl. Instr. and Meth., 216:393-397, 1983.

[13] Eidelman et al. Particle data group. Phys. Lett. B, 1:592, June 2004.

[14] J. Engelfried et al. The e781 (SELEX) RICH detector. Nucl. Instr. and Meth. A, 409:439-442, 1998.

[15] J. Engelfried et al. The RICH detector of the SELEX experiment. Nucl. Instr. and Meth. A, 433:149-152, 1999. 
[16] J. Engelfried et al. The SELEX phototube RICH detector. Nucl. Instr. and Meth. A, 431:53-69, 1999.

[17] A. Fasso' et al. The physics models of FLUKA: status and recent developments. In Computing in High Energy and Nuclear Physics Conference, 2003.

[18] A. Fasso', A. Ferrari, J. Ranft, and P.R. Sala. FLUKA: a multi-particle transport code. CERN 2005-10, INFN/TC_05/11, SLAC-R-773, 2005.

[19] R. Forty and O. Scheider. RICH pattern recognition. LHCb technical note number LHCB/98 040, 1998. http://lhcb.web.cern.ch/ lhcb-rich/html/lhcb_rich_notes.htm.

[20] K.P. Gall et al. Precision measurement of the $K^{-}$and $\sigma^{-}$masses. Phys. Rev. Lett., 60:186, 1988.

[21] Welfried Heller. The determination of refractive indices of colloidal particles by means of a new mixture rule or from measurements of light scattering. Physical Review, 68:5-10, 1945.

[22] J.V. Jelley. Chereknov Radiation and its Applications. Pergamon Press, 1958.

[23] A. Lebedev. Choosing the best momentum for $K^{+}$mass measurement. MIPP Note 97, 2005. 
[24] A. Lebedev. MIPP momentum scale based on RICH rings. MIPP Note $53,2005$.

[25] A. Lebedev. Chamber tuning and alignment. MIPP Note 129, 2007.

[26] Andre Lebedev, Holger Meyer, and Rajendran Raja. Progress in understanding the causes of the RICH fire. http://ppd.fnal.gov/ experiments/e907/RICH/accident/rich_fire_causes.pdf, April 2004.

[27] Andrey V. Lebedev. Ratio of Pion Kaon Production in Proton Carbon Interactions. PhD thesis, Harvard University, Cambridge, MA, May 2007.

[28] Valeri Lebedev. http:://fnal.gov/optim.

[29] W.R. Leo. Techniques For Nuclear and Particle Physics Experiments. Springer-Verlag, second edition, 1994.

[30] J.B. Lindsay et al. A fast and flexible data acquisition system for multiwire proportional chambers and other detectors. Nucl. Instr. and Meth., 156:329-333, 1978.

[31] J. Litt and L. Meunier. Cherenkov counter technique in high energy physics. Annu. Rev. Nucl. Sci., 23:1-44, 1973.

[32] G.K. Lum et al. Kaonic mass by critical absorption of kaonic-atom $\mathrm{x}$ rays. Phys. Rev. D, 23:2522, 1981. 
[33] James Priest. Russian and Hamamatsu Burn Tests. http://ppd.fnal. gov/experiments/e907/RICH/accident/MIPPbasetestBurnr1.pdf, April 2004.

[34] Rajendran Raja. Measuring the opening temperature of the two surviving Klixons. http://ppd.fnal.gov/experiments/e907/RICH/accident/ klixons.pdf, March 2004.

[35] J. Seguinot and T. Ypsilantis. Photo-ionization and Cherenkov ring imaging. Nucl. Instr. and Meth., 142:377-391, 1977.

[36] L. Stutte, J. Engelfried, and J. Kilmer. A method to evaluate mirrors for Cherenkov counters. Nucl. Instr. and Meth. A, 369:69, 1996.

[37] J. Thompson, D.E. Karus, and A. Sher. New, high statistics measurement of the $K^{+} \rightarrow \pi^{0} e^{+} \nu\left(K_{e 3}^{+}\right)$branching ratio. In Workshop on the CKM Unitarity Triangle, 2003.

[38] T. Ypsilantis and J. Seguinot. Theory of ring imaging Chereknov counters. Nucl. Instr. and Meth. A, 343:30-51, 1994.

[39] V.P. Zrelov. Chereknov Radiation in High-Energy Physics. Israel Program for Scientific Translations, Ltd., 1970. 


\section{Nicholas J. Graf}

727 E. Third St.

Bloomington, IN 47405

812-855-5705

ngraf@indiana.edu
800 N. Smith Rd.

Bloomington, IN 47408

812-336-7823

\section{Education}

\section{Indiana University}

Ph.D. Candidate, High Energy Physics, degree expected August 2008. Advisor: Dr. Mark Messier.

Thesis: "Measurement of the Charged Kaon Mass with the MIPP RICH".

\section{Rose-Hulman Institute of Technology}

B.S., Physics, May 2000.

\section{Research Experience}

\section{Indiana University}

Research Assisstant, MIPP Experiment, Dr. Mark Messier, 2003-Present. Refurbish, assemble and test Ring Imaging Cherenkov detector. Develop Slow Controls software using Python, MySQL database. Develop RICH reconstruction software in $\mathrm{C}++$. Participate in data taking. Analyze RICH data for charged kaon mass measurement.

Summer Research Assistant, Task D, Dr. Richard Heinz, 2001-2002.

Tested scintiallator bars, built and tested prototype light guide for Time-of-Flight system. 
Nicholas J. Graf, page 2

\section{Teaching Experience}

\section{Indiana University, Physics Department}

Laboratory Instructor, Physics 201: Fall 2000, Fall 2001, Spring 2002.

Laboratory Instructor, Physics 202: Spring 2001.

Laboratory Instructor, Physics 221: Fall 2001, Fall 2002.

Laboratory Instructor, Physics 222: Spring 2003.

Recitation Leader, Physics 201: Fall 2000, Spring 2002.

\section{Publications and Presentations}

T.S. Nigmanov et al. Electromagnetic and hadronic calorimeters in the MIPP Experiment. Submitted for publication, Nucl. Instr. and Meth. A, 2008.

R.J. Abrams et al. The Fermilab MIPP Experiment (FNAL-E907). Future submission, Nucl. Instr. and Meth. A.

"Possibility of charged kaon mass measurement using the MIPP RICH", Sixth International Workshop on Ring Imaging Cherenkov Detectors, Trieste, Italy, October 2007. 